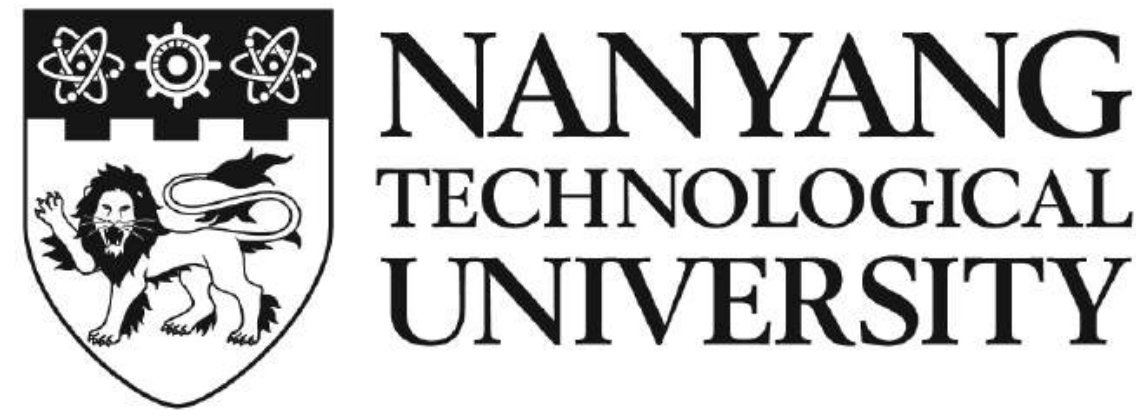

SPATIO-TEMPORAL MODELLING OF POPULATION AND URBAN MOBILITY

\author{
CHERYL ABUNDO
}

SCHOOL OF PHYSICAL AND MATHEMATICAL SCIENCES 2017 


\title{
SPATIO-TEMPORAL MODELLING OF POPULATION AND URBAN MOBILITY
}

\author{
by \\ CHERYL ABUNDO \\ Dissertation Supervisor: Assoc. Prof. Lock Yue Chew
}

A dissertation submitted to the

School of Physical and Mathematical Sciences

in partial fulfilment of the

requirements for the degree of

Doctor of Philosophy

School of Physical and Mathematical Sciences

Nanyang Technological University

July 2017 


\section{Acknowledgements}

Taking on a doctorate degree requires not only intellectual but emotional hard work. Behind every $\mathrm{PhD}$ student is a support group to laugh with, cry with, celebrate with, rant with, or simply as a reminer that there is more to life other than research. This thesis wouldnt have taken its form and would have just remained an idea if not for all those (both animate and inanimate, supernatural and natural, divine and mundane) Ive interacted with the past years. Your imprints are all over this thesis.

To the Nonlinear Dynamics and Complex Systems Lab, then and now, you have been dependable companions in this journey. To Khi Pin, Hendrik, Chong, Wei Liang, Nguyen and Andri who have been there in most of my PhD life, thank you for making every research group meeting, conference, talk and moments in the lab not just bearable but enjoyable.

To my Singapore friends, thank you for being my extended family here. Manu, Erin, Ara, Miguel and Raph, you've made Singapore less lonely and more fun.

To my virtual overseas friends who have always made themselves available in WhatsApp, Messenger, Facebook, Hangouts, Skype or Telegram, there were a lot of battles I had to fight during the course of my PhD and without you I can't imagine winning them. Mafie, James, Arianne, Lianne, Ruth, Alva and Miguel, you have been the best overseas friends I can ask for. Your constant presence (in my phone mostly) and willingness to listen helped me fight my wars within and have kept me sane throughout this process.

To my collaborators, Ning Ning, Guy, Murray, Elsa, Sean and Stefan, I've learned a lot and grown as a researcher from having been able to work with you. This thesis is a result of discussions and inputs from you. It has been a joy working with you and I hope this would not be the last.

To my supervisor Lock Yue and my research mentor Steve, thank you for guiding me and being patient with me. You shaped me into the researcher and person I am now. I am glad that you were my mentors. You were not only there to help me with my research but you were also there to understand and support me during the struggles I had these past years.

To Sir Chris and Erika, I wouldn't be here in Singapore doing my $\mathrm{PhD}$ if not for you. From UP days up to now, you have been my research mentors, life coaches 
and friends. You are one of the few who have always believed in me and have tolerated and accepted all my eccentricities. Hindi naging madali ang buhay $\mathrm{PhD}$ dito sa Singapore, salamat na lamang at lagi kayong nandiyan para tulungan ako sa research man o sa ibang aspeto ng buhay. I thank all the forces in the universe for conspiring to make me your advisee. Sa akin ang karangalan at kasiyahan.

To my family, Mommy, Ate Bianca, Ate Rea, Kuya Gel, Adi and Kyle, your unconditional love and support is felt wherever I am. It is always reassuring to know that I have a home to go back to whenever life feels tough.

Daddy, I miss you. Wherever you are, I know you are the most proud. I love you Daddy!

Alfonso, it's not easy being the boyfie of a PhD student. Thank you for loving me and caring for me even when it was difficult. All that I have achieved is yours as well. Thank you for reminding me to slow down and calm my mind and just enjoy the journey. You are my home. Te quiero mucho! 


\section{Contents}

Acknowledgements $\quad$ ii

List of Tables $\quad$ v

List of Figures $\quad$ vi

Abstract vii

1 Introduction $\quad 1$

1.1 Population Mobility . . . . . . . . . . . . . . 3

1.2 Urban Mobility . . . . . . . . . . . . . . . . . 6

1.3 Aims of the study . . . . . . . . . . . . . . . 9

2 Genetic Diversity 11

2.1 Genetic Tree . . . . . . . . . . . . . . . . . 14

2.1.1 mtDNA tree and distances . . . . . . . . . . . . . 14

2.1.2 Y chromosome STR Tree . . . . . . . . . . . . . . . 16

2.1.3 Molecular Dating of The Tree . . . . . . . . . . . . . 16

2.2 Two-deme Model of Migration in a Structured Population . . . . . . 16

2.2.1 Diversity comparison of uniparentally inherited genes . . . . . 18

2.2.2 Two-deme simulation of kinship-structured migrations . . . . 22

2.3 n-deme Model of Migration in a Structured Population . . . . . . . . 24

2.4 Genetic Diversity in Sumba and Timor . . . . . . . . . . . . . . . . 26

2.5 Adherence to Kinship Practice . . . . . . . . . . . . . . . . . . . . . . 28

2.6 Summary and Conclusions . . . . . . . . . . . . . . . . . . 30

2.7 Future Work . . . . . . . . . . . . . . . . . . . . . . 31 
3 Kinship Channels of Language and Genes

3.1 Association between genes and languages . . . . . . . . . . . . 33

3.1.1 Methods of shared gene-language heritage . . . . . . . . . . 34

3.1.1.1 Language Tree . . . . . . . . . . . . . . 34

3.1.1.2 Probability of shared gene-language heritage . . . . . 34

3.1.2 Results of shared gene-language heritage . . . . . . . . . . 34

3.2 Kinship and population genetic structure . . . . . . . . . . . . 38

3.2.1 Kinship Isolation with Migration (IM) model . . . . . . . . . 38

3.2.1.1 Fitting the kinship IM model using Approximate Bayesian Computation (ABC) . . . . . . . . . 40

3.2.1.2 Parameter space of the kinship IM model within and between villages . . . . . . . . . . . . . . . 42

3.2.2 Results of kinship and population genetic structure . . . . . . 42

3.2.3 Alternative isolation with migration model . . . . . . . . . 45

3.3 Kinship and language transmission . . . . . . . . . . . . 50

3.4 Co-phylogenetic association of languages and genes . . . . . . . . 53

3.4.1 Statistical test for gene-language coevolution . . . . . . . . 55

3.4.1.1 ParaFit statistic . . . . . . . . . . . . 55

3.4.1.2 Permutation Model . . . . . . . . . . . . 56

3.4.2 Results of co-phylogenetic association of languages and genes . 57

3.5 Summary and Conclusions . . . . . . . . . . . . . . . . . . 58

3.6 Future Work . . . . . . . . . . . . . . . . . . . . . 59

4 Coupling of Language and Gene Trees $\quad 61$

4.1 Co-evolution of languages and genes . . . . . . . . . . . . . 62

4.2 Cophylogeny reconstruction of language and gene trees . . . . . . . 64

4.2.1 Jane method of co-phylogeny reconstruction . . . . . . . . . . 64

4.2.2 Co-phylogeny of languages and genes . . . . . . . . . . . 64

4.3 Host switching and persistence of speech communities . . . . . . . . 66

4.4 Language switching between genetic clades . . . . . . . . . . . . . 69

4.4.1 Language switching on genetic trees . . . . . . . . . . . 70

4.4.2 Alternative model of language switching between genetic clades 73

4.5 Summary and Conclusions . . . . . . . . . . . . . . . 74

4.6 Future Work . . . . . . . . . . . . . . . . . . . . 77 
5 Mobility in Transport Networks $\quad 78$

5.1 Conventional models of human mobility . . . . . . . . . . . . . . . 80

5.1 .1 Gravity model . . . . . . . . . . . . . . . 80

5.1 .2 Intervening opportunities model . . . . . . . . . . . . . . 81

5.1 .3 Original radiation model . . . . . . . . . . . . . . . 82

5.2 Radiation model modified for urban travel . . . . . . . . . . . . . . . 82

5.2.1 Rapid Transit System . . . . . . . . . . . . . . 82

5.2.2 Characteristic parameters of the radiation model modified for urban travel . . . . . . . . . . . . . . . . . . 83

5.2.2.1 Urban land-use . . . . . . . . . . . . . . . 84

5.2.2.2 Opportunities density . . . . . . . . . . . 84

5.2.2.3 Boundary porosity . . . . . . . . . . . . 87

5.2.2.4 Transport network structure . . . . . . . . . . 88

5.2.3 Enhanced radiation model . . . . . . . . . . . . . . . 90

5.3 Comparison of results from the modified radiation model with realdata from Singapore RTS . . . . . . . . . . . . . . . . . 93

5.4 Summary and Conclusions . . . . . . . . . . . . . . . . . . . 99

5.5 Future Work . . . . . . . . . . . . . . . . . . . . . . 100

6 Fragmentation and Resilience of Networks 102

6.1 Network resilience . . . . . . . . . . . . . . . . . . . . . . . 103

6.1.1 Measures of network resilience . . . . . . . . . . . . . . 104

6.1 .2 Models of network resilience . . . . . . . . . . . . . . 106

6.1.2.1 Network structure . . . . . . . . . . . 106

6.1.2.2 Network dynamics . . . . . . . . . . . . . . 108

6.1.2.3 Failure mechanism . . . . . . . . . . . . . 108

6.1.2.4 Spring-mass model of network fragmentation . . . . 109

6.2 Spatial grid network . . . . . . . . . . . . . . . . . . 110

6.2.1 Force accumulation . . . . . . . . . . . . . . . . . 114

6.2.2 Breaking dynamics . . . . . . . . . . . . . . . . . 114

6.3 Lattice network . . . . . . . . . . . . . . . . . . . 117

6.3.1 Uniform force directions . . . . . . . . . . . . . . . . 117

6.3.2 Random force directions . . . . . . . . . . . . . . . . 120

6.4 Erdos-Renyi network . . . . . . . . . . . . . . . . . . . . . . . 121 
6.4.1 Uniform force directions . . . . . . . . . . . . . . . . . . 121

6.4.2 Random force directions . . . . . . . . . . . . . . . . . . 122

6.5 Barabasi-Albert network . . . . . . . . . . . . . . . . . . 122

6.5.1 Uniform force directions . . . . . . . . . . . . . . . . 122

6.5.2 Random force directions . . . . . . . . . . . . . . . . 127

6.6 Dependence of the preservation of links on the randomness of the force directions . . . . . . . . . . . . . . . . . . . . . . 127

6.7 Lifetime of grid network . . . . . . . . . . . . . . . . . 129

6.8 Application to a real-world transport network . . . . . . . . . . . . . 132

6.9 Summary and Conclusions . . . . . . . . . . . . . . . . . . . . 138

6.10 Future Work . . . . . . . . . . . . . . . . . . . . . . . 141

7 Conclusions and Future Work $\quad 143$

$\begin{array}{lr}\text { Bibliography } & 148\end{array}$

A Appendix 162

A.1 Network Elongation . . . . . . . . . . . . . . . . . . . 162

A.2 Numerical Stability . . . . . . . . . . . . . . . . . 163

A.3 Distribution of Influence . . . . . . . . . . . . . . . . . 163

A.3.1 Lattice Network . . . . . . . . . . . . . . . . . . . 163

A.3.1.1 Uniform Force Directions . . . . . . . . . . . . 163

A.3.1.2 Random Force Directions . . . . . . . . . . . . . . . 164

A.3.2 Erdos-Renyi Network . . . . . . . . . . . . . . . 165

A.3.2.1 Uniform Force Directions . . . . . . . . . . . . 165

A.3.2.2 Random Force Directions . . . . . . . . . . . . . 165

A.3.3 Barabasi-Albert Network . . . . . . . . . . . . . . . 166

A.3.3.1 Uniform Force Directions . . . . . . . . . . . . 166

A.3.3.2 Random Force Directions . . . . . . . . . . . 166

A.4 Barabasi-Albert Network Configurations . . . . . . . . . . . . . 167

A.5 Generalization to Other Kinds of Spatial Networks . . . . . . . . 168

A.6 Cluster Sizes . . . . . . . . . . . . . . . . . . . . . . . 169 


\section{List of Tables}

2.1 Kinship and post-marital residence rules. Culturally prescribed rules of residence post marriage is described below. . . . . . . . . . . . 13

3.1 Z scores of gene-language associations. Comparing within each group of villages (columns), stronger gene-language associations (shading) are found for $\mathrm{Y}$ in all Sumba villages, mtDNA in all Timor villages, mtDNA in matrilocal Timor villages, and $\mathrm{Y}$ in patrilocal Timor villages. 57

4.1 Host switching and loss rates per generation. Rates are computed for the relatively recent past of 10 kya to present. Cells in gray are the cases that also shows strong association of languages and genes. . . . 68 


\section{List of Figures}

1.1 Spatial and temporal scales. Mobility is modelled for (1) population migrations (bottom red) that fall under a spatial scale of $\sim 10^{6} \mathrm{~m}$ and long time scales of years to centuries, and (2) urban travels (top green) involving regions of $\sim 10^{3}-10^{4} \mathrm{~m}$ and short time scales of seconds to days. . . . . . . . . . . . . . . . . .

1.2 Genetic haplogroups in sampled villages of Sumba and Timor. Diversity of (Top) mtDNA haplogroups and (Bottom) Y chromosome STR haplogroups of villages in (Left) Sumba and (Right) Timor is shown with - - indicating the patrilocal villages and • indicating the matrilocal villages. Pie charts scaled by sample size show the distribution of haplogroups in the villages. . . . . . . . . . . . . . 4

1.3 Languages in sampled villages on Sumba and Timor, eastern Indonesia. Pie charts show the languages spoken, scaled by sample size. Left: 14 patrilocal villages (-) on Sumba and the Austronesian languages spoken by the 505 sampled men. Right: 11 communities on Timor, including 9 matrilocal villages $(\bullet)$ and 2 patrilocal villages (•). Each of the 477 men sampled on Timor speaks one or more of five local languages belonging to two language families, Papuan (Bunak) and Austronesian (Betun, Dawanta, Kemak and Upper Tetun). Multiple languages are typically spoken within communities in Timor, while only one language is typically spoken within a given community in Sumba. . . . . . . . . . . . . . . . . .

1.4 Urban mobility network layers. Mobility events in urban areas are defined by the coupling of land-use entities in the urban planning map, gross plot ratio proxy of population density and transport network attributes. . . . . . . . . . . . . . . . . 7 
1.5 Travel demand forces. A stochastic fatigue model is used to follow the evolution of the Singapore bus network as travel demand forces cause strain along its network links. Bigger and lighter nodes are the often used bus stops. Links are thicker if demand for the routes is higher. . . . . . . . . . . . . . . . .

2.1 mtDNA genetic tree. The female lineage of the sampled Indonesian men from (A) Sumba and (B) Timor colored according to their mtDNA haplogroup. . . . . . . . . . . . . . . .

$2.2 \mathrm{Y}$ chromosome STR genetic tree. The male lineage of the sampled Indonesian men from (A) Sumba and (B) Timor colored according to their Y chromosome STR haplotype. . . . . . . . . . . . . . . . 1

2.3 Movement assumptions for agent-based model of kinship-driven migrations between structured populations. Schematic diagram of the rules followed by every individual that moves per time step in the agent-based model is shown. For every pair of interacting demes (shown as circles), every individual that move (represented by the arrows) is replaced by a new individual randomly chosen from the subpopulation's existing pool. A - in the table below the circles indicate the subtraction of one male/female and $\mathrm{a}+$ is an addition of one male/female in the deme (circle 1 or circle 2). (A) and (B) shows that a male/female moving out of sub-population 1 (indicated by -leaving ) is replaced by a male/female bred from a randomly chosen male and female in sub-population 1 (indicated by +inbred ). A randomly chosen male/female in sub-population 2 (indicated by -dying ) is replaced by the immigrating male/female. The immigrant and his randomly chosen mate (indicated by - mate) is then replaced by their son (indicated by + son ) and daughter (indicated by + daughter $) \ldots \ldots \ldots \ldots \ldots$. . . . . . . . . . 
2.4 Two-deme kinship-structured migration interaction. The top row shows the replacement of mtDNA $m t_{i}$ and $\mathrm{Y}$ chromosome $y_{i}$ after one migration event when both demes have the same kinship practice. (A) Patrilocal-patrilocal interaction preserves $y_{i}$ deme homozygosity while (B) matrilocal-matrilocal interaction preserves $m t_{i}$ deme homozygosity. (C) Neolocal-neolocal can stochastically show either a "patrilocal" or a "matrilocal" signal. (D) Endogamous interactions mantain the original $m t_{i}$ and $y_{i}$ compositions of the demes. The second row shows bidirectional interactions of villages heterogeneous in kinship practice. (E) Patrilocal-matrilocal interaction causes villages to show a signal opposite to its actual kinship practice. (F) Patrilocal-neolocal and $(\mathrm{G})$ matrilocal-neolocal interactions results to the neolocal village adopting the signal of the other village which in turn cross-dresses. The bottom row of unidirectional migration shows $(\mathrm{H})$ patrilocal-endogamy, (I) matrilocal-endogamy and $(\mathrm{J})$ neolocalendogamy interactions. In $(\mathrm{H})-(\mathrm{J})$, non-endogamous villages show no change in genetic composition while endogamous villages give the signal of the village it interacts with. White villages emits a signal similar of its actual kinship practice, orange villages are cross-dressers, yellow villages are neolocal villages that can either be "matrilocal" or "patrilocal" while violet villages maintain their original genetic composition. . . . . . . . . . . . . . . . 21

2.5 Two-deme simulation of kinship-structured migrations. (A) Patrilocalpatrilocal, (B) matrilocal-matrilocal and (C) neolocal-neolocal migrations show the expected $p_{m m}-p_{y y}$ signal. When the exchange of males or females is assymetric as in (D) patrilocal-matrilocal, (E) patrilocalneolocal, (F) matrilocal-neolocal, $(\mathrm{G})$ patrilocal-endogamy and $(\mathrm{H})$ matrilocal-endogamy, cross-dressing is observed. (I) Neolocal-endogamy interactions results to one homogeneous and one well mixed villages. The trends appear distinct by around generation 200 and are sustained in all generations. . . . . . . . . . . . . . . 23 
2.6 n-deme simulation of kinship-structured migrations. Comparison of mtDNA and Y chromosome similarities for a population divided into 10 demes by kinship-structured interactions: (A) all patrilocal, (B) all matrilocal, (C) 5 patrilocal and 5 matrilocal, (D) 8 patrilocal and 2 matrilocal, (E) 2 patrilocal and 8 matrilocal, and (F) 3 patrilocal, 3 matrilocal, 2 neolocal and 2 endogamous villages. The networks on the top left of the plots are the migration networks of males while those on their top right are the migration networks of females. Every link corresponds to an $m=1.0$. The mean $p_{m m}-p_{y y}$ is presented as solid lines together with the standard deviation of $2^{4}$ realizations. Cross-dressing is observed in $(\mathrm{C})-(\mathrm{F})$ with the neolocal villages in $(\mathrm{F})$ switching between being "patrilocal" and "matrilocal" in the course of the simulations. . . . . . . . . . . . . . . . 25

$2.7 m t D N A$ and $Y$ chromosome diversity comparison in Sumba and Timor. $p_{m m}-p_{y y}$ is shown in the top row for the 12 matrilocal villages of Sumba and in the bottom row for the 2 patrilocal villages and 9 matrilocal villages of Timor. (A) Sharing of a common ancestor in the mtDNA and Y chromosome trees of all individuals in Sumba show "matrilocality" signal except for time depths 51 - 57 kya. (B) Analyzing the villages separately show transient cross-dressings. (C) The probability comparison of sharing an ancestor in the mtDNA and Y chromosome trees of all, matrilocal and patrilocal individuals of Timor is shown. Patrilocal villages always cross-dress while matrilocal villages switch locality signal. (D) Timor patrilocal villages cross-dresses until 58 kya while matrilocal villages behave like neolocal villages with some showing "patrilocality" and some "matrilocality". 27 
2.8 Effects of relaxed kinship practice and limited interactions to joint genetic diversity (A) Matrilocal Sumbanese villages under strict kinship practice shows similar mtDNA and Y chromosome diversity due to its net female village populations. (B) Dissimilar genetic diversities and cross-dressing for the villages initialized to be Sumbanese happens when kinship practice is relaxed and dispersals are limited. (C) For Timorese villages, only patrilocal villages cross-dress under strict kinship practice. (D) Relaxing rules and limiting interactions result to transient cross-dressings for all Timorese villages. . . . . . . . . .

3.1 Language Tree. The root of the (A) Sumba language tree is set at 4.085 kya. In (B) Timor, the Papuan language Bunak splits off at 10 kya and the Timorese Austronesian languages at 5 kya. These calibration points are shown as $\bullet . \ldots \ldots . \ldots . \ldots 35$

3.2 Language sharing in the mtDNA and Y phylogenies of (A-B) Sumba and (C-D) Timor. Color bands beneath the phylogenies show the languages spoken by each individual (monolingual in Sumba; sometimes multilingual in Timor). Plots to the right of each phylogeny show the probability of sharing a language $l$ given that each pair of individuals are in the same genetic clade $g$ at a given time in the past. Solid lines represent the observed metric, with shaded bands indicating the results of random permutations of the linguistic data. Higher probabilities that close genetic relatives share a language, compared with random expectations, are observed for (B) Sumba Y and (C) Timor mtDNA at all time periods. For (A) Sumba mtDNA and (D) Timor $\mathrm{Y}$, probabilities are only higher than random cases until $\sim 65,000$ and $\sim 1,500$ years ago, respectively. . . . . . . . . . . . 36

3.3 Posterior distributions of IM model fitting for Sumba data, based on best-fitting 150 parameter values of approximately 2 bn samples from prior distributions as indicated. . . . . . . . . . . . . . . . . 41

3.4 Posterior distributions of IM model fitting for Timor data, based on best-fitting 150 parameter values of approximately 2 bn samples from prior distributions as indicated. . . . . . . . . . . . . . . . . . . 44 4 
3.5 Migration rates phase space. (A) KL divergence of pairwise distance distributions $(\mathrm{KL}(\mathrm{Y}-\mathrm{mtDNA})+\mathrm{KL}(\mathrm{mtDNA}-\mathrm{Y}))$ within subpopulations. (B) KL divergence of pairwise distance distributions between subpopulations. (C) Example plots of mtDNA (blue lines) and Y chromosome (red lines) pairwise distance distributions within (solid line) and between (dashed line) subpopulations, focusing on the lowdifference regime. . . . . . . . . . . . . . . . . 43

3.6 Genetic structure from an IM model with migration influenced by kinship practices. To identify the theoretical role of kinship practices on genetic diversity, the IM model was run for a population that are (A) endogamous, (B) ambi- or neolocal, (C) patrilocal and (D) matrilocal. (E) Close correspondence of the IM model (red shading) with observed data (blue contours) for patrilocal Sumba using $N=$ $298, n=41, m_{\text {female }}=0.73, m_{\text {male }}=9 x 10^{-5}, \tau=5.49$ and $a=0.024$. (F) Close correspondence of the IM model with matrilocal Timor using $N=264, n=97, m_{\text {female }}=1 x 10^{-5}, m_{\text {male }}=0.25, \tau=6.71$ and $a=0.06$. The insets (E) and (F) show the posterior distributions of migration rates for the kinship system in Sumba and Timor based on 3 billion samples drawn from prior distributions of all IM model parameters. ....................... . . . 44

3.7 Two-stage isolation with migration model. First isolation with migration occurs between two sub-populations at time $T_{0}$. Sub-population 1 with size $2 N$ further divides into $n$ sub-populations at time $T_{1} \ldots$. . 46

3.8 Genetic distance depending on kinship practice. The two-stage genetic distance isolation with migration model was run for a population with villages that are (A) endogamous, (B) neolocal, (C) patrilocal and (D) matrilocal. . . . . . . . . . . . . . . . . . . . 49 
3.9 Genetic distances on Sumba (left, A\&C) and Timor (right, B\&D), both between all pairs of individuals (A-B) and only between individuals who speak a language in common (C-D). Conditioning on language sharing reveals three distinct clusters in the Sumba data (C), showing evidence of village endogamy (e), ambi- or neolocality (an) and patrilocality (p). For Timor, the high degree of multilinguality means that most pairs of individuals in B are also included in D. B and D show matrilocality (m) and ambi- or neolocality (an),

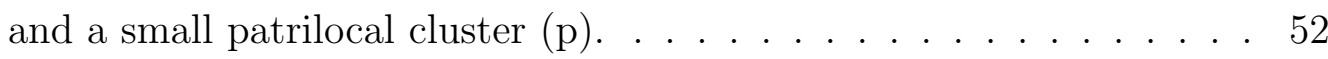

3.10 Linguistic and genetic distances. The kernel density of the linguistic and genetic distances of all pairs of individuals is shown. (A) In Sumba where villages are monolingual, only a few pairs with mtDNA distance $\sim 8$ speak the same language. (B) Looking at the Y chromosome in Sumba, pairs of individuals speaking the same language form two clusters of close $(\sim 0.0)$ and slightly distant $(\sim 5-10)$ relatedness.(C) In Timor, there is a high probability of finding pairs of individuals that are both closely related in their mtDNA and in the languages they speak. (D) Most pairs of individuals in Timor have a distant degree of relatedness in their Y chromosome but speak similar set of languages. . . . . . . . . . . . . . . . . . . . 54

3.11 Fourth-corner matrix. Statistics describing gene-language associations. 56

3.12 Permutation model. Columns of the $L G$ matrix are shuffled to remove associations, while preserving the number of multilingual individuals and the number of languages they speak. . . . . . . . . . 5 56

4.1 Languages and genes cophylogeny correlation. (A) Strong correlation of languages and genes exists as a result of only cospeciation events. (B) Only some correlation remains if in between cospeciation events, duplication, host switching or loss happens. (C) Almost all correlation is lost if frequent host switching and loss occurs. . . . . . . . . 63 
4.2 Languages in the (A) matriline (mtDNA) and $(B)$ patriline ( $Y$ chromosome) phylogenies. The first color bands in (A) and (B) are all the languages spoken by every individual in the phylogenies. Multilinguality is represented by multiple colors in the first color bands below the individuals. The second color bands visually represent the size of speech communities. The largest speech community in (A) is bigger (69 individuals) with an older most recent common ancestor $(\sim 55$ kya) as compared to the largest speech community in (B) with 26 individuals genetically belonging to two sub-clusters with most recent common ancestors at $\sim 29$ kya and $\sim 20$ kya. . . . . . . . . . 65

4.3 Cophylogenetic events. There are five plausible events that languages can experience as it evolve with genes: (1) codivergence, (2) loss, (3) duplication, (4) host switch and (5) failure to diverge). . . . . . . . 66

4.4 Language and gene trees co-phylogeny. The co-phylogeny reconstruction from Jane of significant associations is shown for (A) mtDNA matrilocal villages, (B) mtDNA patrilocal villages, (C) Y chromosome matrilocal villages and (D) Y chromosome patrilocal villages.

4.5 Maximum size of speech community. Matrilocal villages are more likely to have a bigger speech community in their mtDNA while patrilocal villages are more likely to have it bigger in their Y chromosome. 67

4.6 p-value for the different host switching rates. Host switching must occur below $5 \%$ for the p-value of language-gene association to be significant. . . . . . . . . . . . . . . . . . . 68

4.7 Language switching in a gene tree conditioned on a language tree. Shown in (A) is an unannotated gene tree, (B) a language tree and (C) an example of a simulated language-annotated gene tree. Language diversifies during language branching ( $L_{123}^{*}$ splits into $L_{12}^{*}$ and $L_{3}$ at $t_{2}$ ), and during host switching, as in the branches leading to $G_{2}$ (language switch at $t_{4}$ ) and $G_{3}$ (language switch at $t_{5}$ ). . . . . 70 
4.8 Language switching rates. The $Z$ score measure of association between gene and language phylogenies on Sumba and Timor for different language switching rates $\alpha$. All cases independently converge and abruptly lose gene-language associations, behaving similar to randomized cases, when the language switch rate exceeds $\sim 0.5 \%$. . . . . 72

4.9 Language switching rates from the alternative model.The $Z$ score measure of association between gene and language phylogenies on Sumba and Timor for different language switching rates $\alpha$ in the alternative model. All cases independently converge and abruptly lose gene-language associations, behaving similar to randomized cases, when $\alpha \approx 5 \%$. . . . . . . . . . . . . . . . . . 74

5.1 Singapore Mass Rapid Transit. 2008 urban planning map of Singapore with four MRT lines (Red - North South Line, Green - East West Line, Purple - North East Line, Yellow - Circle Line) having a total of 90 stations and 97 links. . . . . . . . . . . . . 83

5.2 Land use entities. The defining mobility features in Singapore Rapid Transit System (RTS). (a) Business, (b) commercial, (c) commercial and residential, (d) hotel, (e) health and medical care, and (f) educational institution are considered "work" entities while (g) residential entities are considered "home" locations. . . . . . . . . . . . . 85

5.3 Gross plot ratio for the land use entities. Points refer to the locations in the map for which a GPR measure is available for each of the land use entities considered. . . . . . . . . . . . . . . . . . . 86

5.4 Singapore MRT voronoi regions of influence. Each station serves a region of commuters as indicated above. . . . . . . . . . . . . 88

5.5 Singapore RTS network weighted by density estimates for each land use entities. Network node sizes are weighted according to normalized sum of GPR values for each of the considered land use types. Network is superimposed over the Voronoi tesselation. . . . . . . . . . . . . 89

5.6 Correlation of RTS network node centrality measures with data. The bigger and darker the network nodes are, the higher the node centrality measures. The unfilled blue circles are mean of total tapins in the stations per day over a five weekday period. . . . . . . . . . . . . 91 
5.7 Modified Radiation Model. A commuter in region $S_{i}$ with absorption threshold dependent on potential $g_{i}^{O}$ decides to travel to region $S_{j}$ with absorbance dependent on potential $g_{j}^{D}$ given the intervening absorbances dependent on potential $s_{i j}^{D}$ in region $R_{v_{i}}$. . . . . . . . 92

5.8 Travel demand prediction. Predicted values have correlation of 0.70 with data. Filled circles in (a) represent predicted values while unfilled blue circles are from the data. . . . . . . . . . . . . . . . . . . . 94

5.9 Travel demand prediction for morning and afternoon rides. Prediction for morning trips has a correlation of 0.74 with the data while that for afternoon trips has 0.71 . The blue regions represent the predicted mean and standard deviation of travel demand. . . . . . . . . 95

5.10 Travel demand prediction for MRT lines. Correlation of predicted values with data for the (a) Circle, (b) East West, (c) North East and $($ d) North South lines. . . . . . . . . . . . . . . . . 96

5.11 Standard deviations of station travel demand. The travel demand standard deviations of the model and the data are shown in (a) with the blue line being the best fit line. (b) shows the probability distributions of the standard deviations of the model and data. The data has higher variances than model predictions. . . . . . . . . . . . 96

5.12 Proportion of actual travel demand fluctuations captured by model. The proportion of the standard deviations of the model and the data, $\sigma_{\text {model }} / \sigma_{d a t a}$, for the mean data travel demand $\mu_{\text {data }}$ is shown in (a). (b) shows the histogram of $\sigma_{\text {model }} / \sigma_{\text {data }}$. The stations within the red region represent those for which $\sigma_{\text {model }}$ is within $50 \%$ of $\sigma_{\text {data }} \ldots \ldots 97$

5.13 Difference in fluctuations of model and data. The size of the nodes corresponds to $\mu_{\text {data }}$. Nodes are colored according to $\sigma_{\text {data }}-\sigma_{\text {model }} . \quad$. 98

6.1 Percolation. (a) Below the critical probability, $p_{c}$, the network is composed of isolated clusters. Above $p_{c}$, a giant cluster spans the network. (b) The average cluster size, $\langle s\rangle$ diverges at $p_{c}$. (c) A giant cluster appears at $p_{c}$ and grows until the network is fully connected. . . . . . . . . . . . . . . . 105 
6.2 Error and attack tolerance. The relative size of the giant cluster $S$ (open symbols) and the average size of isolated clusters $\langle s\rangle$ (filled symbols) as a function of the fraction of removed nodes $f$ are shown. (a) Fragmentation of the exponential random network, E, under random failures (squares) and attacks (circles). (b) Fragmentation of the scale-free network, SF, under random failures (blue squares) and attacks (red circles). The inset in (b) shows the error tolerance curves for the whole range of $f$. Scale-free networks are more robust than random networks against random node failures but are more vulnerable against targeted attacks. (Figure from [1].) . . . . . . . . . . 107

6.3 Lattice network architecture and index conventions. (a) represents a lattice network with uniform force directions and (b) random force directions. Edge colors correspond to the elongation thresholds $\epsilon_{t h}$ while node colors are based on the degree of incoming connections $k_{i n}$. Node sizes correspond to the degree of outgoing connections $k_{\text {out }}$ (largest node has higher $k_{\text {out }}$ ). Arrows are directed from source to target node. . . . . . . . . . . . . . . . . . . 111

6.4 Erdos-Renyi network architecture and index conventions. In (a) and (b) nodes are arranged spatially in a grid and $[(M-1) \times N]+[M$ $\mathrm{x}(N-1)$ ] edges are randomly chosen from possible connections. (a) represents an ER network where forces are directed with the highest level of uniformity and (b) randomly. Edge colors correspond to the elongation thresholds $\epsilon_{t h}$ while node colors are based on the degree of incoming connections $k_{i n}$. Node sizes correspond to the degree of outgoing connections $k_{\text {out }}$ (largest node has higher $k_{\text {out }}$ ). Arrows are directed from source to target node. Figures in the last column highlight the edges of the shown selected nodes. . . . . . . . . . . . . 112

6.5 Barabasi-Albert network architecture and degree distribution with uniform and random force directions. Almost all edges were directed outward for the uniform case while comparable number of edges were directed inward and outward for the random case. In the inset, nodes are sized according to $k_{\text {out }} \ldots \ldots \ldots$. . . . . . . . . . 113 
6.6 Body diagram. Displacement of the $(i, j)^{\text {th }}$ node and its four nearest neighbors. . . . . . . . . . . . . . . . . . 116

6.7 Evolution of a lattice network with uniform force directions. For $F_{a p p}=0.03$, breaking starts near the top and left sides at $t=6,384$. Failure cascades and last to be disconnected are edges near the bottom right corner where the least amount of force is felt. The network totally collapses at $t=6,602.4375$. No connections remain and the network elongates and moves diagonally following the net force as shown by the red arrows in the network movement panel. . . . . . . . 118

6.8 Evolution of a lattice network with random force directions. For $F_{a p p}=0.03$, breaking starts at $t=11$ at random positions. The network reaches its equilibrium structure at $t=238.6$ with only $0.496 \pm 0.007$ of the edges disconnected. The top and right panels show the relative expansion of the network in space. . . . . . . . . . . 119

6.9 Evolution of an Erdos-Renyi network with uniform force directions. For $F_{a p p}=0.8$, breaking starts with nodes disconnecting from the influential nodes at the bottom resulting to $77.61 \%$ of the edges to break instantly. By $t=100,95.73 \%$ of the edges have disconnected. The remaining edges take far longer to disconnect. The top and right panels show the relative expansion of the network in space. . . . . . . 123

6.10 Evolution of an Erdos-Renyi network with random force directions. For $F_{a p p}=0.8$, breaking starts at random positions. Initial disconnections account for $79.6 \%$ of the edges. By $t=100,97.0 \%$ of edges have disconnected. The remaining $3.0 \%$ breaks slower until the network completely collapses at $t=7,273.1875$. The top and right panels show the relative expansion of the network in space. . . . . . . 124

6.11 Evolution of a Barabasi-Albert network with uniform force directions. For $F_{a p p}=0.8,75.9 \%$ of the edges fail instantly and by $t=128.0$, $96.7 \%$ of the edges has disconnected. The remaining edges have all disconnected by $t=28,692.8 \ldots \ldots \ldots$. . . . . . . . . . . . . . . . . . 
6.12 Evolution of a Barabasi-Albert network with random force directions. For $F_{a p p}=0.8,75.9 \%$ of the edges fail instantly with only $1.9 \%$ of the edges remaining by $t=128.0$. The network completely fragments at $t=22,773.6$

6.13 Fraction of disconnected edges $f$ in grid networks. (a) Lattice networks with uniform force directions totally collapse while for those with (b) random force directions, $0.503 \pm 0.006$ of the edges disconnects. (c-d) Regardless of the magnitude and directions of $\left\{F_{a p p}\right\}$, an ER network experiences total system collapse. (e-f) BA networks completely fragment regardless of the magnitude and directions of $\left\{F_{a p p}\right\}$. The insets show the derivative of $f$ or the disconnection rate of the edges. The rate of disconnection $f^{\prime}$ at the transition point decreases with $F_{a p p}$ in all networks. For lattice networks, randomizing force directions further decreased the rate of disconnections. . . . . . 128

6.14 The final fraction of disconnected edges in a lattice network with varying degree of force directions randomness. The relationship is found to be linear with a slope of -0.51. . . . . . . . . . . . . . . . 129

6.15 Lifetime of spatial grid networks. Dashed lines illustrate the Basquin's exponent of the networks. . . . . . . . . . . . . . . 130

6.16 Damage sequence waiting time. The macro-scale network lifetime results from microscopic scale-free waiting times between bursts. The lines shown are the range of exponents of the power-law distribution. 131

6.17 Degree distribution of Sinagpore bus network. . . . . . . . . . . . . . 133

6.18 Number of edges for each Fapp. Inset shows bus network edges weighted by $F_{a p p} \ldots \ldots \ldots$. . . . . . . . . . . . . . . . . . 134

6.19 Force directions in Singapore bus network. Force directions considered are (a) actual bus directions, (b) towards nodes with higher degree $k$, (c) away from nodes with higher degree $k$, and (d) random directions. Nodes are colored according to their in-degrees and their sizes are based on their out-degrees. Edges are weighted according to $F_{a p p}$. . . 135 
6.20 Singapore bus network evolution with actual bus routes as force directions. The network completely fragments at $t=31.125$. Nodes are colored according to in-degrees and their sizes are based on outdegrees. Edges are weighted according to $F_{a p p} \ldots \ldots$. . . . . . 136

6.21 Singapore bus network evolution with forces towards nodes with higher degree. The network completely fragments at $t=26.0625$. Nodes are colored according to in-degrees and their sizes are based on outdegrees. Edges are weighted according to $F_{a p p} \ldots \ldots$. . . . . . . 136

6.22 Singapore bus network evolution with forces away from nodes with higher degree. The network completely fragments at $t=37.4375$. Nodes are colored according to in-degrees and their sizes are based on out-degrees. Edges are weighted according to $F_{a p p} \ldots$. . . . . 137

6.23 Singapore bus network evolution with random force directions. The network completely fragments at $t=52.75$. Nodes are colored according to in-degrees and their sizes are based on out-degrees. Edges are weighted according to $F_{a p p} \ldots \ldots \ldots$. . . . . . . . . . . . . . .

A.1 Random reinforcement of network edges. (a) Elongation thresholds of the horizontal edges and (b) elongation thresholds of the vertical edges in the lattice network. . . . . . . . . . . . . . . . 162

A.2 Heatmap of a lattice network with uniform force directions. (a) The node in the topmost left corner is influenced by all other nodes and the degree of influence decreases diagonally towards the farthest reachable node at the bottom right corner. (b) Nodes are influenced only by nodes that are to their right and below them. (c) All forces are directed towards the most influential node in the bottom right corner. 164

A.3 Heatmap of a lattice network with random force directions. Influence is spatially distributed randomly. In (a) only $3.92 \%$ of the nodes has influence on the node in the topmost left corner of the network but in (b) we show a node having a greater influence range. (c) There are also more nodes $(6.24 \%)$ which are not influenced by any node. . . . . 165 
A.4 Heatmap of an Erdos-Renyi network with uniform force directions.

(a) Nodes can influence nodes that are not their spatial neighbors. The uniformity of force directions is illustrated in (b) where nodes can only be influenced by nodes below them. (c) The number of nodes that are not influenced by any node accounts for $6.24 \%$ of nodes in the network. . . . . . . . . . . . . . . . 166

A.5 Heatmap of an Erdos-Renyi network with random force directions. (a) Nodes are influenced by more nodes when both connections and force directions pattern are random. (b) This is true regardless of the position of the node in grid space. (c) There are also more nodes that are not influenced by any node which accounts for $14.12 \%$ of network nodes. . . . . . . . . . . . . . . . . . 167

A.6 Heatmap of a Barabasi-Albert network with uniform force directions.

(a) The node with the highest degree is connected to almost all nodes. As illustrated in (b), due to the uniformity of force directions and the scale-free degree distribution, other nodes are connected to very few nodes. (c) $49.28 \%$ of nodes in the network has no outgoing edge. . . . 168

A.7 Heatmap of a Barabasi-Albert network with random force directions.

(a) The node with the highest degree is connected to almost all nodes.

(b) Randomizing force directions allowed other nodes to have paths to more nodes. (c) $15.64 \%$ of nodes in the network has no outgoing edge. . . . . . . . . . . . . . . . . . 169

A.8 Barabasi-Albert network configurations. Hubs are either placed next to each other at the corner $(\mathrm{a}, \mathrm{c}, \mathrm{e})$ or in random positions $(\mathrm{b}, \mathrm{d}, \mathrm{f})$. Edges in both placements are directed away from the node with the higher degree $k(\mathrm{a}, \mathrm{b})$, towards the node with the higher degree $k(\mathrm{c}, \mathrm{d})$ or randomly $(\mathrm{e}, \mathrm{f}) \ldots \ldots \ldots \ldots$. . . . . . . . . . . . . . . . . . . . . . . .

A.9 Spatial Grid. For any spatial arrangement of nodes, a smallest spatial grid can be found such that all nodes lie in one and only one intersection in the grid. . . . . . . . . . . . . . . . . . . . 171

A.10 Number of clusters in the evolving lattice, Erdos-Renyi (ER) and Barabasi-Albert (BA) networks for $F_{a p p}=0.6$. A lattice with random forcing directions experiences the least amount of fragmentation. 172 
A.11 Cluster sizes of lattice networks. Size of the largest cluster $S$ and the average size of the smaller clusters $\langle s\rangle$ as the lattice network is subjected to (a) uniform and (b) random force directions with $F_{a p p}=$ 0.6. Random force directions leads to a percolating cluster while uniformity in force directions causes nodes to detach from each other. 172

A.12 Cluster sizes of Erdos-Renyi networks. Size of the largest cluster $S$ and the average size of the smaller clusters $<s>$ as the Erdos-Renyi network is subjected to (a) uniform and (b) random force directions with $F_{a p p}=0.6$. The network fragments early on in its evolution leading eventually to isolated nodes. . . . . . . . . . . . . . . . 173

A.13 Cluster sizes of Barabasi-Albert networks. Size of the largest cluster $S$ and the average size of the smaller clusters $\langle s>$ as the BarabasiAlbert network is subjected to (a) uniform and (b) random force directions with $F_{a p p}=0.6 \ldots \ldots \ldots$. . . . . . . . . . . . . . . . 


\begin{abstract}
Crucial in the exchange of information, may it be tangible or not, is mobility. From long-range migrations of populations to short-range movements in urban areas, communities operate in the context of the networks of interactions they are embedded in. The principal properties that define the attributes and evolution of systems are influenced by their mobility networks regardless of scale in space and time. In the long time scale of population migrations, we study the coevolution of languages and genes spanning centuries. Using cophylogeny techniques we investigate how population dispersals over thousands of years might have driven genetic admixture and linguistic transfer and speciation. Languages are transmitted through channels created by kinship systems. Persistence of these kinship channels leave traces in the genetic structure of a population. In the traditional societies of Sumba and Timor, movements among communities are driven by kinship practices which in turn shape both linguistic and genetic variations. Finely resolved co-phylogenies of languages and genes reveal persistent movements between stable speech communities facilitated by kinship channels that have structured genes and allowed for language transmission at small scales since the late Pleistocene. The association of languages and genes that has routinely been found from local to global scales can be explained as a result of this sustained adherence to kinship practices. In the short time scale characterized by high frequency of activities and fast activations of links, we model the mobility of commuters in a transport network. Interactions and movements in urban systems are controlled by their transport networks. For these systems to function efficiently, there is a need to build a robust network. We present here how we can couple land use and network structure to model the travel demand of stations and infer the mobility of commuters in a transport network. This gives us insights as to how transport networks should evolve with changing land use patterns and how this will affect commuter mobility. We apply the model to the Singapore Rapid Transit System (RTS). We find that considering the degree centrality of the network and using the gross plot ratio as the potential measure of how likely a commuter will be attracted to travel to specific areas, the predicted values of the model has a correlation of 0.70 with data. We further present a general model for defining the lifetime and resilience of spatial networks subjected to force loadings. Continuous use of the transport network at high throughputs can lead to a sizable number of
\end{abstract}


edges to break causing a network collapse. Some of the factors that influence the disconnection of links in evolving networks are force magnitudes, duration of time these forces are applied, and how nodes are connected and routes are directed in the system. Choosing the appropriate directionality of station connections (i.e. train or bus routes) can optimize the distribution of load in the system and can consequently make a network more resilient to failures. 


\section{Chapter 1}

\section{Introduction}

A universal vehicle immanent in the exchange of information, in both disembodied and tangible forms, is mobility [2,3]. Centuries of migration among communities in a population facilitates the transfer of collective knowledge and practices manifested as culture or expressed in genetics [3]. Similarly in the present setting, many of the tasks that need to be fulfilled in our everyday lives involve navigating an urban space [2]. Any urban system relies on the efficient trade of skills and goods, through continuous movement of individuals, to fulfil its various functions.

Communities in a traditional population or in an urban area operate according to the dynamical properties of its interconnected components. The principal characteristics that define any system can be described by tracking movements and interactions that transpire in space and time regardless of the scale, may it be migrations within and among populations in long time scales of thousands of years or daily individual movement through a city (Fig. 1.1).

By exploiting patterns of regularity, extracting the defining properties of a system is possible. For some cases, patterns of mobility is highly regular and striking like the trajectory of a projectile, planetary movements around the sun or other phenomena that may fall under the domain of classical mechanics. However, for systems of human mobility and migration, patterns are often vague and not easily discernible. But despite the many unpredictable factors that mask the necessary ingredient in formally describing mobility, we can still identify the distinctive features of a component of a system by exploiting the structure inherent in human movement[3].

Components of a complex system interact in the context of a structured network 


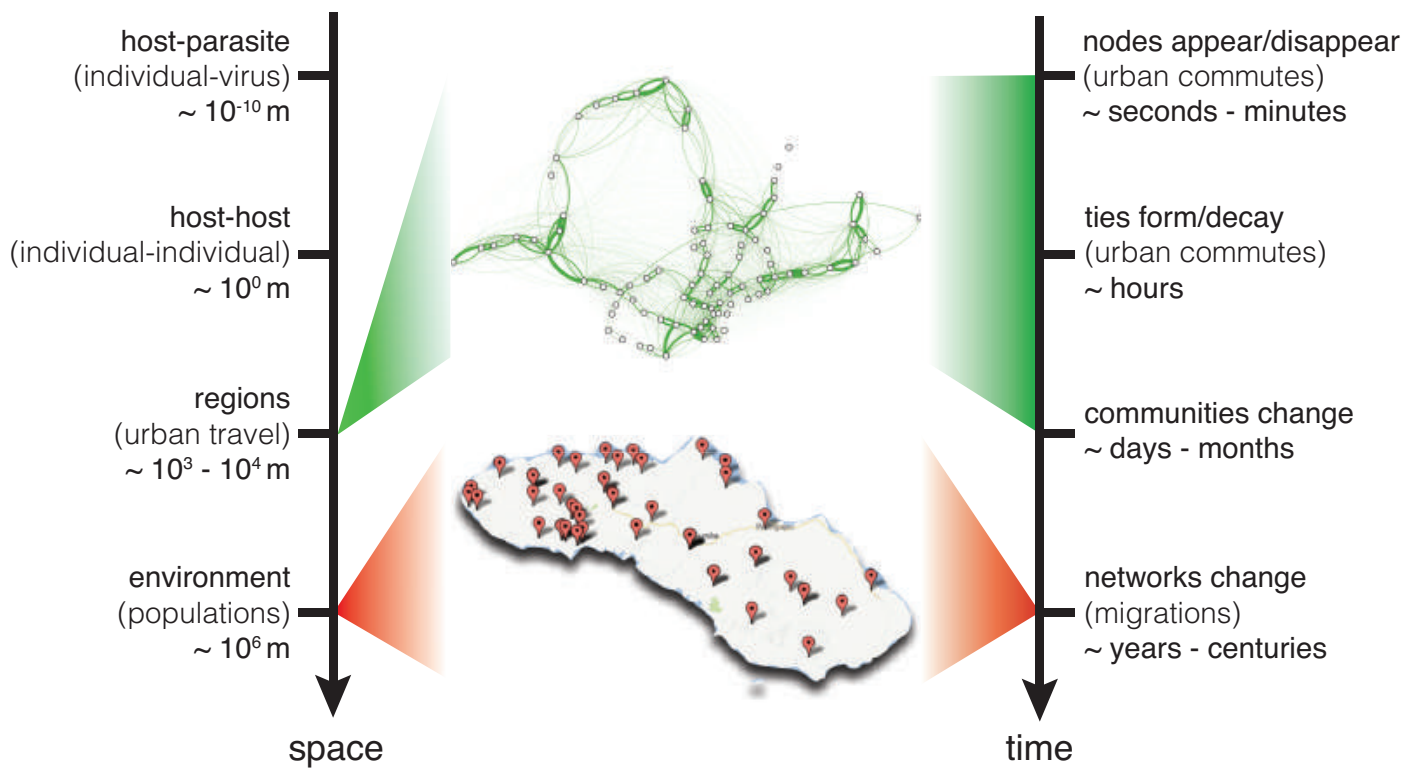

Figure 1.1: Spatial and temporal scales. Mobility is modelled for (1) population migrations (bottom red) that fall under a spatial scale of $\sim 10^{6} \mathrm{~m}$ and long time scales of years to centuries, and (2) urban travels (top green) involving regions of $\sim 10^{3}-10^{4} \mathrm{~m}$ and short time scales of seconds to days.

of mobility. Movements of humans are influenced by the networks they are embedded in. In traditional societies, post-marital residence rules create networks of migration that influences how much and who among the members of communities interact $[4,5]$. In chapters two to four, we explore how kinship practices create transmission channels that shape cultural and genetic diversity within and among populations. Similarly, the spatial distribution pattern of land use creates areas of attraction potential which generates a travel network that describes the degree and extent of individuals' movement in the urban space $[6,7,8]$. In chapter five, we present how the coupling of land use and transport network can describe travel demand in subspaces of an urban system.

Consequently, depending on our motion in the several layers of networks (i.e. social, cultural, infrastructure, geographic, transport) we live in, we might in turn also drive one of these networks to change. Social networks are dynamical by nature and with this comes a feedback loop wherein mobility patterns of agents cause network structure changes while the evolution of network structure also constrains the mobility of its agents. At different time scales, nodes may appear or disappear 
$[9,10]$, ties may form or decay $[11,12]$, and communities and the network itself may form, change or decay $[13,14]$. In chapter six, we study how a network dynamically evolve as forces that result from human mobility and interaction are applied [15].

In summary, we examine how mobility can be modelled by exploiting the inherent structure in human behaviour that exists in the context of the circumstance's spatial and time scale. In chapters two to four, we utilize the existence of cultural practices such as kinship rules to allow the modelling of how population migration patterns cause linguistic and genetic diversity in long (i.e. centuries) time scales. In chapters four and six, the use of urban space and the transportation structure are used to describe travel demand and resilience of networks in short (i.e. days) time scales.

\subsection{Population Mobility}

Some mobility networks develop and change over a relatively long time scale. Population migrations and genetic intermixing spans thousands of centuries. In slow rates of change, the movement of groups of people can cause a reconfiguration in their genetic makeup as well as in the composition of a population's cultural by-products.

Explicitly describing biological properties is often marred by noise and their dimly observed tendencies make it hardly possible to achieve a general consensus on how to define them [3]. Population genetics is special in this regard as the mechanism through which genetic information is inherited does possess an inherent structure [3]. The existing mathematics of population genetics can be used and extended to explore how a population will evolve under a set biological assumptions [3]. In chapter two, we extend Kingman's population mathematics to show how kinship practices drive changes in genetic diversity [3].

Population mobility does not only cause changes in genetic composition. During the interaction of different communities within a population, culture is also shared. This steers the evolution trajectories of cultural practices and by-products in various directions. In particular, it has been shown that in the global scale, migrations have driven co-evolution of genes and languages [4]. We show in chapter three how different migration patterns as defined by kinship affect the linguistic and genetic composition of a population.

The apparent strong association between the languages and genes can be further 


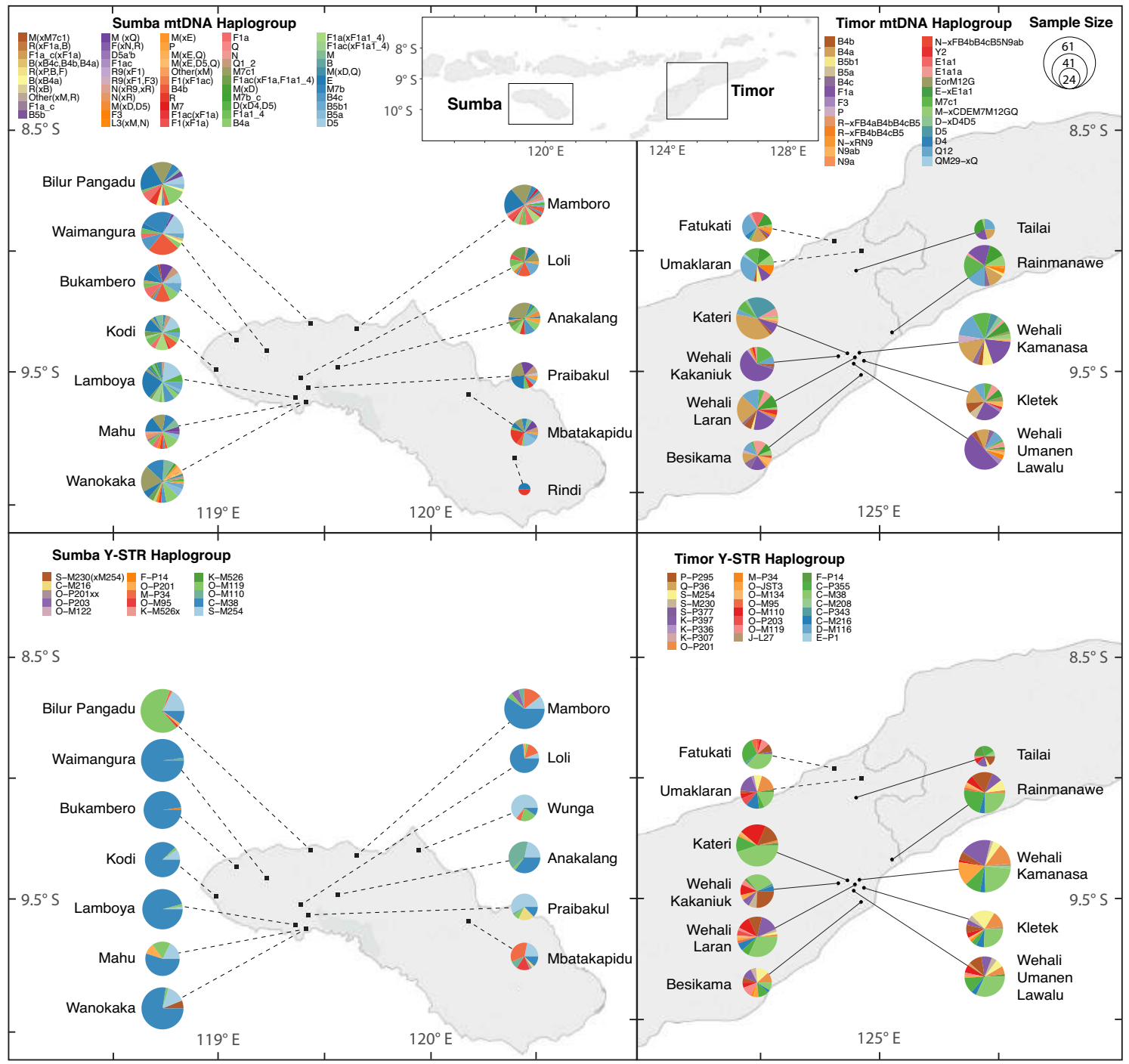

Figure 1.2: Genetic haplogroups in sampled villages of Sumba and Timor. Diversity of (Top) mtDNA haplogroups and (Bottom) Y chromosome STR haplogroups of villages in (Left) Sumba and (Right) Timor is shown with - - indicating the patrilocal villages and • indicating the matrilocal villages. Pie charts scaled by sample size show the distribution of haplogroups in the villages. 


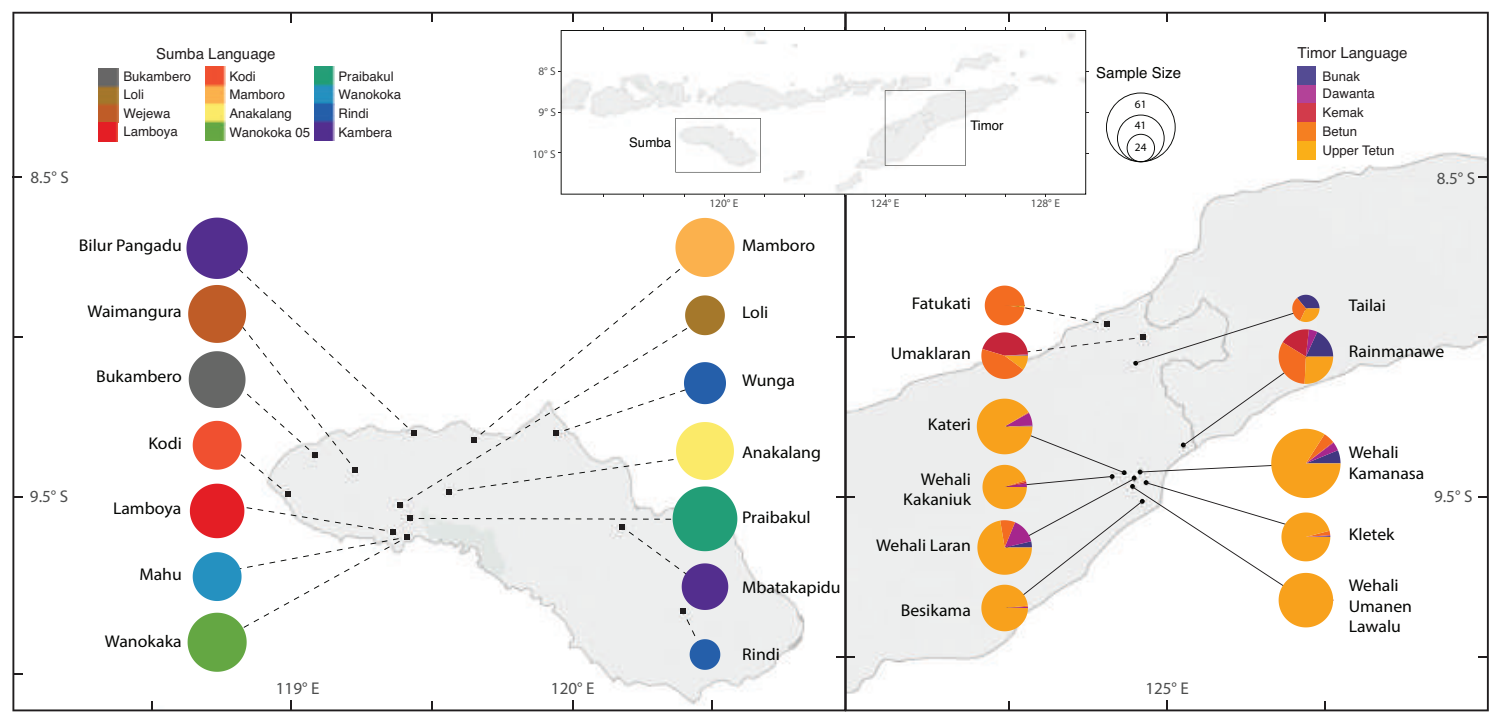

Figure 1.3: Languages in sampled villages on Sumba and Timor, eastern Indonesia. Pie charts show the languages spoken, scaled by sample size. Left: 14 patrilocal villages (-) on Sumba and the Austronesian languages spoken by the 505 sampled men. Right: 11 communities on Timor, including 9 matrilocal villages $(\bullet)$ and 2 patrilocal villages (-). Each of the 477 men sampled on Timor speaks one or more of five local languages belonging to two language families, Papuan (Bunak) and Austronesian (Betun, Dawanta, Kemak and Upper Tetun). Multiple languages are typically spoken within communities in Timor, while only one language is typically spoken within a given community in Sumba. 
tracked by looking at how mirroring of their phylogenies arise from events occurring at the community level [4]. As groups of people migrate to new villages, they bring with them their language. Any correlated change in the genetic and language networks of the "invaded" village must then point to a migration event happening at this point in history.

In developing a model of these mechanisms of population mobility, we use genetic (Fig. 1.2) and linguistic (Fig. 1.3) data from the islands of Sumba and Timor in Eastern Indonesia [4]. The team of Lansing et al. over a span of almost a decade has collected survey data and DNA samples showing the linguistic and genetic variations in Sumba and Timor which is a contact zone of Neolithic Austronesian farming communities and aboriginal foraging societies [4]. The migrating farmers began interacting with the resident hunter-gatherers around 3,500 years ago. By looking at the languages and genes of the sampled sub-population of Sumba and Timor, we can infer properties and events of mobility that led to the structure and distribution of the population's current genetic and linguistic composition.

The assemblage of published genetic data $[4,16,5,17,18]$, also used in this study, consists of mtDNA HVS-I sequences (positions 16001-16540 of the revised Cambridge Reference Sequence) and hierarchically screened SNPs on the Y chromosome, together with $14 \mathrm{Y}$ chromosome STRs. The language/s spoken by each individual is determined by asking their level of comprehension for each of the language recorded in their village. Men on Sumba are monolingual and speak one of 12 Austronesian languages. Each man sampled on Timor speaks one or more of five local languages belonging to two language families, Papuan (Bunak) and Austronesian (Betun, Dawanta, Kemak and Upper Tetun).

\section{$1.2 \quad$ Urban Mobility}

In the time scale of days or weeks, fast link activations and deactivations may take place in a mobility network depending upon the number of events and agent contacts that happen within it [19]. Links of specific attributes are active for certain periods if they provide the parameters needed for a process to occur. In the same way, travel routes available through transportation networks are operative at varying degrees throughout the day depending upon the commuters' need to travel to the areas they 


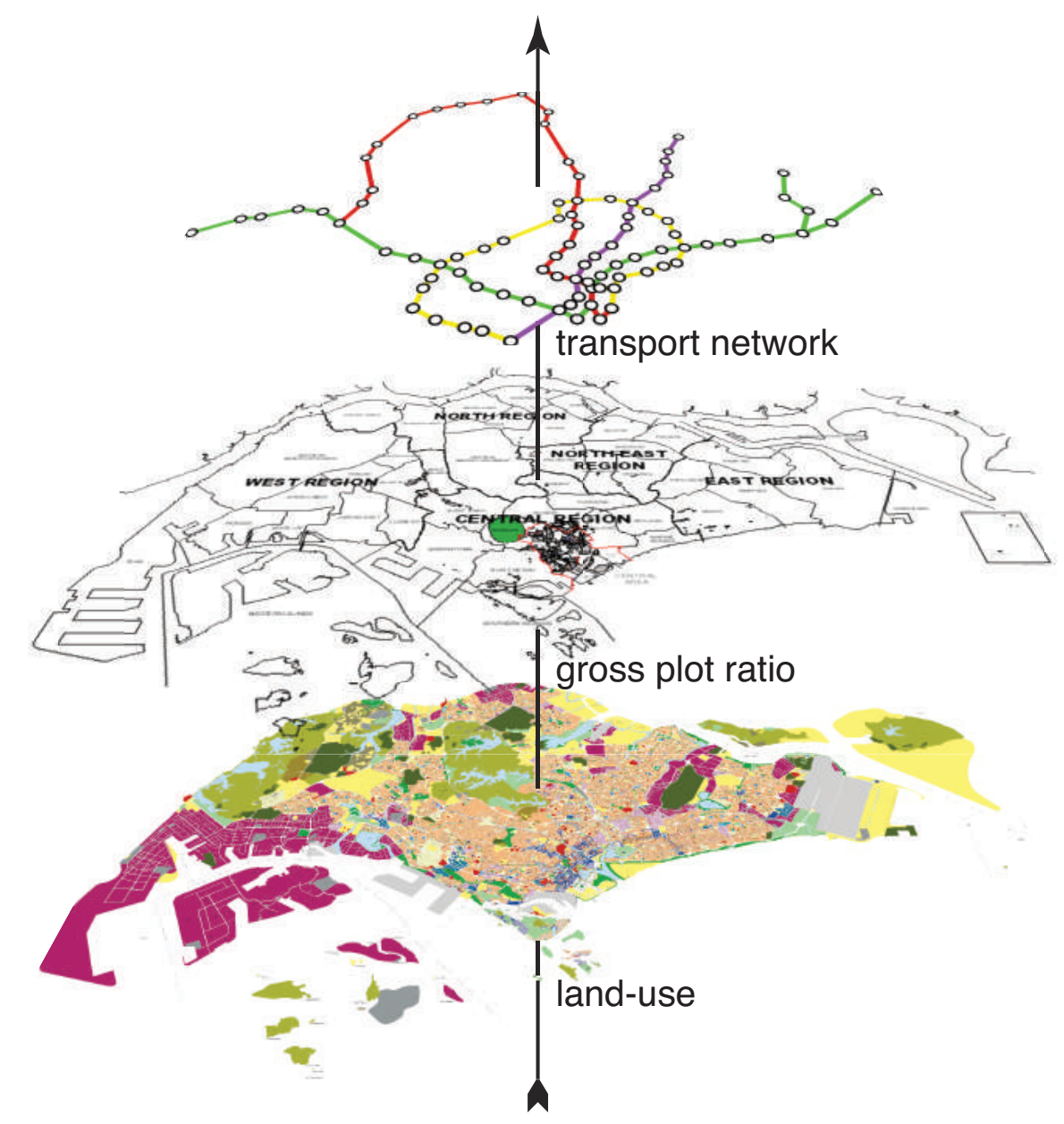

Figure 1.4: Urban mobility network layers. Mobility events in urban areas are defined by the coupling of land-use entities in the urban planning map, gross plot ratio proxy of population density and transport network attributes.

connect.

Transportation and urban mobility, being the primary drivers of human interaction controls the economy and ability of an urban system to support its dwellers. Public transport systems, because of their ability to move individuals at higher volumes and frequency but with less resources has become the primary mode of transport in urban areas. In chapter five, we show how the characteristics and design of a city influence how its inhabitants navigate the space. Three important factors that play into how we move around a city are space use, population density and transport topology. As shown in Fig. 1.5, these three parameters can be used to determine how and where we travel.

We study mobility patterns in short time scales in the context of the Singapore 
Rapid Transit System (RTS). The travel demand of each node in the RTS network is influenced by the type and density of the land-use entities in the region it serves. These parameters are obtained from the Urban Redevelopment Authority (URA) planning map which defines the kinds of amenities (Fig. 1.5 land-use layer) and how much of them (Fig. 1.5 gross plot ratio layer) can be developed in every parcel of land in the region. To define the mobility events in the transport network of Singapore we couple the RTS network with the land-use and gross plot ratio maps which define the locations of the entities (i.e. residential, business, industrial, education) in the country. Most of the journeys in a transport network involve travels from residential to work or education areas and vice versa. Since these processes are more prevalent and are predictable, we concentrate on these mobility events.

Continuous use of transport networks and prolonged application of demand force can cause congestion and travel interruptions. In chapter six, we present a general method through which we can explore fragmentation and resilience of any kind of network subjected directly or indirectly to either physical or abstract forces [15]. A stochastic fatigue model can give insights on the lifetime of transport networks which allows a way to measure robustness. We show how this general method can be applied in modelling the evolution of the Singapore bus network as travel demand forces are applied to its components.

Fractures and fragmentation happen in a large variety of physical, biological, and geological systems. Fracture in these systems have been considerably studied. But one can also imagine fracture not just in the context of materials. Fracture also happens in other networks like road, protein, power grid and neural networks. Nodes here are not molecules or atoms but macroscopic entities instead. Force and loading that lead to fracture exist in these systems in the form of vehicular or energy flows or strength of interactions between nodes.

In all these classes of systems, one thing is true. Memory is important. Whether a particular link will fail or not depends not only on the instantaneous deformation resulting from the force a node applies on another at the current time. But it remembers all of the past forces it has experienced. This damage accumulation term introduces memory over the loading history of the system. Damage accumulation can eventually lead to network collapse. As an example we show in chapter six how a transport network may fragment into many small clusters wherein congestions result 


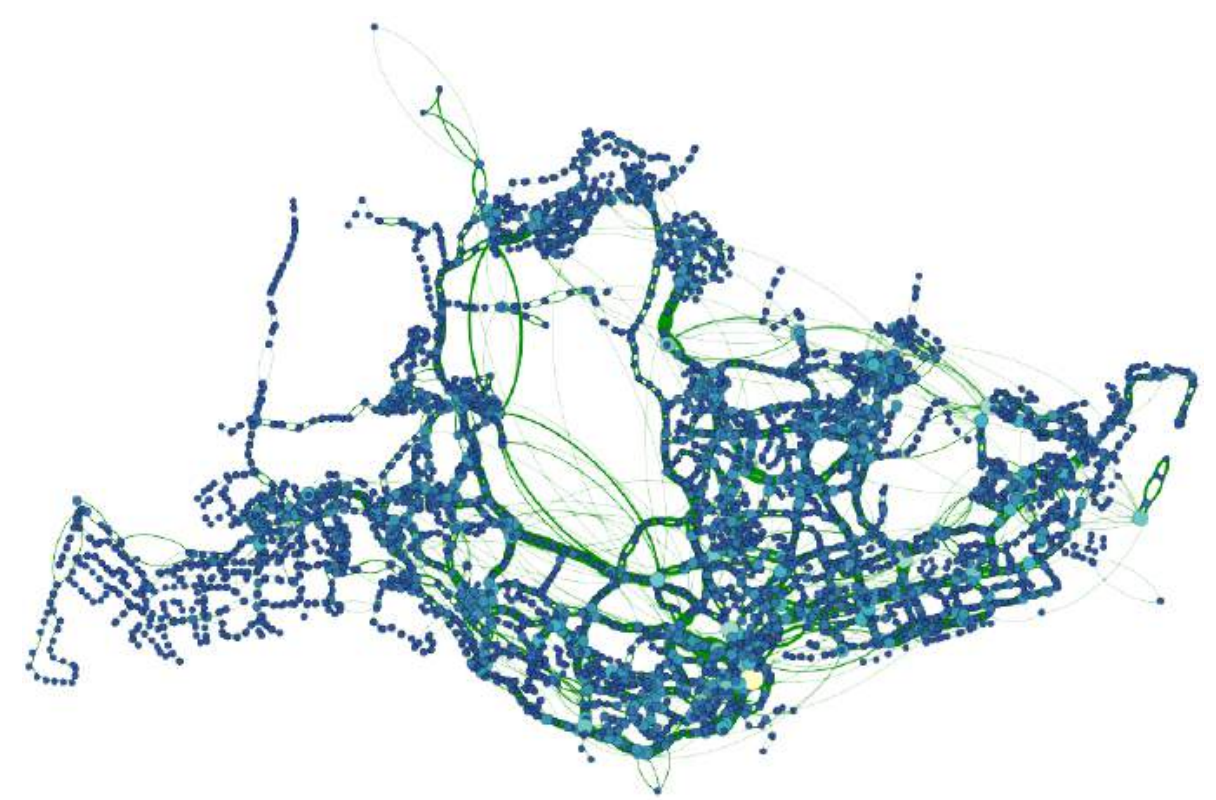

Figure 1.5: Travel demand forces. A stochastic fatigue model is used to follow the evolution of the Singapore bus network as travel demand forces cause strain along its network links. Bigger and lighter nodes are the often used bus stops. Links are thicker if demand for the routes is higher.

to long travel times causing some parts of a region to be momentarily isolated.

\subsection{Aims of the study}

We endeavour to find spatio-temporal models that can explain the mobilities observed in (1) rural or traditional regions, and (2) in urban areas. Since the nature of the movements in these two systems are inherently different, we do not aim to present a unifying framework for both, but instead we show how the different and unique characteristics of these systems can be dynamically captured.

For the population mobility studies, with help from geneticist collaborators (Guy Jacobs and Elsa Guillot) who assisted in constructing the genetic trees and distances and a linguist collaborator (Sean Downey) who helped construct the language trees and distances, we present how kinship systems serve as migration channels through which genes and languages co-evolve. Associations between genes and languages have been found even in the presence of sustained migration among communities. We compare phylogenies of gene and language trees to explore the origins of this 
association. In traditional tribal societies, marriage customs channel language transmission. When women remain in their natal community and men disperse (matrilocality), children learn the language of their mothers, and language correlates with the maternally inherited mitochondrial DNA. When men stay in their natal community and women disperse (patrilocality), the opposite pattern holds. Kinship rules that dictate postmarital residence can persist for many generations, and determine population genetic structure at the community scale. The association of languages with clades created by kinship systems provides information not only about language transmission, but also about the structure and persistence of social groups.

For the urban mobility studies, the candidate performed all the analysis presented with some help from an A*STAR collaborator (Erika Fille Legara) who analyzed the raw transport data. Urban systems have the signature characteristic of intense interactions resulting from high-frequency movements of its inhabitants as enabled by an efficient and robust Rapid Transit System (RTS). While predictive models on human mobility are available at the scale of inter- and intra-country level, there is as yet few successful models that predict human movements at the urban scale. In this study, we proposed a modified radiation model that is able to estimate with good accuracy the average mobility flux between the origin-destination stations of a real-world RTS. We achieved this by enhancing the original radiation model to incorporate information on land-use from the urban plan and coupling it with the RTS network structure. The inclusion of these details into the radiation model provides finer-scale information which accounts for the effect of spatial heterogeneity not possible in the original model. We apply the model to the Singapore Mass Rapid Transit (MRT) system by considering its degree centrality and using the gross plot ratio weighted by spatial entropy to determine how likely a commuter is attracted to travel to specific areas. Using a model of network fragmentation, we then aim to show how a transport network will evolve in time as a result of these travels that exerct forces within the network's interacting nodes. 


\section{Chapter 2}

\section{Genetic Diversity}

Started out from Africa around 60,000 years ago, humans are known to have constantly migrated from one place to another throughout history. Human migration, motivated by diverse reasons, shows complex patterns that may vary at different scales. Here, we are interested in migrations motivated by marriages. Indeed, matrimonial migration is by far the largest form of migration in most indigenous human societies where individuals are found to leave their community of origin to marry and live in another community. Such migrations are often non-random. An individual is often opted to mate with another specific individual in a selected group to forge alliances and unite territories. And our genes vary according to our mating patterns. In other words, the anthropological tradition of kinship and post-marital residence rules naturally structures the genetic patterns of local populations. If adherence to marriage rules is carried for many generations, strong constraints on genetic heterozygosity are imposed. The degree and extent of inbreeding and outbreeding as dictated by the social regulations of kinship practices will largely reflect on a population's genetic diversity. Being one of the oldest sources of information on how genes is passed down to the next generation, kinship systems provide a context through which we can resolve contentions on the cause of genetic diversity, at least on the regional scale.

DNA based techniques are often used to study kinship systems. Nonetheless, genetic data often shows complex pattern which cannot be explained based on our current understanding on genes and mechanisms that underlaid its evolution. If we look at the world's genetic landscape after the New World was discovered in 1492, America seems to have accumulated the highest levels of allele diversity [20]. 
Out-of-Africa theorists have also routinely used African American populations as proxies to native Africans because they are genetically closely similar [20, 21]. This might lead anyone unfamiliar with world history to conclude that the origin of humans is the Americas. But much of what the genetics in America tells us is a result of the European and African colonization of the New World. Until recently, it is widely believed that modern humans came out of Africa. This theory is now being challenged after it was discovered that 1-3\% of Eurasian genome is of Neanderthal origin [21]. But, still bulk of the variations has an African origin. It has therefore recently been modified to accommodate some introgression of Archaic Homo from outside of Africa. Genetic data alone is not always clear as to where modern humans originated [20]. Anthropological events and practices that have caused both sweeping and local migrations have to be included in models of genetic diversity to resolve the directionality of human movements beyond ambiguity [20].

There is thus a growing literature on the employment of various modelings for the studies of kinship systems. Agent-based modeling is one of which involves computational simulations on the actions and interactions of agents who obey simple rules, in this case, the unilateral post-marital rule. By evolving actions of the agents, effects of such rules can be studied on the system as a whole. Here, we are interested in employing the agent-based model to study the influence of unilateral post-marital residence rules on genetic diversity.

Levi-Strauss formally classified post-marital residence rules [22] and kinship systems (Table 2.1) into (1) patrilocal (men stay in their natal community and women disperse), (2) matrilocal (women stay in their natal community and men disperse, (3) neolocal (both men and women disperse) and (4) endogamy (men and women marry within their natal community).

Early Austronesian societies are found to practice matrilocal post-marital residence where the husband lives with the wife's kin [23]. In contrast, Indian women move from their natal villages almost all on marriage [24]. Similarly, villages in Sumba are known to practice patrilocality [4]. Africa, on the other hand, has mostly strict patrilocal or matrilocal residence $[22,25]$. As such, patrilocal and matrilocal communities are often modeled and studied separately. Patrilocal kinship practices are shown to have influenced the dispersion of genetic markers differently from matrilocal kinship practices. In particular, the loss of gene diversity due to genetic 
Table 2.1: Kinship and post-marital residence rules. Culturally prescribed rules of residence post marriage is described below.

\begin{tabular}{c|cc} 
Kinship Practice & Men & Women \\
\hline Patrilocal & stay in natal village & disperse \\
Matrilocal & disperse & stay in natal village \\
Neolocal & disperse & disperse \\
Endogamy & stay in natal village & stay in natal village
\end{tabular}

drift is shown to be slower in populations which pursue matrilateral rather than patrilateral cross-cousin marriages [26]. On the other hand, Asymmetric Prescriptive Alliance (APA) marriage rules are shown to influence genetic diversity on the autosomes, X chromosomes and mitochondrial DNA (mtDNA), but not the paternally inherited $\mathrm{Y}$ chromosome in a group of interacting patrilocal clans [18]. In regions consist of villages with unilateral kinship practice, patrilocal post-marital residence is known to create diversity in the population's mtDNA while matrilocal post-marital residence is known to create diversity in the population's Y chromosome. As such, high relative patrilineal correlation is often taken as a signature of patrilocal residences in which males have stayed for generations. High correlation in the matrilineal gene, on the other hand, is taken as a signature of matrilocal residences.

In this chapter, we do agent-based modelings on communities practiced unilateral post-marital residence rules and kinship systems as classified in table 1. Unlike previous studies, we introduce inter-residence interaction instead of strict intraresidence interaction into our model. With this, communities which follow different kinship practices are allowed to interaction with each other. Incorporation of such inter-residence interaction are motivated by our findings in a group of villages in Timor which interacts actively among themselves though kinship rules practiced by them are different. Here, in a patrilocal village, men choose to stay while women can migrate either to another patrilocal village or a matrilocal village for marriages. The same happens to men from matrilocal villages who leave their natal communities for marriages. Under such a setting, we investigate fingerprints of patrilocal versus matrilocal residence rules. 


\subsection{Genetic Tree}

The dataset comprises 982 Indonesian men from the eastern Indonesian islands of Sumba $(n=505)$ and Timor $(n=477)$, with associated genetic and linguistic information. Of the 25 villages in this study, 14 are on Sumba and 11 on Timor. All Sumba villages are patrilocal; while of the 11 Timor villages, two follow patrilocal kinship practice (Fatukati and Umaklaran), and the remainder are matrilocal. Genetic information was derived from peripheral blood and buccal samples. The diversity of haplogroups present in each sampled village is shown in Fig. 1.2. The genetic trees were constructed using the method explained below. This method to construct genetic trees using haplogroup, and HVRI for mtDNA or STR for $\mathrm{Y}$ chromosome is freely available as an opensource $\mathrm{R}$ application on https://carpntrees.shinyapps.io/shinyapp/.

\subsection{1 mtDNA tree and distances}

The female lineage tree was constructed using mitochondrial DNA data, using a combination of the HVR1 sequence (540 base pairs) and haplogroup information. While the HVR1 expresses genetic diversity that represents recent variation between individuals, the haplogroups (determined by hierarchically genotyping SNPs) permit the high accuracy resolution of deep lineage phylogeny. By combining both sets of information we can obtain a phylogenetic tree that far better represents the true phylogeny than is possible with only one data source.

The method involves several steps. First a tree is built using the the haplogroup of each individuals, with a typology matching the consensus haplogroup tree from the Phylotree project [27]. This tree contains leaves with individuals sharing the same haplogroup. Such leaves are then sorted using a neighbour-joining algorithm on the HVR1 sequence, using the most closely related haplogroup as an outgroup. Thus, we iteratively generate a final tree in which the deep nodes are determined by haplogroup and recent nodes by HVR1. All individuals that present exactly the same genetic information on the mtDNA (i.e. same HVR1 sequence and same haplogroup) are clustered together to speed up the calculations, and separated at the tip after the tree reconstruction is complete. The final tree is shown in Fig. 2.1. Individual population trees are extracted from this overall tree. The pairwise dis- 
tance matrix used for IM model fitting and language transmission analysis was built using the number of pairwise differences in the HVR1 sequence between each pair of individuals.
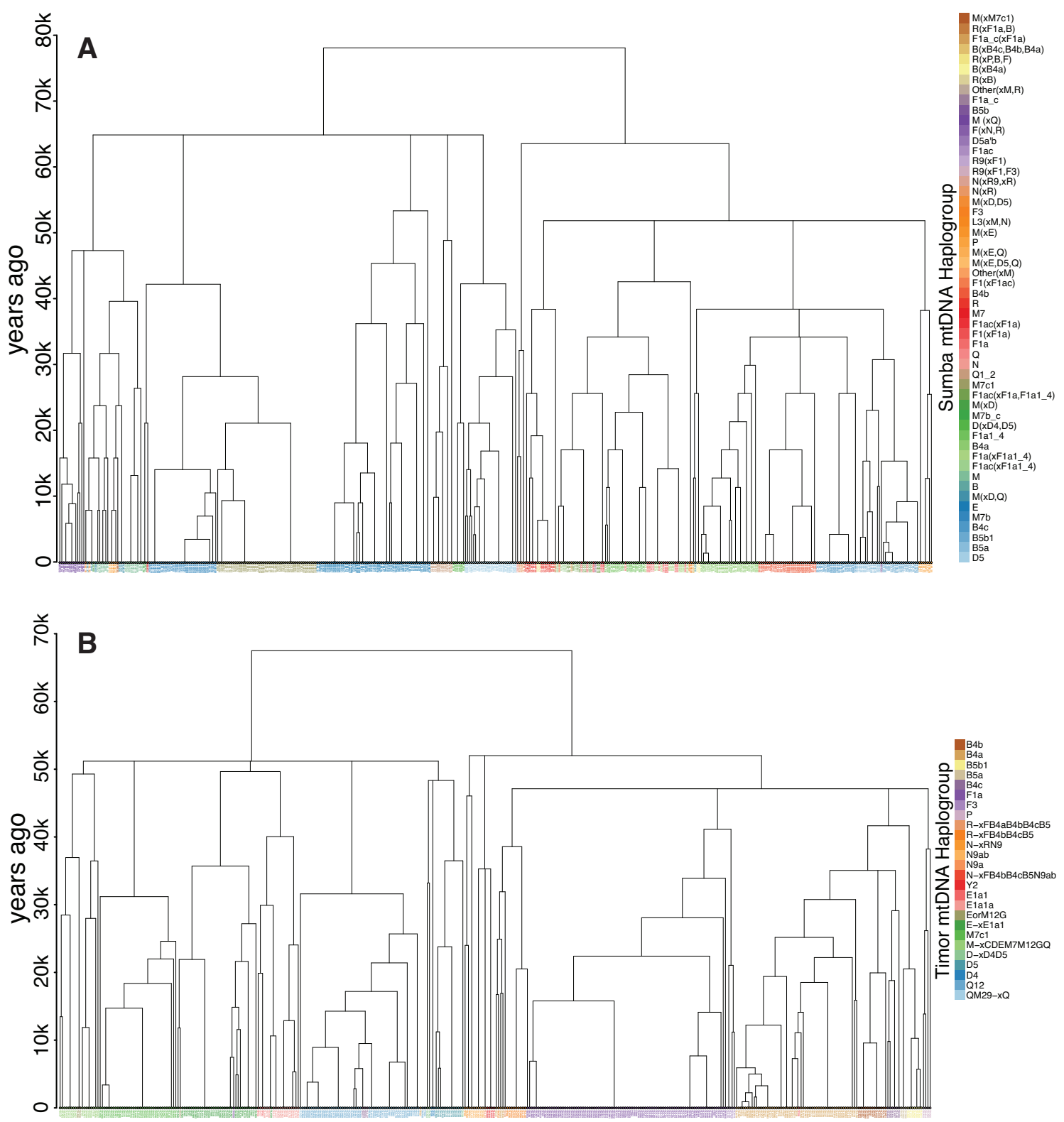

Figure 2.1: mtDNA genetic tree. The female lineage of the sampled Indonesian men from (A) Sumba and (B) Timor colored according to their mtDNA haplogroup. 


\subsubsection{Y chromosome STR Tree}

The male lineage tree was reconstructed using both Y-STR markers and Y chromosome haplogroup data. Using a similar method as for mtDNA, both sets of information were combined by first building a haplogroup tree and then sorting recent lineages using Y-STRs. The haplogroup tree was this time built to have a topology matching the Y-DNA Haplogroup Tree of the International Society of Genetic Genealogy [28], with refinement based on Karafet et al [29] to incorporate markers specific to that study. To sort the tips, the Bruvos's distance [30] between each pair of individuals was calculated based on their Y-STRs. As before, leaves of the haplogroup tree containing multiple leaves were then sorted using a neighbourjoining algorithm, this time on the Bruvo's distance of the Y-STRs. As before, all individuals with the same genetic information were treated together and separated at the tips of the tree after the tree was constructed. The final tree is shown in Fig.2.2, and individual population trees were extracted from this overall tree. The pairwise distance matrix used for IM model fitting and language transmission analysis was built using the Bruvos distance between all pairs of individuals calculated based on their Y-STRs.

\subsubsection{Molecular Dating of The Tree}

We use haplogroup dating information with confidence intervals to calibrate the trees. On the female tree, three time points are used which are issued from whole sequence molecular dating by $\mathrm{Fu}$ et al. [31]. On the male tree, five time points are used taken from a publication by Karafet et al. [29]. Molecular dating of both trees using these calibrations points was done using the function chronos of the $R$ ape package [32, 33].

\subsection{Two-deme Model of Migration in a Struc- tured Population}

To study the characteristic changes in the biology of a spatially divided population, Kingman in 1982 developed a Markov chain model to describe the genealogy of individuals drawn from a large haploid population [34]. Similar to his model, we 

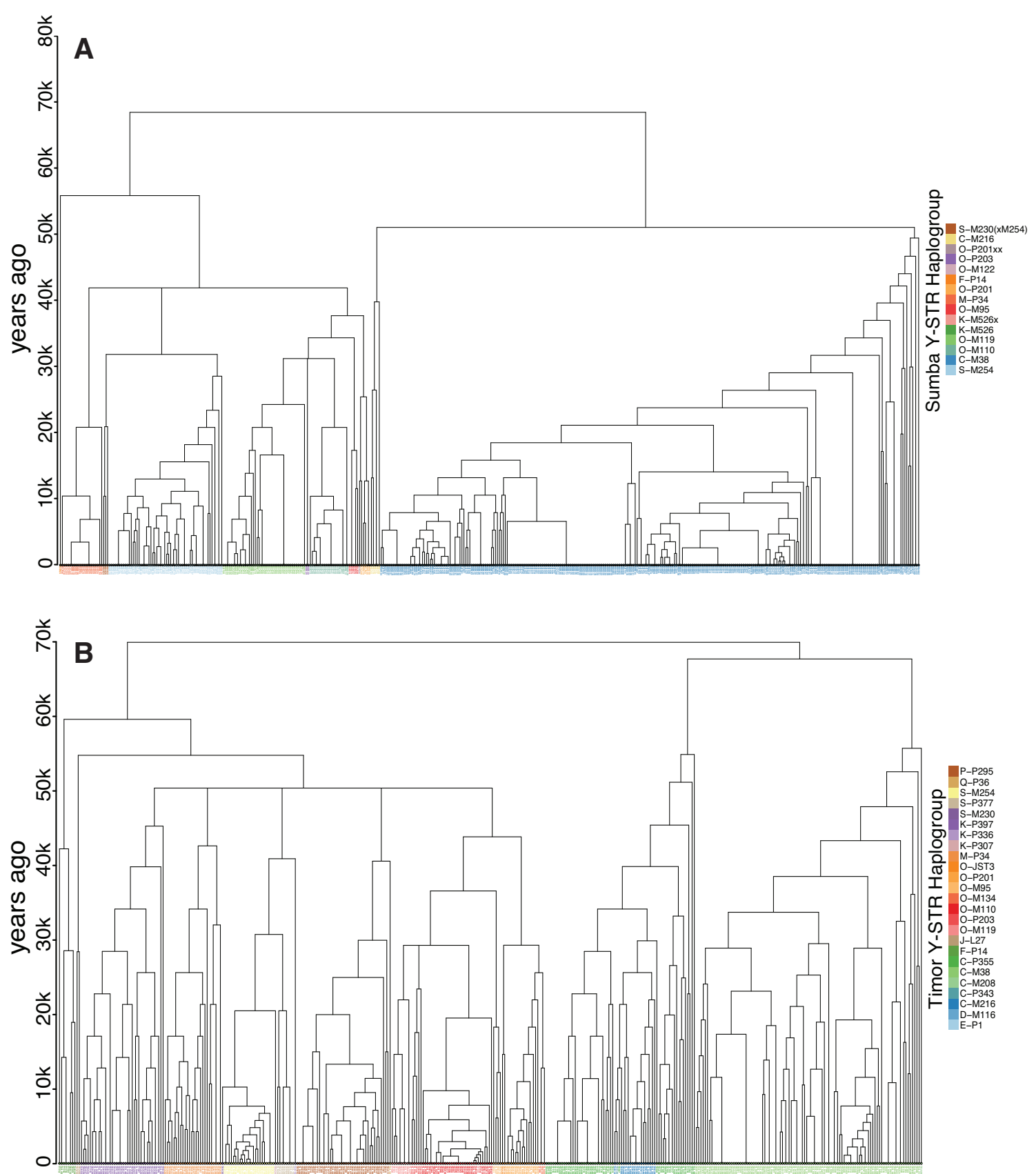

Figure 2.2: Y chromosome STR genetic tree. The male lineage of the sampled Indonesian men from (A) Sumba and (B) Timor colored according to their Y chromosome STR haplotype. 
trace uniparental descent and consider only the transfer of haploid gametes from one generation to the next [34]. mtDNA and Y chromosome are effectively carried along only one line of descent with mtDNA inherited from mothers and Y chromosome from fathers. In this work, we look at how mtDNA and Y chromosome diversity vary according to the followed kinship practice. We focus on the degree of mixing of different family clusters as individuals move from one village to another following their local customs. For this purpose, we ignore mutation and selection and consider genes (mtDNA and Y chromosome) labelled by their village membership at generation 0. Every male carries two gene labels corresponding to his mother's village mtDNA and father's village Y chromosome while every female carries only one gene label corresponding to her mother's village mtDNA.

We consider a Wright-Fisher population of discrete and non-overlapping generations with a constant size of $N$ individuals [35]. To maintain a constant population size, for every one individual (male or female depending on kinship system) that moves at time $t$, we assume that (1) for every male/female that leaves a village, a male/female will be born inbred by a randomly chosen couple, (2) for every male/female that moves into a village, a male/female dies, and (3) at the next generation, every couple is replaced by their son and daughter. In (3), at time $t+1$, the father is replaced by a male carrying the father's Y chromosome and the mother's mtDNA, and the mother is replaced by a female carrying her mtDNA. In addition, every subpopulation is panmictic. Individuals from the same village at generation $g$ are interchangeable. For a two-deme population, the dynamics of movement is summarized in Fig. 2.3.

\subsubsection{Diversity comparison of uniparentally inherited genes}

Depending on the kinship practice, the direction and magnitude of male and female migrations may vary. Hence, the degree of relatedness along the father line and the mother line of individuals in a village may vary. We define $\rho_{m m}(t)$ as the probability that any pair of individuals have the same female ancestor at time $t$ and $\rho_{y y}(t)$ as the probability that any pair of individuals have the same male ancestor at time $t$. Normalizing, we get 

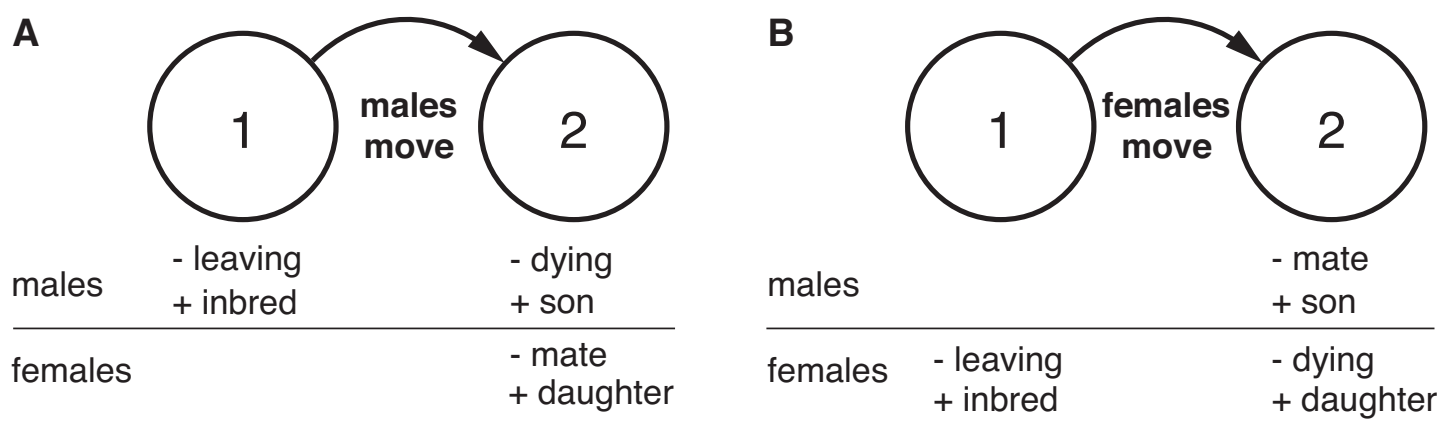

Figure 2.3: Movement assumptions for agent-based model of kinship-driven migrations between structured populations. Schematic diagram of the rules followed by every individual that moves per time step in the agent-based model is shown. For every pair of interacting demes (shown as circles), every individual that move (represented by the arrows) is replaced by a new individual randomly chosen from the subpopulation's existing pool. A - in the table below the circles indicate the subtraction of one male/female and $\mathrm{a}+$ is an addition of one male/female in the deme (circle 1 or circle 2). (A) and (B) shows that a male/female moving out of subpopulation 1 (indicated by -leaving) is replaced by a male/female bred from a randomly chosen male and female in sub-population 1 (indicated by +inbred). A randomly chosen male/female in sub-population 2 (indicated by -dying) is replaced by the immigrating male/female. The immigrant and his randomly chosen mate (indicated by - mate ) is then replaced by their son (indicated by + son )and daughter (indicated by +daughter). 


$$
\begin{aligned}
p_{m m}(t) & =\frac{\rho_{m m}(t)}{\rho_{m m}(t)+\rho_{y y}(t)}, \\
p_{y y}(t) & =\frac{\rho_{y y}(t)}{\rho_{m m}(t)+\rho_{y y}(t)} .
\end{aligned}
$$

To allow tracing of both parental descents, we follow both male and female migrations but consider only pairwise comparisons of male $p_{m m}$ and $p_{y y}$.

If a village is patrilocal, men stay in their natal village while women may disperse. We expect then that $p_{y y} \geqslant p_{m m}$. A matrilocal village, on the other hand, is composed of women that are local and men that are either local or immigrants. Hence, $p_{m m} \geqslant$ $p_{y y}$. A neolocal village wherein a newly wed couple can reside separately from the husband's and the wife's natal village can either be more related in the matriline or the patriline depending on the type of genes the immigrants replace in the subpopulation.

In attempting to predict the matrilineal and patrilineal genetic diversity of subpopulations, villages cannot be considered in isolation. The amount of genetic mixing results from the network of interactions of all the villages in the population. If all demes in a population follows only one kind of kinship practice, the above relations are expected to hold - that is, $p_{y y} \geqslant p_{m m}$ for a patrilocal kinship system and $p_{m m} \geqslant p_{y y}$ for a matrilocal kinship system.

For a population subdivided to only two demes, the relation of $p_{m m}$ to $p_{y y}$ is easily tractable. In Fig. 2.4, we present the degree of mtDNA and Y chromosome diversity after one generation for a two-deme population with each sub-populations initialized to contain only one kind of each gene, $\left(m t_{i}, y_{i}\right)$ for males and $m t_{i}$ for females where $i \in\{1,2\}$ is the village label. Suppose that we do not initially know the kinship practice of a village. We can look at the value of $p_{m m}-p_{y y}$ in the village to decide what type of post-marital residence rule it follows. We define $p_{m m}-p_{y y}<0$ as a "patrilocal" signal and $p_{m m}-p_{y y}>0$ as a "matrilocal" signal. Indeed, we see that for the homogeneous kinship practice interactions (i.e. patrilocal - patrilocal in Fig. 2.4A and matrilocal-matrilocal in Fig. 2.4B), the deduced signal is equivalent to the villages' actual kinship practice. A network of only patrilocal villages will always have only local males thus preserving the purity of their Y chromosomes while a network of only matrilocal villages will not have male immigrants resulting to the conservation of the villages' original mtDNA. 

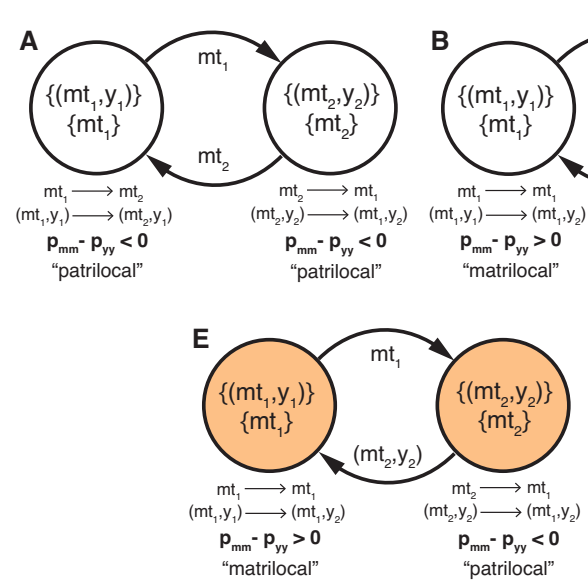
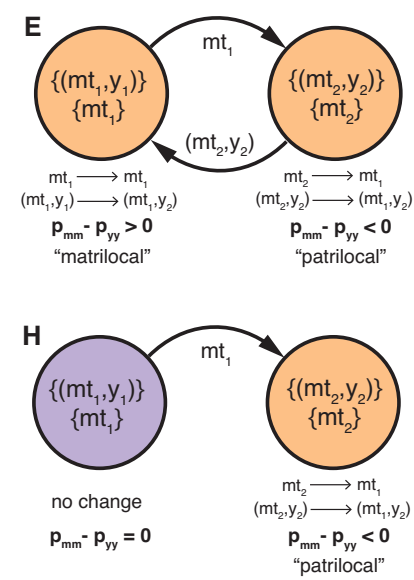

$\mathbf{F}$
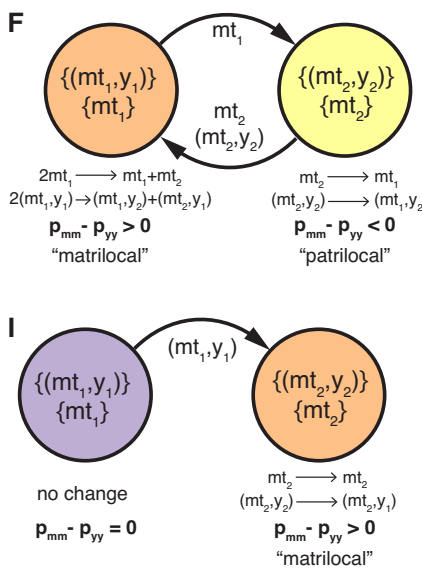

C
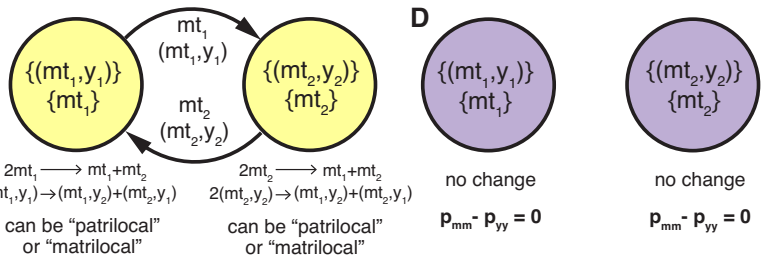

no change

$p_{m m}-p_{y y}=0$

Figure 2.4: Two-deme kinship-structured migration interaction. The top row shows the replacement of mtDNA $m t_{i}$ and $\mathrm{Y}$ chromosome $y_{i}$ after one migration event when both demes have the same kinship practice. (A) Patrilocal-patrilocal interaction preserves $y_{i}$ deme homozygosity while (B) matrilocal-matrilocal interaction preserves $m t_{i}$ deme homozygosity. (C) Neolocal-neolocal can stochastically show either a "patrilocal" or a "matrilocal" signal. (D) Endogamous interactions mantain the original $m t_{i}$ and $y_{i}$ compositions of the demes. The second row shows bidirectional interactions of villages heterogeneous in kinship practice. (E) Patrilocalmatrilocal interaction causes villages to show a signal opposite to its actual kinship practice. (F) Patrilocal-neolocal and (G) matrilocal-neolocal interactions results to the neolocal village adopting the signal of the other village which in turn crossdresses. The bottom row of unidirectional migration shows $(\mathrm{H})$ patrilocal-endogamy, (I) matrilocal-endogamy and $(\mathrm{J})$ neolocal-endogamy interactions. In $(\mathrm{H})-(\mathrm{J})$, nonendogamous villages show no change in genetic composition while endogamous villages give the signal of the village it interacts with. White villages emits a signal similar of its actual kinship practice, orange villages are cross-dressers, yellow villages are neolocal villages that can either be "matrilocal" or "patrilocal" while violet villages maintain their original genetic composition. 
A neolocal village exchanging both husbands and wives with another neolocal village as depicted in Fig. 2.4C can either exhibit a "patrilocal" or a "matrilocal" signal depending on the stochastic movement of males and females. On the other hand, as shown in Fig. 2.4D, no flow of either male or female happens when an endogamous village interacts with another endogamous village resulting to no change in the initial genetic composition of the sub-populations.

In Fig. 2.4E, we investigate what happens when the kinship practice of the two demes is not the same. Consider the case when village 1 is patrilocal and village 2 is matrilocal. Village 1 will always be the source of female immigrants while village 2 is the source of male immigrants. Since village 1 has purely female emigrants and purely male immigrants, men will always have the original mtDNA, $m t_{1}$, but the $\mathrm{Y}$ chromosome can either be from a local or an immigrant resulting to $p_{m m}-p_{y y}>0$. A village that in reality practices patrilocality appears to be "matrilocal". Similarly for village 2 , since only males emigrate and only females immigrate, men will always have the original $\mathrm{Y}$ chromosome, $y_{2}$, but mtDNA will vary. A $p_{m m}-p_{y y}<0$ signal will fool an uninformed observer into thinking that the matrilocal village 2 is "patrilocal". This heterogeneous interaction of kinship practices gives rise to the phenomena of trans-locality wherein the villages appear to be cross-dressers whose signal of kinship locality is different from its actual post-marital residence custom.

The interaction of a neolocal village with either a patrilocal (Fig. 2.4F) or a matrilocal (Fig. 2.4G) village results to the neolocal village dressing like the patrilocal or matrilocal village it exchanges migrants with. The patrilocal village as a result of having a net inflow of male immigrant appears to be "matrilocal" (Fig. 2.4F) while the net inflow of females causes the matrilocal village to cross-dress as a "patrilocal" (Fig. 2.4G). A unidirectional migration takes place when one of the villages is endogamous. As shown in Figs. 2.4H-J, this results to the non-endogamous village retaining its original genetic composition as no foreign gene is added to its pool. The endogamous village, however, puts on the dress of the other village and appears as either "patrilocal" or "matrilocal".

\subsubsection{Two-deme simulation of kinship-structured migrations}

Investigating whether the above results hold beyond the first migration event, we simulate the exchange of husbands and wives of two villages with a defined kinship 

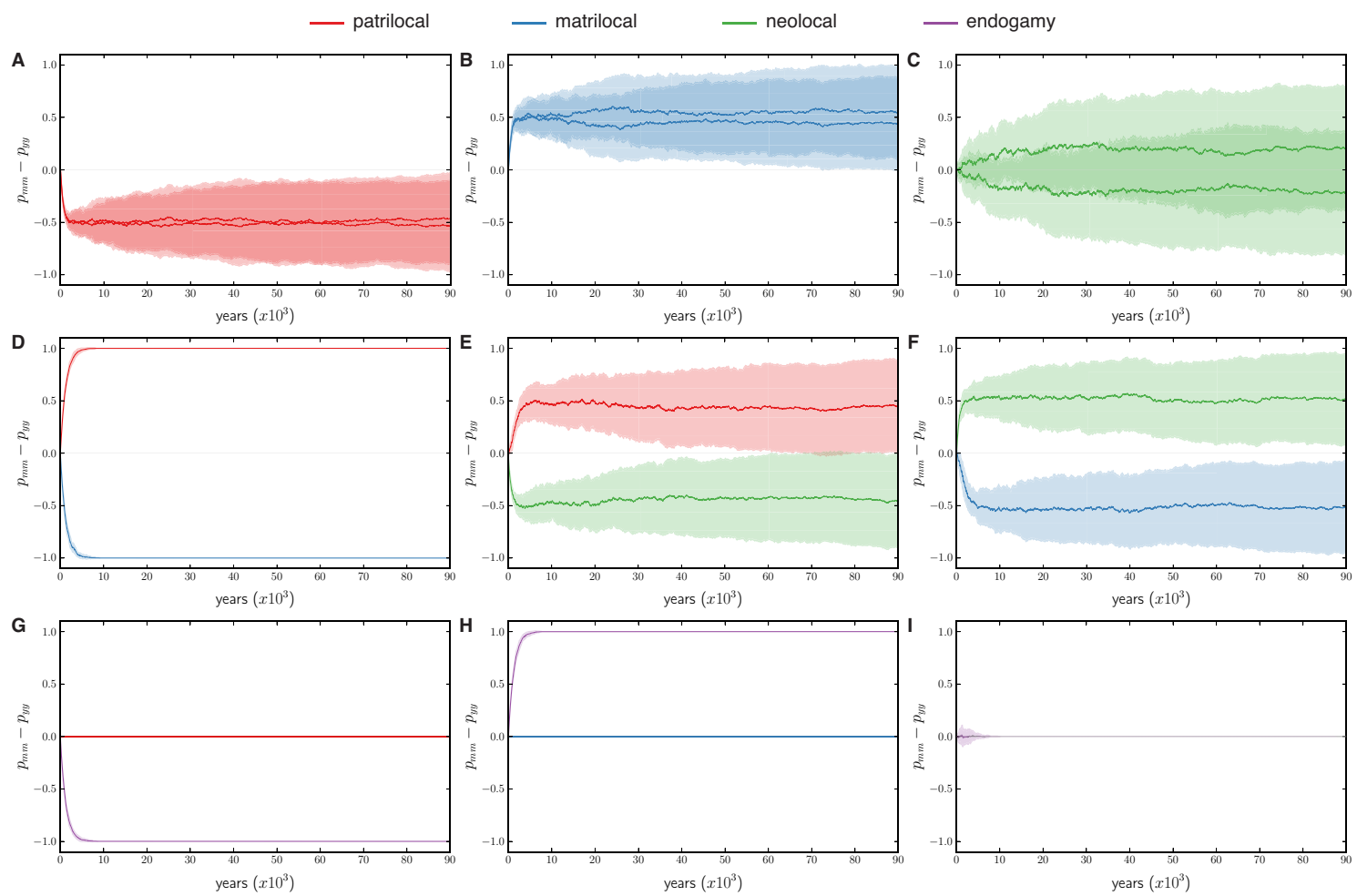

Figure 2.5: Two-deme simulation of kinship-structured migrations. (A) Patrilocalpatrilocal, (B) matrilocal-matrilocal and (C) neolocal-neolocal migrations show the expected $p_{m m}-p_{y y}$ signal. When the exchange of males or females is assymetric as in (D) patrilocal-matrilocal, (E) patrilocal-neolocal, (F) matrilocal-neolocal, (G) patrilocal-endogamy and $(\mathrm{H})$ matrilocal-endogamy, cross-dressing is observed. (I) Neolocal-endogamy interactions results to one homogeneous and one well mixed villages. The trends appear distinct by around generation 200 and are sustained in all generations.

practice. We show in Fig. $2.5 p_{m m}$ and $p_{y y}$ comparisons for the same deme-deme interactions illustrated in Fig. 2.4. We follow the probability of sharing mtDNA and Y chromosome for 90,000 years or 3,600 generations with 50 males and 50 females in each deme. The agent-based model is run for $2^{5}$ randomizations.

We find that a homogeneous uniparental kinship practice does result to the expected signal of higher relatedness along the father line in patrilocal villages $\left(p_{m m}-p_{y y}<0\right.$ in Fig. 2.5A) and greater relatedness along the mother line in matrilocal villages $\left(p_{m m}-p_{y y}>0\right.$ in Fig. 2.5B) for all generations. A population practicing neolocality wherein husbands and wives are exchanged in both directions can either show "patrilocality" or "matrilocality" depending on the genes of the 
individuals initially chosen to migrate, die and mate.

If the kinship practice of the demes are not the same, sustained cross-dressing of the villages is found in all generations. In Fig. 2.5D, we see the patrilocal village appearing as if it was "matrilocal" and the matrilocal village as if it was "patrilocal". Interaction of a patrilocal (Fig. 2.5E) and a matrilocal (Fig. 2.5F) village with a neolocal village also results to trans-locality where the gender that in reality stays in the village appears to be migrating out. The neolocal village appears to be either "patrilocal" or "matrilocal" depending on whether there is a net outflow of males or females. A matrilocal or patrilocal village assymetrically sending migrants to an endogamous village results to the endogamous village mimicking their signal (Fig. 2.5G-H) . An endogamous village, however, shows equal mixing of both mtDNA and Y chromosome when it interacts with a neolocal village (Fig. 2.5I) . For Fig. 2.5G-I, the non-endogamous villages maintain their original mtDNA and Y chromosome hence $p_{m m}-p_{y y}=0$.

\section{3 n-deme Model of Migration in a Structured Population}

The same dynamical rules can be extended to the general case of a population geographically structured to $n$ demes by kinship practice. Forward migration rate $m$, which is the number of individuals moving per time step, is 1.0 for females in patrilocal villages and males in matrilocal villages. Males in patrilocal villages and females in matrilocal villages never migrate out and as such $m=0.0$.

In Fig. 2.6A-B, we see that for a population network of 10 patrilocal villages, $p_{m m}-p_{y y}$ is still negative for all sub-populations while for a population network of 10 matrilocal villages, $p_{m m}-p_{y y}$ is still positive as in the two-deme case. This is true regardless of the number of demes $n$ for as long as the population is homogeneous in kinship practice. When half of the population is patrilocal while the other half is matrilocal (Fig. 2.6C), we find the general behavior of the patrilocal villages crossdressing as "matrilocal" and the "matrilocal villages appearing as "patrilocal".

Migration networks with majority of the villages being either patrilocal (Fig. 2.6D) or matrilocal (Fig. 2.6E) but still interacting with villages of the opposite kinship practice shows all sub-populations cross-dressing. When all of the types of post- 


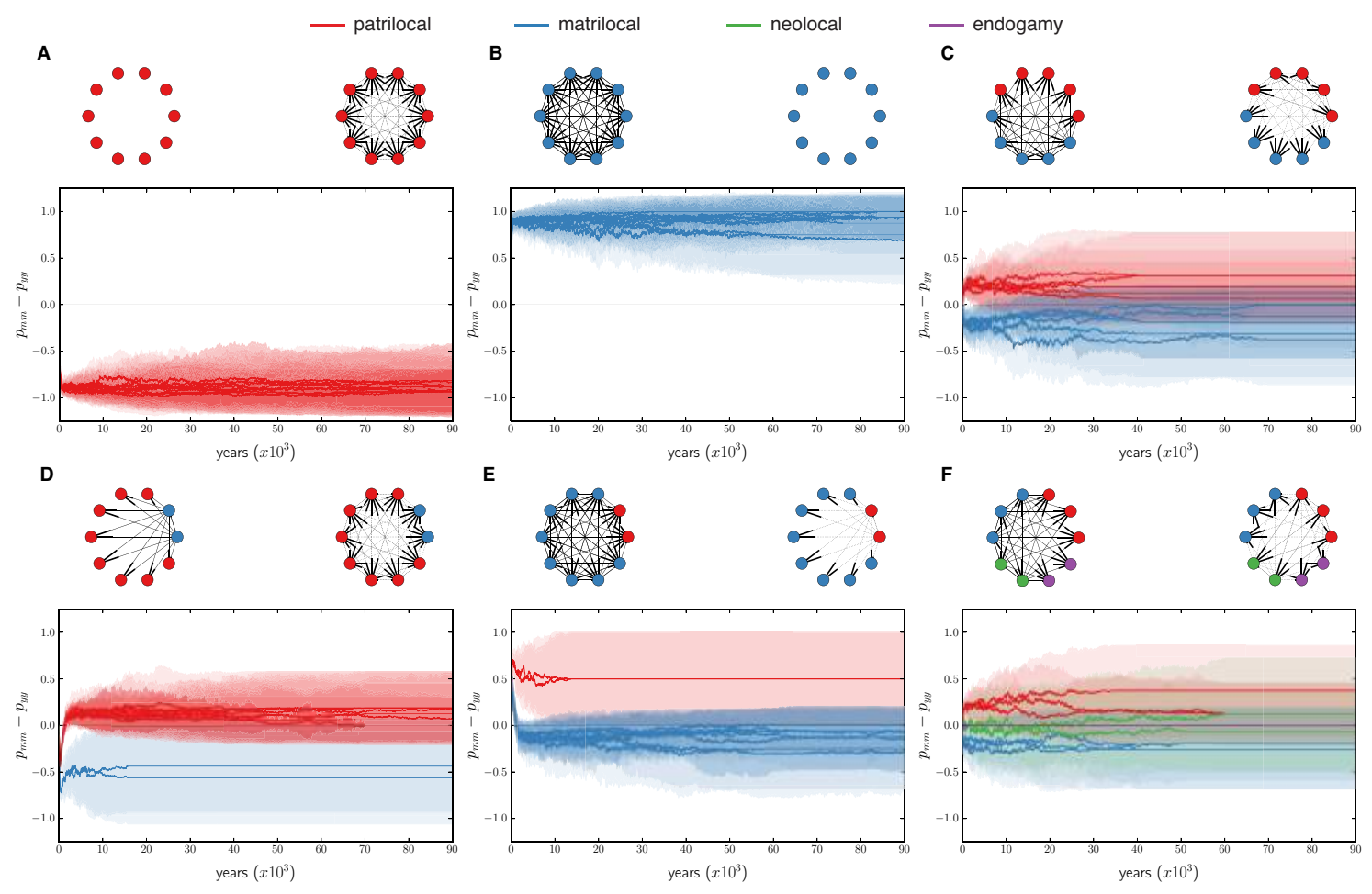

Figure 2.6: n-deme simulation of kinship-structured migrations. Comparison of mtDNA and Y chromosome similarities for a population divided into 10 demes by kinship-structured interactions: (A) all patrilocal, (B) all matrilocal, (C) 5 patrilocal and 5 matrilocal, (D) 8 patrilocal and 2 matrilocal, (E) 2 patrilocal and 8 matrilocal, and (F) 3 patrilocal, 3 matrilocal, 2 neolocal and 2 endogamous villages. The networks on the top left of the plots are the migration networks of males while those on their top right are the migration networks of females. Every link corresponds to an $m=1.0$. The mean $p_{m m}-p_{y y}$ is presented as solid lines together with the standard deviation of $2^{4}$ realizations. Cross-dressing is observed in (C)-(F) with the neolocal villages in (F) switching between being "patrilocal" and "matrilocal" in the course of the simulations. 
marital residence rules exist in the population as in Fig. $2.6 \mathrm{~F}$, the patrilocal and matrilocal villages switch signal and exhibits trans-locality. Cross-dressing for these villages is maintained throughout the simulations. However, the neolocal villages have a tendency to change dress and sometimes appear as "patrilocal" and sometimes as "matrilocal". Their signals are transient and stochastically depend on current flows of males and females. The endogamous villages remain equally mixed in mtDNA and Y chromosome when the amount of male and female immigrants are the same.

\subsection{Genetic Diversity in Sumba and Timor}

Kinship post-marital residence rules is strongly practiced in the Indonesian traditional societies of Sumba and Timor. Symmetric Prescriptive Alliance (APA) marriage rules and its influence on the genetic diversity of patrilocal clans in the Sumbanese village of Rindi has previously been studied [18]. However, as we've illustrated here, it is important to consider the interactions of the village with other villages to properly account for the observed genetic diversity. We use mtDNA and Y chromosome information collected by Lansing et al. [4, 16] from 14 matrilocal Sumbanese villages and 11 Timorese villages, two of which practices patrilocality and nine follows matrilocality (Fig. 1.2).

Using a bayesian coalescent approach, the mtDNA and Y chromosome genetic trees of all individuals in Sumba and Timor are constructed. Employing the same method, genetic trees were also constructed separately for each of the villlages. Additionally, to investigate the general effect of kinship practice when more than one post-marital residence rule exist, we also create a clustered matrilocal and patrilocal mtDNA and Y chromosome trees for Timor. The probability of sharing a common ancestor is calculated for each generation $g$ by finding if pairs of individuals have common ancestors at or earlier than $g$.

For Sumba where all villages are reported to practice matrilocality, we expect it to be more probable for individuals to share a common ancestor in the matriline than in the patriline. We see in Fig. 2.7A that this is true for all generations except for those of time depth $51-57$ kya. Dissecting the Sumba locality signal into the separate village signals, we find in Fig. 2.7B that all the matrilocal villages 
- all
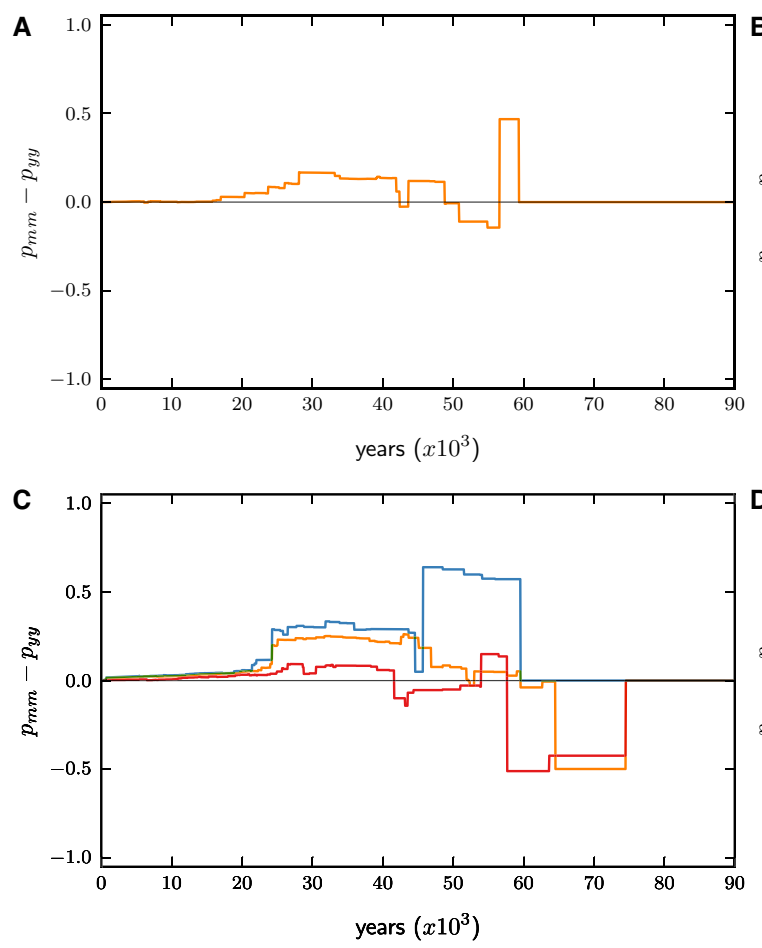

- patrilocal

- matrilocal
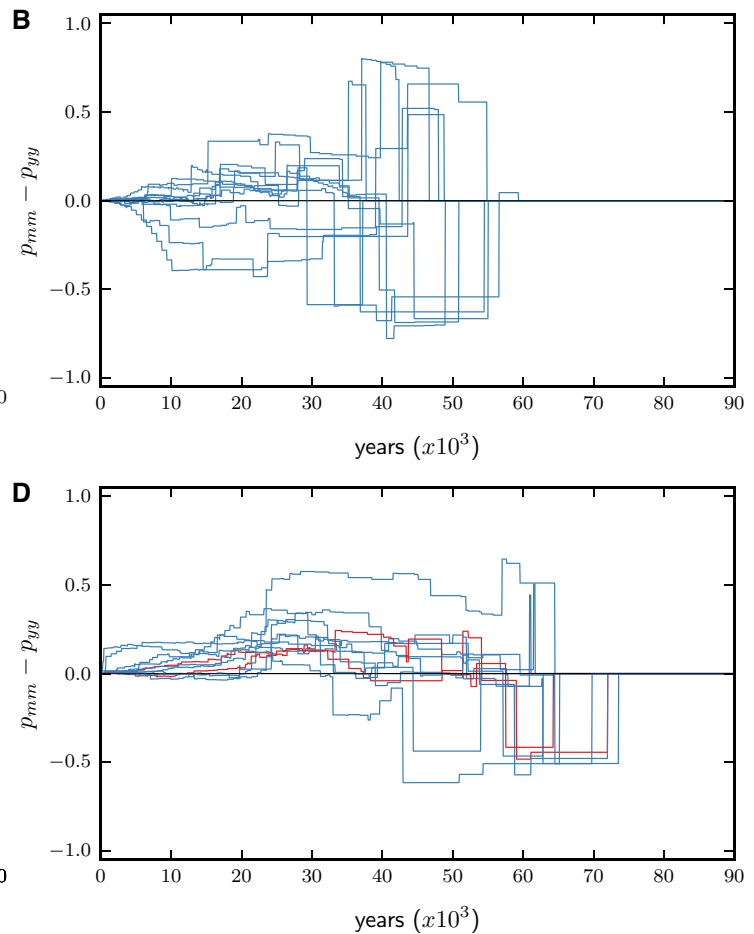

Figure 2.7: mtDNA and $Y$ chromosome diversity comparison in Sumba and Timor. $p_{m m}-p_{y y}$ is shown in the top row for the 12 matrilocal villages of Sumba and in the bottom row for the 2 patrilocal villages and 9 matrilocal villages of Timor. (A) Sharing of a common ancestor in the mtDNA and Y chromosome trees of all individuals in Sumba show "matrilocality" signal except for time depths $51-57$ kya. (B) Analyzing the villages separately show transient cross-dressings. (C) The probability comparison of sharing an ancestor in the mtDNA and Y chromosome trees of all, matrilocal and patrilocal individuals of Timor is shown. Patrilocal villages always cross-dress while matrilocal villages switch locality signal. (D) Timor patrilocal villages cross-dresses until 58 kya while matrilocal villages behave like neolocal villages with some showing "patrilocality" and some "matrilocality". 
transiently cross-dress with more trans-locality observed at the $51-57$ kya period. We've seen in Fig. 2.6F that such transient trans-locality or switching of kinship practice signal happens for neolocal villages interacting with matrilocal or patrilocal villages. This hints to the presence of some degree of neolocality wherein some females migrate out of some villages even if they are expected to reside in their natal village after marriage.

The influence of a mixture of kinship practices on genetic diversity can be further tested on Timor. The n-deme simulation (Fig. 2.6E) similar to the Timor case shows sustained cross-dressing of all villages. The Timor sample, however, shows that the clustered matrilocal villages (Fig. 2.7C) do not cross-dress while patrilocal villages switches locality signal several times. Looking at the kinship practice locality signal separately for each of the villages (Fig. 2.7D), we find that for the patrilocal villages, trans-locality is observed for generations less than $60 k y$ old. This suggests three possibilities, (1) adherence to patrilocality is not strictly followed, (2) their deep ancestral structure used to be matrilocal, or (3) they interacted with only patrilocal villages in the deep past. Several transient switching is observed for the Timorese matrilocal villages similar to the Sumbanese matrilocal villages. Except for two matrilocal villages that have slightly negative locality signal in the recent past, they show a locality signal of their actual kinship practice, that is $p_{m m}-p_{y y}>0$ for matrilocality, up until 30 kya. This suggests that either (1) they have little to no interaction with the patrilocal villages, or (2) they don't strictly adhere to matrilocality. The signal in the deep past of the Timorese matrilocal villages suggest that some degree of neolocality or relaxation of kinship practice was previously present.

\subsection{Adherence to Kinship Practice}

We can further explore the hypothesis that the deviations from the model of our data is a result of relaxed kinship practice and/or limited exogamous interactions of the villages. The kinship systems practiced in Sumba and Timor which up to now holds as a result of their isolation from the rest of the world are indicated in Figs. 1.2 and 1.3. For this purpose, we consider an additional assumption that all migration events are for mating purposes. This requires accounting for gender imbalance in the 

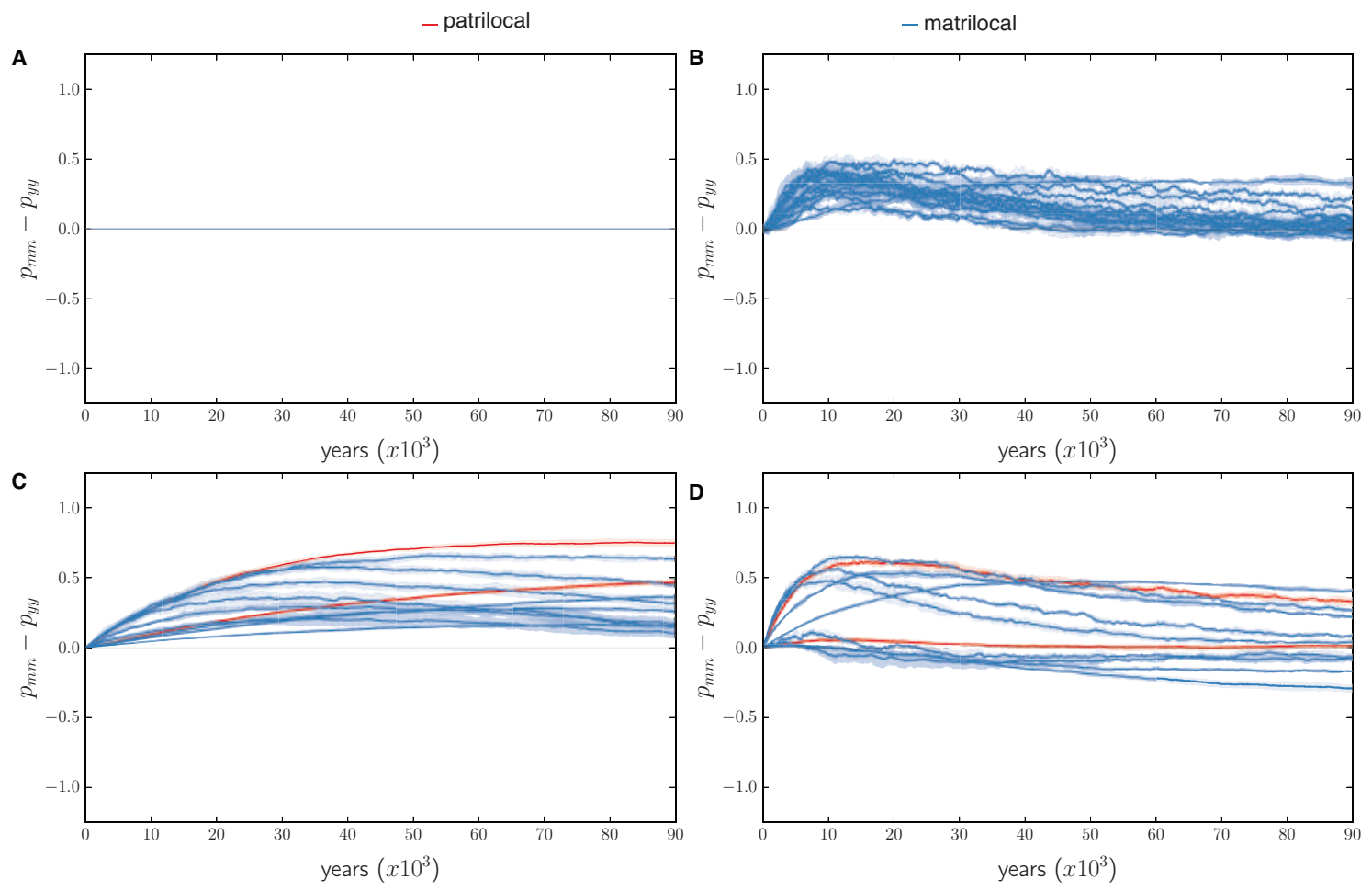

Figure 2.8: Effects of relaxed kinship practice and limited interactions to joint genetic diversity (A) Matrilocal Sumbanese villages under strict kinship practice shows similar mtDNA and Y chromosome diversity due to its net female village populations. (B) Dissimilar genetic diversities and cross-dressing for the villages initialized to be Sumbanese happens when kinship practice is relaxed and dispersals are limited. (C) For Timorese villages, only patrilocal villages cross-dress under strict kinship practice. (D) Relaxing rules and limiting interactions result to transient cross-dressings for all Timorese villages.

villages. A male can only migrate to vilages with a net female population $\left(N_{m}<N_{f}\right)$ while a female can only migrate to villages with a net male population $\left(N_{m}>N_{f}\right)$. Similarly, individuals looking for a mate will prefer to find one within their natal village. They only disperse and migrate out when no husband/wife is available because of gender balance being against them. In a previous study of Lasing et al. the male and female effective population sizes for the Sumbanese and Timorese villages considered here were calculated [36]. We use this to initialize the number of males and females in the villages of our population.

With the post-marital residence rules fully implemented, we see in Fig. 2.8A that matrilocal Sumbanese villages show no difference in the probability of sharing 
a common ancestor in their matrilines and patrilines. This results from the villages having net female populations. As such, there are more than enough available wives in the natal villages of men that there's no sufficient incentive for them to migrate out. However, it may happen that kinship practice is relaxed such that females also migrate out. For the case of Sumba, this is compelling as a gender imbalance of more females means that not all females will find a husband within the village. In Fig. 2.8B, we see that when kinship rules are relaxed and females migrate out at a rate of 0.05 and males have a limited dispersal of rate 0.10 , transient cross-dressing of the villages is observed similar to what is found in the Sumba data.

When kinship practices are mixed such as in the case of Timor, strict adherence to kinship practices result to only the patrilocal villages exhibiting trans-locality (Fig. 2.8C). This is explained by the fact that the two patrilocal villages are overpowered by the nine matrilocal villages causing them to receive more male immigrants. On the other hand, the female immigrants of the matrilocal villages is not as significant. But when we limit dispersals of women in patrilocal villages and men in matrilocal villages to a rate of 0.5 , and relax kinship practice by allowing men to migrate out of patrilocal villages and women to migrate out of matrilocal villages both at a rate of 0.05 , transient cross-dressing of all villages regardless of their kinship practice is observed (Fig. 2.8D).

\subsection{Summary and Conclusions}

Migrations in traditional societies like Sumba and Timor are mostly motivated by marriage and the union of resources. Kinship practices that define post-marital residence rules of the villages structure the way individuals move in the population. Individuals in endogamous villages prefer to marry within the existing clans of their village. Neolocality allows anyone regardless of gender to migrate out of their natal villages. Patrilocality and matrilocality have gender biased movements such that females frequently migrate out of patrilocal villages whole males often choose to reside outside of their natal matrilocal village.

Uninformed hypothesis might lead us to expect that individuals from matrilocal villages will be more related in the matriline since females stay after marriage while individuals from patrilocal villages will have closer kins in the patriline since males 
reside in their natal village after marriage. However, genetic data from the Indonesian islands of Sumba and Timor tells us otherwise. And this is because villages cannot be considered in isolation. They exist within the context of a network of interacting villages, each possibly having a distinct kinship practice.

Hence, we observe the existence of the phenomena of trans-locality wherein the villages appear to cross-dress. That is, a village that in reality practices matrilocality shows a signal of "patrilocality" in that it appears to be more related in the patriline. Similarly, a patrilocal village may show a signal of "matrilocality" and appear more related in the matriline. This happens when (1) a mixture of different types of kinship practices exists in the population, (2) interaction of villages are limited such as when migration rates are low or (3) kinship rules are not strictly followed.

\subsection{Future Work}

In this study, we considered a fully connected network of villages, as a possible extension, other types of village migration interactions can be investigated. What if there is a village that acts as the center of the region and as such attracts more migrants as compared to other villages? What if there is a preferential attachment in the way villages interact? What if there is a socio-economic inequality among villages in the region? How can this be quantified and consequently, how can this affect the movement of migrants?

In the model implemented here, we also considered a constant village size. In reality, populations within villages may expand or contract. We suggest adding a model of population growth into the agent-based model presented here. One can also investigate variations on this: (1) cases wherein the population growth of males and females differ within villages, and (2) cases wherein population growth differs between villages.

A more specialized extension that can be done together with a geneticist is employing a mating mechanism into the agent-based model. Such that the genetic composition of offsprings are not merely just a direct copy of the parents' Y chromosome and mtDNA, but instead results from genetic processes involving mutations and recombinations. 


\section{Chapter 3}

\section{Kinship Channels of Language and Genes}

Language moves down time in a current of its own making, as Edward Sapir famously observed [37]. Here we suggest that these currents flow in channels created by kinship systems $[38,39,40]$. To discover how durable such channels are, and how long languages remain within them, we investigated language transmission in 25 communities on the remote eastern Indonesian islands of Sumba and Timor, where language diversity and traditional settlement structures are still largely intact [41]. In these traditional societies, movements among communities are mostly motivated by marriage $[42,43,44]$. Broadly, there are four possibilities: i) all individuals marry and reside within the same community (endogamy); ii) individuals marry and live outside their natal community (ambilocality or neolocality); iii) men remain in their natal community and women disperse (patrilocality); or iv) women remain in their natal community and men disperse (matrilocality) [45]. In tribal societies like Sumba and Timor where language diversity is high, these dispersal practices have different consequences $[46,47,48,49]$. When women remain in their natal communities and men disperse (matrilocality), language transmission is channeled through females and children will learn the community language of their mothers. In this case, if men often marry outside the radius of their mothers speech community, language might be expected to correlate with the maternally inherited mitochondrial DNA (mtDNA), but not with the paternally inherited Y chromosome (Y). Conversely, if men stay in their natal community and women disperse (patrilocality), the opposite pattern should hold [50]. 
To examine these mechanisms of language transmission, we analyzed linguistic and genetic data from two Indonesian islands where postmarital residence rules are still largely observed and dispersals are mostly local. These communities mimic how the rest of the world once looked, and provide a model system to investigate language transmission without many of the repercussions of globalization that affect the modern world. On Sumba, we surveyed 14 patrilocal villages where 12 Austronesian languages are spoken (Fig. 1.3). Many communities on neighboring Timor are also patrilocal, but there is also a centuries-old cluster of matrilocal villages in the region of Wehali near the center of the island [51]. We surveyed nine matrilocal villages in Wehali, along with two patrilocal villages located just to the north. The languages spoken in these villages include a non-Austronesian language (Bunak) and four Austronesian languages. Thus, our study system includes 25 villages on two islands, with roughly equal numbers of matrilocal and patrilocal communities, speaking 17 languages belonging to two language families. How have these languages traveled through time, within and between communities?

\subsection{Association between genes and languages}

To find out, we began by collecting data on language and kinship from representative samples of men in each village. Our goal was to compare matrilineal and patrilineal pathways of language transmission for each individual. Although matrilineal descent can be inferred for both men and women from their maternally inherited mtDNA, patrilineal descent must be traced with the $\mathrm{Y}$ chromosome, carried only by men. Therefore, only males were sampled for this study. We recorded the languages spoken by each individual, as well as genealogical records (including birthplace) extending back to great-grandparents. The goal was to discover which ancestors were the source of the language(s) each individual learned as a child. Genetic distances were inferred between individuals on both mtDNA and $Y$, and this information was used to trace genealogical relationships much deeper into the past. 


\subsubsection{Methods of shared gene-language heritage}

\subsubsection{Language Tree}

The linguistic materials were organized and classified according to the principles of traditional comparative method. The resulting language phylogenies for Sumba and Timor were validated using classifications provided by Ethnologue [?]. These language phylogenies were then calibrated using penalized likelihood and a relaxed substitution rate model for inferring internal branching times [?]. The final language tree of Sumba (Fig. 3.1A) was constructed by setting the root to 4,085 , the year the Austronesian speakers arrived in Sumba as dated by $\mathrm{Xu}$ et al. [?]. The final language tree shown of Timor (Fig. 3.1B) was constructed with two priors: (1) the Timorese Austronesian languages were set to split at 5,000 years ago; and (2) the contemporary languages on Timor were assumed to have a coalescent date no earlier than 10,000 years ago.

\subsubsection{Probability of shared gene-language heritage}

The probability that a pair of individuals $(i, j)$ share a common language $l$ given that they belong to the same genetic clade $g$ at generation $t$ is given by

$$
P\left(i_{l}, j_{l} \mid i_{g(t)}, j_{g(t)}\right)=\frac{P\left(i_{l}, j_{l} \cap i_{g(t)}, j_{g(t)}\right)}{P\left(i_{g(t)}, j_{g(t)}\right)}=\frac{\sum_{g=1}^{c_{t}}\left(\sum_{i, j \neq i} 1_{\cos \left(\mathbf{1}_{i_{g}}, \mathbf{l}_{j_{g}}\right)>0}\right)}{\sum_{g=1}^{c_{t}} n_{g}\left(n_{g}-1\right) / 2},
$$

where $c_{t}$ is the number of genetic clades in generation $t$ each lasting 25 years, $\mathbf{l}_{i_{g}}$ and $\mathbf{l}_{j_{g}}$ are the language vectors of individuals $i$ and $j$ belonging to $g, 1_{\cos \left(\mathbf{1}_{i_{g}}, \mathbf{l}_{j_{g}}\right)>0}$ is 1 if $i$ and $j$ share a language and 0 otherwise, and $n_{g}$ are the number of individuals in $g$.

\subsubsection{Results of shared gene-language heritage}

The resulting associations between groups of related men on Sumba and Timor and the languages they speak are shown in Fig. 4.2. Matrilineal relatedness is shown on the lefthand panels (Fig. 4.2A,C) and patrilineal relatedness on the righthand panels (Fig. 4.2B,D). The color bands at the bottom of each phylogeny indicate the languages spoken by each individual, including multiple colors if the individual 


\section{A Sumba}

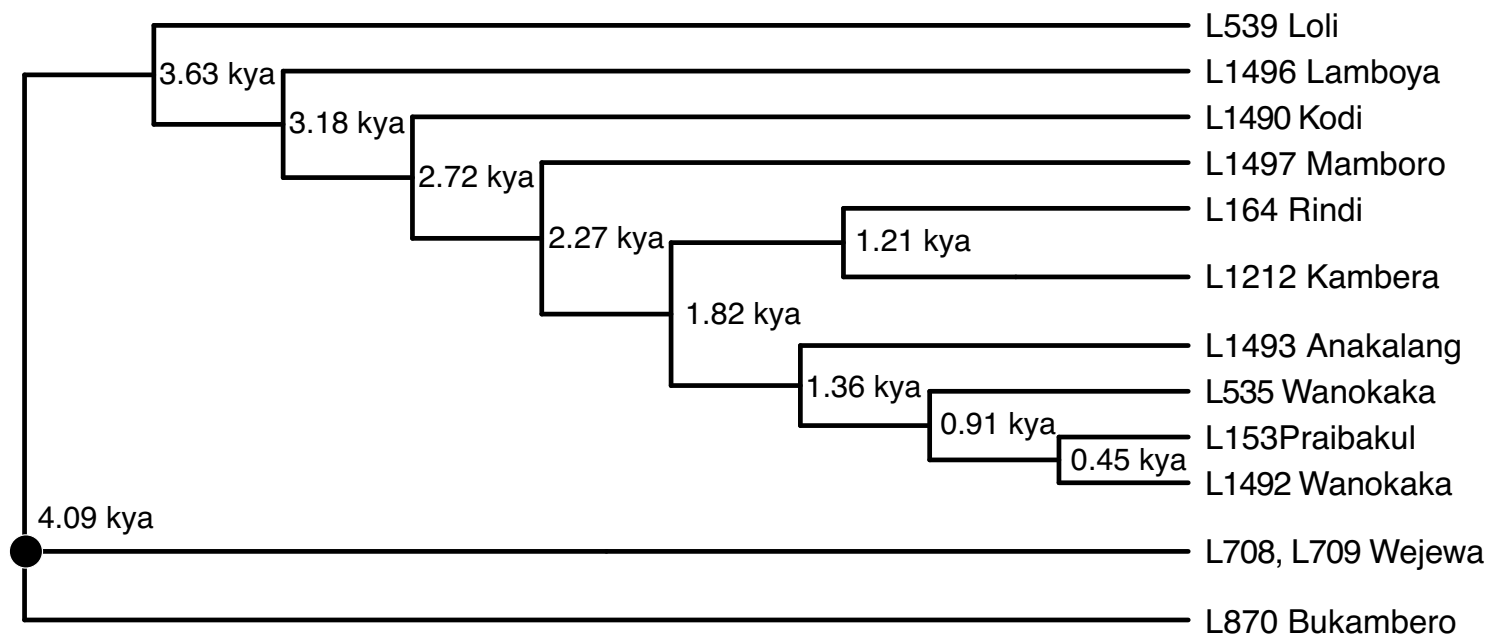

\section{B Timor}

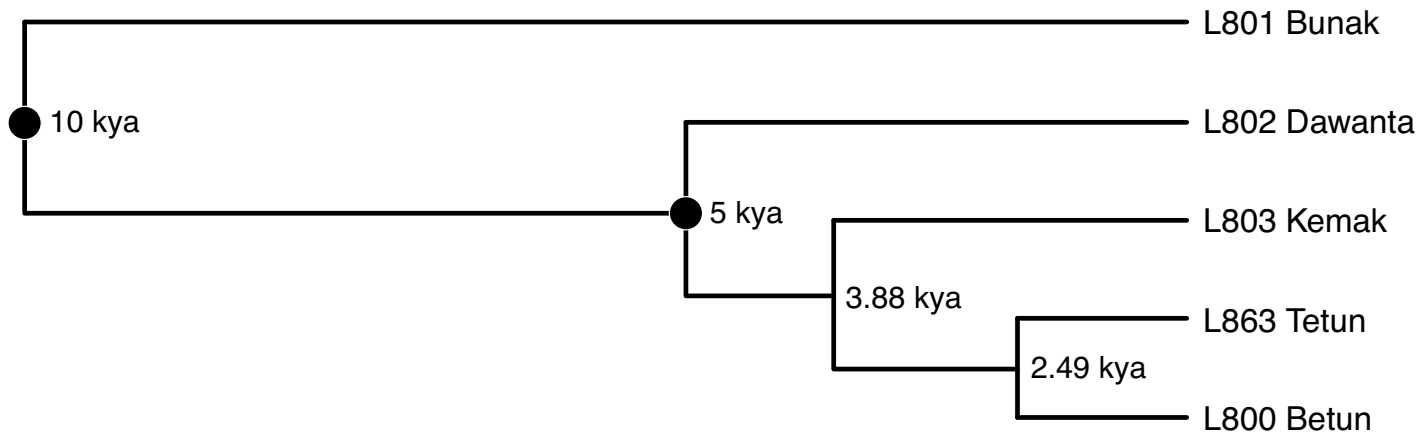

Figure 3.1: Language Tree. The root of the (A) Sumba language tree is set at 4.085 kya. In (B) Timor, the Papuan language Bunak splits off at 10 kya and the Timorese Austronesian languages at 5 kya. These calibration points are shown as $\bullet$

is bi- or multilingual. The thickness of each segment in the color band indicates the size of groups of individuals who speak a common language and are are closely related genetically. Both the Y chromosome tree for patrilocal Sumba communities (Fig. 4.2B) and the mtDNA tree for matrilocal Timor communities (Fig. 4.2C) contain larger clades of related individuals who speak a common language (25 and 47 individuals in the largest clades of Sumba $\mathrm{Y}$ and Timor mtDNA), compared to the trees of the dispersing sex (12 and 26 individuals in the largest clades of Sumba mtDNA and Timor Y). Larger groups of individuals who have close genetic relationships and speak a common language are therefore found in the lineages of the non-dispersing sex. 

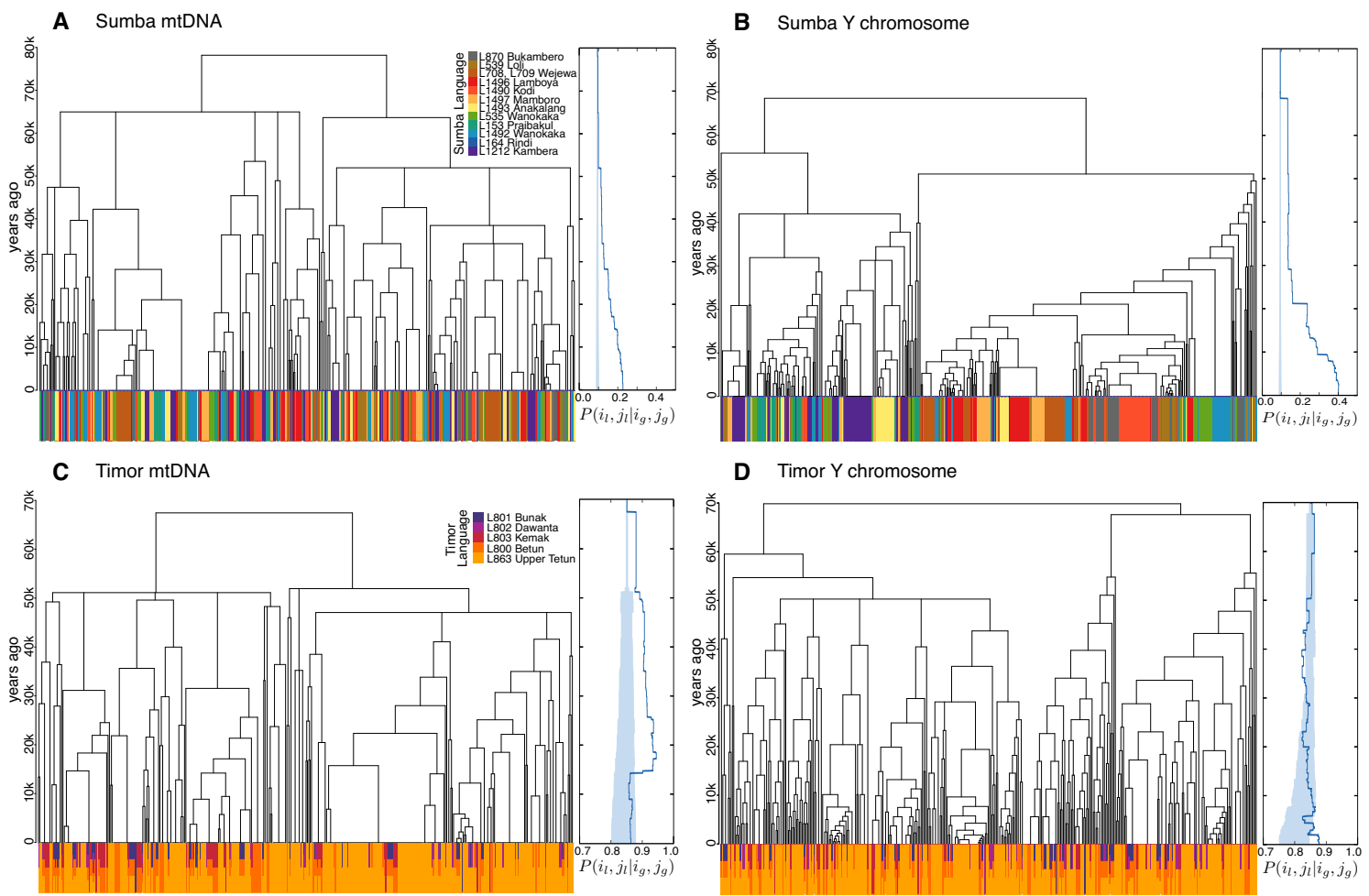

Figure 3.2: Language sharing in the mtDNA and Y phylogenies of (A-B) Sumba and (C-D) Timor. Color bands beneath the phylogenies show the languages spoken by each individual (monolingual in Sumba; sometimes multilingual in Timor). Plots to the right of each phylogeny show the probability of sharing a language $l$ given that each pair of individuals are in the same genetic clade $g$ at a given time in the past. Solid lines represent the observed metric, with shaded bands indicating the results of random permutations of the linguistic data. Higher probabilities that close genetic relatives share a language, compared with random expectations, are observed for (B) Sumba Y and (C) Timor mtDNA at all time periods. For (A) Sumba mtDNA and (D) Timor Y, probabilities are only higher than random cases until $\sim 65,000$ and $\sim 1,500$ years ago, respectively. 
The hypothesis that kinship practices create durable channels for language transmission was tested by comparing the topologies of the mtDNA and Y chromosome trees. We surmise that the statistical significance of the different-sized groups of closely related individuals speaking a common language reflects the influence of community structure on language transmission. Specifically, they represent persistent speech communities created by the vertical transmission of languages along genetic clades, as mediated by kinship rules. To test this hypothesis, we define a probability $P\left(i_{l}, j_{l} \mid i_{g(t)}, j_{g(t)}\right)$ that a pair of individuals $(i, j)$ share a common language $l$, given that they belong to the same genetic clade $g$ at some time in the past $t$. This metric indicates greater than expected language sharing, even at decreasing degrees of genetic relatedness backwards in time.

We show this in the plots at the right of the trees in Fig. 4.2, with solid lines representing the observed data, and shaded regions indicating the range of probabilities seen when languages are shuffled randomly among samples.

As we track back through time (i.e., along branches in the tree), men who are related on their fathers' side in the patrilocal villages of Sumba are consistently more likely to speak a common language compared to random cases. This tendency is stronger along patrilines (Fig. 4.2B) than matrilines (Fig. 4.2A). Conversely, in mostly matrilocal Timor, men are more likely to share a common language with their close matrilineal kin (Fig. 4.2C-D).

We find evidence for the persistence of speech communities, where probabilities of sharing a common language with close kin are stronger and mostly distinguishable from random chance, notably for the Y chromosome in patrilocal Sumba and mtDNA in mostly matrilocal Timor. These speech communities appear to have formed as genes and languages followed the non-dispersing sex - speech communities on the father's side in patrilocal Sumba and the mother's side in matrilocal Timor. These results suggest two further hypotheses, which we explore below:

1. Kinship rules concerning marriage and postmarital residence can persist for many generations and predict population genetic structure at the community scale; and

2. The association between genetic and language clades, as created by kinship structures, provides information not only about language transmission, but also about the structure and persistence of social groups. 
These arguments turn on the answers to two questions. First, how do kinship rules relate to population genetic structure? And second, what is the relationship between the channels created by kinship practices and the transmission of languages? How long do such channels persist, and when do languages shift between them? We begin with a simple model to explore these scenarios, and then compare the results to the empirical data.

\subsection{Kinship and population genetic structure}

We begin by exploring how kinship rules affect the movements of men and women between villages. For this purpose we adapted an Isolation with Migration (IM) coalescent model [52] to capture the genetic consequences of different female and male migration patterns. This can be used to directly assess evidence for postmarital residence practice: i) village endogamy, ii) ambi- or neolocality, iii) patrilocality, and iv) matrilocality.

\subsubsection{Kinship Isolation with Migration (IM) model}

We used the Isolation with Migration model to capture the impact of sex-biased movements on genetic diversity [52]. Using scaled (haploid) migration rate $M=$ $2 N m$ and $\theta=2 N \mu$, with mutation rate $\mu$ in mutations per generation in the locus, the probability that the number of nucleotide differences between a pair of individuals $S_{j}$ is $k$ is

$$
\begin{aligned}
P\left(S_{j}=k\right)= & \sum_{r=1}^{2} A_{j r}\left(\frac{\lambda_{r} \theta^{k}}{\left(\lambda_{r}+\theta\right)^{k+1}}\left(1-e^{-\tau\left(\lambda_{r}+\theta\right)} \sum_{l=0}^{k} \frac{\left(\lambda_{r}+\theta\right)^{l} \tau^{l}}{l !}\right)\right. \\
& \left.+\frac{e^{-\tau\left(\lambda_{r}+\theta\right)}(a \theta)^{k}}{(1+a \theta)^{k+1}} \sum_{l=0}^{k} \frac{\left(\frac{1}{a}+\theta\right)^{l} \tau^{l}}{l !}\right)
\end{aligned}
$$

where $j=0$ and $j=1$ correspond to the cases of two samples being from the same or different villages respectively, and

$$
\begin{aligned}
& \lambda_{1}=\frac{n M+n-1-\sqrt{D}}{2(n-1)} \\
& \lambda_{2}=\frac{n M+n-1+\sqrt{D}}{2(n-1)},
\end{aligned}
$$


and

$$
D=(n M+n-1)^{2}-4(n-1) M,
$$

and

$$
\begin{aligned}
& A_{01}=\frac{\lambda_{2}-1}{\lambda_{2}-\lambda_{1}} \\
& A_{02}=\frac{1-\lambda_{1}}{\lambda_{2}-\lambda_{1}} \\
& A_{11}=\frac{\lambda_{2}}{\lambda_{2}-\lambda_{1}} \\
& A_{02}=\frac{-\lambda_{1}}{\lambda_{2}-\lambda_{1}} .
\end{aligned}
$$

We calculated these equations twice, once with $M=M_{\text {male }}=2 N m_{\text {male }}$ and once with $M=M_{\text {female }}=2 N m_{\text {female }}$. When fitting the model, we sample from the four distributions $P\left(S_{j}=k\right)$ according to the number of pairwise comparisons in the data that are within or between villages.

This model is a considerable simplification of the complex migration patterns generated by kinship systems. It assumes (1) independence of the movement of mtDNA and Y chromosomes; (2) that the effective population size $N$ and demographic history, as described by $n, \tau$ and $a$, of the mtDNA and Y chromosome are the same (in practice, $N$ is often lower for men due to higher reproductive variance); and (3) standard coalescent assumptions, such as a relatively small sample size compared to population size and exchangeability among females and males. A further simplification in this model is the assumption that a measurement of pairwise differences between two individuals in a sample is independent of the number of pairwise differences between other individuals in the sample.

Nevertheless, this non-equilibrium model can incorporate demographic events that are expected to be reflected in the data (e.g. a population expansion associated with the Out of Africa migration). It is able to closely fit the distribution of pairwise differences for distant relatives observed in the Sumba and Timor mtDNA and Y chromosome data (see main text Fig. 3) as well as differences between these loci at smaller genetic distances. We are especially interested in the fitting at smaller genetic distances as these better reflect the impact of kinship system on recent genetic history. While we do not believe that fitted parameters map directly to corresponding real-world properties of these populations, they can capture biases 
that give a qualitative indication of whether matrilocality or patrilocality is reflected in our data.

\subsubsection{Fitting the kinship IM model using Approximate Bayesian Com- putation $(\mathrm{ABC})$}

$\mathrm{ABC}$ is a method to assess how much the observed data supports different parameter values in a model. We first scale the pairwise Bruvo's distances [30] between Y chromosomes (values in range $[0,1]$ ) such that the average observed distance is the same as for the mtDNA pairwise differences. Given that the time depth of the $\mathrm{Y}$ chromosome and mtDNA trees are similar we consider this operation appropriate in 'scaling' the mutation rate of the $\mathrm{Y}$ chromosome and mtDNA such that, for the purposes of modeling, they can be considered equal $\left(\mu=1.67 \mathrm{e}^{-7} * 25 * 540=\right.$ 0.002214). Having obtained the observed distributions, we proceed with the ABC iterations.

In the simple form of $\mathrm{ABC}$ used, an iteration consists of (1) proposing a combination of parameter values according to the parameter prior distributions (all with uniform priors with bounds: $50 \leqslant N \leqslant 500,2 \leqslant n \leqslant 200,0.0 \leqslant m_{\text {male }} \leqslant 1.0$, $0.0 \leqslant m_{\text {female }} \leqslant 1.0,0.1 \leqslant \tau \leqslant 10,0.01 \leqslant a \leqslant 2.0 ;(2)$ calculating the probability distribution of pairwise distances that the parameter values imply, for within and between village comparisons of both mtDNA and Y chromosome; (3) multinomial sampling from these distributions according to the number of within- and between-village pairs in the data; and (4) calculating the distance (KullbackLeibler divergence was used) of the model from the observed distributions of pairwise differences. We iterate through this procedure two billion times, keeping the parameters generating the model output with the lowest distance from the data as representative of the posterior distributions. The small bounds on $a$ were chosen after observing the rejection of large $a$ in a less restrictive fitting $(0.01 \leqslant a \leqslant 1000)$. The ABC posterior distributions of the IM model (in main text Fig. 3) are shown in Figs. 3.3 and Figs. 3.4. The prior distribution of $T_{0}$ and Ancestral $N$ are calculated from the uniform priors of other parameters. 


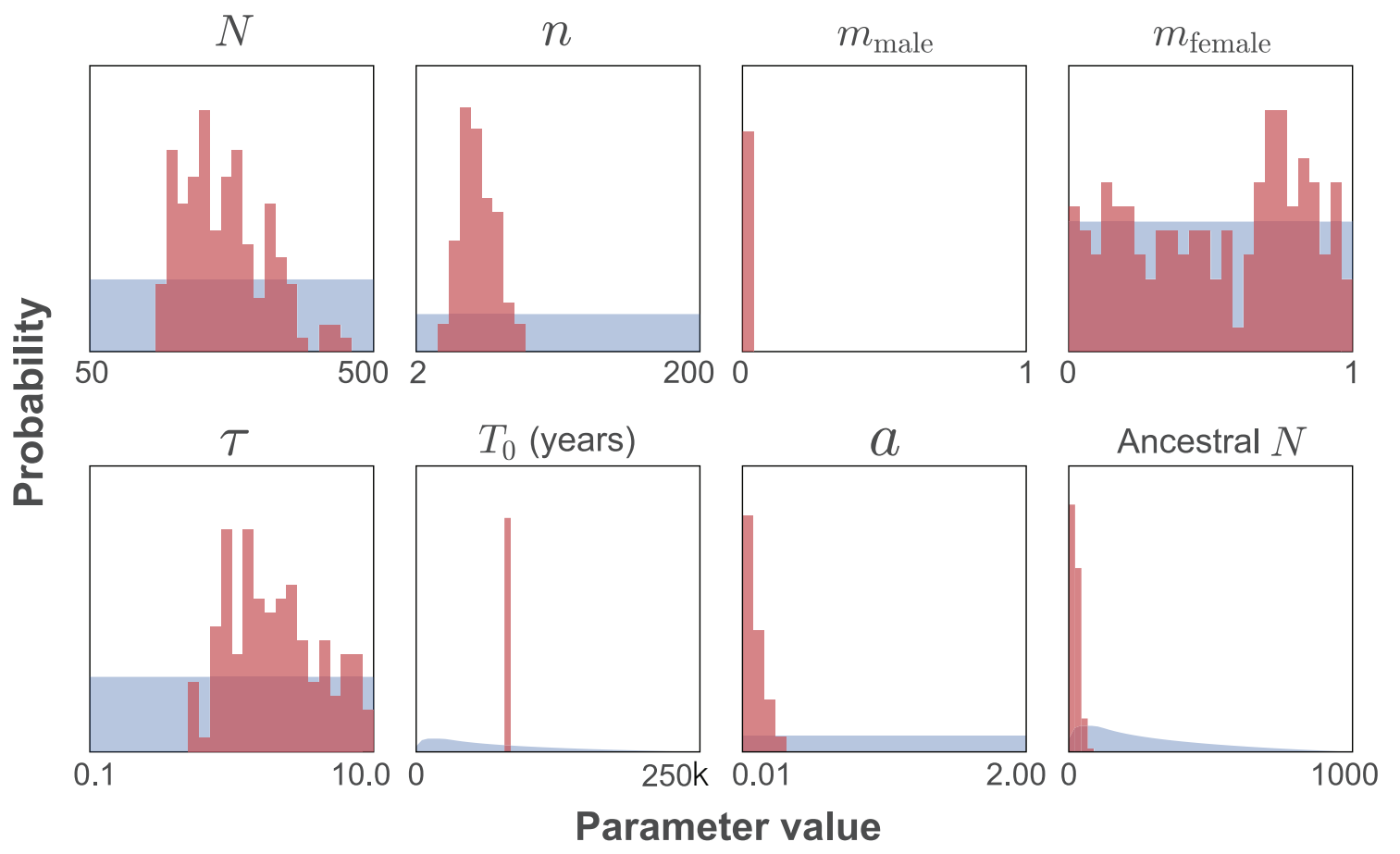

Figure 3.3: Posterior distributions of IM model fitting for Sumba data, based on best-fitting 150 parameter values of approximately 2 bn samples from prior distributions as indicated.

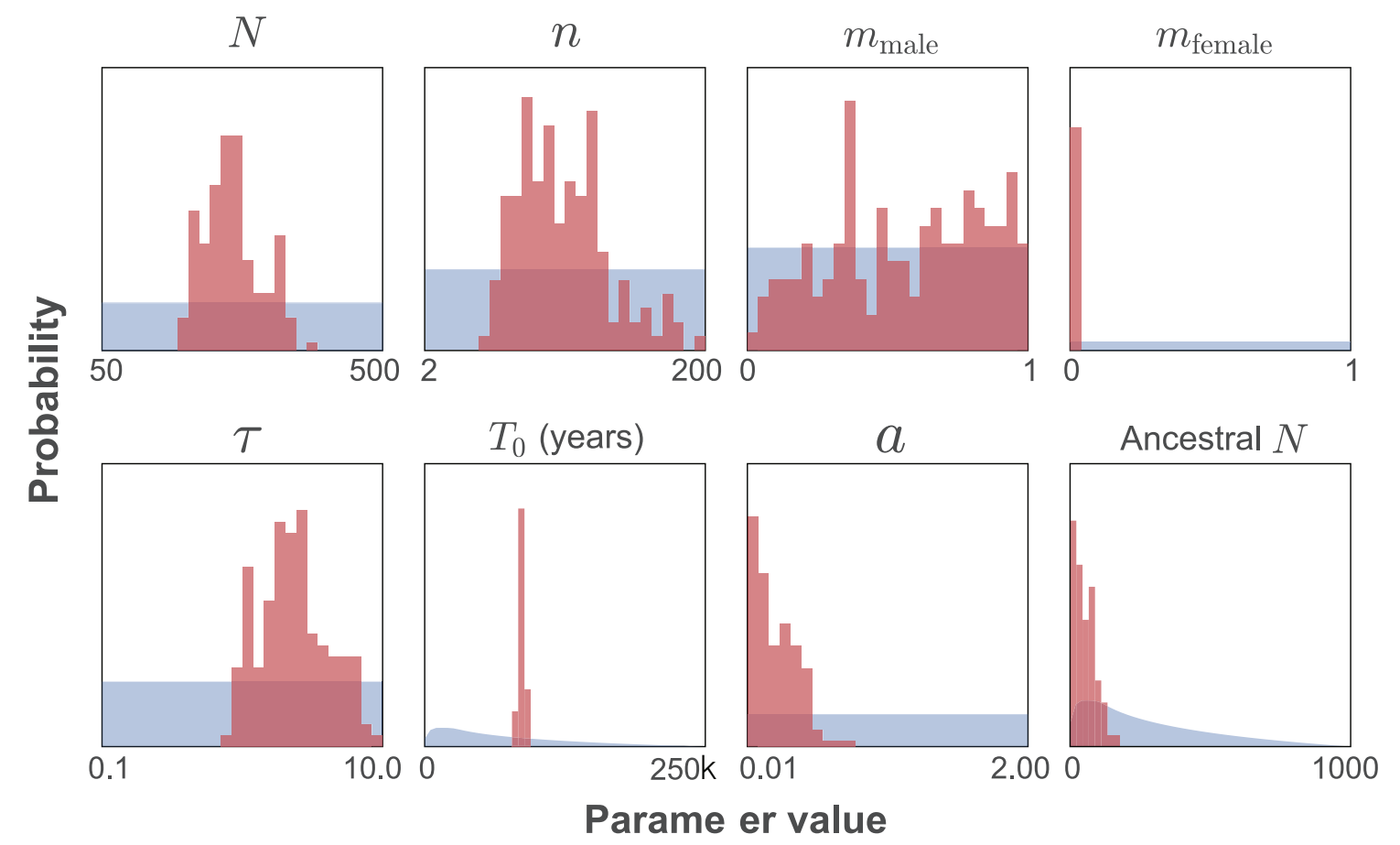

Figure 3.4: Posterior distributions of IM model fitting for Timor data, based on bestfitting 150 parameter values of approximately 2 bn samples from prior distributions as indicated. 


\subsubsection{Parameter space of the kinship IM model within and between villages}

The mtDNA and Y chromosome pairwise distances within and between sub-populations are shown in Fig. 3.5. The KL divergence for between village comparisons Fig. 3.5B is almost the same for all migration rate values. Differences in the model fit as quantified by the KL divergence only become apparent when looking at within village comparisons Fig. 3.5A. It is only easy to observe kinship systems through differences in mtDNA and Y chromosome pairwise distances within subpopulations. This is an important future consideration when designing methods to detect the signature of kinship practices.

\subsubsection{Results of kinship and population genetic structure}

The IM model describes a single panmictic population of size $a N$ splitting into $n$ subpopulations of size $N$ at time $2 N \tau$ generations in the past. Migration occurs at a rate $m$ between subpopulations. To assess the effects of postmarital residence practices on mtDNA and Y chromosome, we distinguish between the migration rates of women (mtDNA) and men (Y), and run the model separately for each group. Both runs have the same values of $N, n, a, \tau$ and mutation rate $\mu$, but can have different migration rates $m_{\text {female }}$ and $m_{\text {male }}$. For example, matrilocal kinship systems lead to greater migration of men, such that $m_{\text {male }}>m_{\text {female. }}$ The converse is true for patrilocal systems. A cultural preference for endogamy is reflected by low migration rates for both $m_{\text {female }}$ and $m_{\text {male }}$.

Fig. 3.6A-D shows typical outputs from this model in the form of paired genetic distances. Here each individual is paired with every other individual, and the pairs are represented by single points corresponding to how closely they are related to one another on both mtDNA (matrilineal) and Y (patrilineal). In the simplest cases (endogamy, and ambi- or neolocality), there is no bias towards matri- or patrilineal relatedness (Fig. 3.6A-B). Consequently, given equal mutation rates, the distribution of pairwise mtDNA and $\mathrm{Y}$ distances lies on the one-to-one correlation line, at a distance from origin that depends on demographic parameters. If both females and males marry and reside within their natal villages (Fig. 3.6A), we see two clusters of pairwise genetic distances: i) near the origin, a cluster of closely related kin 


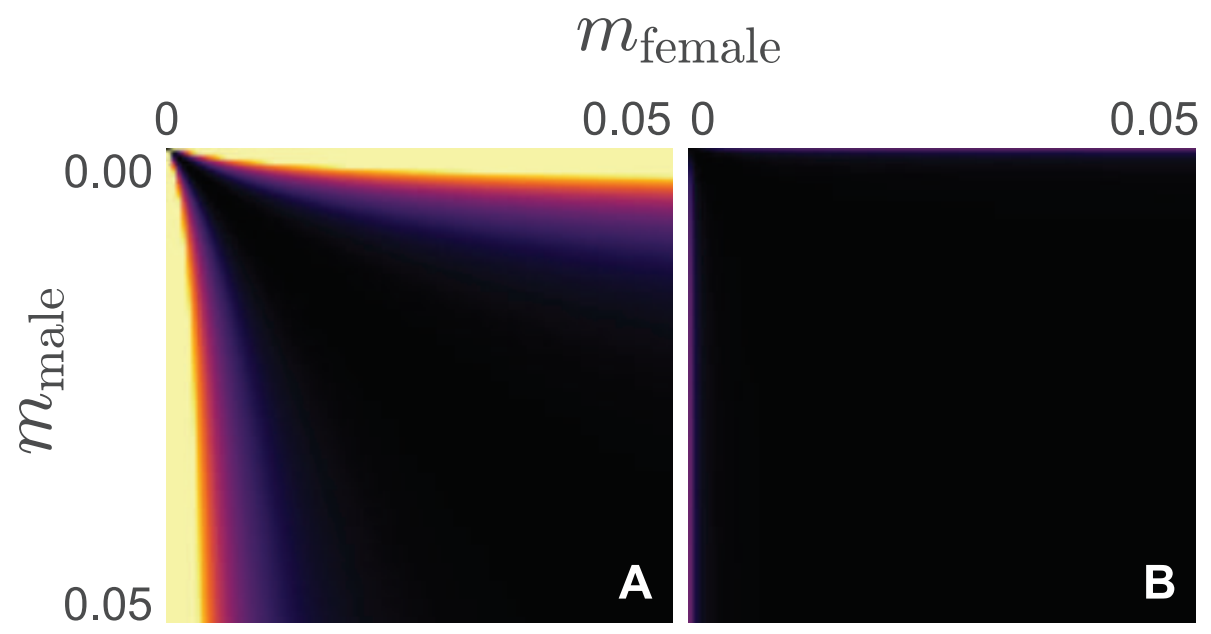

C
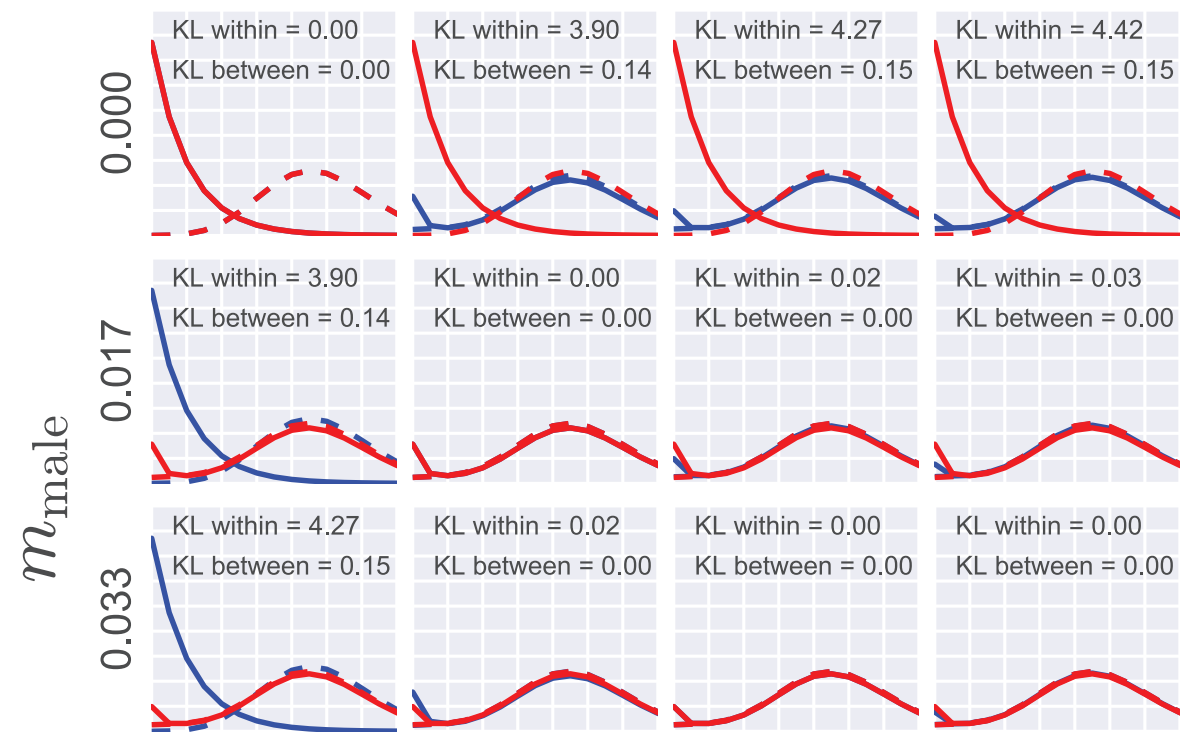

$\mathrm{KL}$ within $=0.00$

$\mathrm{KL}$ within $=0.02$

$\mathrm{KL}$ within $=0.03$

$\mathrm{KL}$ between $=0.00$

$\mathrm{KL}$ between $=0.00$

$\mathrm{KL}$ between $=0.00$
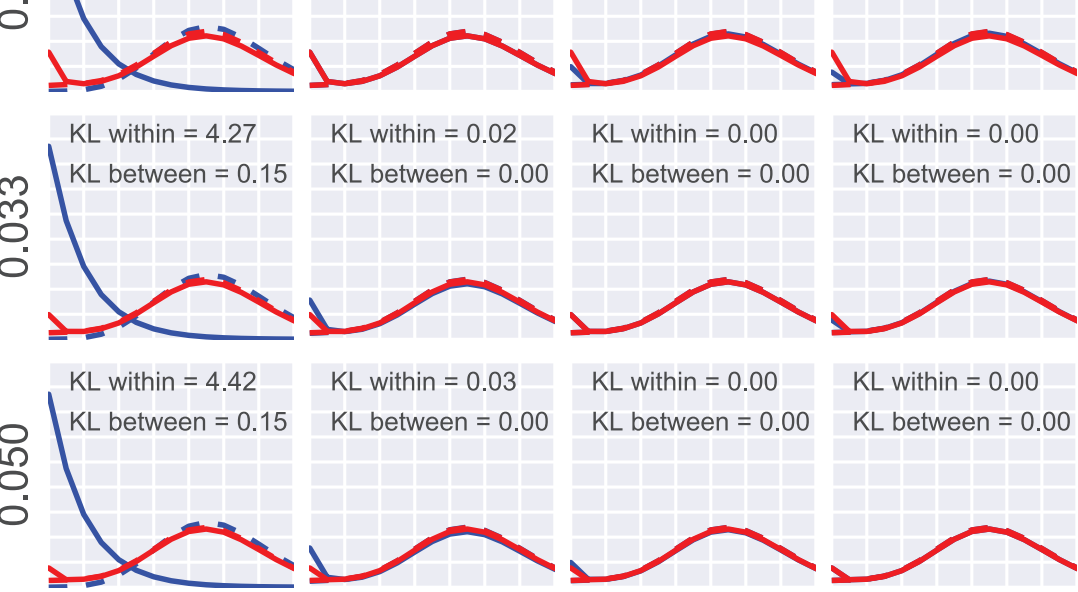

$\mathrm{KL}$ within $=0.02$

$\mathrm{KL}$ within $=0.00$

$\mathrm{KL}$ within $=0.00$

$\mathrm{KL}$ between $=0.00$

$\mathrm{KL}$ between $=0.00$

$\mathrm{KL}$ between $=0.00$
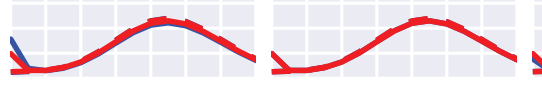

$\mathrm{KL}$ within $=0.03$

$\mathrm{KL}$ within $=0.00$

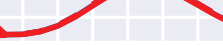

0.000
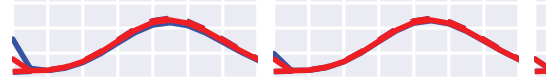

$\mathrm{KL}$ within $=0.00$

$\mathrm{KL}$ between $=0.00$

$\mathrm{KL}$ between $=0.00$

$\mathrm{KL}$ between $=0.00$

0.017

0.033

0.050

$m_{\text {female }}$

Figure 3.5: Migration rates phase space. (A) KL divergence of pairwise distance distributions $(\mathrm{KL}(\mathrm{Y}-\mathrm{mtDNA})+\mathrm{KL}(\operatorname{mtDNA}-\mathrm{Y}))$ within subpopulations. (B) KL divergence of pairwise distance distributions between subpopulations. (C) Example plots of mtDNA (blue lines) and Y chromosome (red lines) pairwise distance distributions within (solid line) and between (dashed line) subpopulations, focusing on the low-difference regime. 

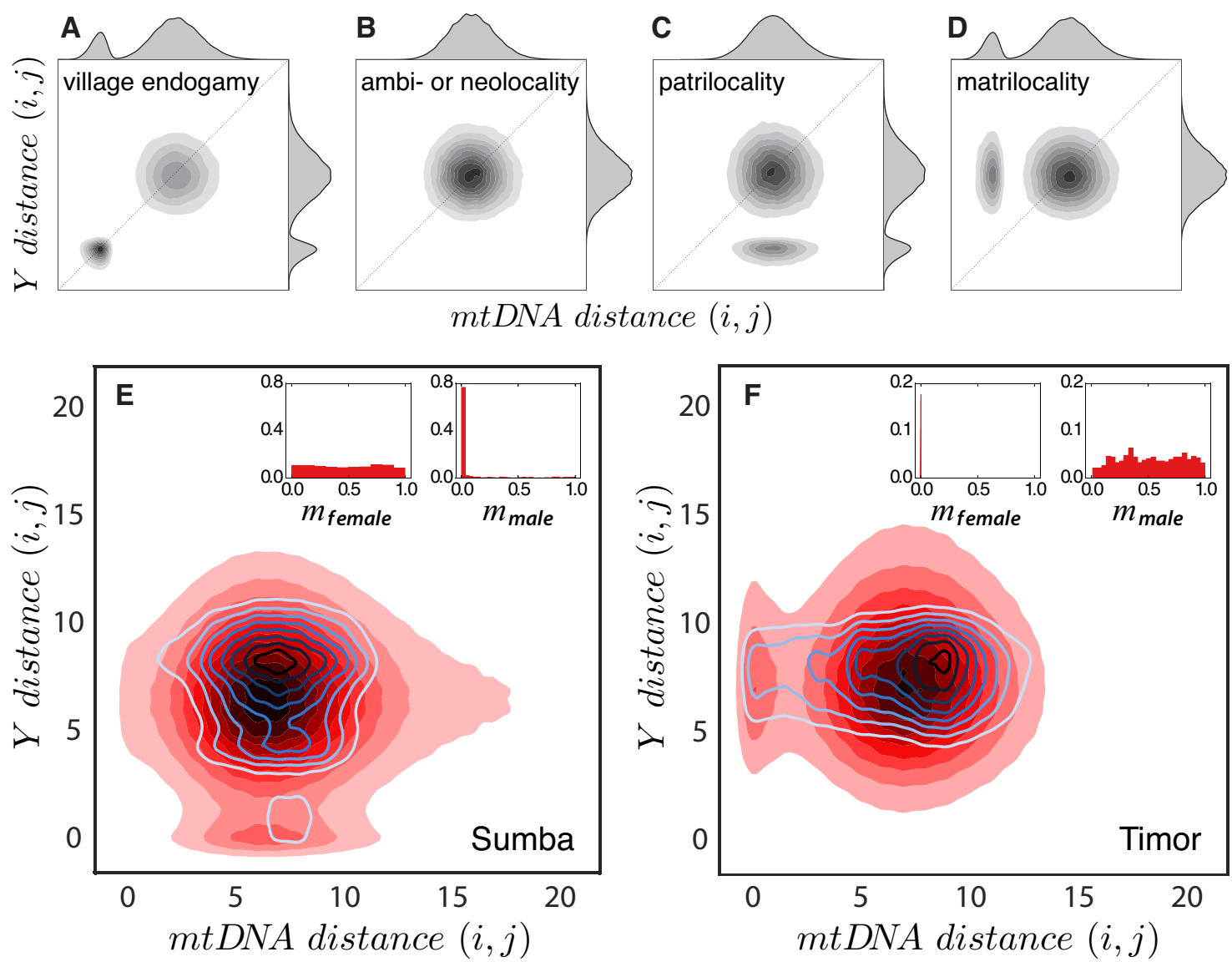

Figure 3.6: Genetic structure from an IM model with migration influenced by kinship practices. To identify the theoretical role of kinship practices on genetic diversity, the IM model was run for a population that are (A) endogamous, (B) ambi- or neolocal, (C) patrilocal and (D) matrilocal. (E) Close correspondence of the IM model (red shading) with observed data (blue contours) for patrilocal Sumba using $N=298, n=41, m_{\text {female }}=0.73, m_{\text {male }}=9 x 10^{-5}, \tau=5.49$ and $a=0.024$. (F) Close correspondence of the IM model with matrilocal Timor using $N=264$, $n=97, m_{\text {female }}=1 x 10^{-5}, m_{\text {male }}=0.25, \tau=6.71$ and $a=0.06$. The insets (E) and $(\mathrm{F})$ show the posterior distributions of migration rates for the kinship system in Sumba and Timor based on 3 billion samples drawn from prior distributions of all IM model parameters. 
residing in the same village, and ii) a larger cluster consisting of individuals living in different villages, and thus less closely related. When there is no gender bias in dispersal and both sexes move frequently, the distributions of genetic distances for mtDNA and Y are similar (Fig. 3.6B). Introducing a matri- or patrilocal bias in marriage customs shifts the pairwise distance plot towards one or the other axis (Fig. 3.6C-D). In patrilocal villages, where men remain in their natal villages while women move to marry, pairs of men remain closely related on their Y, but not their mtDNA (Fig. 3.6C). The opposite holds for matrilocal villages where women remain in their natal villages and men move after marrying (Fig. 3.6D).

These predictions are borne out in the genetic data from Sumba (Fig. 3.6E) and Timor (Fig. 3.6F). By plotting the pairwise genetic distance, we can see how closely individuals are related on their mtDNA (matriline) and Y chromosome (patriline). In Sumba, the cluster of small Y distances (close patrilineal kin) is more distinct comprising $11 \%$ of all pairs, as compared to the faint cluster of small mtDNA distances (close matrilineal kin) with only $5.1 \%$ of pairs. On the other hand, in Timor, the more pronounced cluster is of small mtDNA distances comprising $8.3 \%$ of all pairs, in contrast to the cluster of small $\mathrm{Y}$ distances with $7.6 \%$ of pairs. Comparing the two islands (with more detail shown in Fig. 3.9A-B), there is a clear tendency for closer patrilineal relatedness in Sumba, while in Timor we see the opposite pattern of closer matrilineal relatedness.

How much time is needed for these patterns to emerge, assuming a constant sex bias in migration rates? To find out, we used Approximate Bayesian Computation (ABC) rejection sampling to assess which IM model parameters closely match the data. This method returns the posterior distribution of female and male migration rates that best fit the observed data (Fig. 3.6E-F insets). The migration rate of the rarely dispersing sex is tightly constrained, while the migration rates of the other sex are not.

\subsubsection{Alternative isolation with migration model}

We propose below a more realistic alternative to the kinship isolation with migration model that reflects the two stages of migrations in Sumba and Timor. However, we do not present here how it performs in fitting the actual data as it is computationally more intensive because of the number of fitting parameters and we find that results 
of the above presented kinship IM model sufficiently captures the structure of our data. However, future works may benefit from the model we present below.

To investigate how speech communities persists, we look at how movements of both men and women among villages are structured by the kinship system. Extending the Isolation with Migration model $[53,54,55,56]$ to describe dispersals in the communities, we study how (1) neolocality/endogamy, (2) matrilocality and (3) patrilocality affect the genetic composition of the population.

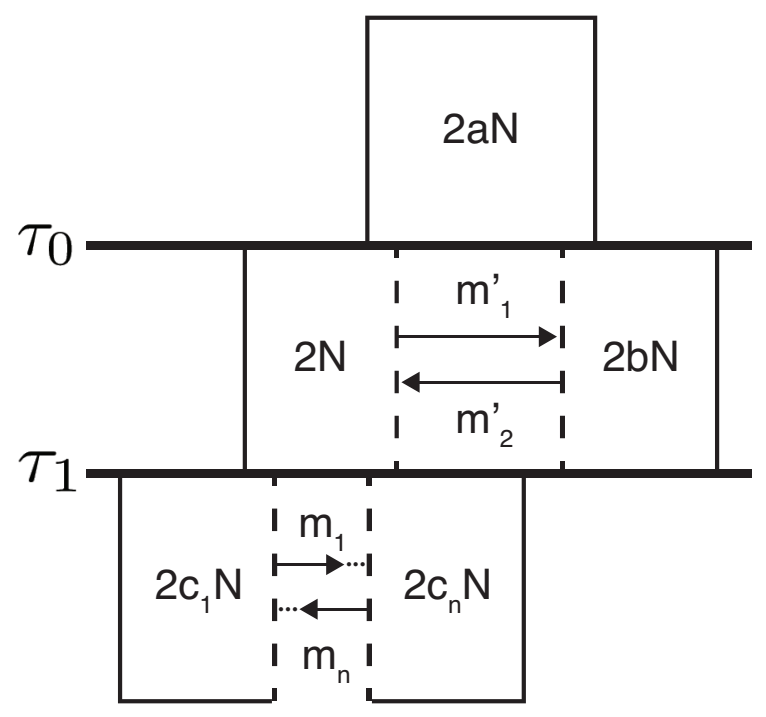

Figure 3.7: Two-stage isolation with migration model. First isolation with migration occurs between two sub-populations at time $T_{0}$. Sub-population 1 with size $2 N$ further divides into $n$ sub-populations at time $T_{1}$.

This coalescent model shown in Fig. 3.7 describes a single panmictic population of size $2 a N$ splitting into 2 subpopulations of size $2 N$ and $2 b N$ at a time $2 N \tau_{0}$ generations in the past. This mimics the contact of boat-travelling Austronesians and local Papuans in the islands of Indonesia. Migration occurs as a rate $m_{1}^{\prime}$ and $m_{2}^{\prime}$ between the subpopulations. At time $2 N \tau_{1}$ generations, the $2 N$ sub-population further divides into $n$ islands of size $2 c_{i} N$ each. Migrations among them happen at rate $m_{i}$. We use the scaled migration rates $M^{\prime}=2 N m^{\prime}$ and $M=2 N m$.

For the ancestral population before time $T_{0}=2 N \tau_{0}$ generations, the transition matrix for the not coalesced (1) and coalesced (0) states is given by 
(1) (0)

$$
\mathbf{Q}_{\text {anc }}=\underset{(0)}{(1)}\left(\begin{array}{cc}
-\frac{1}{a} & \frac{1}{a} \\
0 & 0
\end{array}\right)
$$

The two sub-populations at time between $T_{0}=2 N \tau_{0}$ and $T_{1}=2 N \tau_{1}$ generations follow the structured coalescence for a finite-sized population with a transition matrix

(1)

$$
\mathbf{Q}_{\text {mig }^{\prime}}=\begin{gathered}
(1) \\
(3) \\
(2) \\
(0)
\end{gathered}\left(\begin{array}{cccc}
-\left(1+M_{1}^{\prime}\right) & M_{1}^{\prime} & 0 & 1 \\
\frac{M_{2}^{\prime}}{2} & \frac{M 1_{1}+M_{2}^{\prime}}{2} & \frac{M_{1}^{\prime}}{2} & 0 \\
0 & M_{2}^{\prime} & -\left(\frac{1}{b}+M_{2}^{\prime}\right) & \frac{1}{b} \\
0 & 0 & 0 & 0
\end{array}\right)
$$

of states when (1) the pair of individuals are both in sub-population 1, (2) the pair of individuals are both in sub-population 2, (3) one is in sub-population 1 and another in 2, and (0) the pair has coalesced. The $n$ sub-populations after $T_{1}$ when the Austronesian contact in the islands of Indonesia happened interact with a transition matrix of

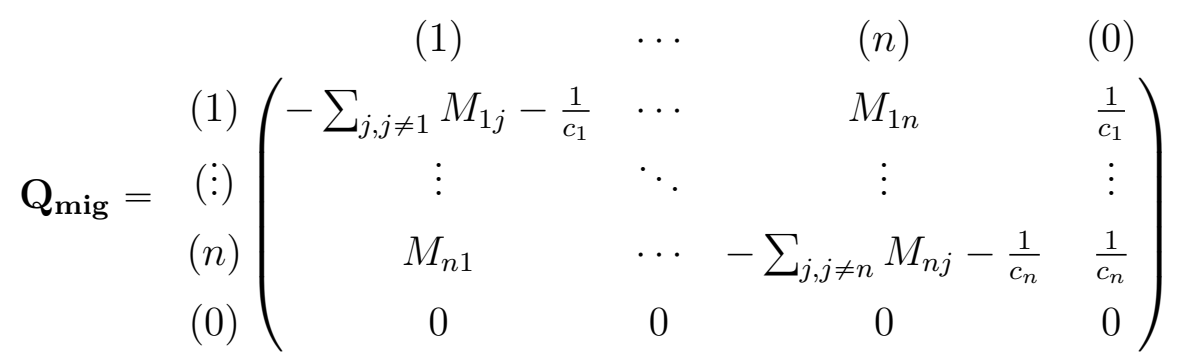

The probability distribution of pairwise coalescence times given that the process starts in state $i$ is thus given by

$$
f_{T}^{(i)}(t)=\left\{\begin{array}{lr}
f_{m i g}^{(i)}(t), & \text { for } 0 \leqslant t \leqslant \tau_{1} \\
{\left[1-F_{m i g}^{(i)}\left(\tau_{1}\right)\right] f_{m i g^{\prime}}^{(i)}\left(t-\tau_{1}\right),} & \text { for } 0<t \leqslant \tau_{0} \\
{\left[1-F_{m i g}^{(i)}\left(\tau_{1}\right)\right]\left[1-F_{m i g^{\prime}}^{(i)}\left(\tau_{0}-\tau_{1}\right)\right] f_{\text {anc }}^{(1)}\left(t-\tau_{0}\right),} & \text { for } \tau_{0}<t<\infty \\
0, & \text { otherwise }
\end{array}\right.
$$


where $F_{m i g}$ and $F_{m i g^{\prime}}$ are the cumulative distributions corresponding to migrations $M$ and $M^{\prime}$ respectively.

If $\mathbf{G}, \mathbf{C}$ and $\mathbf{D}$ are the right eigenvector matrices of $\mathbf{Q}_{\mathbf{m i g}}, \mathbf{Q}_{\mathbf{m i g}}$ and $\mathbf{Q}_{\mathbf{a n c}}$ respectively, and $-\alpha,-\beta$ and $-\gamma$ are the negative real eigenvalues then with mutation rate $\mu$ being mutation per generation in the locus, the probability that the number of nucleotide differences between a pair of individuals $S_{i}$ is $s$ is

$$
\begin{aligned}
P\left(S_{i}=s\right)= & -\sum_{j=1}^{n} G_{i j}^{-1} G_{j 0} \frac{\alpha_{j} \theta^{s}}{\left(\alpha_{j}+\theta\right)^{s+1}}\left\{1-e^{-\left(\theta+\alpha_{j}\right) \tau_{1}} \sum_{l=0}^{s} \frac{\left(\alpha_{j}+\theta\right)^{l} \tau_{1}^{l}}{l !}\right\} \\
& -\sum_{j=0}^{n} p_{i j}^{(1)}\left(\tau_{1}\right) \sum_{k=1}^{n} C_{1 k}^{-1} C_{k 0} \frac{\beta_{k} \theta^{s}}{\left(\beta_{k}+\theta\right)^{s+1}} \\
& \left\{e^{-\theta \tau_{1}} \sum_{l=0}^{s} \frac{\left(\beta_{k}+\theta\right)^{l} \tau_{1}^{l}}{l !}-e^{-\theta \tau_{0}-\beta_{k}\left(\tau_{0}-\tau_{1}\right)} \sum_{l=0}^{s} \frac{\left(\beta_{k}+\theta\right)^{l} \tau_{0}^{l}}{l !}\right\} \\
& -\sum_{j=0}^{n} p_{i j}^{(1)}\left(\tau_{1}\right)-\sum_{k=0}^{3} p_{j k}^{(2)}\left(\tau_{0}-\tau_{1}\right) \sum_{h=1}^{n} D_{k h}^{-1} D_{h 0} \frac{\gamma_{h} \theta^{s}}{\left(\gamma_{h}+\theta\right)^{s+1}} \\
& e^{-\theta \tau_{0}} \sum_{l=0}^{s} \frac{\left(\gamma_{h}+\theta\right)^{l} \tau_{0}^{l}}{l !}
\end{aligned}
$$

where $p_{i j}^{(1)}(t)=\sum_{k=0}^{n} G_{i k}^{-1} G_{k j} e^{-\alpha_{k} t}$ and $p_{i j}^{(2)}(t)=\sum_{k=0}^{3} C_{i k}^{-1} C_{k j} e^{-\beta_{k} t}$.

To capture the impact of kinship systems on the Y-chromosome and mtDNA, this model is run twice. Both runs should have the same values of $N, n, a, b, c, \tau_{1}$ and $\tau_{0}$, but should be allowed to have different migration rates $m_{\mathrm{mtDNA}}$ and $m_{\mathrm{Y}}$. For example, a matrilocal kinship system leads to faster movement of men such that $m_{\mathrm{Y}}>m_{\mathrm{mtDNA}}$; the converse is true for a patrilocal system. A general preference to exogamy corresponds to higher values of $m_{\text {mtDNA }}$ and $m_{\mathrm{Y}}$, while endogamy would be reflected by lower values.

If we let $N=500, n=10, a=0.75, b=0.25, c_{i}=1.0, \tau_{1}=190$ and $\tau_{0}=200$ (corresponding to 5000 years with a generation of 25 years), we can investigate the mtDNA and Y chromosome joint genetic differences density for the different kinship practices. The migration rates $m^{\prime}$ are set at 0.5 for both directions of mtDNA and $\mathrm{Y}$ chromosome movements. The migration rates $\left(m_{\mathrm{mtDNA}}\right.$ and $\left.m_{\mathrm{Y}}\right)$ in the $\mathrm{n}$ islands stage is given as $(0.0,0.0)$ for endogamy, $(1.0,1.0)$ for neolocal, $(1.0,0.0)$ for patrilocal and $(0.0,1.0)$ for matrilocal. This joint density of mtDNA and $\mathrm{Y}$ chromosome pairwise genetic distances is shown in Fig. 3.8. 
A

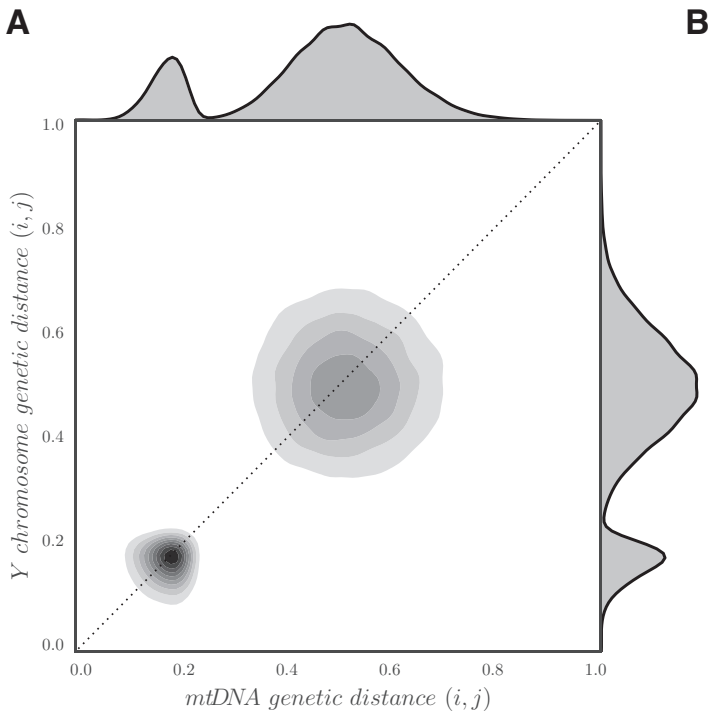

C

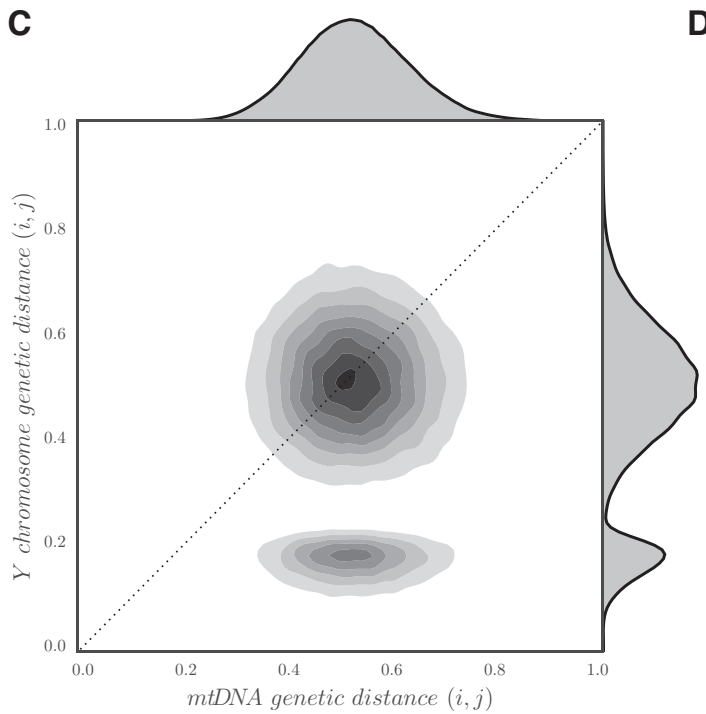

B

D
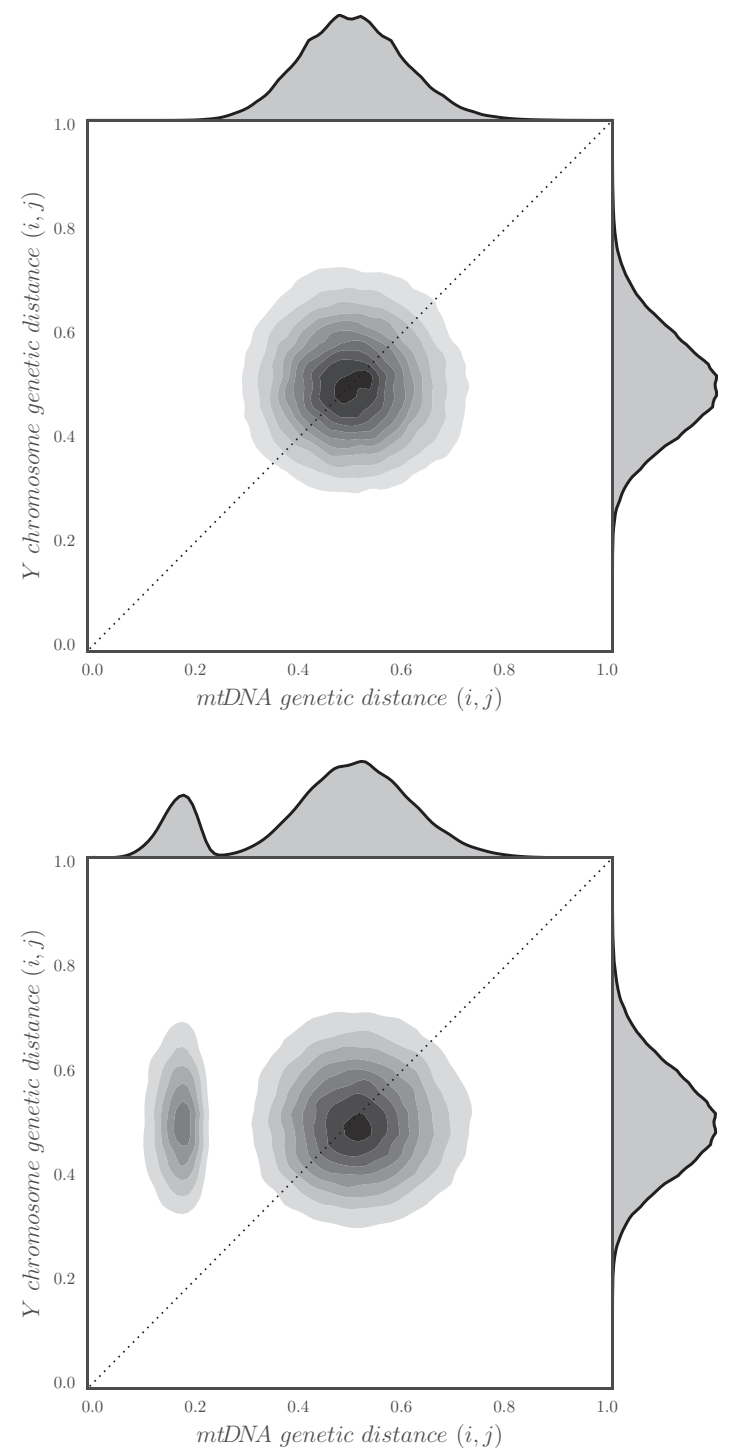

Figure 3.8: Genetic distance depending on kinship practice. The two-stage genetic distance isolation with migration model was run for a population with villages that are (A) endogamous, (B) neolocal, (C) patrilocal and (D) matrilocal. 
If both males and females marry and reside within their natal villages (Fig. 3.8A), we see two clusters of pairwise genetic distances: (1) closely related kins residing in the same village with very little genetic distance in both mtDNA and Y chromosome and (2) between village comparison of mtDNA and Y chromosome resulting to similarly higher distances. When there is no gender bias in dispersal such as in the case of neolocality, the distributions of genetic distances in mtDNA and Y chromosome are similar Fig. 3.8B. Over time, pairs of individuals become more distantly related and the genetic distances distributions broaden. In matrilocal villages, with women staying in their natal villages and men frequently dispersing, pairs of individuals remain closely related in their mtDNA but have broadly varying degree of relatedness in their Y chromosome (Fig. 3.8D). The opposite holds for patrilocal villages where men stay in their natal villages and women disperse. The absence of small genetic distances for mtDNA in Fig. 3.8C is a result of the dispersal of females.

\subsection{Kinship and language transmission}

Thus the genetic evidence suggests that sex-biased migration rates on both islands are consistent with the observed kinship rules and have persisted for many generations. Do these kinship practices sustain the association between language and genes, and in so doing, create persistent speech communities? To find out, we analyzed pairwise distance plots weighted by $d_{l}(i, j)=\cos \left(\mathbf{l}_{\mathbf{i}}, \mathbf{l}_{\mathbf{j}}\right)$, describing the degree of language sharing between individuals. A pair of individuals $(i, j)$ speaking exactly the same set of languages $\mathbf{l}_{\mathbf{i}}$ and $\mathbf{l}_{\mathbf{j}}$ will have $d_{l}(i, j)=1$. If they speak some but not all languages in common, then $0<d_{l}(i, j)<1$. If they dont speak any language in common, $d_{l}(i, j)=0$. If languages are transmitted along uniparental clades, the genetic distances between pairs of individuals who speak a common language should enhance and further reveal clusters that reflect the expected sex-biased migration patterns. To test this inference, we compare the pairwise distance plots weighted by degree of language sharing (Fig. 3.9C-D) with the unweighted plots for all pairs of individuals (Fig. 3.9A-B).

In Sumba, the signal of patrilocality is strongly enhanced (additional 25\% of sampled pairs) when only individuals who share a language are considered (compare Fig. $3.9 \mathrm{C}$ with $3.9 \mathrm{~A}$ ). All of these villages are monolingual, and all but one language 
is found in only one village, the exception being Kambera spoken in the villages of Bilur Prangadu and Mbatakapidu. Consequently, most paired individuals who speak the same language are from the same village. While this may seem to be a limitation in the data, it is actually ideal for the purpose of distinguishing whether each man inherits his language from his patriline, matriline or both. Comparing Fig. 3.9A (all pairs of Sumba men) with Fig. 3.9C (only men who share the same language), there is a very strong trend for the male children of women marrying into a community to learn the language of that community. In other words, on Sumba, language is transmitted along male lines.

Timor differs from Sumba in two key ways: our sample includes a mix of 9 matrilocal and 2 patrilocal villages, and multilinguality is common. Consequently, resulting pairwise distance show a more complex pattern of three clusters (Fig. 3.9D):

1. A matrilocal cluster $(\mathrm{m})$, comprising pairs of men who are closely related on the matriline;

2. A very weak patrilocal cluster (p), comprising pairs of men closely related on the patriline due to the two patrilocal villages in the sample; and

3. A large ambi- or neolocal cluster (an), comprising pairs of men who are less closely related on both matriline and patriline. Because the Timor sample includes both matrilocal and patrilocal communities, the unbiased ambi- or neolocal cluster is the most pronounced across the entire sample of villages on Timor.

Importantly, conditioning on the number of languages shared enlarges the matrilocal cluster by an additional $0.8 \%$ (compare Fig. 3.9D with Fig. 3.9B), the converse pattern to Sumba.

We compare in Fig. 3.10 the genetic distances in both matriline and patriline with linguistic distance, $1-d_{l}(i, j)$, defined as

$$
1-d_{l}(i, j)=1-\cos \left(\mathbf{l}_{\mathbf{i}}, \mathbf{l}_{\mathbf{j}}\right)
$$

where $\mathbf{l}_{\mathbf{i}}$ is the language vector of individual $i$ and $\mathbf{l}_{\mathbf{j}}$ is the language vector of individual $j$. A pair of individuals speaking exactly the same set of languages will have $1-d_{l}(i, j)=0$. If they both speak at least one language in common but one or both 

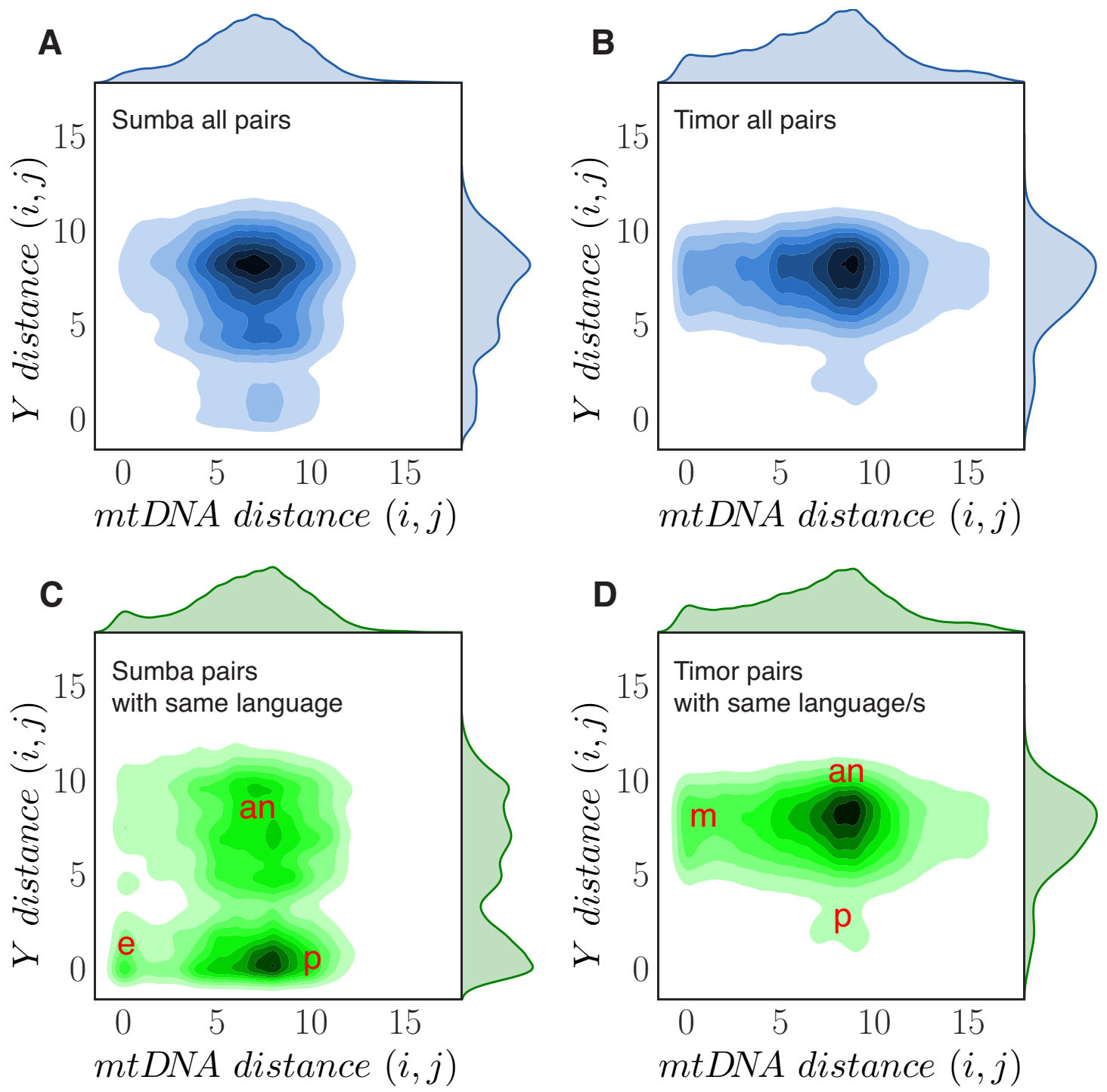

Figure 3.9: Genetic distances on Sumba (left, A\&C) and Timor (right, B\&D), both between all pairs of individuals (A-B) and only between individuals who speak a language in common (C-D). Conditioning on language sharing reveals three distinct clusters in the Sumba data (C), showing evidence of village endogamy (e), ambi- or neolocality (an) and patrilocality (p). For Timor, the high degree of multilinguality means that most pairs of individuals in B are also included in D. B and D show matrilocality (m) and ambi- or neolocality (an), and a small patrilocal cluster (p). 
of them speak other languages then $0<1-d_{l}(i, j)<1$. If they don't speak any language in common, $1-d_{l}(i, j)=1$.

In Sumba (Fig. 3.10A-B), we see the signal of monolinguality as pairs of individuals either share all their languages or do not share a language at all. The few pairs of individuals that speak the same language form a cluster with relative mtDNA genetic distance (Fig. 3.10A) of $\sim$ 8. In their Y chromosome (Fig. 3.10B), pair of individuals speaking the same languages are either closely ( $\sim 0.0)$ related or distantly related to some degree $(\sim 5-10)$.

Multilinguality is evident in Timor (Fig. 3.10C-D) where there are pairs of individuals with only some but not all languages shared. Most individuals speak the same language/s (everyone speaks Upper Tetun) and they are closely related in their mtDNA (Fig. 3.10C) but have distant relatedness in Y chromosome (Fig. 3.10D).

We find that the extent of language sharing enhances the expected sex-biased migration patterns observed on genetic distances. To further test whether the channels of kinship have guided the current of language evolution itself, we calculate the overall statistical co-phylogenetic association between languages and either matrilineal or patrilineal genetic clades.

\subsection{Co-phylogenetic association of languages and genes}

The co-phylogeny method has often been applied in functional ecology to find links between species traits and environmental variables and thus explain observed biological processes in ecosystems [57]. More recently, similar methods have been applied in the context of host-parasite co-evolution [58] and cultural traits [59]. The evolution of language is analogous to parasites carried by their human speakers, whose genes evolve in a parallel process. Language traits evolve as the environmental and genetic conditions of the speech communities evolve. Using the co-phylogeny framework, we test the significance of a global hypothesis of co-evolution between genes and language [58].

Co-phylogenetic analysis requires specifying a binary association link matrix indicating which of the locally spoken languages each sampled individual speaks (e.g., for Timor: Bunak, Betun, Dawanta, Kemak and/or Upper Tetun; for Sumba: lan- 

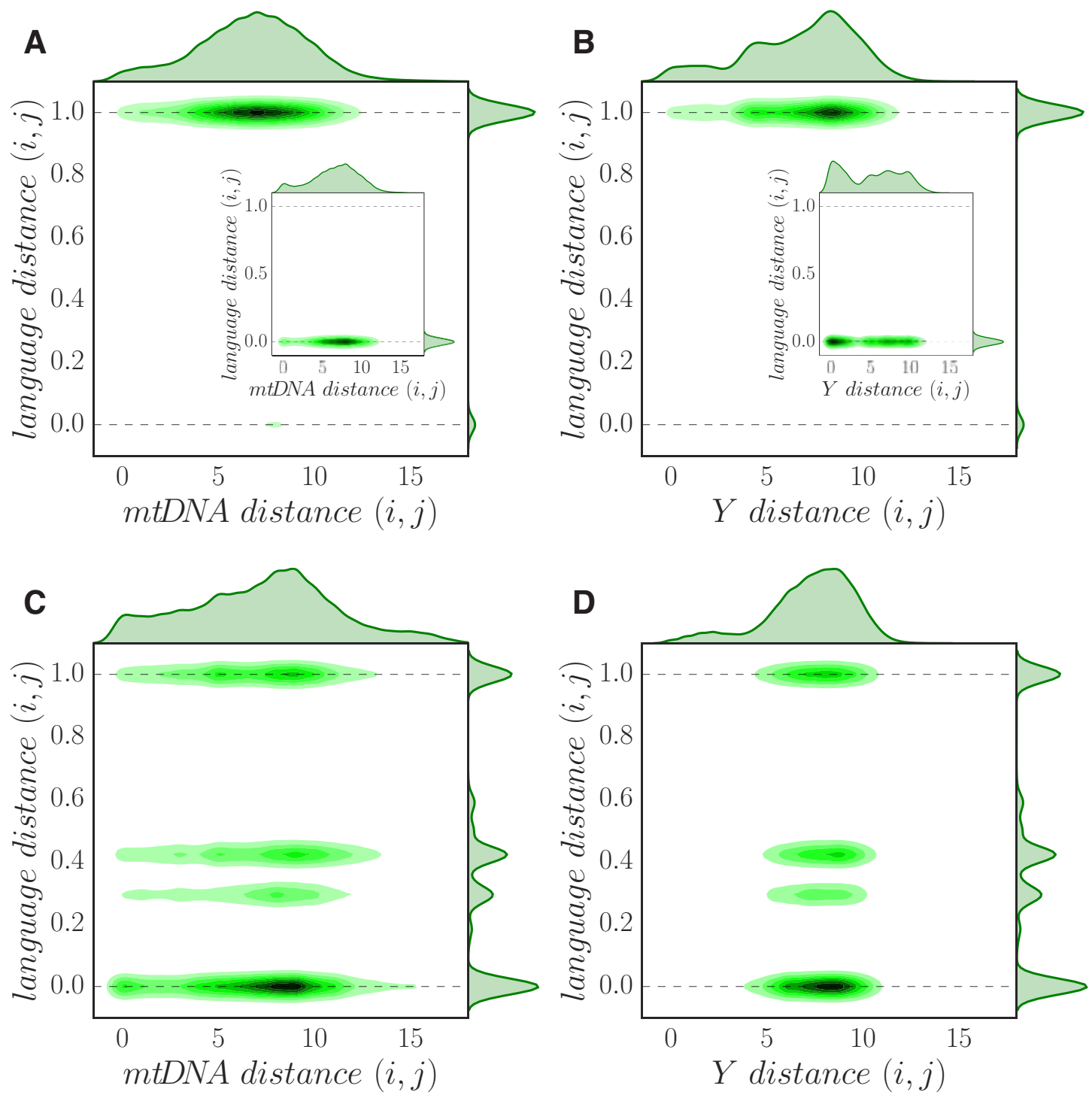

Figure 3.10: Linguistic and genetic distances. The kernel density of the linguistic and genetic distances of all pairs of individuals is shown. (A) In Sumba where villages are monolingual, only a few pairs with mtDNA distance $\sim 8$ speak the same language. (B) Looking at the Y chromosome in Sumba, pairs of individuals speaking the same language form two clusters of close $(\sim 0.0)$ and slightly distant $(\sim 5-10)$ relatedness.(C) In Timor, there is a high probability of finding pairs of individuals that are both closely related in their mtDNA and in the languages they speak. (D) Most pairs of individuals in Timor have a distant degree of relatedness in their $\mathrm{Y}$ chromosome but speak similar set of languages. 
guage spoken in their monolingual village). In Timor, language fluency was assessed for each participant on a three-point ordinal scale ('Understand', 'Understand some', 'Do not understand') during a survey administered to each participant. The first two categories were then coded as positive indication of ability with the corresponding language for the purposes of the cophylogenetic analysis.

\subsubsection{Statistical test for gene-language coevolution}

To test whether a significant association exists between the evolution of genes and languages, we employ statistics that are functions of the gene and language phylogenetic trees and their association links (i.e., the list of which individuals speak which languages) [58]. In this statistical test, we examine the significance of congruence, if any, between the gene and language trees. If genes and languages occupy corresponding positions in both phylogenetic trees with a significant degree of congruence, we reject the global null hypothesis that their evolution has been independent.

\subsubsection{ParaFit statistic}

Evaluating the global hypothesis requires describing the process with three pieces of information: i) the gene phylogeny $\mathbf{G}$, ii) the language phylogeny $\mathbf{L}$, and iii) the association between genes and languages LG.

The cophylogeny matrices $\mathbf{L}$ and $\mathbf{G}$ (Fig. 3.11) represent the principal coordinates of the linguistic and genetic distances of individuals along their respective trees. The association matrix LG is a binary link specifying the languages spoken by each individual, and $\mathbf{L G}^{\prime}$ is the transpose of $\mathbf{L G}$. To calculate the congruence of the gene and language trees, we define the fourth-corner statistics matrix $\mathbf{D}$ [58] as

$$
D=G L G^{\prime} L
$$

From D, we define the global association ParaFitGlobal statistic, to test the genelanguage coevolution hypothesis, as the sum of squares of $d_{i j}$

$$
\text { ParaFitGlobal }=\operatorname{trace}\left(\boldsymbol{D}^{\prime} \boldsymbol{D}\right)=\sum\left(d_{i j}{ }^{2}\right)
$$




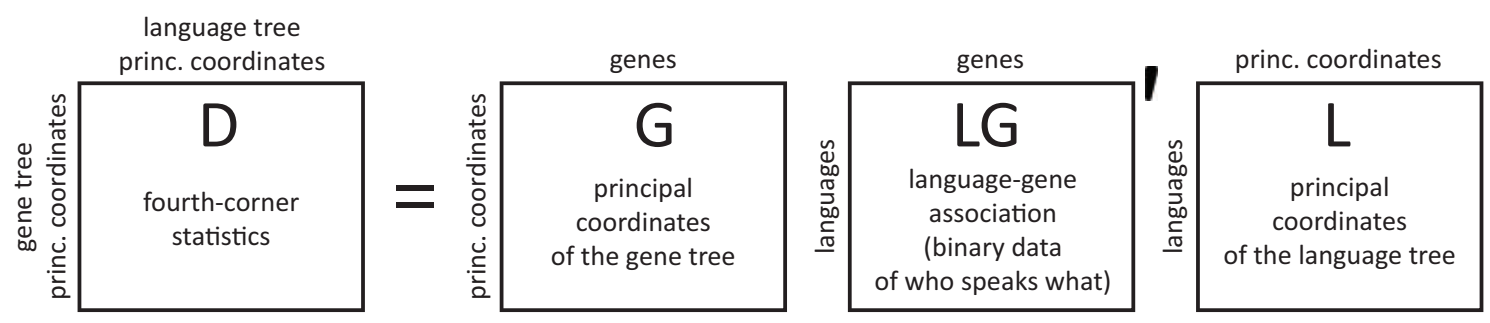

Figure 3.11: Fourth-corner matrix. Statistics describing gene-language associations.

\subsubsection{Permutation Model}

To test whether the association between genes and languages is significant, we calculate ParaFitGlobal on the original data, as well as on the permuted data. Permutation is performed by shuffling the columns of $\mathbf{L G}$ as shown in Fig. 3.12. Randomizing the columns is akin to shuffling the genes labels in the link matrix, which removes the association, but preserves the extent and degree of multilinguality. Permutation was used as the null model because if kinship did not systematically channel languages and genes then the languages spoken by every individual would just be randomly assigned.

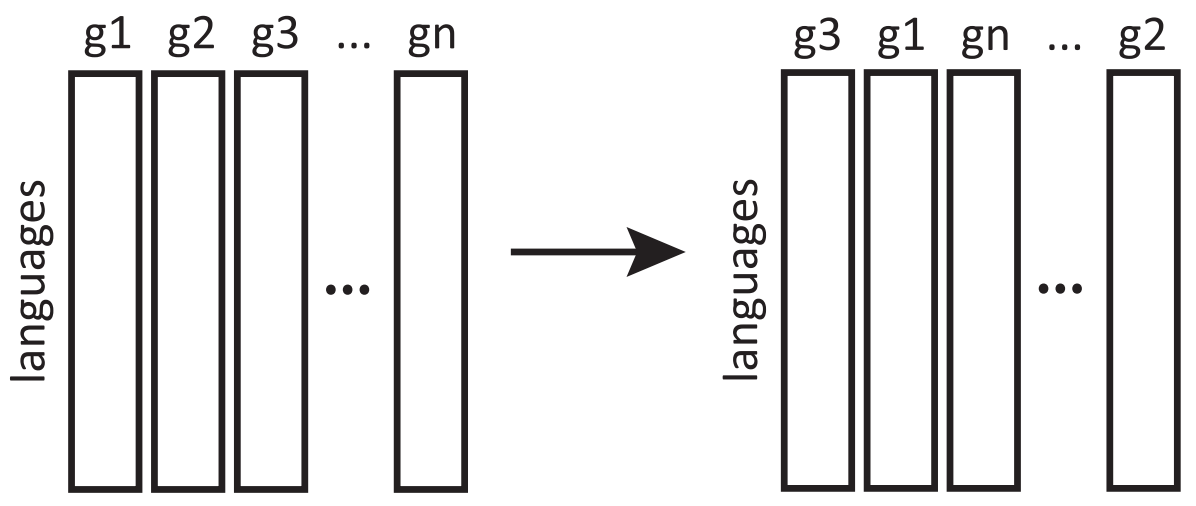

Figure 3.12: Permutation model. Columns of the $L G$ matrix are shuffled to remove associations, while preserving the number of multilingual individuals and the number of languages they speak.

The observed association between genes and languages is then determined using $Z$ score, which takes the distribution of ParaFitGlobal values of different permuted realizations of the data, ParaFitGlobal ${ }^{\text {perm }}$, and calculates the number of standard deviations, s, ParaFitGlobal ${ }^{\text {data }}$ (i.e. ParaFitGlobal value of the original data) 
is away from the mean of $\left\{\right.$ ParaFitGlobal $\left.{ }^{\text {perm }}\right\}$. The $Z$ score is taken over $2^{5}$ randomizations $r$, each with 999 permutations $p$ of ParaFitGlobal Perm $^{\text {. }}$

$$
Z=\frac{\text { ParaFitGlobal }^{\text {data }}-\frac{\sum_{r, p} \text { ParaFitGlobal }_{r, p}^{\text {perm }}}{r+p}}{s\left(\left\{\text { ParaFitGlobal } l_{r, p}^{\text {perm }}\right\}\right)}
$$

\subsubsection{Results of co-phylogenetic association of languages and genes}

We calculate the overall statistical co-phylogenetic association between languages and either matrilineal or patrilineal genetic clades in Table 3.1. In patrilocal Sumba, a far stronger association is seen between genes and languages for the $\mathrm{Y}$ chromosome $(Z=67.7$, shown by grey shading in Table 3.1$)$.

Table 3.1: $Z$ scores of gene-language associations. Comparing within each group of villages (columns), stronger gene-language associations (shading) are found for $\mathrm{Y}$ in all Sumba villages, mtDNA in all Timor villages, mtDNA in matrilocal Timor villages, and $\mathrm{Y}$ in patrilocal Timor villages.

\begin{tabular}{c|ccc|ccc} 
& \multicolumn{3}{c}{ Sumba } & \multicolumn{3}{c}{ Timor } \\
\cline { 2 - 7 } & all & matrilocal & patrilocal & all & matrilocal & patrilocal \\
\hline mtDNA & 8.32 & - & 8.32 & 5.43 & 6.62 & 1.01 \\
Y & 67.7 & - & 67.7 & 3.85 & 4.04 & 2.67 \\
\hline
\end{tabular}

A similar pattern (stronger $Z=2.67$ for $Y$ ) exists for the two patrilocal villages in the Timor sample, while the opposite pattern (stronger $Z=6.62$ for mtDNA) is found for the matrilocal villages of Timor. Significant associations exist in cases where we are tracking descent through transmission channels dictated by their kinship practice (i.e. matriline ancestral descent in matrilocal villages and patriline ancestral descent in patrilocal villages). The absence of language-gene association for mtDNA in patrilocal villages indicates high dispersal of females originating from the patrilocal villages of Timor. However, some association is found between language and Y chromosome suggesting that either (1) men from matrilocal villages rarely disperse, or (2) most of the men that immigrate to the matrilocal villages come from only few patrilocal villages making it more probable that they come 
from the same genetic clade.

\subsection{Summary and Conclusions}

Associations between genes and languages have been found even in the presence of sustained migration among communities. We compare phylogenies of gene and language trees to explore the origins of this association. In traditional tribal societies, marriage customs channel language transmission. When women remain in their natal community and men disperse (matrilocality), children learn the language of their mothers, and language correlates with the maternally inherited mitochondrial DNA. When men stay in their natal community and women disperse (patrilocality), the opposite pattern holds. Kinship rules that dictate postmarital residence can persist for many generations, and determine population genetic structure at the community scale. The association of languages with clades created by kinship systems provides information not only about language transmission, but also about the structure and persistence of social groups.

Whereas oral languages seldom persist for more than 2 kya, speech communities last much longer. The implication is persistence of matrilocal speech communities. Patrilocality merely disrupts this pattern. In each generation there is an opportunity to weaken or scramble the correlation between languages and uniparental lineages through a migration event, which occurs when children learn a different language than one of their parents. Maximum likelihood estimates were calculated for the frequency of migration. The lack of correlation between patrilines and languages indicates that widespread host switching occurred at least once in the past, but does not tell us when or how often.

These results do not imply that post-marital residence was always matrilocal. Instead they indicate an enduring relationship between women and their speech communities. That is the observed pattern today: these Timorese villages typically include a mix of mitochondrial haplogroups. Not infrequently, women marry out of their natal villages, but remain within the circle of communities that form their speech community. The results of the co-phylogenetic analyses pertain to the persistence of speech communities with shared cultures, not co-residential social groups.

The persistence of matrilocal speech communities also has implications for cul- 
tural evolution. In modern times, languages are often shared across many societies. But in exclusively oral premodern societies, the entire repertoire of symbols that constitute a shared culture are encoded and transmitted in its language. Consequently, tracing the history of the language of an oral pre-modern society is equivalent to tracing the history of the culture it encodes in the brain. The prolonged continuity of matrilocal speech communities would provide a social context enabling languages and cultures to evolve in situ. Occasional host switching of languages between uniparental descent groups provides evidence for social and cultural contact between these communities.

Languages are transmitted through channels created by kinship systems. Given sufficient time, these kinship channels can change the genetic and linguistic structure of populations. In traditional societies of eastern Indonesia, finely resolved co-phylogenies of languages and genes reveal persistent movements between stable speech communities facilitated by kinship rules. When multiple languages are present in a region and postmarital residence rules encourage sustained directional movement between speech communities, then languages should be channeled along uniparental lines. We find strong evidence for this pattern in 982 individuals from 25 villages on two adjacent islands, where different kinship rules have been followed. Core groups of close relatives stayed together for generations, while remaining in contact with, and marrying into, surrounding groups. Over time, kinship systems shaped their gene and language phylogenies: consistently following a postmarital residence rule turned social communities into speech communities.

\subsection{Future Work}

Our framework of calculating for the probability of shared heritage can be applied to genetic and linguistic data taken from other areas. This can provide a first step in visualizing the extent of shared heritage. Furthermore, the method of looking at the joint plots of genetic distances to reveal language transmission through kinship channels has previously not been done in other studies involving genetic data. Applying this method to published genetic data can further give insight as to the marriage and kinship customs practiced in these areas.

The proposed alternative kinship IM model which is a two-stage extension of 
the existing coalescent IM model has not been tested with the data. With enough resources, the validity of this more realistic kinship IM model should be tested. Does it show better fit with the data? What is the extent of overfitting given that this alternative model has more parameters? Are the results we get from the tested kinship IM model comparable with that we can get with the alternative kinship IM model?

Finally, all the methods presented here specifically the co-phylogenetic association method can be applied to other co-phylogeny studies. Co-phylogeny exists not only between genes and languages but also between genes and a number of other cultural and biological systems. As an example, one can try to examine generational disease transmission by looking at the co-phylogeny of genes with markers of disease immunity. 


\section{Chapter 4}

\section{Coupling of Language and Gene Trees}

Geneticists routinely test for the association of languages and genes. The association between genes and languages is often transient [60], but at small spatiotemporal scales can be strong. Since Cavalli-Sforza first identified this pattern at the global scale, correlations between languages and genes have often been found at regional and local scales. By jointly analyzing phoneme inventories and microsatellite polymorphisms in 246 populations worldwide, Creanza et al. found that there's a correspondence in the regional geographic axes of greatest genetic and linguistic variations suggesting a correlated spread of languages and genes among communities with shared history [61]. What evolutionary processes cause this regional association?

In the simplest case, communities retain their languages, resulting in a perfect correlation between language and gene trees that persists even as they accumulate variation. However, if dispersal is frequent, the correlation between languages and genes breaks down. Empirically, we know that dispersal is common, yet correlations are frequently observed between languages and genes at the population level. To investigate this conundrum, we examined the consequences of dispersal in traditional societies. We consider the four possibilities of dispersal based on kinship practice (1) endogamy, (2) neolocality, (3) patrilocality or (4) matrilocality. In traditional and pre-modern societies where language diversity is high, these dispersal practices have different consequences.

In matrilocality wherein women remain in their natal villages and men disperse, 
children will learn the language of their mothers. In such a case, if men often marry outside the radius of their mother's speech community, language correlates with the maternally inherited mitochondrial DNA (mtDNA) but not with the paternally inherited Y chromosome as shown in the previous chapter. Evidence indicating intermarriages between speech communities is found in the PHOIBLE language database as its worldwide median of neighboring languages within a radius of 75 $\mathrm{km}$ is three $(>1)$ [61]. If men stay in their natal village and women disperse (patrilocality), the opposite pattern should hold. Language should correlate with the paternally inherited Y chromosome but not with the maternally inherited mtDNA.

We tested this model on the eastern Indonesian island of Timor (Fig. 1.3), in a district where five languages are spoken in ten traditional villages. Two villages are patrilocal and the rest are matrilocal. When the Austronesians arrive in the islands, they brought their language with them but nearly all of the islands were already inhabited by people who spoke their own languages. Yet nearly all of those original languages disappeared. Within a few thousand years, Austronesian languages carried by small numbers of colonists replaced the indigenous languages on hundreds of islands across half the globe. What were the social mechanisms responsible for this?

\subsection{Co-evolution of languages and genes}

We find in the previous chapter significant associations between languages and genes for mtDNA in matrilocal villages and for the $\mathrm{Y}$ chromosome in patrilocal villages. When did these associations appear and how did they disappear? By exploring the correspondence between branches in the language and gene trees, we can track the nature of the association through time. Fig. 4.1 shows how correlations between languages and populations can appear, persist or be lost.

Using a cophylogenetic approach, it is possible to approximately identify these occurrences. Over time, diversity increases in both languages and genetic populations, making it possible to date branching events. When branching occurs, language and gene trees can split at the same time and continue to diverge in a process analogous to cospeciation resulting in strong correlation (Fig. 4.1A). Languages can also be adopted from other communities, where they may replace an existing language 
A

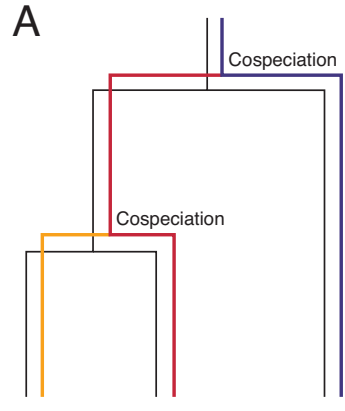

B

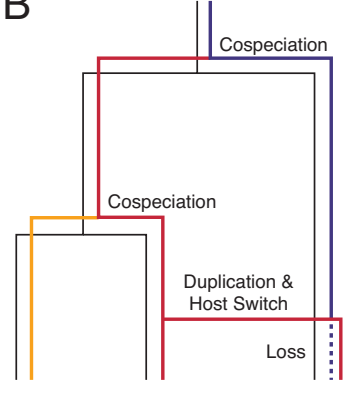

C

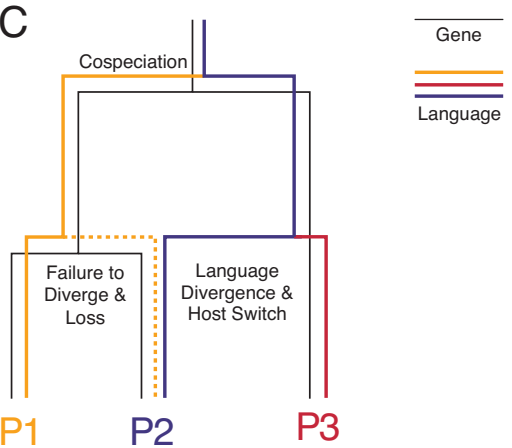

Figure 4.1: Languages and genes cophylogeny correlation. (A) Strong correlation of languages and genes exists as a result of only cospeciation events. (B) Only some correlation remains if in between cospeciation events, duplication, host switching or loss happens. (C) Almost all correlation is lost if frequent host switching and loss occurs.

or add to the current language pool. When this happens sparsely, some correlation remains (Fig. 4.1B), but frequent language switches and losses completely break down the correlation (Fig. 4.1C).

The strong associations found for mtDNA in matrilocal villages and Y chromosome in patrilocal villages correspond to the case of frequent cospeciation illustrated in Fig. 4.1A. In patrilocal villages, frequent dispersal of women result to widespread language switching and loss leaving no signature of language and gene associaiton as in Fig. 4.1C. In matrilocal villages, however, although men are allowed to disperse, some correlation for the $\mathrm{Y}$ chromosome remain similar to Fig. 4.1B suggesting either (1) dispersal of men from matrilocal villages is not frequent or (2) groups of men from patrilocal villages who chose not to adhere to their villages' kinship practice migrated to matrilocal villages. The latter may occur considering that there are only two patrilocal villages as opposed to eight matrilocal villages. When considering all villages, language globally correlates more with matriline genetic descent than the patriline genetic descent. This reflects the more widespread practice of matrilocality over patrilocality. 


\subsection{Cophylogeny reconstruction of language and gene trees}

In the island of Timor, individuals practicing matrilocality outnumbers those practicing patrilocality leading to movements that favor the persistence of speech communities in women than in men. Larger speech communities (i.e. genetically closely related individuals speaking a common language) are found in the matriline descent than in the patriline descent (Fig. 4.2). The larger speech communities in the matriline descent has language and gene correlation that persists longer hence leaving a stronger association signal.

\subsubsection{Jane method of co-phylogeny reconstruction}

Jane is a software tool developed by Libeskind-Hadas to reconstruct historical associations of phylogenetic trees believed to have coevolved. We use this software to reconcile language and gene trees and map which nodes in this pair of trees were subject to simultaneous biologically plausible events. Each plausible event is assigned a cost which determines how the languages evolved following the evolution of the genetic tree.

The cophylogeny reconstruction problem is described by the six-tuple $\left(H\left(V_{H}, E_{H}\right)\right.$, $\left.P=\left(V_{P}, E_{P}\right), t_{H}, t_{P}, \phi, \kappa\right)$ where $\phi: V(P) \rightarrow V(H)$ is the network mapping that minimizes $\kappa$ costs of events. The plausible events that can occur as languages coevolve with genes are

The cophylogenetic network mapping $\phi$ is recovered through dynamic programming using genetic algorithm. The cost of each event is any of $\{0,1,2\}$. The program is ran over all possible combinations of event costs. The reconstruction selected is that which gives the smallest total sum of event costs.

\subsubsection{Co-phylogeny of languages and genes}

Fig. 4.4 shows the language and gene co-phylogenies for both mtDNA and Y chromosome genetic trees, from which several patterns emerge. First, host switching occurs primarily deep in the past near the roots of the trees. Second, losses occur at similar rates throughout the length of both trees. We see that the global patterns 

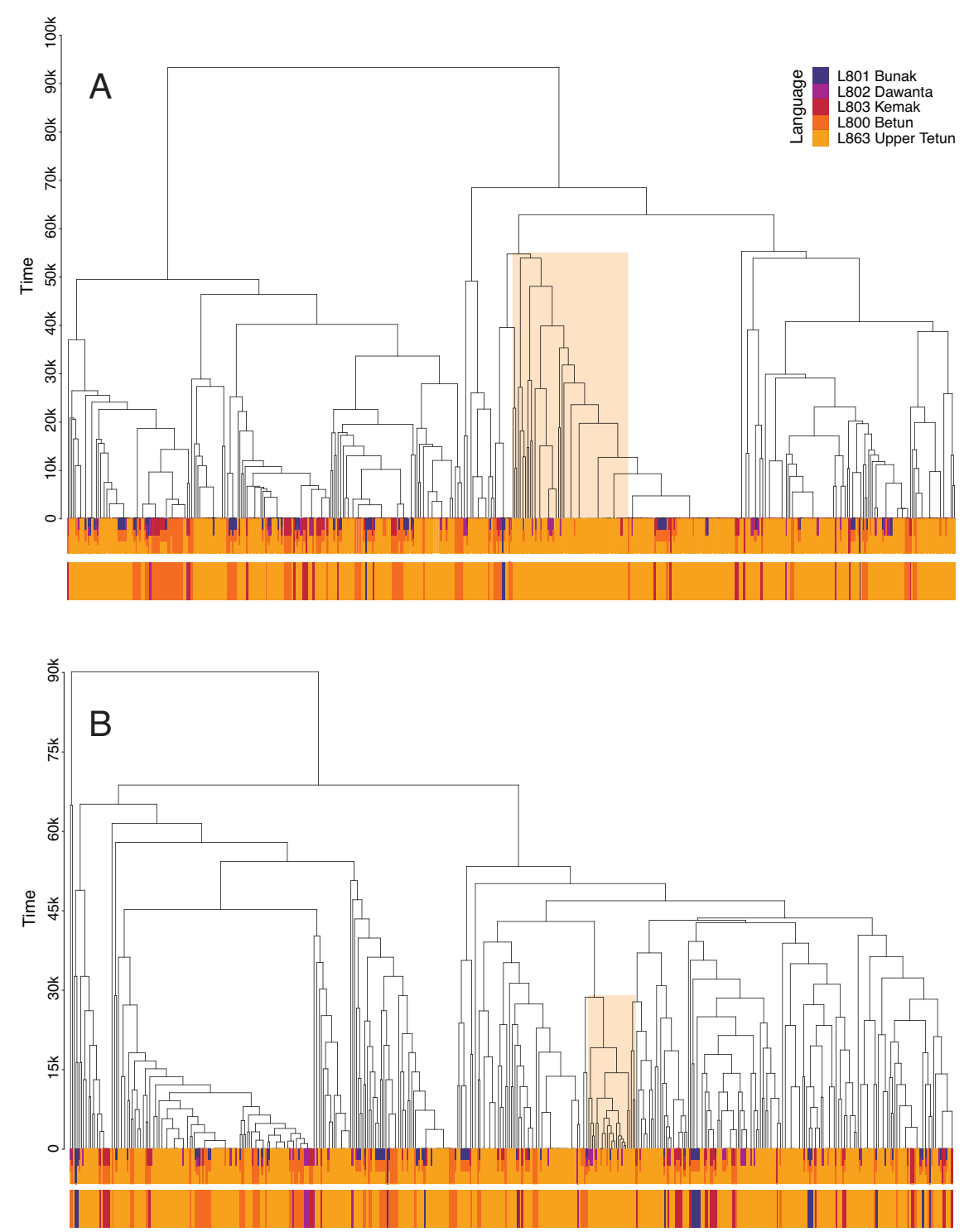

Figure 4.2: Languages in the (A) matriline (mtDNA) and (B) patriline ( $Y$ chromosome) phylogenies. The first color bands in (A) and (B) are all the languages spoken by every individual in the phylogenies. Multilinguality is represented by multiple colors in the first color bands below the individuals. The second color bands visually represent the size of speech communities. The largest speech community in (A) is bigger (69 individuals) with an older most recent common ancestor ( 55 kya) as compared to the largest speech community in (B) with 26 individuals genetically belonging to two sub-clusters with most recent common ancestors at $\sim 29$ kya and $\sim 20$ kya. 


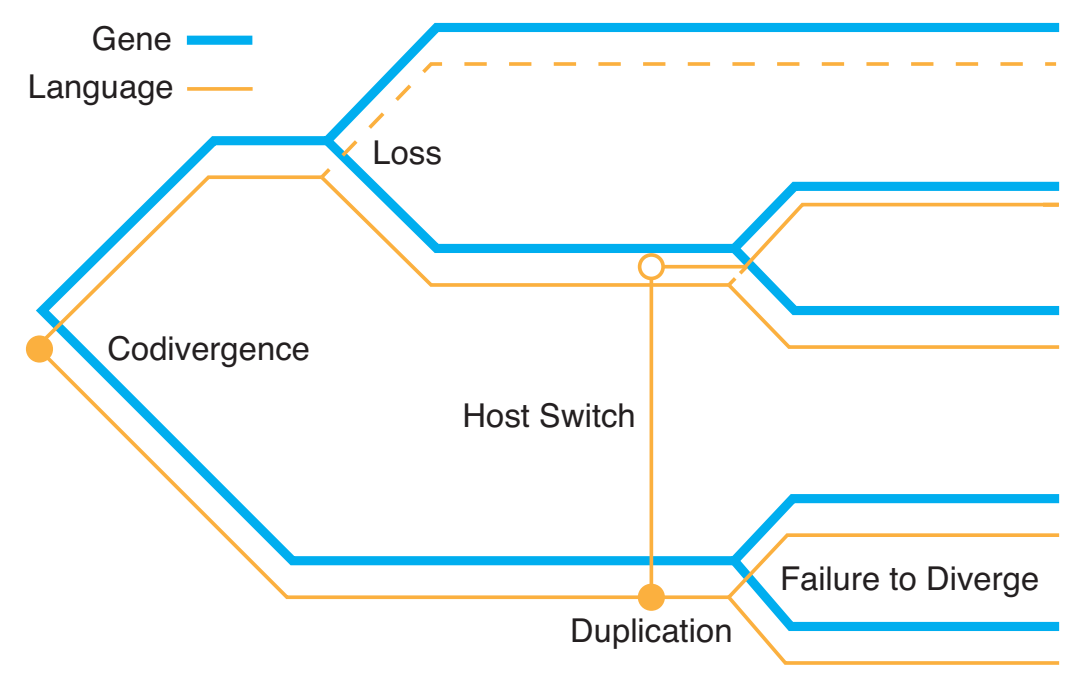

Figure 4.3: Cophylogenetic events. There are five plausible events that languages can experience as it evolve with genes: (1) codivergence, (2) loss, (3) duplication, (4) host switch and (5) failure to diverge).

of language switches and losses can be traced to differences in the finely resolved language movements in patrilocal versus matrilocal villages.

\subsection{Host switching and persistence of speech com- munities}

Host switch and loss events pertain to situations wherein a group of speakers either acquire an additional language or adopts a new one and cease using their current language. From the co-phylogeny reconstruction of Jane, we can calculate for the rates of host switching and loss for both parental lines (mtDNa for matriline and $\mathrm{Y}$ chromosome for patriline) under both matrilocality and patrilocality kinship practices

In Table 4.1, we see that for the matrilineally transferred mtDNA, there is less host switching and loss for matrilocal villages which is the same group of individuals that showed strong association of genes with languages in Chapter 3. Similarly, for the patrilineally transferred Y chromosome, patrilocal villages with strong association of languages and genes show a lesser rate of host switching and loss.

Interestingly, speech communities where individuals share a common language while also having a common ancestor in their mother side are more likely to be 

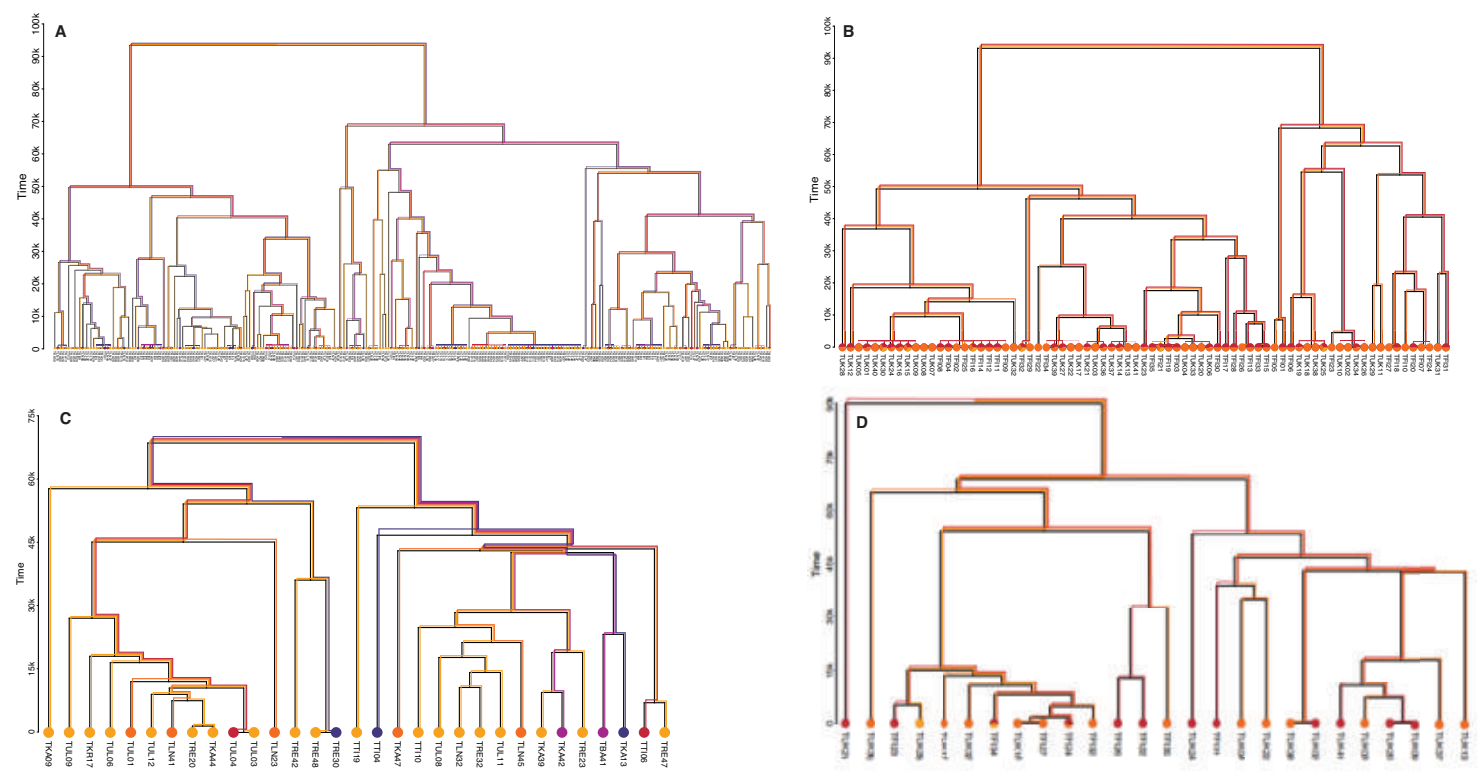

Figure 4.4: Language and gene trees co-phylogeny. The co-phylogeny reconstruction from Jane of significant associations is shown for (A) mtDNA matrilocal villages, (B) mtDNA patrilocal villages, (C) Y chromosome matrilocal villages and (D) Y chromosome patrilocal villages.

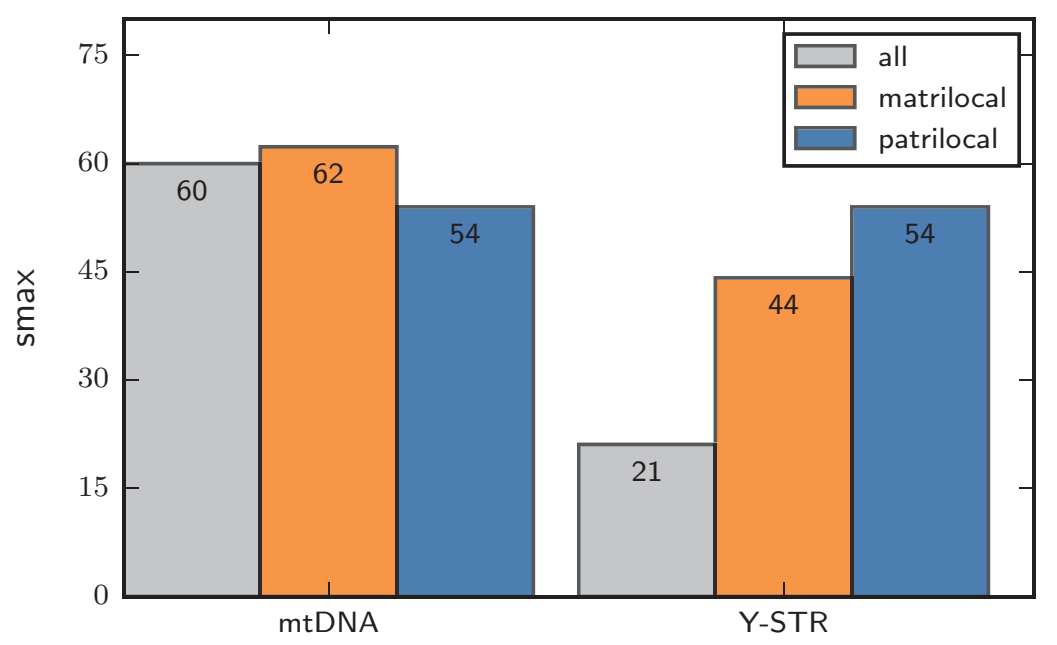

Figure 4.5: Maximum size of speech community. Matrilocal villages are more likely to have a bigger speech community in their mtDNA while patrilocal villages are more likely to have it bigger in their Y chromosome. 
Table 4.1: Host switching and loss rates per generation. Rates are computed for the relatively recent past of 10 kya to present. Cells in gray are the cases that also shows strong association of languages and genes.

\begin{tabular}{ccc} 
& matrilocal & patrilocal \\
\hline mtDNA & 0.46 & 4.21 \\
Y chromosome & 1.13 & 1.04 \\
\hline
\end{tabular}

bigger for matrilocal villages. Conversely, speech communities having a common ancestor in their father side is bigger for patrilocal villages.

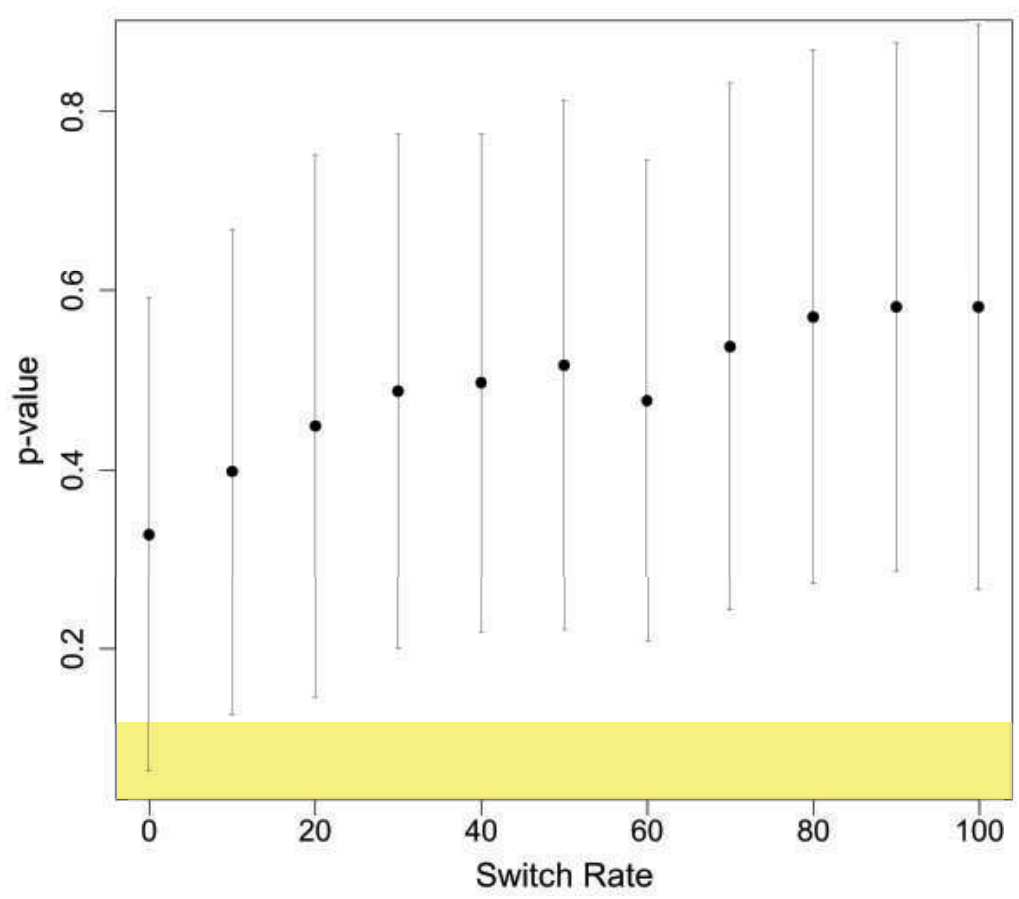

Figure 4.6: p-value for the different host switching rates. Host switching must occur below $5 \%$ for the p-value of language-gene association to be significant.

We also calculated for the degree of association of languages and genes under different rates of host switching. We show in Fig.4.6 that languages and genes can only be associated ( $\mathrm{p}$-value $<0.1$ ) if the host switch rates are below $5 \%$. We see in Table 4.1 that none of the switch rates exceed $5 \%$ and as such we observe association of langauges and genes albeit at varying degree of significance.

For the case of mtDNA patrilocal villages, the switch rate is almost $5 \%$ which 
might be why we see no association of languages and genes for this case. The degree of association of genes and languages is influenced by the extent of the persistence of languages as suggested by the expected speech community size and rate of host switching and loss.

\subsection{Language switching between genetic clades}

We have seen that kinship creates channels for language transmission along uniparental clades, and that this occurs over time scales that structure language sharing between related individuals. Yet it remains unclear how long these associations persist. The phylogenetic trees for mtDNA and Y chromosome (Fig. 4.2) have roots in the very distant past, long before any conceivable relationship to the languages spoken today could have existed. Most of these genetic lineages are from the region's first settlers, before Austronesian languages were introduced 5, 000 years ago. Intriguingly, however, some information about the association between languages and genetic clades in the deep past is preserved by the branching points where languages either become attached to, or leave genetic clades. Using this information, we can estimate "host switching" probabilities for the observed gene-language tree associations. We adapt this idea from co-phylogenies in ecology where host switching refers to movements of parasites between host species. Here, hosts are people and parasites refer to languages [57, 60].

To estimate "host switching" probabilities, we assume that both gene and language trees are accurately reconstructed, and seek to generate a one-to-one mapping of the gene and language trees' branching points describing how the languages were transmitted, leading to the current language/s spoken at each leaf of the gene tree. A plausible model shown in Fig. 4.7 predicts the language/s spoken at every branch in the gene tree at generation $t$. This stochastic simulation is run forward in time over the trees using two rules. First, where the language tree branches into two daughter languages, all genetic clades that speak the ancestral language are randomly assigned to one of the daughter languages. Second, in each generation, there is a probability $\alpha$ that a given clade switches to a new language chosen from among the languages that exist in the population at that time. That is, a proportion of lineages $\alpha$ switches to a new language at each generation. These two rules provide 
a simple model of language diversification and host switching (Fig. 4.7) that is sufficient to reconstruct patterns of language sharing observed in the present. Variant of this gene-language co-evolution model yield qualitatively similar results.

\subsubsection{Language switching on genetic trees}

A plausible stochastic model of language transmission along the branches of the gene tree ran forward in time (starting from the gene tree root, $t_{0}$ in Fig. $4.7 \mathrm{C}$ ) involves the following process performed at each generation of 25 years: (1) Determine the genetic clades and languages at generation $t$ (e.g. 2 genetic clades and languages $L_{12}^{*}$ and $L_{3}$ at $t_{2}$ in Fig. 4.7B-C); (2) If a language branches at generation $t$, each genetic branch that carried the ancestral language at $t-1$ is randomly assigned one of the two new languages (e.g. at $t_{2}$ in Fig. $4.7 \mathrm{C}, L_{12}^{*}$ is assigned to first branch and $L_{3}$ to second branch); (3) Each genetic branch then switches to a different language within the current pool of languages with a probability $\alpha$ (e.g. genetic branch $G_{2}$ switches to language $L_{3}$ at $t_{4}$ ).

A

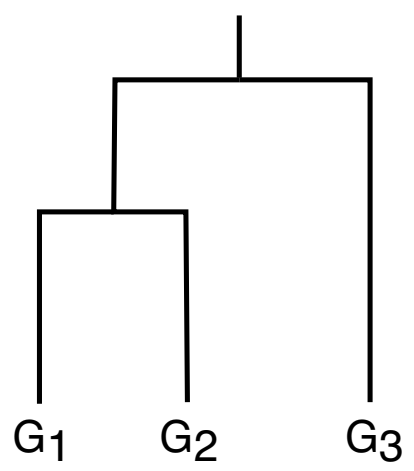

B

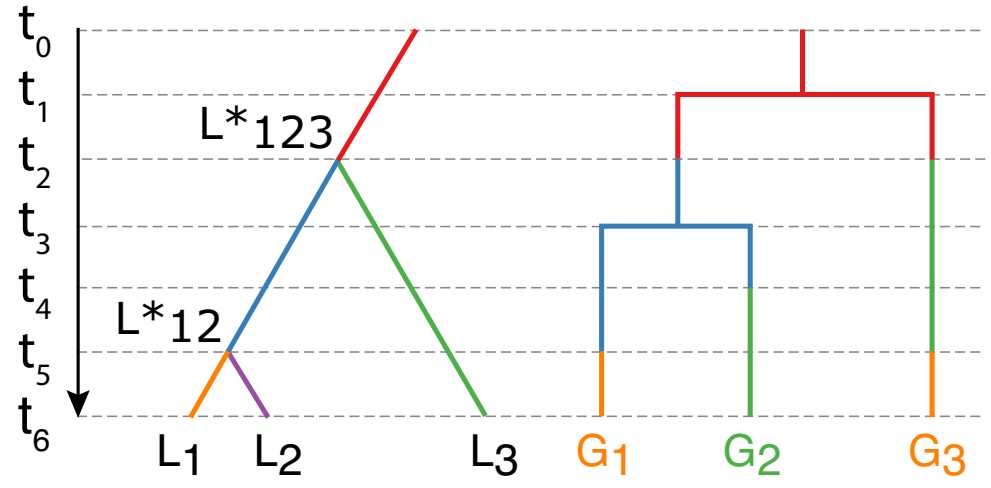

Figure 4.7: Language switching in a gene tree conditioned on a language tree. Shown in (A) is an unannotated gene tree, (B) a language tree and (C) an example of a simulated language-annotated gene tree. Language diversifies during language branching ( $L_{123}^{*}$ splits into $L_{12}^{*}$ and $L_{3}$ at $t_{2}$ ), and during host switching, as in the branches leading to $G_{2}$ (language switch at $t_{4}$ ) and $G_{3}$ (language switch at $t_{5}$ ).

From this model, a mapping of the language/s spoken at present by every individual in the gene tree (Fig. 4.7C) was generated. We then perform ParaFit analysis 
$[58,57]$, a standard co-phylogeny statistical test, to examine the extent of congruence, if any, between the gene and language trees given the mappings simulated from the model. This process was repeated for many gene-language simulations across the range $\alpha=[0,100]$ where $\alpha=0$ indicates no language switching and $\alpha=100$ means all lineages switch language every generation. The average $Z$ of each $\alpha$ is then compared with the $Z$ of the observed data to find the degree of language switching that best fits the data.

We investigated eight co-phylogenies separately as indicated in Fig. 4.8. In each case, we find that low rates of host switching $(<0.5 \%)$ are necessary to generate co-phylogenies with strengths of association like those seen in the observed data. Language switching rates are lower for the non-dispersing sex. This is expected as there are fewer opportunities for the non-dispersing sex to be exposed to new languages, so the rate of language switching is lower. These low language switching rates strengthen the association between genes and languages.

The model of language switching between genetic clades as shown in Fig. 4.7 was repeated for simulations across a range of values for language switching $\alpha$. From these, we obtained a distribution of $Z$ scores and identified the $\alpha$ values that yield $Z$ scores similar to those seen in the observed data. This simulation model is sufficient to generate plausible patterns of language sharing in Sumba, where individuals are monolingual. However, multilinguality is common in Timor, and we therefore modified the model such that individuals have a list of languages. When a new language is introduced (i.e., ancestral language branches into daughter languages), or language switching occurs (as determined by $\alpha$ ), there is now a fixed probability $\beta$ that the new language is appended to the language list rather than replacing the language list. We are primarily interested in fitting the language switch rate $\alpha$, and so only a limited range of $\beta$ values is explored. In Fig. 5 , we take $\beta=\alpha$. Other $\beta$ values yield qualitatively similar results, with all values leading to $Z$ score convergence at $\alpha<5 \%$. 


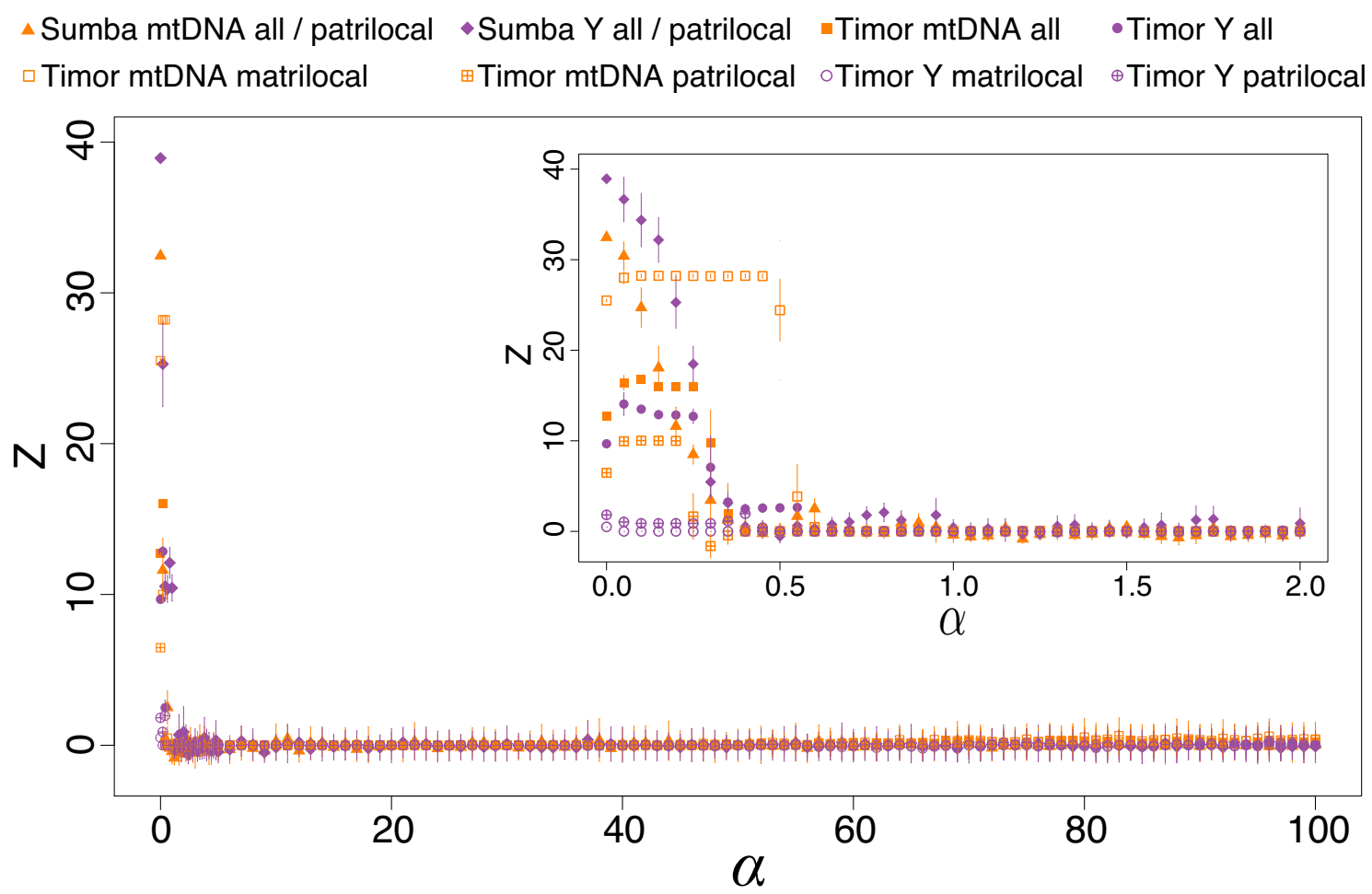

Figure 4.8: Language switching rates. The $Z$ score measure of association between gene and language phylogenies on Sumba and Timor for different language switching rates $\alpha$. All cases independently converge and abruptly lose gene-language associations, behaving similar to randomized cases, when the language switch rate exceeds $\sim 0.5 \%$. 


\subsubsection{Alternative model of language switching between ge- netic clades}

An alternative plausible stochastic model of language transmission along the branches of the gene tree ran forward in time (starting from the root of the gene tree) involves the following process performed at each generation $t$ of 25 years:

1. Setting parameters - Determine the genetic clades and languages at generation $t$

2. Cospeciation - If language branches at generation $t$, each genetic branch that carried the ancestral language at $t-1$ is randomly assigned one of the two new daughter languages. Genetic branches associated with a language that does not branch inherit the language/s spoken by that branch at time $t-1$; and

3. Language switch - Each genetic branch then switches to a different language within the current pool of languages with a probability $\alpha$.

In the case of multilinguality such as in Timor, every individual has a list of languages and the set of languages spoken by an individual is determined following these additional rules:

4. Parent language replaced by daughter language during cospeciation - Whenever language branching occurs, for every genetic branch that spoke the ancestral language at $t-1$, one of the daughter languages replaces the ancestral language in the current list of languages of the genetic branch; and

5. Additional language during switch - When a genetic branch switches to a language not in its language list at $t-1$, it retains the other languages it already speaks at $t-1$.

For this alternative model, language switching rates $\alpha$ converge to $1-5 \%$ per generation for all trees examined as shown in Fig. 4.9. This means that under this alternative model, the "half life" of a language on a lineage is 325-1, 700 years.

The difference of this alternative model with the model used in Fig. 5 is in steps 4 and 5. While in the model used in Fig. 5, the new language is appended with a probability of $\beta$, in this alternative model, (i) the chosen new daughter language 
always replaces the ancestral language in step 4; and (ii) the language chosen during switching in step 5 is always appended to the genetic branch's language list. That is, in the main model, $\beta$ is allowed to vary while in this alternative model, $\beta$ is always 1.

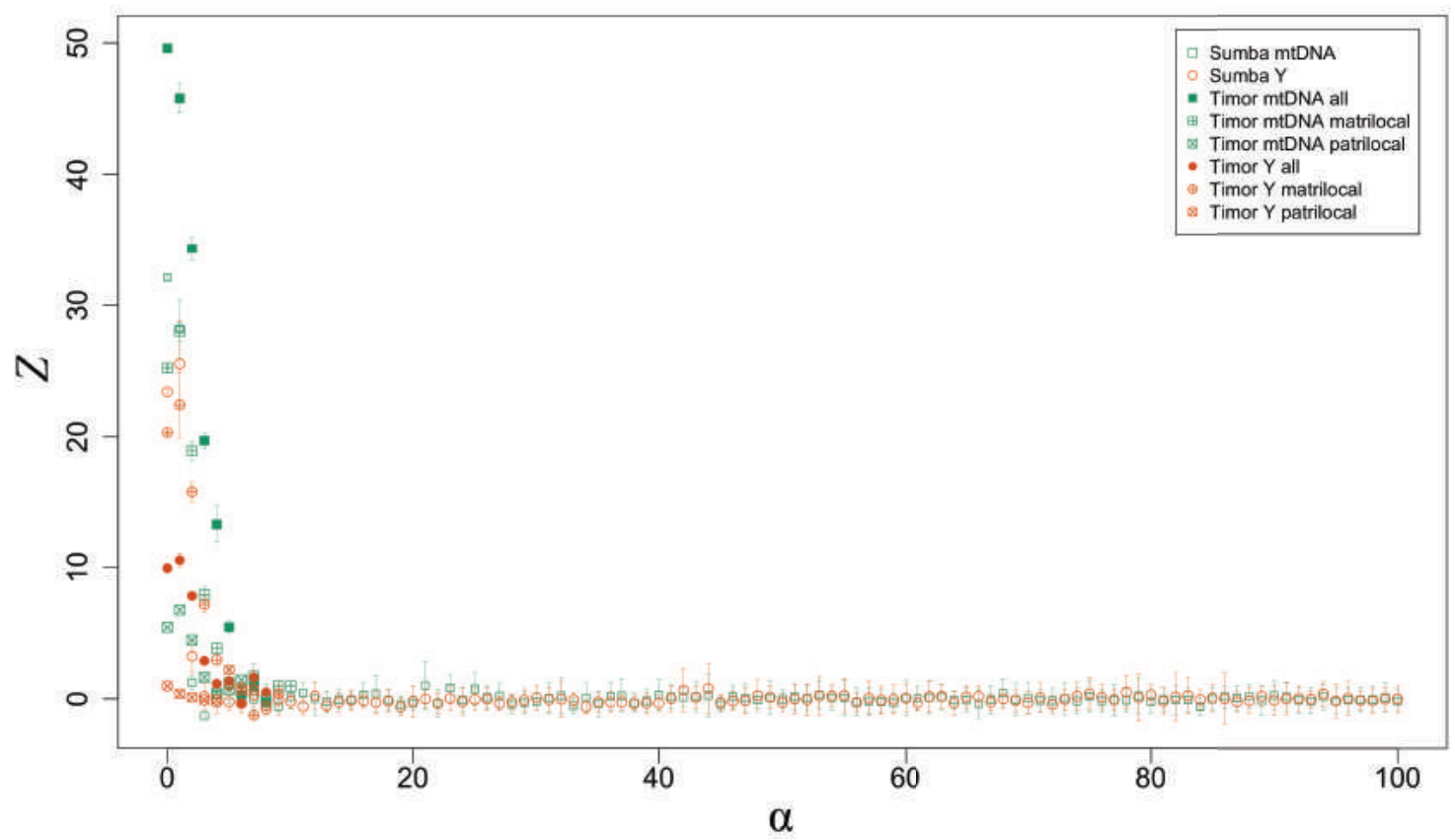

Figure 4.9: Language switching rates from the alternative model.The $Z$ score measure of association between gene and language phylogenies on Sumba and Timor for different language switching rates $\alpha$ in the alternative model. All cases independently converge and abruptly lose gene-language associations, behaving similar to randomized cases, when $\alpha \approx 5 \%$.

\subsection{Summary and Conclusions}

The mtDNA and Y chromosome phylogenies tell us how closely the individuals in our sample are related, but provide no information about how those relationships came to be. But because these individuals were sampled at the community scale, pairwise distance analysis enables us to detect sex-biased migration rates from which we can test inferences about their respective kinship systems.

Language adds a further dimension. For each individual in our sample, we ceon- 
struct two co-phylogenies of language and genetic inheritance, for matrilineal and patrlineal inheritance respectively. If there were no migration between villages, the signal of association in these co-phylogenies would be identical, because men would learn the language spoken by both parents. However, if people sometimes marry into villages where a different language is spoken, the gene and language phylogenies will diverge. If many languages are spoken within a geographical region, and rules of postmarital residence encourage sustained directional population movement between speech communities, then languages should be channeled along uniparental lines. We find strong evidence for this pattern on both Sumba and Timor.

The question of how long language transmission might have persisted along these uniparental lines can be addressed by comparing gene-language co-phylogenies. In each generation, an opportunity exists to weaken or scramble the correlation between genetic and language lineages through host switching, which occurs when children learn a different language than one of their parents. This can lead to three outcomes. In the absence of host switching, the emergence of new languages (branching in the language tree) can introduce persistent clades of related individuals speaking a common language and extensive correlation between the gene and language trees. When host switching occurs rarely, some correlation remains, but frequent host switching and language losses quickly break down down the correlation.

Intriguingly, host switching rates converge to $0.3-1 \%$ per generation for all trees examined here. This translates to a $\sim 50 \%$ probability that a single host switch event will occur within a clade (i.e. "half life" of a language on a lineage) every 1,700-5, 750 years, with near certainty of a host switch event occurring over much longer times frames. This raises the possibility that clades could retain a shared language longer than any single language exists, by replacing one language with another.

Overall, we can infer that low rates of host switching capture the observed genelanguage tree associations. To interpret this result, it is useful to distinguish between social communities and speech communities. With respect to social communities, our results show that kinship rules persist long enough to leave clear traces in population genetic structure. In the 25 communities included in our study, core groups of close relatives must have stayed together for generations, while also remaining in contact with neighboring groups with whom they intermarried. In this way, kinship 
systems directly shaped the language phylogeny over time: consistently following a postmarital residence rule turned social communities into speech communities.

This is particularly clear on Sumba, where each village became a monolingual speech community. Conversely, few villages on Timor are monolingual speech communities; most are multilingual. The likely explanation for multilinguality in central Timor is the political turmoil of the past century, which led to extensive local migration as documented by Therik [62]. It is noteworthy that the association of languages with genetic clades persists on Timor despite these recent population movements.

Overall, the low rates of past host switching are very similar for both islands. This suggests that kin-structured speech communities are quite stable over long time scales. However, we also see genetic evidence for ongoing contact between social communities practicing exogamous matrilocal or patrilocal marriage [63]. In the past, contact between such social communities would often have been synonymous with contact between speech communities.

Previous correlational studies of gene and language trees have focused on the effects of drift, geography (isolation by distance) and large-scale population movements to explain patterns of correlation at different scales of space and time [41, 64, 23]. Our co-phylogenetic analysis at the community scale clarifies how language transmission is actively channeled and continually renewed by kinship practices, creating the observed patterns of language diversity, and leaving a strong signal in population genetic structure. Analysis of gene-language co-phylogenies and pairwise distances suggests that this channeling process usually persists long enough to be regarded as the norm. The Timor data make this point particularly clearly beneath the surface of community scale variation in language diversity created by recent population movements, enduring associations of language with uniparental clades still persist.

Although our focus here has been on the role of kinship in channeling language transmission, clearly language also protects those channels - shared language helps connect and define matrilocal kin in the Wehali region of Timor, just as it connects and defines patrilocal kin on Sumba. Thus, while language and kinship are typically treated as unrelated topics, their dynamic interaction in these villages has been fundamental to the structure of social life. 


\subsection{Future Work}

In the models of language switching on genetic clades, $\beta$ was taken to be the same as $\alpha$, further investigations of the dependence of the $Z$-score of gene-language association on $\beta$ is needed. In conjunction with this, other models of language switching based on linguistic theories can also be explored.

Another possible future study is looking at the general properties of the language switching models. One can test it in simulated gene and language trees wherein the properties of the phylogenies can be controlled. The model can then be ran on these simulated trees to investigate the dependence of co-phylogentic association on various tree properties like for example, size, structure, and depth.

Finally, it is suggested to investigate how a parameter of kinship system can be incorporated in both the Jane method of co-phylogeny reconstruction and on the language switching models. In Jane, the costs of different events can be varied depending on the tree lineage and kinship practice of the system. In the language switching models, the dynamics and rate of host switches can be functions of the kinship practice of the system and which lineage one is looking at (i.e. mtDNA or Y). 


\section{Chapter 5}

\section{Mobility in Transport Networks}

Most people go a short distance while only few go a long distance as described in Ravenstein's seminal work on The Laws of Migration $[65,66]$. Subsequent models of human mobility built upon this idea of universal population displacements creating currents of migration directed towards great centres of commerce, and limited in range by geographic distance $[65,66,67]$. In these works, migration was defined in the inter-country scale for which a geographic definition of distance is sufficient. However, recent works have shown that patterns of mobility vary at different scales $[68,69,70]$. Hence, there's a need to redefine what "distance" is in the context of the system considered. Here, we study movements within the great centres of commerce, that is - distance in the intra-city scale.

An urban city is a locale of high population density marked by intense economic production and consumption. It is a hub of innovation and has been characterized as an engine that drives the economy of nations as well as the world. A city is also a hothouse of socio-economic activities containing a wealth of opportunities and benefits for its dwellers. To partake in these activities, urbanites navigate and traverse actively over the urban landscape through an efficient and robust transport network - a signature behavior observed in most modern cosmopolitan cities.

In a city setting, human mobility is thus characterized by a greater degree of interactions and movements. This should be contrasted with intra- or inter-country travels which lack the travel routine, frequencies, and daily activity-based motivations inherent in intra-city commutes [71]. From this perspective, Ravenstein's laws of migration no longer hold in the environ of urban travels. There is now a need to understand how humans affect and are affected by urban systems. While there has 
been great progress in understanding the individual dynamics of human mobility $[8,6,7]$, much research is still required to understand how our collective movements are constrained by the existing transport infrastructure and how the socio-economic functions of a city are served through intense urban travels.

To gain and advance our understanding on human mobility in such an urban setting, the development of theoretical models is necessary and crucial. Currently, there are models that are specifically created to address human movements within certain cities-of-interest $[72,73,74,71,75]$. Our goal, however, is to develop models that are general, that bear the core essence of human mobility, and can be tailored to encompass the traits of urban travels. Conventional models in this context are the gravity model and the model of intervening opportunities, which are dependent on empirical parameters and are not supported by a rigorous underlying theoretical framework. On the other hand, the recent introduction of a new radiation model has put the purposefulness of human mobility into the theoretical construct, and this model embodies fundamental features that were built from first principles [7]. Interestingly, in contrast to the standard models, the radiation model exhibits an average commuting flux that is not directly dependent on distance. It also has the unique quality of being parameter-free. The resulting emphasis on topological instead of metrical properties makes it directly applicable to the modelling of intracity travel, as such commutes are typically driven by commuters wanting to satisfy a functional need where distance is no longer the main concern in its travel decision.

However, it was found by Masucci et al. [76] that these models are still inadequate to model human mobility at the city scale. This conclusion is supported by a host of other studies $[77,78,79,80,81,82,83]$ which ascribe the deficiency to be a consequence of the universality of the radiation model. Real-world cities have heterogeneous properties and contain spatial-scales that are absent in the assumptions of the radiation model, which explain why it has not been successful in accounting for the patterns in real-data. To overcome these issues, researchers have either modified the model or introduced an alternative model. The former has led to a generalized radiation model [83], which makes improvement by including adjustable parameters into the radiation model. The latter (population-weighted opportunities model) modifies the assumptions of the radiation model by enlarging the area of selection for the benefit region and by incorporating a measure of attractiveness to 
the destination of travel [81] to compensate for the radiation model underestimating the mobility flux.

We propose a modified radiation model for urban travels which is derived from the same first principles used in the original radiation model and retains its independence to tuning parameters, but makes it applicable to high intensity, high frequency and activity-based commutes within cities. In this model, we redefine the distance assumption by considering the heterogeneities in the topology of the system. We consider topology instead of metrical distance by incorporating (1) land use pattern and (2) the transport network as parameters in a commuter's travel decision. We also redefine the job selection benefit parameter in the original radiation model (previously quantified by population density) by factoring in the purpose of travel and looking at activity-based commutes. This is achieved by examining the heterogeneity in the use of space as described by a land use plan and the corresponding density of amenities.

In the following section, we describe briefly the details of the conventional models of mobility: (1) gravity model, (2) model of intervening opportunities, and (3) original radiation model. We then proceed with describing how we modified the radiation model to improve predictions of urban travels. We apply this model to the case of Singapore where information on land use pattern, planned amenities density, transport structure and actual daily transport network use are available.

\subsection{Conventional models of human mobility}

In the context of travels, the purpose of human mobility models is to create a trip distribution that splits a total number of trips $N$ into a trip table $T \equiv\left(T_{i j}\right)_{i \neq j}$, that estimates the number of trips from one area to all other [84]. Trip distribution modelling is crucial in predicting travels and understanding the mechanisms of human mobility [84]. We discuss below the conventional models used to create a probability trip distribution at an aggregate level.

\subsubsection{Gravity model}

Perhaps the most traditional approach in estimating trip distributions is the gravity model. It was first suggested for use in human mobility by Carey in 1859 [85] and was 
later popularized by Zipf in 1946 [67]. It has since then been the most widely used model of mobility with extensive applications in flows of population, accessibility to health services, international trade, traffic and communications [84].

In analogy with Newton's law of gravity, the gravity model of mobility assumes that the amount of trips between two locations $i$ and $j$ is proportional to the product of "mass" parameters, which here is the origin population $m_{i}$ and destination population $n_{j}$, and inversely proportional to a power law of the distance travel cost $r_{i j}$ between them $[76,84]$. Using this version of the gravity model, the trip distribution can then be estimated as

$$
T_{i j}=T_{i} \frac{m_{i} n_{j}}{r_{i j}^{\gamma}},
$$

where $T_{i}$ is a normalization factor and $\gamma$ is the only parameter determined by regression analysis.

\subsubsection{Intervening opportunities model}

Stouffer proposed in 1940 the alternative model of intervening opportunities [66] which hinges on the assumption that more than distance, the number of opportunities plays an important role in destination decisions. The original version proposed by Stouffer was later reformulated by Scheider in 1959 [86] to its current widely used form.

The intervening opportunities model stresses that trip making is only indirectly related to distance and it is instead controlled by the relative accessibilty of opportunities that will satisfy the purpose of the trip [81]. In Schneider's formulation of the intervening opportunities model, the probability of travelling from location $i$ to $j$ is proportional to the origin population $m_{i}$, and to the conditional probability that a person living in origin $i$ is attracted to destination $j$ with population $n_{j}$ given that there are $s_{i j}$ opportunities between the two locations [84]. The trip distribution is then given by

$$
T_{i j}=T_{i} \frac{e^{-\alpha\left(s_{i j}-n_{j}\right)}-e^{-\alpha s_{i j}}}{1-e^{-\alpha M}},
$$

where $s_{i j}$ is the total population (including populations in $i$ and $j$ ) in a circle of radius $r_{i j}$ centered in $i, M$ is the total population in the considered area, and $\alpha$ is 
the probability of being satisfied with the opportunities in $j$.

\subsubsection{Original radiation model}

Recently, Simini et al. [7] further reformulated Stouffer's intervening opportunities model, based on a first principles derivation in terms of radiation and absorption processes. It follows from a simple particle diffusion model, where particles (i.e. individuals) emitted from location $i$ have a probability $p$ of being absorbed by location $j$ depending on the spatial distribution of intervening opportunities. The probability for a particle to be absorbed was found to be independent of the distribution of proability $p$.

The trip distribution predicted by this model is multinomial with average travels between $<T_{i j}>$ dependent on the origin population $m_{i}$, the destination population $n_{j}$, and on the population $s_{i j}$ within a circle with radius $r_{i j}$ and centered in $i$ (excluding the origin and destination populations), that is

$$
<T_{i j}>=T_{i} \frac{m_{i} n_{j}}{\left(m_{i}+s_{i j}\right)\left(m_{i}+n_{j}+s_{i j}\right)} .
$$

Unlike the gravity and intervening opportunities models, the radiation model is parameter-free and directly computable from census data.

\subsection{Radiation model modified for urban travel}

We show here how we modified the radiation model for it to be applicable to human movements in the urban scale. To achieve this, we have enhanced the radiation model with four characteristics that serve to extend or revise its basic assumptions. These four characteristics are: (1) urban land-use, (2) opportunities density, (3) boundary porosity, and (3) transport network structure. We first discuss the system we used to test our enhanced radiation model framework. We then go into detail on each of these characteristics before finally presenting the modified radiation model.

\subsubsection{Rapid Transit System}

The urban transport system is a network of the different modes of transportation (i.e. bus, tram, Rapid Transit System). Of these, the Rapid Transit System (RTS) 


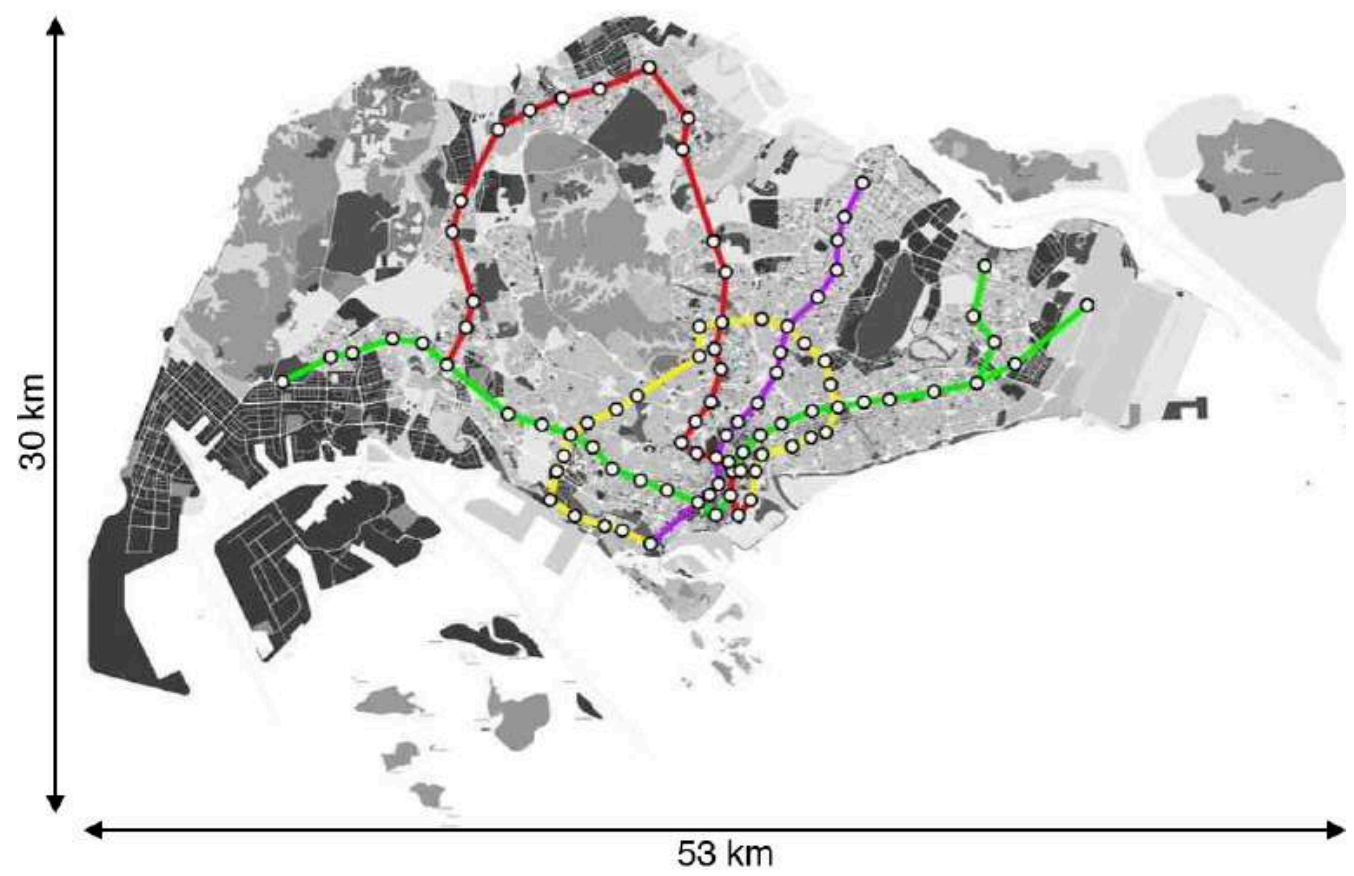

Figure 5.1: Singapore Mass Rapid Transit. 2008 urban planning map of Singapore with four MRT lines (Red - North South Line, Green - East West Line, Purple North East Line, Yellow - Circle Line) having a total of 90 stations and 97 links.

is becoming the most utilized mode of public transportation because of its high capacity, high frequency and grade separation from other traffic [6]. The increasing use of RTS makes it a key indication of urbanization.

As an initial case sample for developing a spatio-temporal model of mobility in transport networks, we concentrate on Singapore's Mass Rapid Transit (MRT) system shown in Fig. 5.1. The model developed for the case of Singapore's MRT can be extended or applied to other transport networks and to a network of different transport modes.

Currently, Singapore's MRT serves around 40\% of the country's population and hence it's significance in defining movements within the country cannot be undermined. Singapore's MRT network in the 2008 Master Plan is composed of four MRT line networks with 90 nodes or stations and 97 links or station connections. 


\subsubsection{Characteristic parameters of the radiation model mod- ified for urban travel}

The original radiation model was formulated in the context of inter-country travels. Upon application to intra-city travels, many have found it to be insufficient. We now show the characteristic parameters that we include in the radiation model for it to be suitable for predicting urban travels.

\subsubsection{Urban land-use}

A person's movement in a city is influenced by the function of the specific area that defines a region. When an individual decides where to live and where to travel or commute everyday, the first consideration is where the different land-use sectors are located. Initially, 13 land-use entities were considered. We then tested our model on all $n$ combinations of these 13 land-use entities, where $1 \leqslant n \leqslant 13$. We present here the six land-use entities that yielded trip distributions that have the highest correlation with actual trip distributions in Singapore's MRT.

In our enhanced model, we have considered the following land-use entities: business, commercial, educational, hotel, medical care and residential (Fig. 5.2). To simplify our investigation, we have categorized business, commercial, educational, hotel and medical care together as one land-use unit that we term "work", with the remaining residential entity being called "home".

\subsubsection{Opportunities density}

The relative importance and usage of the different types of land-use sectors can be estimated by looking at the Master Plan drawn up by the governing authority of the city. The Master Plan indicates the gross-plot-ratio (GPR) allotted to all parcels of land. Specifically, GPR is a measure of the gross floor area per site area, and is used as an estimate of an area's carrying capacity. Fig. 5.3 shows the locations in the urban planning map of Singapore with corresponding GPR measures for the different land use entities considered [87]. The URA Master Plan did not include GPR values for educational institutions and hence for our purposes we assumed each educational institution pixel to have $G P R=1$.

In addition, the Master Plan also illustrates the morphology of the amenities in 


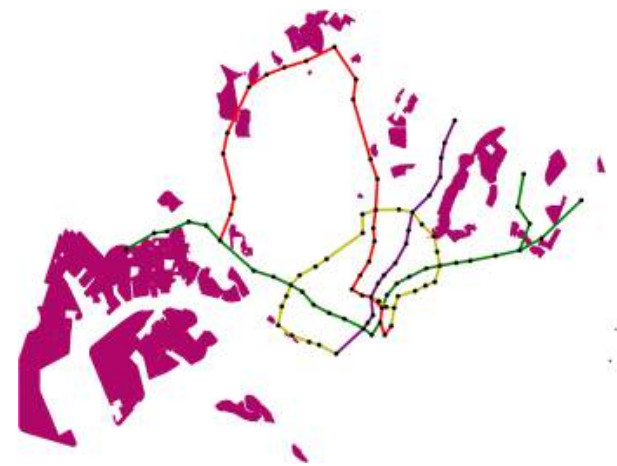

(a) business

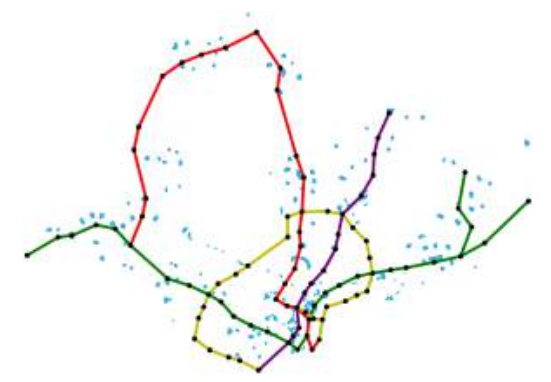

(c) commercial and residential

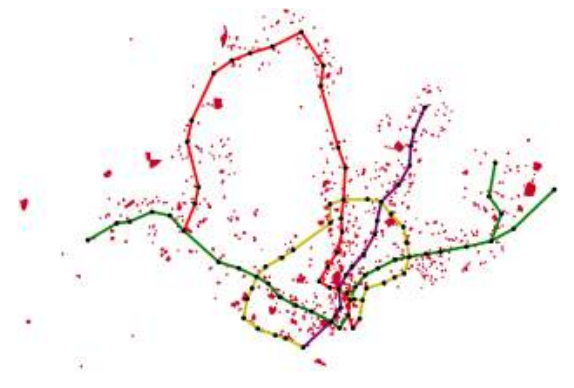

(e) health and medical care

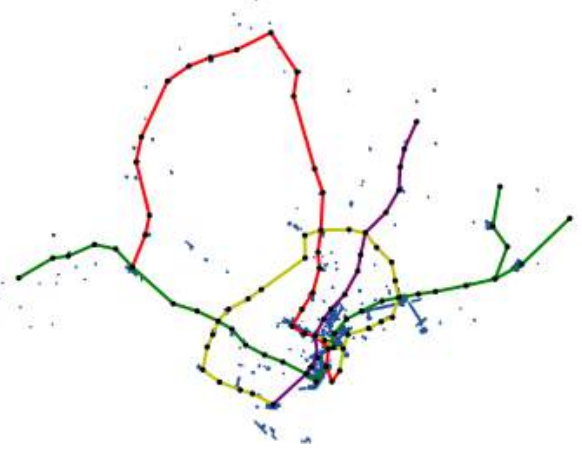

(b) commercial

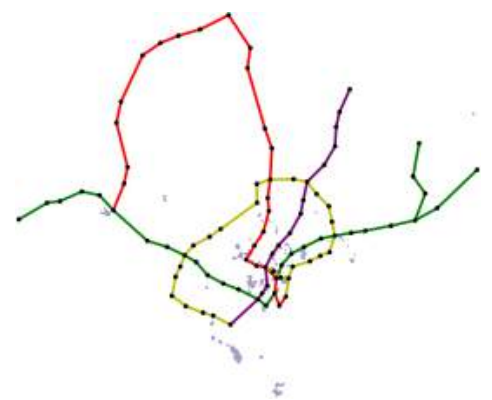

(d) hotel

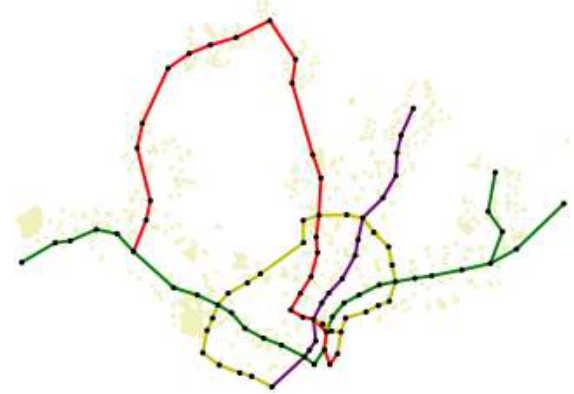

(f) educational institution

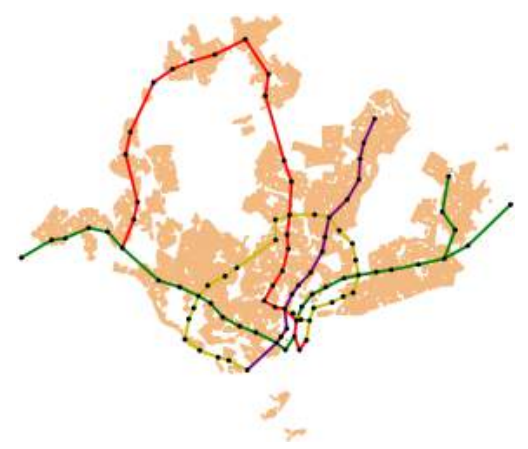

(g) residential

Figure 5.2: Land use entities. The defining mobility features in Singapore Rapid Transit System (RTS). (a) Business, (b) commercial, (c) commercial and residential, (d) hotel, (e) health and medical care, and (f) educational institution are considered "work" entities while (g) residential entities are considered "home" locations. 


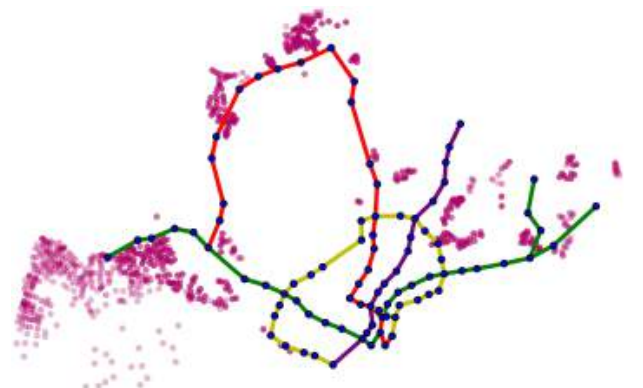

(a) business

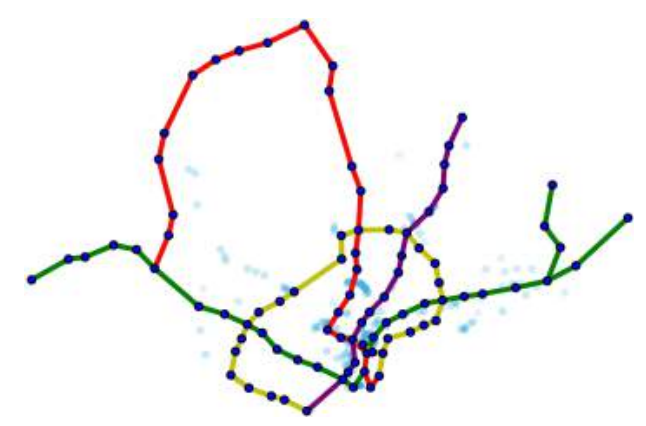

(c) commercial and residential

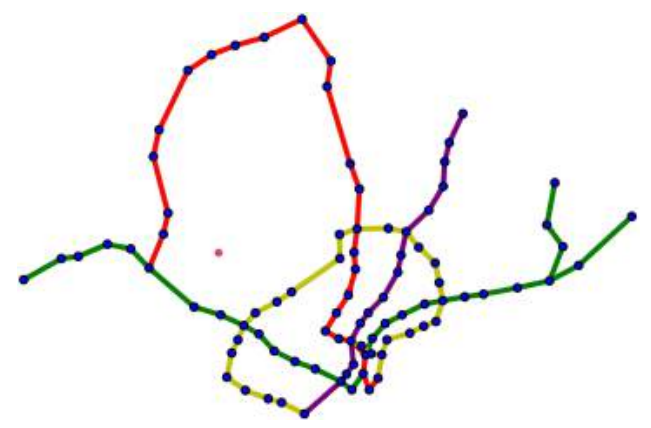

(e) health and medical care

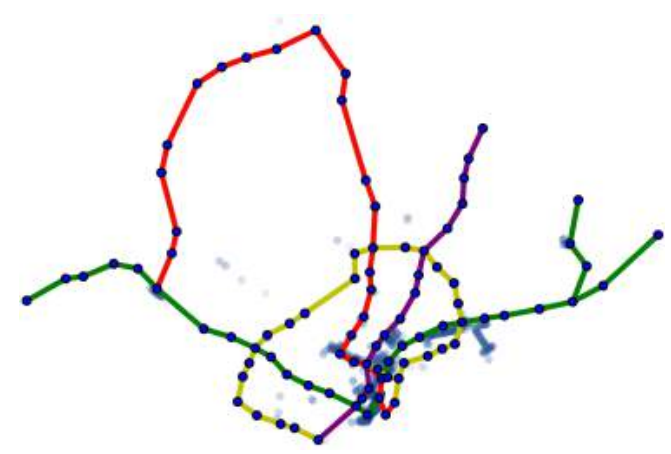

(b) commercial

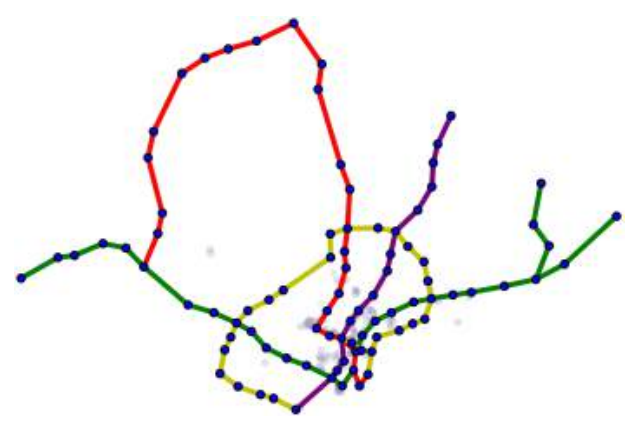

(d) hotel

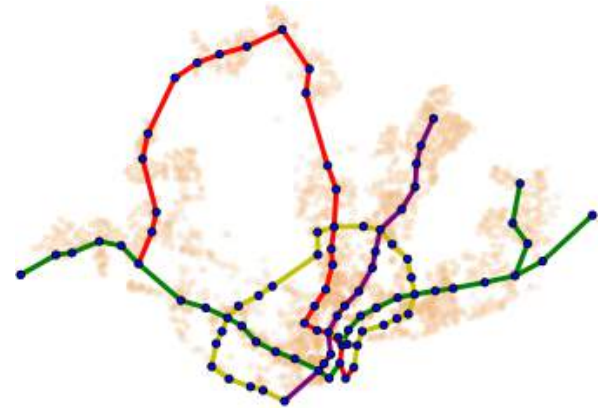

(f) residential

Figure 5.3: Gross plot ratio for the land use entities. Points refer to the locations in the map for which a GPR measure is available for each of the land use entities considered. 
the city, which we quantify by means of a spatial entropy measure. This measure characterizes the amenities according to whether they are scattered or agglomerated, and it serves to evaluate the attractiveness of the locale under study [88, 89]. For a given land-use type $T$, the spatial entropy $E_{T}$ of a land-use Master Plan map $M$ with $k$ frames is given by

$$
E_{T}(M)=-\sum_{k}\left[p_{k} \ln \left(p_{k}\right)+\left(1-p_{k}\right) \ln \left(1-p_{k}\right)\right]
$$

where the density of pixels in a frame $p_{k} \equiv t / N_{k}$ with $t$ being the number of pixels of type $T$ and $N_{k}$ being the $l x l$ pixels in frame $k$. Here, we considered a frame size of $256 x 256$ pixels with each pixel corresponding to a land area of $64 \mathrm{~m}^{2}$. Eq. 5.4 is normalized as

$$
\hat{E}_{T}(M)=\frac{E_{T}(M)}{E_{T}\left(M^{\prime}\right)},
$$

where $M^{\prime}$ is a copy of $M$ with type $T$ pixels having the same density but distributed randomly in the map area.

To determine the density of commuters $g_{T}$ in the area due to the strength of opportunities available from either of the "work" or "home" land-use entity $T$, we multiply the sum of GPR of the corresponding entity with $\left(1-\hat{E}_{T}(M)\right)$ entropy measure of the area-of-interest.

\subsubsection{Boundary porosity}

In the urban landscape, the function of a city region is coupled to its transport network. We model this coupling by relating the land-use map to the Rapid Transit System (RTS) of the city. This is achieved by carefully prescribing the city region most likely to be served by each of the station node of the RTS. We give two perspectives to this prescription.

The first perspective is the Voronoi region, $V_{i}$, for station $i$ of the RTS. Note that a Vorono $i$ tesselation is constructed with each RTS stations located at the seed points $\left\{z_{i}\right\}$. With these set of points, we limit each Voronoi site to contain one and only one station. In other words, all the points in each of the sites are closest to the station on it than any other stations located at the other sites. That is, a point $q$ 


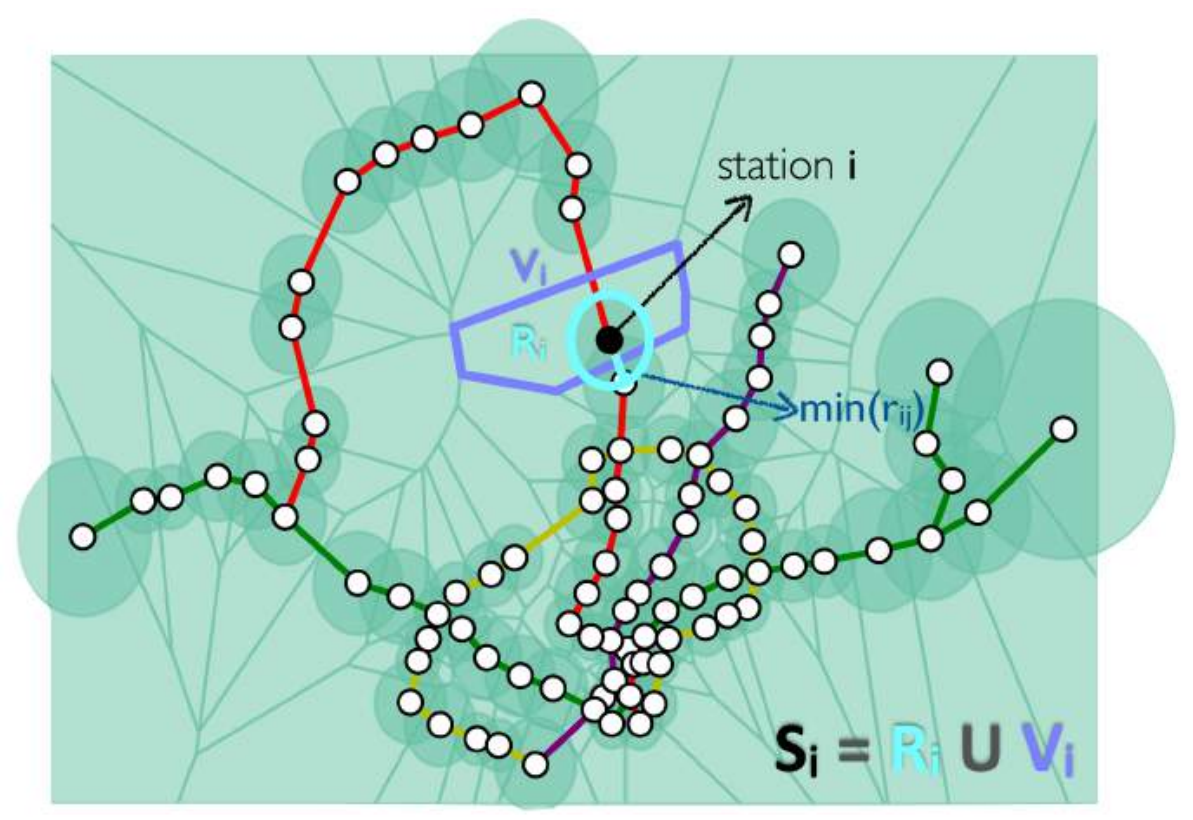

Figure 5.4: Singapore MRT voronoi regions of influence. Each station serves a region of commuters as indicated above.

in site $i$ should obey the following condition:

$$
\left\|q-z_{i}\right\|<\left\|q-z_{j}\right\| \text { for } j \neq i
$$

The second perspective defines a region $R_{i}$ for station $i$ of the RTS. $R_{i}$ is the set of points situated at a distance at most $\min \left(r_{i j}\right)$ away from station $i$, where $r_{i j}$ is the distance between station $i$ and station $j$. That is, $\min \left(r_{i j}\right)$ is the minimum distance between station $i$ and the set of nearest stations $\{j\}$ that are connected to it.

Putting these perspectives together, we define the city region served by station $i$ to be the region $S_{i}$ which is made up of the set of points $q$ in $R_{i} \cup V_{i}$. Specifically, $S_{i}$ is given by

$$
\left\|q-z_{i}\right\| \leqslant \min \left(r_{i j}\right) \cup\left\|q-z_{i}\right\|<\left\|q-z_{j}\right\| \text { for } j \neq i .
$$

This condition gives rise to the porosity of the boundary in the modified radiation model since the original radiation model operates based on a spatially discretized settlement structure. The possibility that $S_{i}$ and $S_{j}$ (with $i \neq j$ ) overlaps reduce the overall spatial discrete structures within the model and thus provide a more continuum approach that improves the generality of its modeling capacity. 


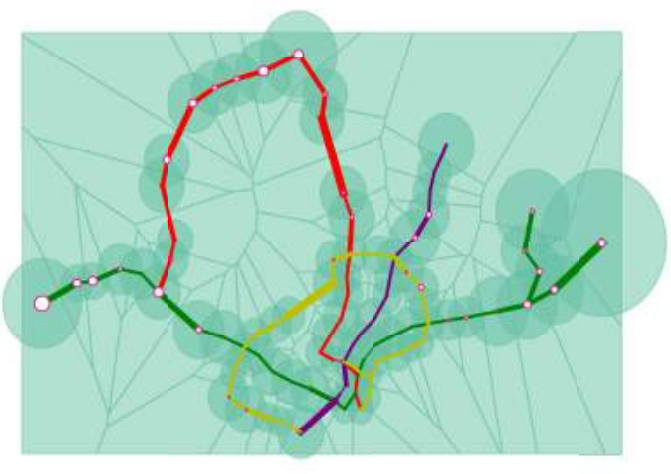

(a) business

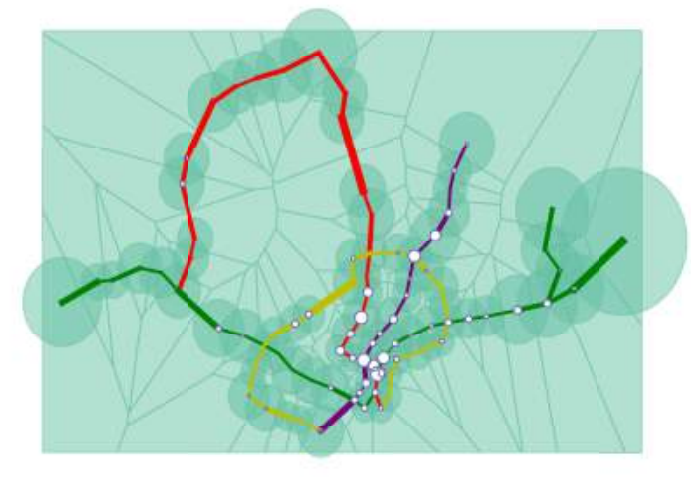

(c) commercial and residential

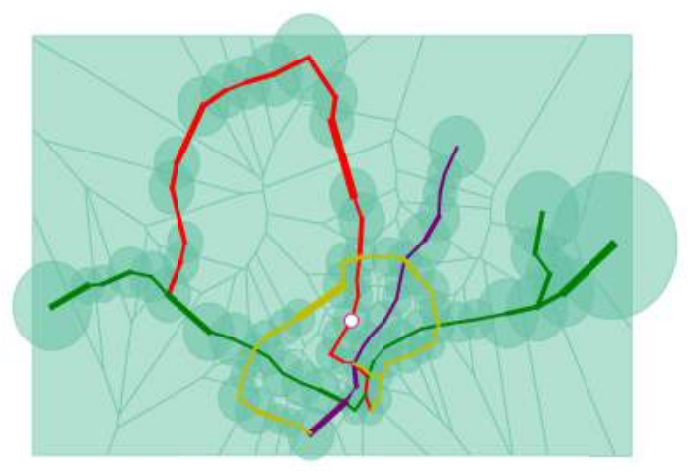

(e) health and medical care

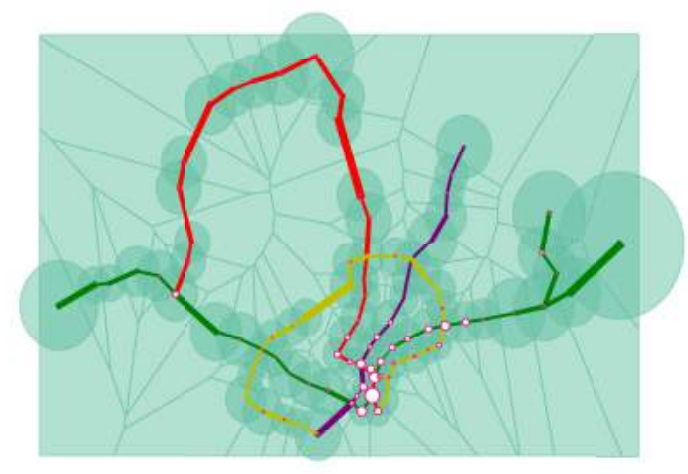

(b) commercial

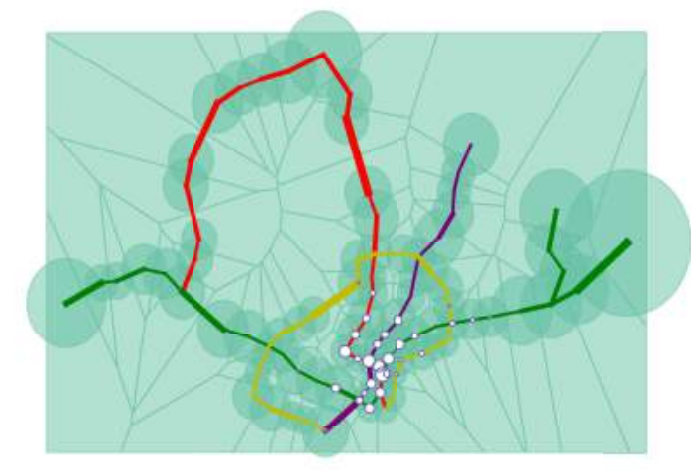

(d) hotel

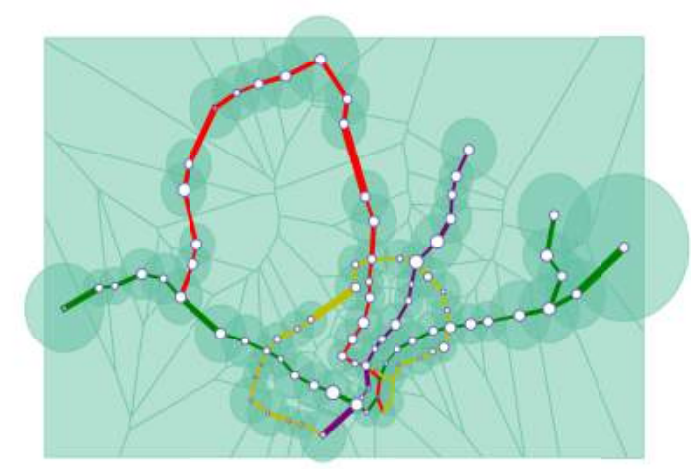

(f) residential

Figure 5.5: Singapore RTS network weighted by density estimates for each land use entities. Network node sizes are weighted according to normalized sum of GPR values for each of the considered land use types. Network is superimposed over the Voronoi tesselation. 


\subsubsection{Transport network structure}

Human travels in a city is enabled by different modes of transportation consisting of RTS, trains, trams, buses, and cars. In particular, each of this system can be described by a mathematical network whose structure has important effects on the flow of human traffic through it. Taking the RTS as an example, stations that are more central are more likely to be part of a commuter's route. We have tested different centrality measures and found node degree centrality of the transport network weighted by the travel time between stations (which is equivalent to the degree of a node) to be the most suitable measure of the station's travel demand. A station's node degree centrality $C_{i}$ is given by

$$
C_{i}=\sum_{j=1}^{N_{s}} A_{i j}
$$

where $N_{s}$ is the total number of stations and $A_{i j}$ is the adjacency matrix of the weighted transport network detailing the travel times between pairs of stations $i$ and $j$. Fig. 5.6 shows the results when only the node centrality measures were correlated with the data.

\subsubsection{Enhanced radiation model}

Incorporating these enhanced characteristics into the standard paradigm of the radiation model, the average number of commuters traveling from regions $S_{i}$ to $S_{j}$ with the origin region being $O$ and the destination region being $D$ becomes

$$
\left\langle T_{i j}\right\rangle^{O D}=\left(g_{i}^{O} N_{c}\right) \frac{g_{i}^{O} g_{j}^{D}}{\left(g_{i}^{O}+s_{i j}^{D}\right)\left(g_{i}^{O}+g_{j}^{D}+s_{i j}^{D}\right)},
$$

where $O$ and $D$ designate either "home" or "work". Note that

$$
g_{i}^{O}=C_{i} \sum_{z_{O}=1}^{N_{i}^{O}} \operatorname{GPR}_{z_{O}}^{i}\left(1-\hat{E}_{O}\right),
$$

and $g_{i}^{D}$ is similarly defined. $\mathrm{GPR}_{z_{O}}^{i}$ represents the GPR of type $O$ in region $S_{i}$ at site $z_{O}$. In other words, there is a total of $N_{i}^{O}$ sites of type $O$ in $S_{i}$ with each site being enumerated by $z_{O}$. The term $C_{i}$ denotes the node degree centrality of station

$i$. $\hat{E}_{O}$ is an entropy measure that quantifies the spatial organization of amenities of type $O$ in region $S_{i}$. Next, $s_{i j}^{D}$ signifies the competition for commuters between a 


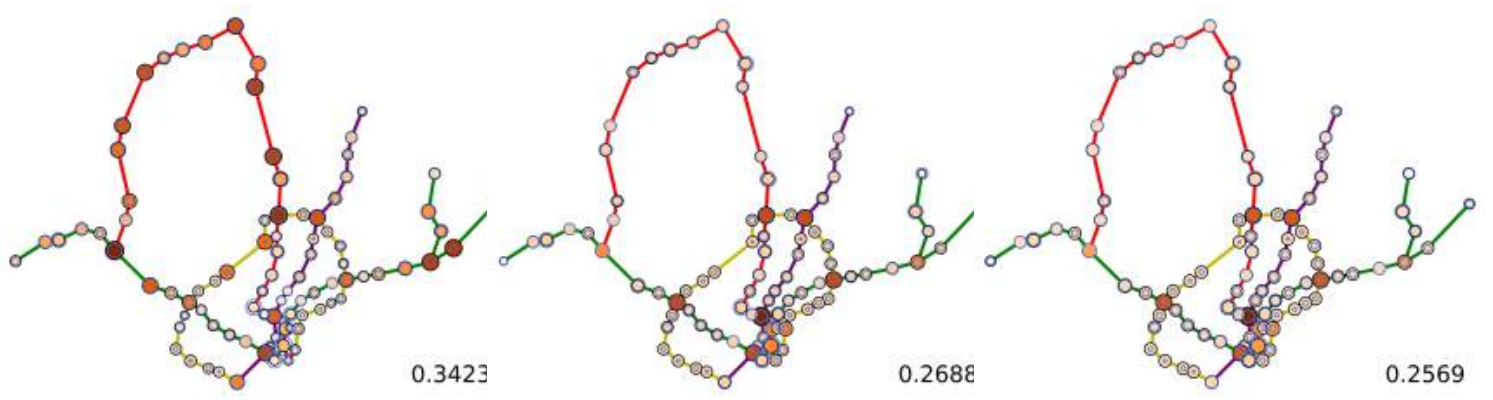

(a) degree

(b) number of cliques

(c) communicability

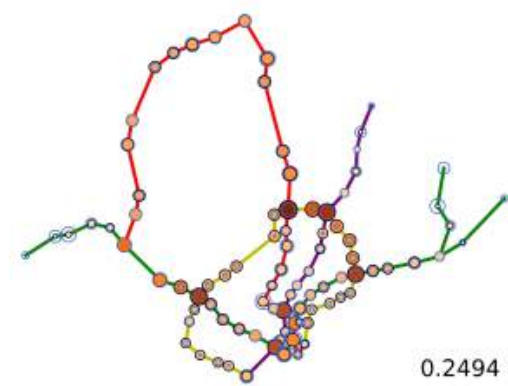

(d) current flow betweenness

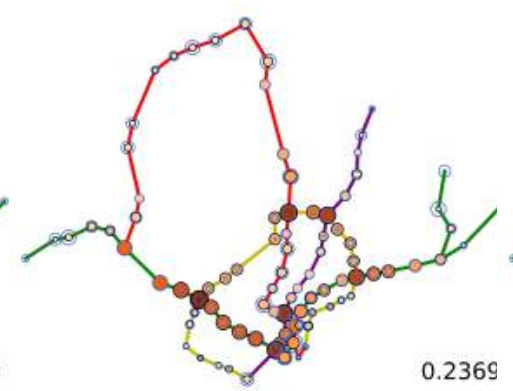

(e) betweenness

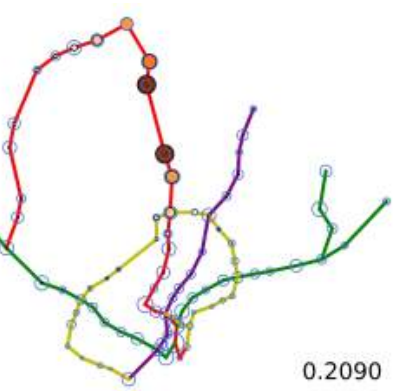

(f) eigenvector

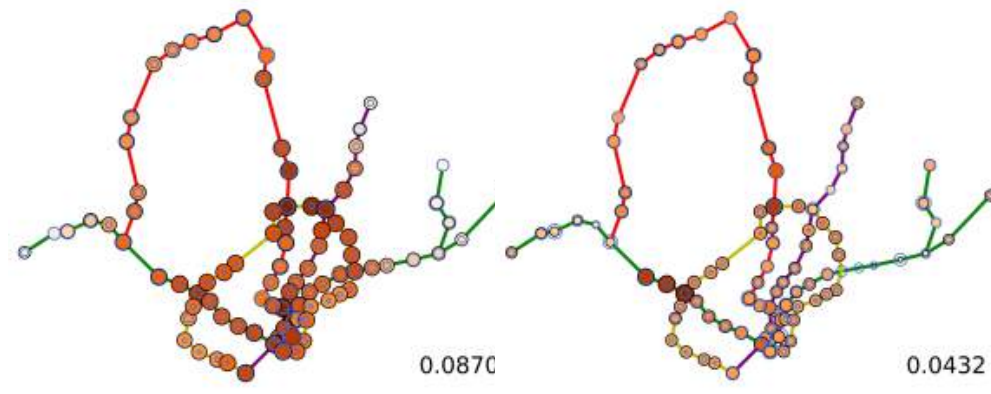

(g) current flow closeness

(h) closeness vitality

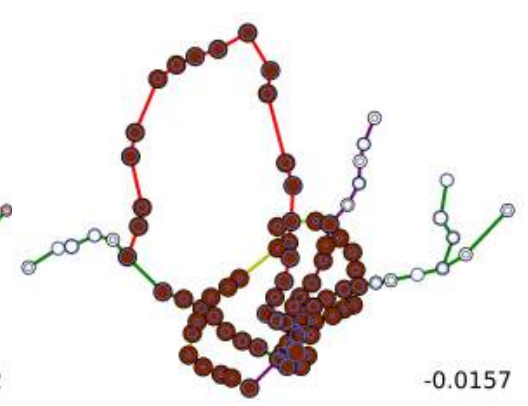

(i) core number

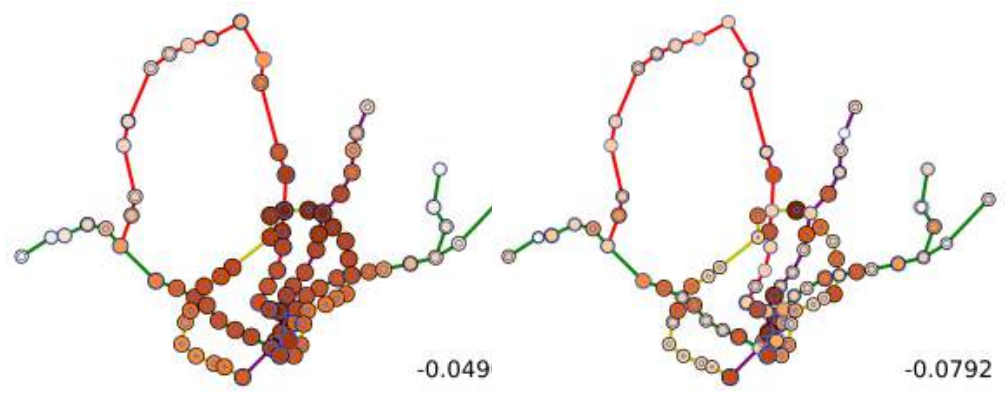

(j) closeness

(k) average neighbor degree

Figure 5.6: Correlation of RTS network node centrality measures with data. The bigger and darker the network nodes are, the higher the node centrality measures. The unfilled blue circles are mean of total tapins in the stations per day over a five weekday period. 


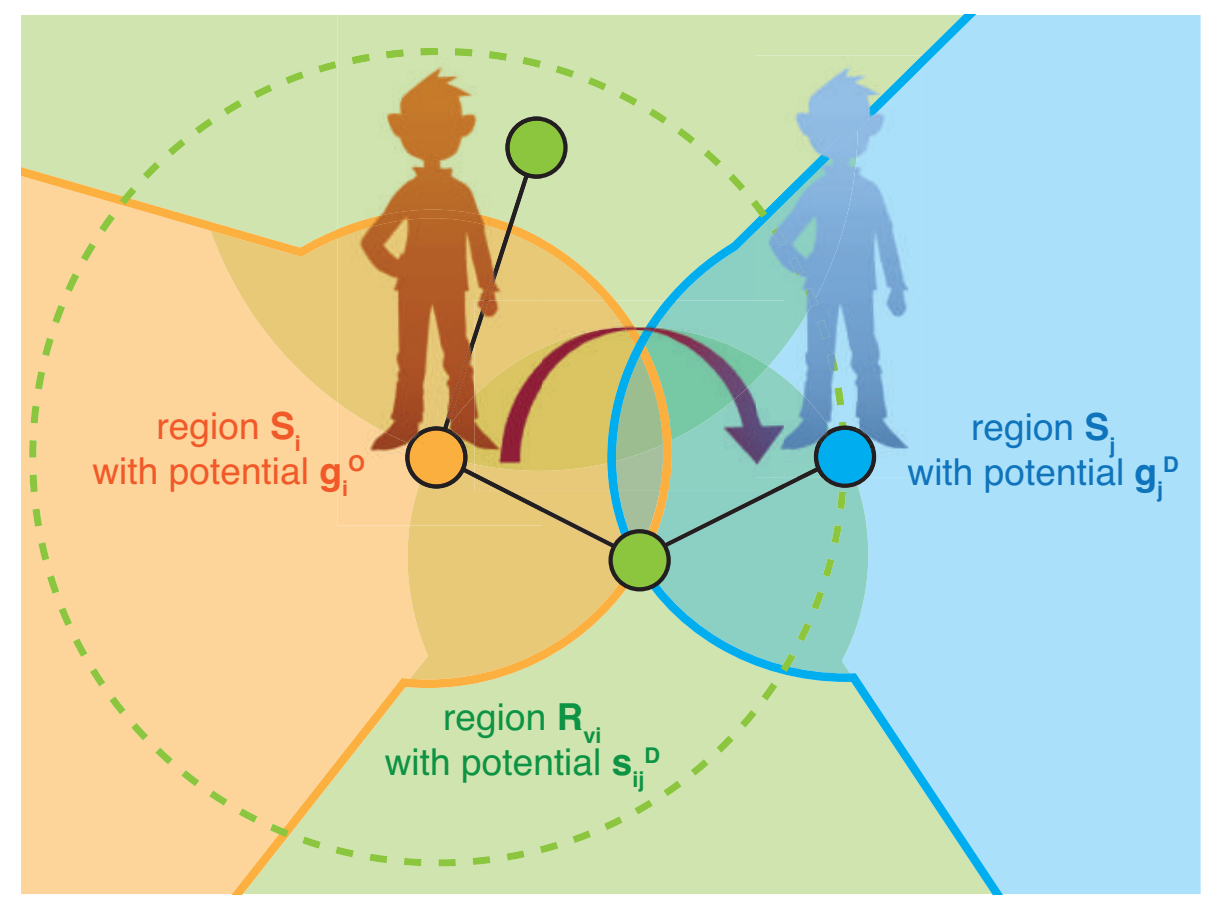

Figure 5.7: Modified Radiation Model. A commuter in region $S_{i}$ with absorption threshold dependent on potential $g_{i}^{O}$ decides to travel to region $S_{j}$ with absorbance dependent on potential $g_{j}^{D}$ given the intervening absorbances dependent on potential $s_{i j}^{D}$ in region $R_{v_{i}}$.

region $R_{v_{i}}$ and the destination region $S_{j}$ according to the original radiation model. Note that $R_{v_{i}}$ is a circular region of radius $r_{i j}$ centered at station $i$. Formally,

$$
s_{i j}^{D}=C_{v_{i}} \sum_{z_{D}=1}^{N_{v_{i}}^{D}} \operatorname{GPR}_{z_{D}}^{v_{i}}\left(1-\hat{E}_{D}\right),
$$

where $\mathrm{GPR}_{z_{D}}^{v_{i}}$ is the GPR of type $D$ in region $R_{v_{i}}$ at site $z_{D} . N_{v_{i}}^{D}$ is the total number of type $D$ sites in $R_{v_{i}}$. On the other hand,

$$
C_{v_{i}}=\sum_{z=1}^{N_{v_{i}}^{s}} C_{z}
$$

is a sum of node degree centrality $C_{z}$ of the $N_{v_{i}}^{s}$ RTS's stations that are located within $R_{v_{i}}$. Furthermore, $\hat{E}_{D}$ is the entropy measure of amenities of type D as before. Finally, $N_{c}$ is the total number of commuters in the system.

In summary, the modified radiation model requires explicit specification of landuse within $S_{i}$ during the calculation of the mobility flux $\left\langle T_{i j}\right\rangle^{O D}$. Like the original model, $S_{i}$ radiates or absorbs a commuter based on $g_{i}$. However, unlike it, $g_{i}$ now 
depends on both the population density and the transport network structure. The original radiation model is known to provide a universal method [7] to determine the number of journeys made between locations. A criticism on its validity concerns its failure to capture the diversity in motivations on human mobility. In addition, it neglects segregation that originates from social-geographical and social-economic factors which have important influence on the flow of commuters. With land-use as a proxy to the purpose of commute, the modified model is now able to properly account for the heterogeneity of travel demands. Moreover, there is a suggestion that amenities density is a more appropriate variable than population as a key driver of human mobility [76]. Here, we have used GPR weighted by entropy as a measure of opportunities density to indicate the affinity of a region as a source and a sink of travel. Since travel is heavily dependent on infrastructures such as the RTS in an urban area, this affinity needs to be further weighted by the "attractiveness" of the station of the RTS located in the region-of-interest. This attractiveness is duly captured by the node degree centrality of the RTS network.

\subsection{Comparison of results from the modified ra- diation model with real-data from Singapore RTS}

In this section, we shall apply the Modified Radiation Model (MRM) to predict the travel demands of commuters riding the Singapore RTS which is better known as the Singapore Mass Rapid Transit (MRT) system. The Singapore MRT system currently serves around $40 \%$ of the country's population and it is the dominant mode of transport in the country. At the moment, it is composed of five MRT lines with a total of 106 stations. Our investigations, however, are on the MRT system of 2013 which at that point in time possesses four MRT lines, 90 station nodes, and 97 links between stations as shown in Fig. 5.1.

In modeling the land-use of Singapore, we extract from the Urban Redevelopment Authority's 2008 Master Plan [87] the gross plot ratio of the land parcels in Singapore. Initially, thirteen land-use entities were considered. From simulations performed, mobility in transport networks is best described by the six land-use 


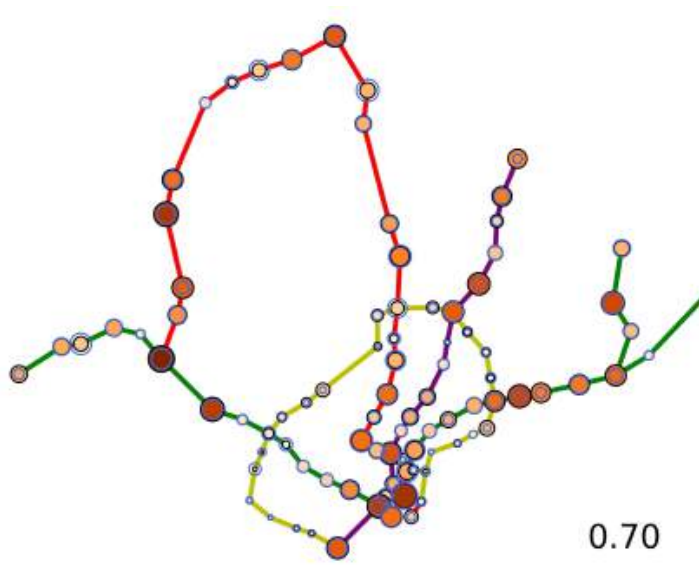

(a)

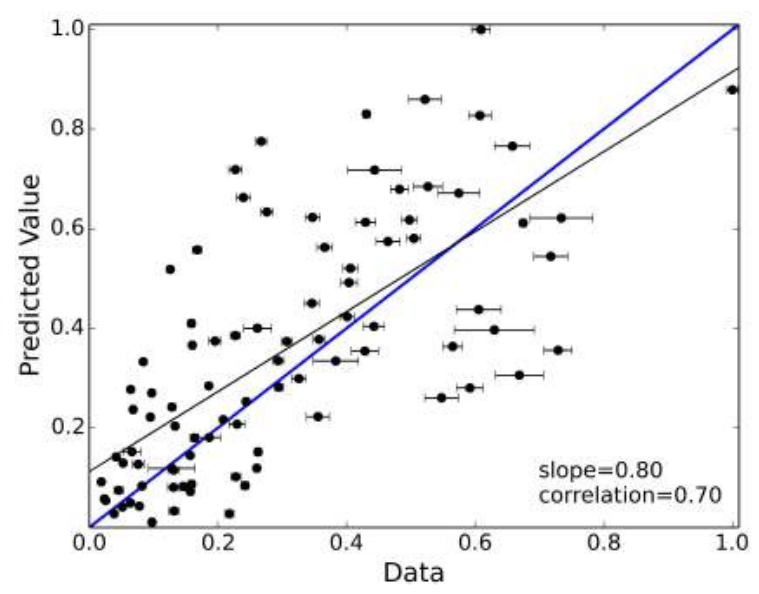

(b)

Figure 5.8: Travel demand prediction. Predicted values have correlation of 0.70 with data. Filled circles in (a) represent predicted values while unfilled blue circles are from the data.

entities: business, commercial, hotel, health and medical care, educational and residential (Fig. 5.2). The results are robust as no significant difference is observed by simulating an additional land-use unit. Fig. 5.3 shows the locations in the urban planning map of Singapore with corresponding GPR measures for the different land-use entities considered.

All human movements require an origin and a destination. In describing the journeys made in the MRT network, we need to consider the typical origins and destinations of commuters. We limit our study to journeys carried out on weekdays as travel patterns on weekdays are more regular. During weekdays, most journeys are: home to work or school in the morning; and work or school to home in the afternoon. There are around 2.3 million journeys made every weekday in Singapore's MRT [6]. From this, we assume that there are 1.1 million home to work or school journeys in the morning; and there are 1.2 million work or school to home journeys in the afternoon. Residential entities are assigned as the "home" location. On the other hand, business, commercial, hotel, health and medical care, and educational institution entities are assigned as the "work" location.

Using $G P R$ values weighted by both entropy measure and node degree centrality as the potential measure in the radiation model yielded travel demand estimates that has a correlation of 0.70 with data (Fig. 5.8). For comparison, we also calculated the 

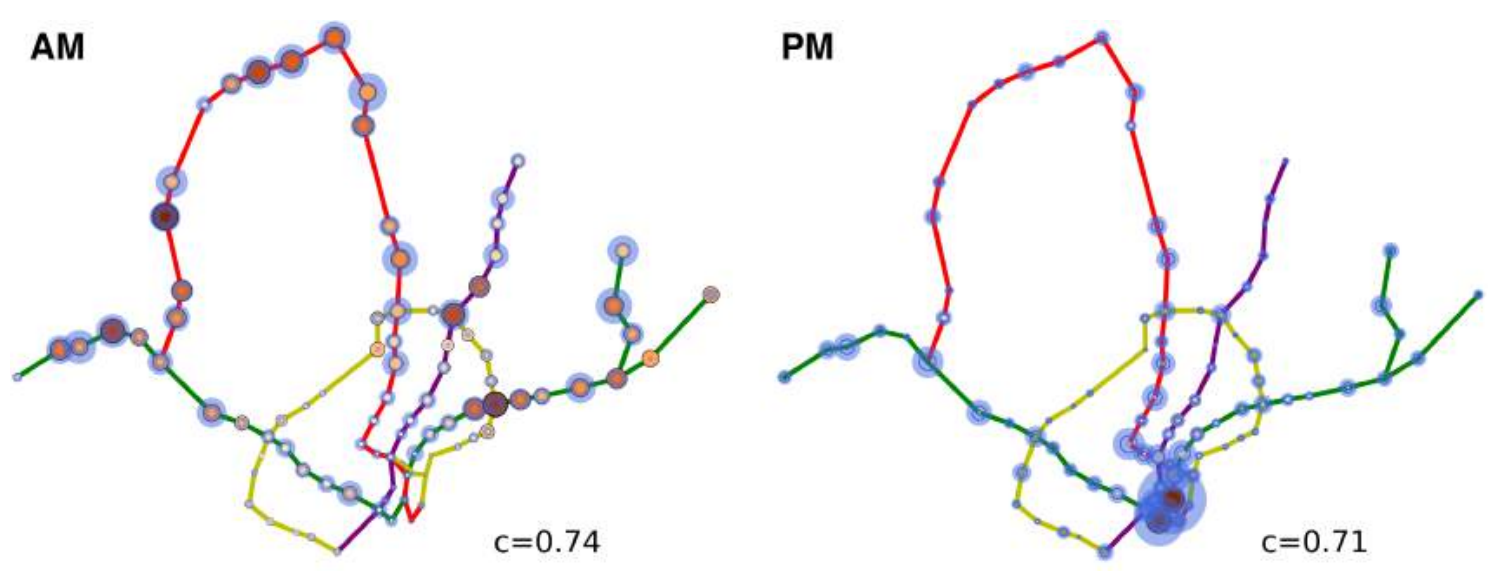

Figure 5.9: Travel demand prediction for morning and afternoon rides. Prediction for morning trips has a correlation of 0.74 with the data while that for afternoon trips has 0.71 . The blue regions represent the predicted mean and standard deviation of travel demand.

correlation for the gravity model and for the original radiation model. Both models with correlations 0.54 and 0.60 respectively, made less powerful predictions as compared to the model presented here. Considering the morning (AM) and afternoon (PM) trips separately, we see that the the morning trips are better predicted by the model. The modified radiation model for urban travels has a correlation of 0.74 for the morning trips and 0.71 for the afternoon trips (Fig. 5.9). This might be due to the fact that commuters are more likely to deviate from their usual route in the afternoons after work.

When correlation was calculated for each MRT line (Fig. 5.10), the Circle line had the highest correlation while the North-South line had the lowest. Further, when we look at the slope $(m)$ of trend lines, we notice two groups, group (1) with the Circle and North-East lines having $m=1.33 \pm 0.05$ and group (2) with the EastWest and North-South lines having $m=0.69 \pm 0.04$. Group (1) with the larger $m$ was also better correlated with the data than group (2) suggesting that commuters coming from the combination of land-use entities located along the MRT line in group (1) have mobility pattern that is more predictable.

To investigate the source of discrepancies between our model and the data, we looked at the standard deviations provided by the modified radiation model, $\sigma_{\text {model }}$, and those derived from the data, $\sigma_{d a t a}$. The modified radiation model gives a multinomial distribution of travels, hence variance of the predicted travel demand values 


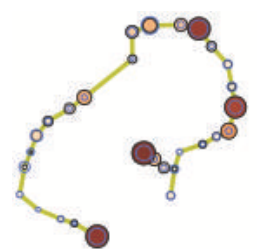

0.77

(a) Circle line

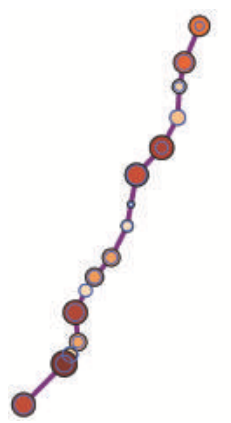

0.69

(c) North East line

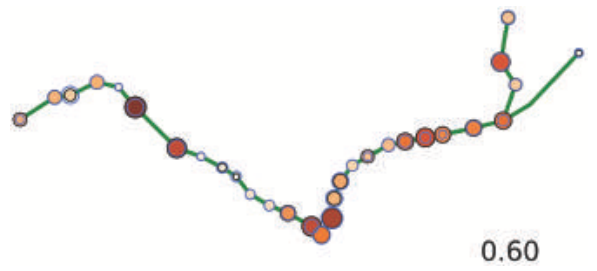

(b) East West line

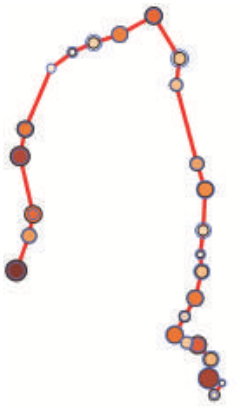

0.57

(d) North South line

Figure 5.10: Travel demand prediction for MRT lines. Correlation of predicted values with data for the (a) Circle, (b) East West, (c) North East and (d) North South lines.

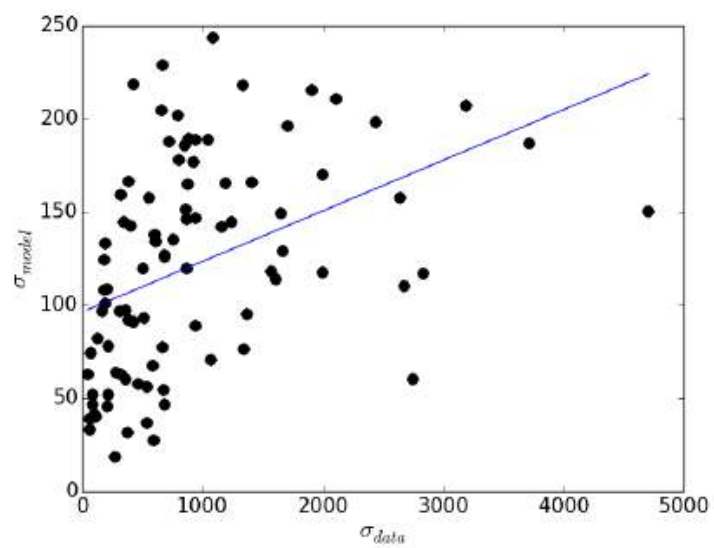

(a)

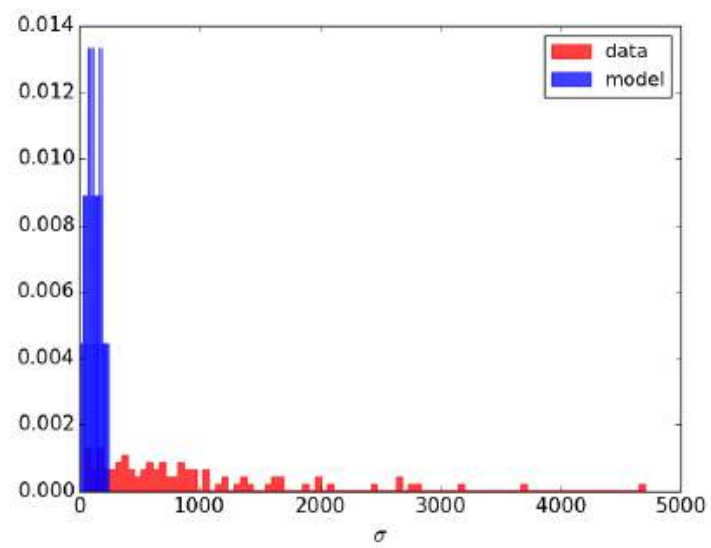

(b)

Figure 5.11: Standard deviations of station travel demand. The travel demand standard deviations of the model and the data are shown in (a) with the blue line being the best fit line. (b) shows the probability distributions of the standard deviations of the model and data. The data has higher variances than model predictions. 


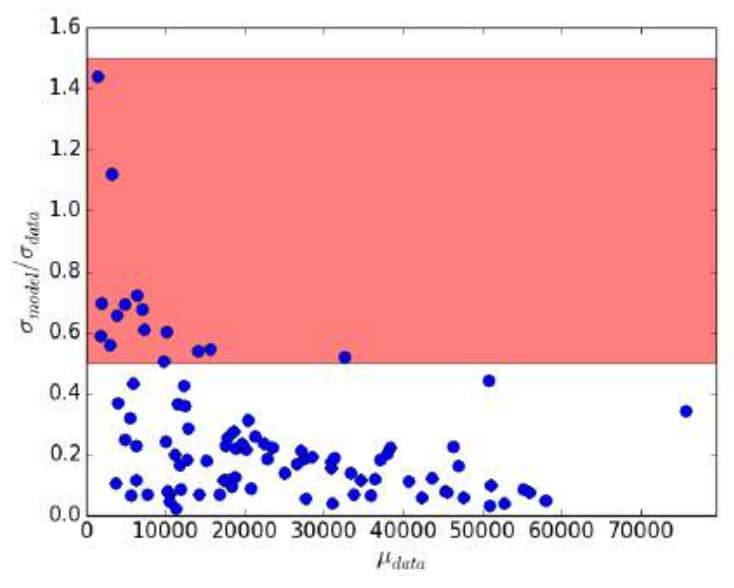

(a)

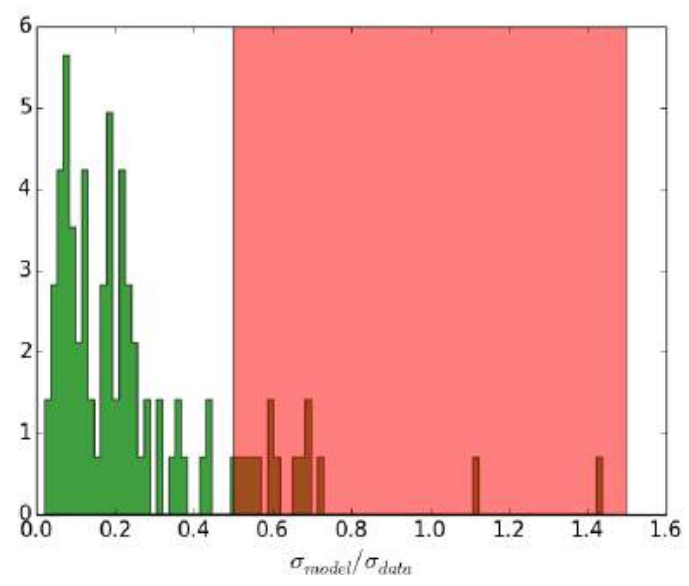

(b)

Figure 5.12: Proportion of actual travel demand fluctuations captured by model. The proportion of the standard deviations of the model and the data, $\sigma_{\text {model }} / \sigma_{\text {data }}$, for the mean data travel demand $\mu_{\text {data }}$ is shown in (a). (b) shows the histogram of $\sigma_{\text {model }} / \sigma_{\text {data }}$. The stations within the red region represent those for which $\sigma_{\text {model }}$ is within $50 \%$ of $\sigma_{\text {data }}$.

as given by our model is

$$
\sigma_{\text {model }}^{2}=\left(g_{i}^{O} N_{c}\right) p_{i j}\left(1-p_{i j}\right)
$$

where

$$
p_{i j}=\frac{g_{i}^{O} g_{j}^{D}}{\left(g_{i}^{O}+s_{i j}^{D}\right)\left(g_{i}^{O}+g_{j}^{D}+s_{i j}^{D}\right)} .
$$

Comparing the standard deviations of the model and the data in Fig. 5.11, we see that the data has a wider range of standard deviations than the model and that linear relationship between them can only be possible for $\sigma_{\text {data }}<2000$. The modified radiation model is unable to capture large variations in travel demand.

$\sigma_{\text {model }}$ being dependent on GPR values which are effectively measures of population density may be performing poorly in areas of high populations. Looking at the proportion of $\sigma_{\text {data }}$ captured by $\sigma_{\text {model }}$ as in Fig. 5.12, we find that the model is able to estimate fluctuations in the travel demand of stations up to half of the actual standard deviations when usage of the station (i.e. population around the station) is low (less than 15,000 commuters per day). But this is true for only a small percentage of the stations. In addition, we see that the model can perform 


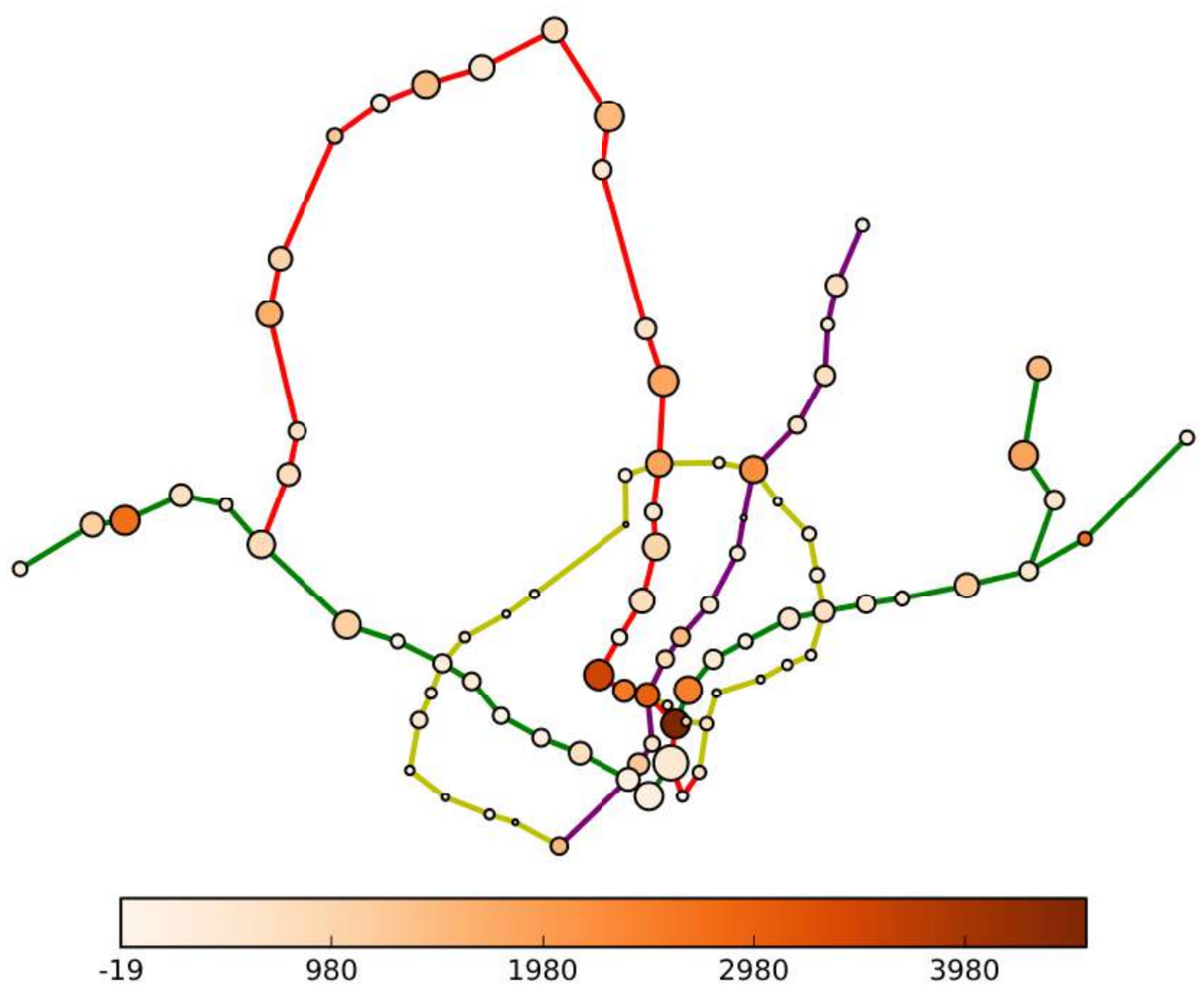

Figure 5.13: Difference in fluctuations of model and data. The size of the nodes corresponds to $\mu_{\text {data }}$. Nodes are colored according to $\sigma_{\text {data }}-\sigma_{\text {model }}$.

poorly regardless of the actual station travel demand suggesting that there exists another crucial factor as to why fluctuations are not reproduced.

From Fig. 5.13, we find that the errors in fluctuations are concentrated in the central business district area characterized by the highest frequency of commuters moving at the fastest time scales around stations that are highly clustered in space. This hints at the possible limitations of the model. It suggests the inability of the model to capture the fluctuations in travel demand in regions of small area, high density, and high activity, as we notice the radiation framework underestimating the behavioral changes in movement of the commuters. This inadequacy of the model is deemed to result from the spatial resolution in these areas of highly clustered stations to be far too fine-grained. Another possible reason for the insufficiency of the model is its neglect of a multitude of additional motivations driving a large proportion of commuter movements in the business and commercial centers beyond 
simply just home-to-work and work-to-home journeys.

\subsection{Summary and Conclusions}

Interactions and movements in urban systems are controlled by their transportation networks. A public transportation system capable of moving individuals at higher volumes and frequency but with less resources is an essential component that drives the economic progress of a city. For these systems to function efficiently in the midst of a continual growth in population size, there is a need to build a robust network. Among these systems, the Rapid Transit System (RTS) is becoming the most utilized mode of public transportation because of its high capacity, high frequency and grade separation from other modes of transportation [6]. The increasing use of RTS makes it a key indication of urbanization.

In this chapter, we have enhanced the radiation model by coupling land-use and the network structure of RTS into its modeling paradigm to predict urban travel demands. We have applied the model to the Singapore Mass Rapid Transit (MRT) System. We found that considering the node degree centrality of the network and using the gross plot ratio with entropy measure as the potential indicator of how likely a commuter will be attracted to travel to specific areas, the predicted values of the model has a correlation of 0.70 with data. Considering morning and afternoon trips gives a slightly better correlation of 0.74 and 0.71 respectively. Our results here also outperform those determined from the gravity model and the original radiation model. Although not provided here, with this model, one can perform predictions on travel time delay when land-use pattern is changed and when network structure is changed. It can also be used to investigate if there is an optimal network structure for the existing land-use pattern. Similarly, it can also be employed to determine how land-use should be designed for an existing transport network. Scenarios involving land-use design and transport structure and demand can be explored with this model.

In summary, we have enhanced the original radiation model through increasing its resolution in depicting the factors that drive the commuters to travel in each region. These factors are land-use, transport network, and amenities. By considering these microscopic features, the modified radiation model is now able to account for 
spatial heterogeneity which is deficient in the original model. In fact, it can also provide insights as to how these features co-evolve with each other. For example, while a network of public transport system determines how the residents of an urban city move, the transport network is also shaped by the changing human mobility and land-use patterns. For a transport network to be efficient and robust, its structure should evolve together with the changing land-use patterns of its associated urban area. In this chapter, we have explored the effects of land-use, transport network, and amenities on the spatio-temporal mobility of commuters in the Singapore MRT system. Although we have presented our application of the modified radiation model to the case of Singapore MRT system, we expect it to be easily extensible to account for the travel demands in other cities of the world.

\subsection{Future Work}

We have presented here how our modified radiation model (MRM) can be applied to intra-city travels in the context of the Singapore RTS. To further investigate the generability of our framework to intra-city travels, a future direction can be testing it in other cities and with other modes of transportation. Additionally, most urban dwellers commute by using a mix of transport modes. In this sense, an extension of the framework for a network of transport networks is suggested. When commuters use both RTS and bus in one journey then we can model the movement in terms of a network of RTS and bus system.

In estimating the density of opportunities, a finer scale of granularity can be tested. What if an establishment-level of amenities information is used instead of a land-use plan? Such an establishment-level information can be procured from publicly available maps like the OpenStreet Maps. Other ways of quantifying "opportunities" and their density can also be explored.

Although not provided here, with this model, one can perform predictions on travel time delay when land use pattern is changed and when network structure is changed. It can also be used to investigate if there is an optimal network structure for the existing land use pattern. Similarly, it can also be employed to determine how land use should be designed for an existing transport network. Scenarios involving land use design and transport structure and demand are suggested to be explored 
with this model.

Finally, the model in its current form gives the probability distribution of travels in space but not in time. Further works can concentrate on how a dynamic radiation model based on first principles can be derived. In such a case we will have probability trip distributions in both space and time. 


\section{Chapter 6}

\section{Fragmentation and Resilience of Networks}

The dynamics of how individuals navigate space is influenced by the available infrastructure and the existing socio-economic ties. The frequency distribution of regional and local travels is affected by the accessibility of each area as determined by the structure of the transport network - which we explored in the previous chapter and was also investigated in other works in the context of different cities and spatial scales $[73,74,90,91,76,92,80,93,94,95]$. Additionally, the attractiveness of travelling to a certain area is shaped by the extent and strength of social and economic connections [96, 97, 98, 99].

As the network structures present in the system affect mobility, the interactions of individuals in return may also cause these networks to change. To capture how systems change, it is necessary to understand how agents are connected and how links are formed and broken. In this chapter, we concentrate on the factors that drive the breakage of links in a network. Here, we move beyond transport networks with the aim of investigating the effect of interactions (i.e. of any kind, not just mobility) on the resilience, integrity and evolution of spatial networks. We present a general framework for probing the dynamic evolution of spatial networks by considering the forces that nodes apply along their links. We show that not only the underlying structure but also the magnitude and direction of forces on the network affects the ability of a sytem to withstand perturbations, may these be random failures, deliberate attacks or accumulation of small but continuous forcings.

Upon reaching a critical amount of accumulated force, there are systems which 
experience sudden collapse. Road network links fail when vehicular congestion disrupts the flow of traffic $[100,101]$. Protein chains are driven far apart when large stresses are present between connected molecules [102]. But there are also some networks which exhibit a high degree of resilience to forcings. Communication networks can continue to transmit information even during partial router malfunctions [103]. Organisms reproduce and propagate even after efforts are made to halt their growth. Their unexpected tolerance to breaking forces has been attributed to their underlying network structure $[1,104]$. We further show here, that the magnitude and direction of interactions are equally important in determining a system's survival, and that small bursty breaking dynamics in the micro-scale can eventually lead to a macro-scale fragmentation.

In section 6.1, we discuss existing models that probe into the resilience of networks and how they can be measured when the interactions on them and/or the composition of their agents change. We then proceed to presenting our framework of network fragmentation. After discussing the insights we gained from the results of our model, we will illustrate how to apply this framework to real-world networks by using the mobility model presented in Chapter 5 to explore how the operations of a transport network might be disrupted as a result of sustained commuter flow. With our mobility model that estimates the throughput of a transport network given the land-use pattern in a region, we can compare relative lifetimes of different types of transport networks by incorporating Chapter 5's travel demand estimates into the dynamical model of network evolution presented in this chapter.

\subsection{Network resilience}

Resilience of a network is defined as the extent to which a network remains functional after node or link failures. It is characteristically measured by (1) the critical threshold, (2) size of the giant cluster, and (3) average cluster size [105]. Existing network resilience models that use the above measures can be categorized depending on the particular network fragmentation parameter they seek to study. In this section, we will discuss models focusing on three main parameters: (1) network structure, (2) network dynamics, and (3) failure mechanism [105]. 


\subsubsection{Measures of network resilience}

Failure of a single node may not compromise the integrity of a network. But successive and repeated failure of nodes can lead to the breakdown of the entire network. But how many nodes does it take for a network to collapse? In the case of telecommunications, how many cell towers have to malfunction for the entire network to become a group of isolated components in which communication is impossible? We turn to percolation theory to offer us a mathematical foundation through which we can define and measure network resilience [106].

Percolation theory indicates the existence of a critical probability, $p_{c}$, below which the network is composed of isolated clusters. At $p_{c}$ a giant cluster of connected nodes spanning the entire network appears until it grows to a fully connected network (Fig. 6.1A) [107]. To describe this process, we use the following measures:

1. percolation threshold, $p_{c}$ - the critical probability at which small clusters grow and coalesce leading to the emergence of a giant connected component (Fig. 6.1),

2. size of giant cluster, $S$ - the number of nodes in the percolating cluster spanning the whole network (Fig. 6.1B), and

3. average cluster size, $\langle s\rangle-$ below $p_{c},\langle s\rangle$ is the average size of all clusters, and above $p_{c},<s>$ is the average size of all clusters except for the giant cluster (Fig. 6.1C).

Network fragmentation is essentially an inverse percolation process. In this case, the removal of $f$ fraction of nodes can impact the integrity of the network $[106,107]$. For small $f$, the failure of some nodes does little damage to the network. Increasing $f$ leads to a decrease in the size of the giant cluster of connected components, until a suffiently large $f$ wherein the giant cluster breaks into many small isolated clusters $[106,107]$. Similar to percolation, this gradual fragmentation process can be characterized by:

1. critical fragmentation threshold, $f_{c}$ - minimal fraction of nodes or links whose failures lead to network collapse or a sudden transition to a network of many isolated components, 
a

$$
\mathrm{p}<\mathrm{p}_{\mathrm{c}}
$$

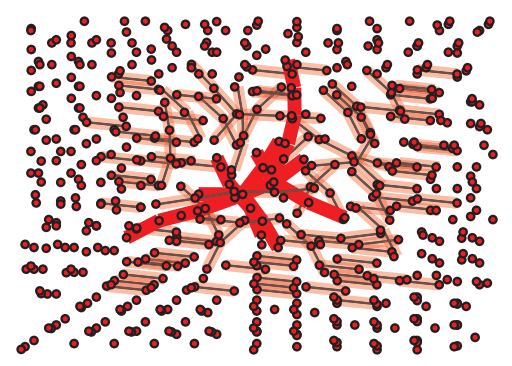

$$
p \sim p_{c}
$$

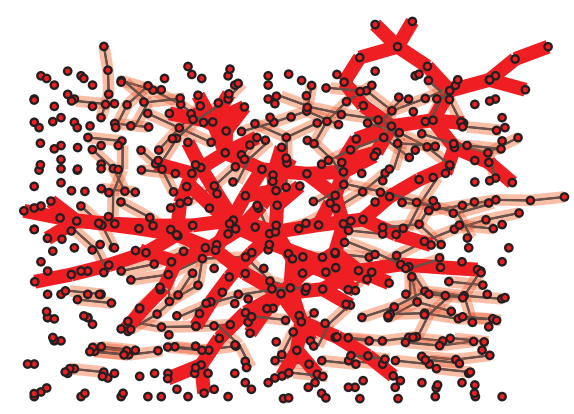

b

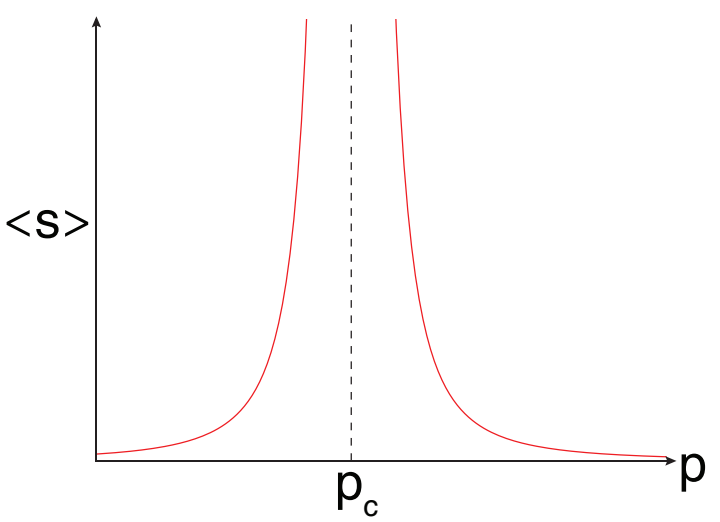

$p \sim p_{c}$

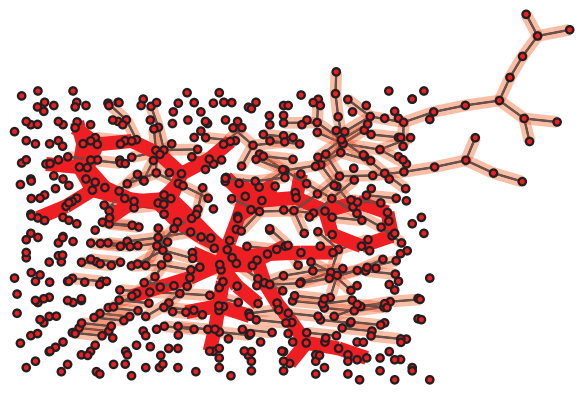

$p>p_{c}$

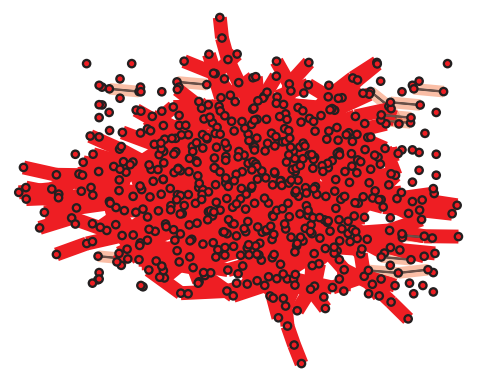

C

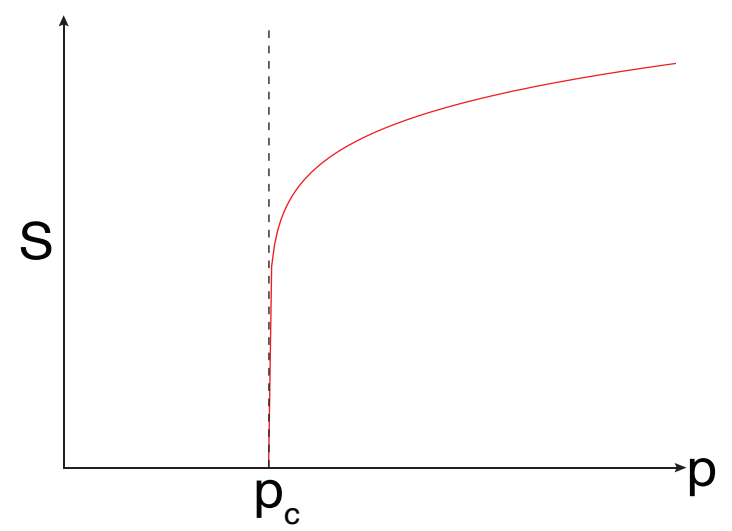

Figure 6.1: Percolation. (a) Below the critical probability, $p_{c}$, the network is composed of isolated clusters. Above $p_{c}$, a giant cluster spans the network. (b) The average cluster size, $\langle s\rangle$ diverges at $p_{c}$. (c) A giant cluster appears at $p_{c}$ and grows until the network is fully connected. 
2. size of giant cluster, $S$ - maximum number of nodes connected in the network during every stage of its evolution,

3. average cluster size, $\langle s\rangle-$ below $f_{c},\langle s\rangle$ is the average size of all clusters except for the giant cluster, and above $f_{c},\langle s\rangle$ is the average size of all clusters.

\subsubsection{Models of network resilience}

We can categorize studies of network resilience according to which parameter affecting the robustness of a network they focus on.

\subsubsection{Network structure}

The resilience of a system is deeply influenced by the structure of its network. The distribution of force and the global impact of node failures are dependent on how nodes are connected in the network. Considering a static disturbance, several models have been developed to analyze the resilience of networks to one time deletion of edges including random failures and targeted removals [1, 104, 108, 109]. All of them indicate that there is a strong correlation between network resilience and structure $[1,104]$.

In a study comparing the robustness of an Erdos-Renyi random network with a Barabasi-Albert scale-free network, it was found that scale-free networks are more robust than random networks against random node failures (blue symbols in Fig. 6.2), but are more vulnerable to attacks (red symbols in Fig. 6.2) targetting the most highly connected nodes $[1,104,107]$. The heterogeneous topology of scale-free networks in which low-degree nodes are far more abundant than high-degree nodes results to the tolerance of scale-free networks to random errors [1, 104, 107, 108, 109]. It is more probable in a scale-free than a random network, to select a node which only has a marginal effect in the overall integrity of the network [107]. This same heterogeneity in degree distribution, causes scale-free networks to be extremely prone to collapse as the intentional attack on their most connected node results to a massive disruption in their function as their highest degree node has far more connections than that of random networks $[1,104,107,108,109]$. 

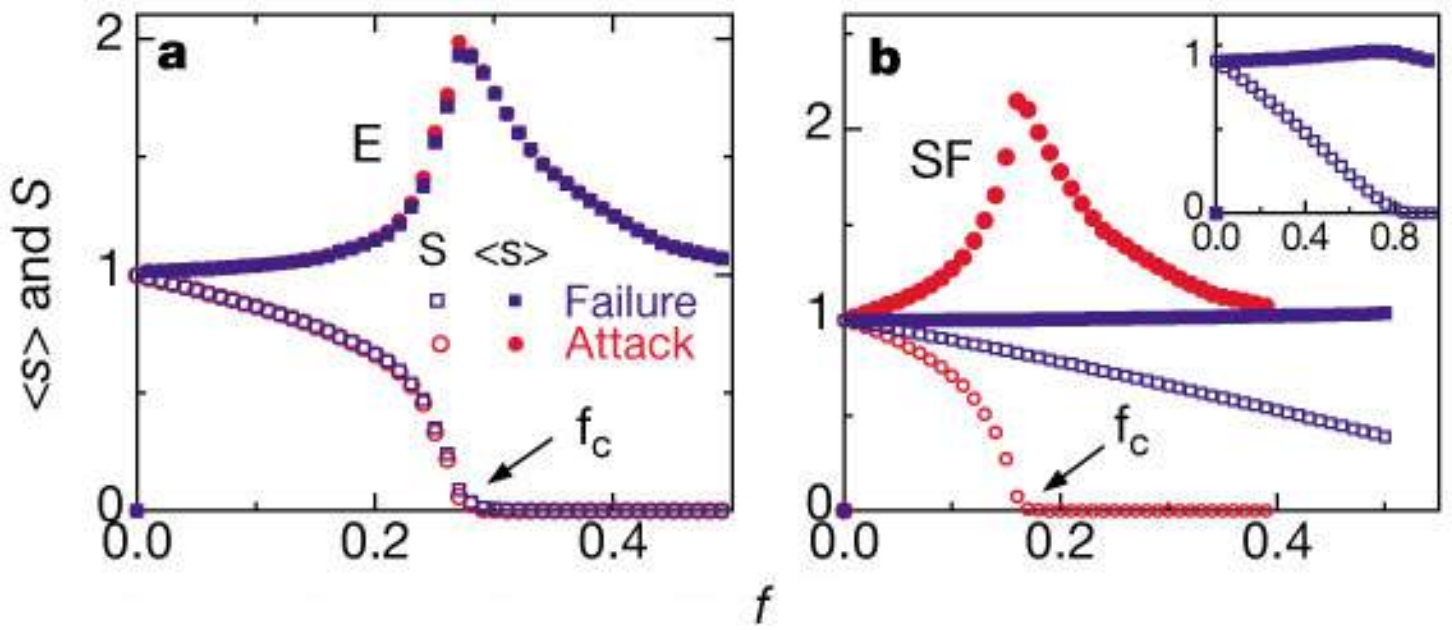

Figure 6.2: Error and attack tolerance. The relative size of the giant cluster $S$ (open symbols) and the average size of isolated clusters $\langle s>$ (filled symbols) as a function of the fraction of removed nodes $f$ are shown. (a) Fragmentation of the exponential random network, E, under random failures (squares) and attacks (circles). (b) Fragmentation of the scale-free network, SF, under random failures (blue squares) and attacks (red circles). The inset in (b) shows the error tolerance curves for the whole range of $f$. Scale-free networks are more robust than random networks against random node failures but are more vulnerable against targeted attacks. (Figure from [1].) 


\subsubsection{Network dynamics}

The networks considered above are assumed to be static. However, many real networks are dynamically evolving [105, 107]. Traffic flows in transport networks differ depending on the time of the day [110]. Interactions in a gene regulatory network vary depending on the environmental conditions [111]. Network resilience in such cases are time-dependent. The extent of the effect of a node removal is determined not only by network structure but also when the removal happens.

Furthermore, dynamical resilience of networks differ from static resilience, in that the former refers to the ability of the network to maintain its dynamical functions even under perturbations [112]. In contrast to static structural fragility, the dynamics of a network with a heterogeneous node degree, such as in a scale-free network, can be highly vulnerable even to the failure of low-degree nodes [112]. This crucial role of low-degree nodes results from the dynamical process involving a reduction in the activity of normal nodes whenever they compensate for the failure of their neighboring nodes [112].

\subsubsection{Failure mechanism}

The removal of a node can also affect the functionality of other nodes leading to a cascade of failures affecting a large fraction of the network [107]. Consider the Internet as an example. The removal of a highly connected router will redirect traffic to other routers which may not have the capacity to handle a large amount of additional traffic causing them to issue a denial of service [107]. Watts has shown that the structure of the network also affects its robustness under such cascade of failures [113]. It was found in his model that a more heterogeneous node degree distribution reduces the likelihood of a global cascade of failures [113].

For some real-world networks, a significant amount of their damaged parts is capable of regaining functionality causing the system to spontaneously recover after breakdown [105]. The human brain has the ability to recover after an epileptic attack [114]. Small vehicular adjustments can relax traffic gridlock. A financial market may bounce back and recover after a period of inactivity. A framework for understanding the ability of such dynamical networks to recover after collapse was developed by Majdandzic et al. [115]. Using a stochastoc contiguous spreading model, they found that local node recoveries can lead to the spontaneous emergence 
of macroscopic network recovery [115].

\subsubsection{Spring-mass model of network fragmentation}

Although models of dynamic network fragmentation have been developed, these models fail to capture the gradual but continuous breaking that is always at play in many networks. A network can initially demonstrate resilience but when continuously subjected to force, the accumulated damage can lead to network fragmentation or collapse. The crucial parameter we have in our model that is lacking in previous models is the inclusion of deformation memory. The damage sustained by a network at a particular time affects the resilience of the network not only at that time but also at succeeding times. The distribution of forces in the network constantly changes every time an edge breaks and depending on how the edges are directed and how the nodes are connected, the final topology of the network may vary.

Here, we analyze the resilience to edge failures of lattice networks with only shortrange links, and those with both short and long-range links such as Erdos-Renyi (ER) and Barabasi-Albert (BA) networks by following their evolution as they continuously experience a constant amount of force among their nodes. We consider network types that are distributed in a spatial grid as widely seen in distributed parallel computing [103], router and sensor networks $[103,109,116]$, distributed control manufacturing systems [103], satellite constellations [103], wired circuits [103], transport systems $[117,100,101]$, electrical power grids $[117,109,116,118]$ and protein chain networks [102]. We also present how any network spatial configuration can be embedded into a spatial grid and demonstrate this with an actual bus transport network.

Some of the factors that influence the disconnection of links in evolving networks are force magnitudes and duration of time these forces are applied. Here, we add to existing works on network resilience by showing that the robustness and tolerance of a spatial grid network to edge failures is also contingent crucially on the direction of edges along which forces in the system are applied. Choosing the appropriate connection and direction patterns of the edges can optimize the distribution of load in the system and can consequently make a network more resilient to edge failures [109]. We consider two different edge direction patterns for the lattice, ER, and BA networks, (A) uniform force directions and (B) random force directions. 


\subsection{Spatial grid network}

Much of the work concerning network modelling do not incorporate the spatial positions of the nodes in the system. But as argued by Gastner et al., nodes of realworld networks have well-defined positions that change over time, and incorporating spatial and temporal information into the analysis of networks give a better insight as to how a system behaves [100]. We consider lattice, ER, and BA networks embedded in Euclidean grid space [100] where the edges can either represent physical constructs or some abstract relationship.

Nodes with identical masses $m$ are positioned in an $M \times N$ lattice labelled by $(i, j)$ where $1 \leqslant i \leqslant M$ and $1 \leqslant j \leqslant N$ as illustrated in Fig. 6.3. Taking into consideration the ease of computation, the motion of each $(i, j)^{\text {th }}$ node is constrained to two dimensions $x_{i, j}$ and $y_{i, j}$. Each edge is an elastic simple Hookean spring with stiffness $\kappa$ and unstretched length $l_{0}$. By virtue of being connected, two nodes experience a Hookean spring force proportional to the elongation of their edge $\Delta l \equiv$ $l-l_{0}$ with $\kappa$ being the constant of proportionality.

The nodes in the spatial grid can either be connected following the configuration of a lattice network (Fig. 6.3), an ER network (Fig. 6.4), or a BA network (Fig. 6.5). In the lattice network, connections are made using the Von Neumann neighborhood, forming $[(M-1) \times N]+[M \times(N-1)]$ connections. Moreover, free boundary conditions (FBC) are imposed. To keep the number of connections comparable across all networks considered, an approximately equivalent number of edges is formed for the ER and BA networks.

The directions of the edges represent the force directions in the grid network. The force directions in the network can either follow the uniform connection pattern shown in Fig. 6.3(a) and Fig. 6.4(a) or the random connection pattern shown in Fig. 6.3(b) and Fig. 6.4(b). For BA networks with uniform force directions, almost all edges were directed outward and all non-zero node in-degrees are only $k_{i n}=2$. On the other hand, for BA networks with random force directions, the number of edges directed outward is about the same as those directed inward as shown by their similar node in-degree and out-degree distributions in Fig. 6.5. 

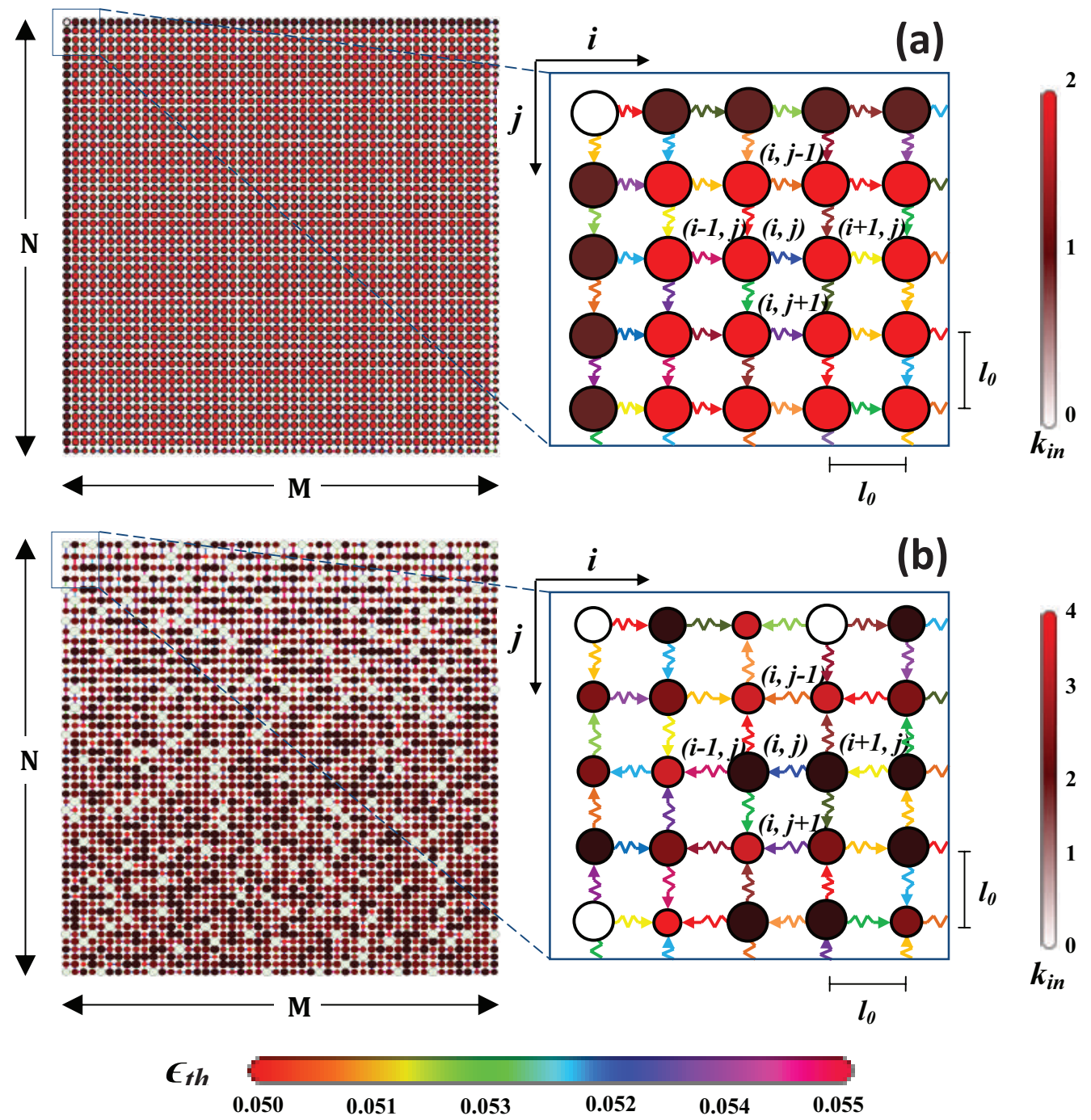

Figure 6.3: Lattice network architecture and index conventions. (a) represents a lattice network with uniform force directions and (b) random force directions. Edge colors correspond to the elongation thresholds $\epsilon_{t h}$ while node colors are based on the degree of incoming connections $k_{i n}$. Node sizes correspond to the degree of outgoing connections $k_{\text {out }}$ (largest node has higher $k_{\text {out }}$ ). Arrows are directed from source to target node. 

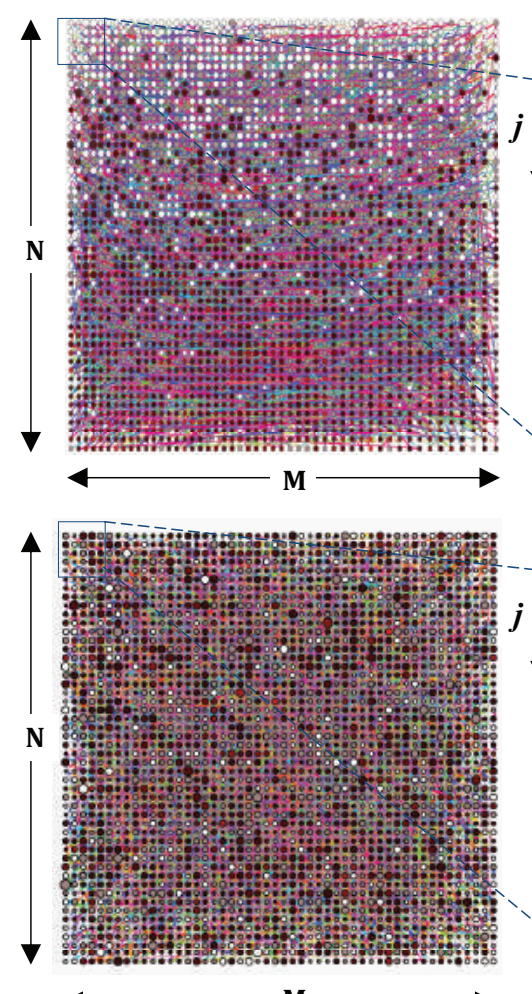

$\mathbf{M}$
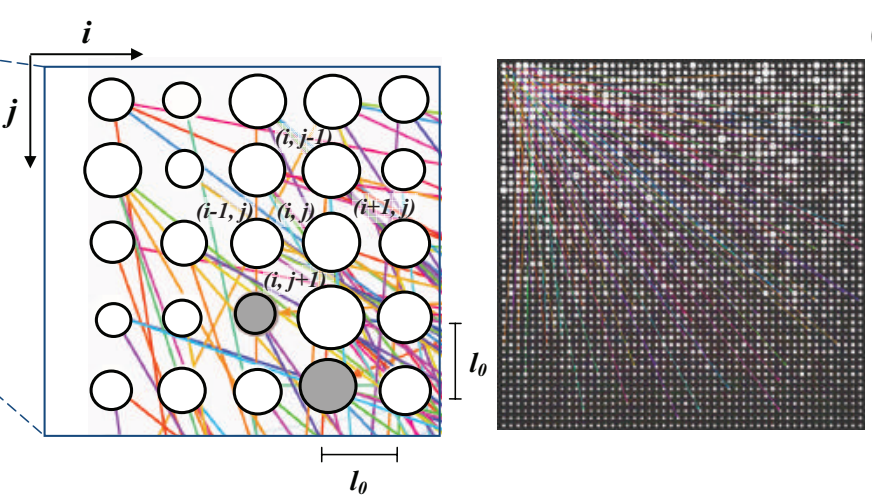

(a)

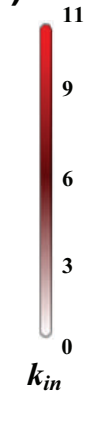

(b)
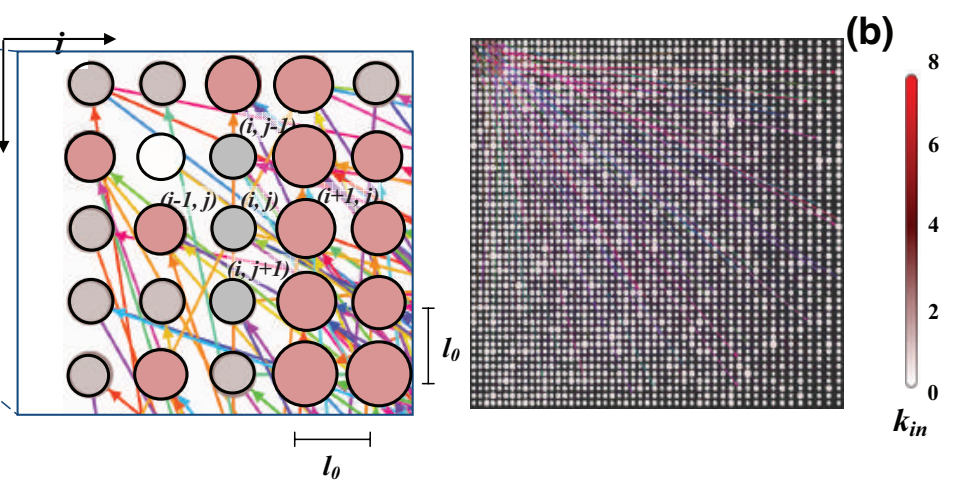

\section{.}

$\boldsymbol{\epsilon}_{\text {th }}$

Figure 6.4: Erdos-Renyi network architecture and index conventions. In (a) and (b) nodes are arranged spatially in a grid and $[(M-1) \mathrm{x} N]+[M \mathrm{x}(N-1)]$ edges are randomly chosen from possible connections. (a) represents an ER network where forces are directed with the highest level of uniformity and (b) randomly. Edge colors correspond to the elongation thresholds $\epsilon_{t h}$ while node colors are based on the degree of incoming connections $k_{i n}$. Node sizes correspond to the degree of outgoing connections $k_{\text {out }}$ (largest node has higher $k_{\text {out }}$ ). Arrows are directed from source to target node. Figures in the last column highlight the edges of the shown selected nodes. 


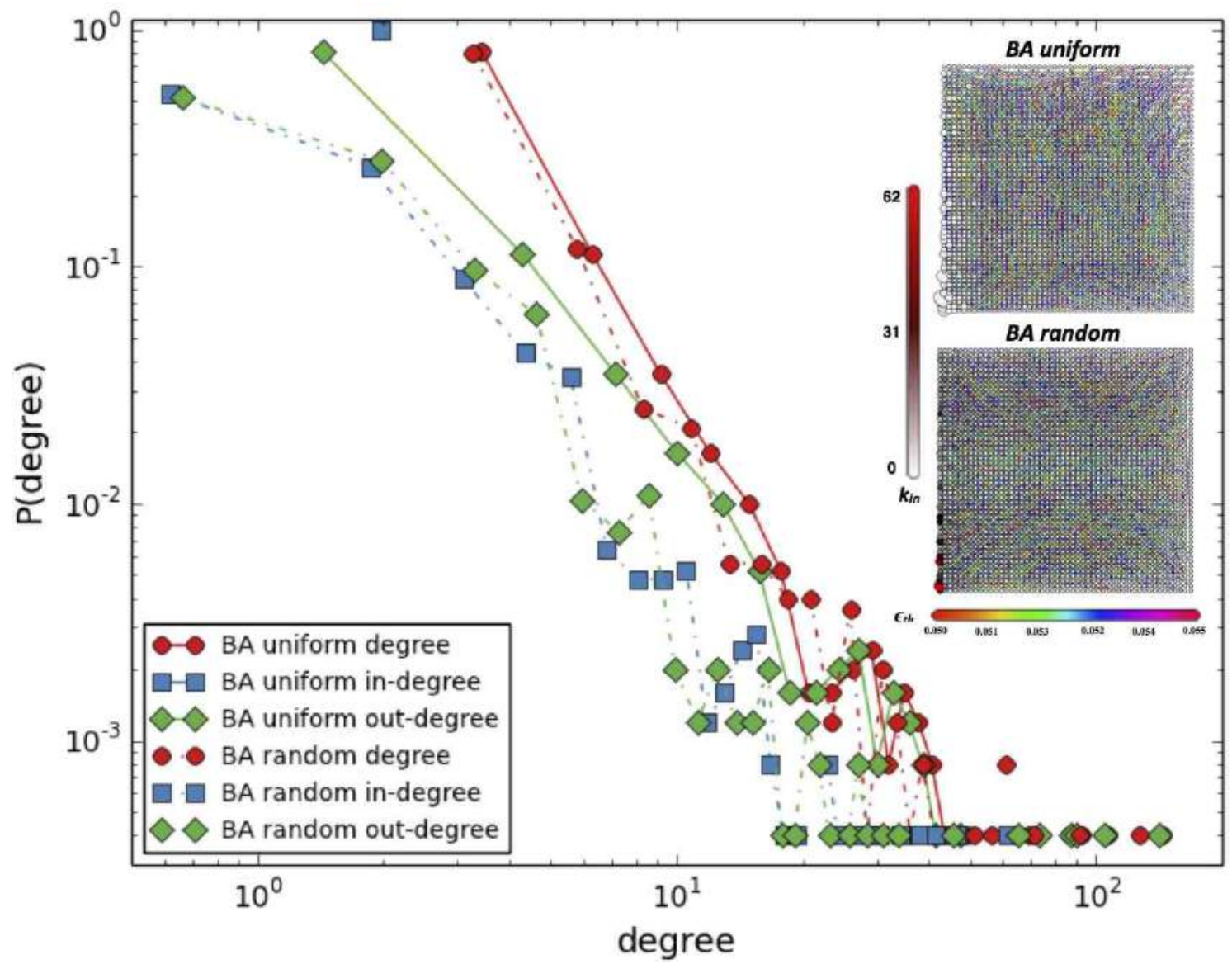

Figure 6.5: Barabasi-Albert network architecture and degree distribution with uniform and random force directions. Almost all edges were directed outward for the uniform case while comparable number of edges were directed inward and outward for the random case. In the inset, nodes are sized according to $k_{\text {out }}$. 


\subsubsection{Force accumulation}

Repeated application of force by the source or influential node to the target node, however small it may be, can eventually cause the failure of the edge linking them. The lengths of edges change at each time step as a result of such forcings. We denote this change in edge length as deformation $\varepsilon$. Following the work of Carmona et al. [119], each edge is given a memory of its deformation history $\{\varepsilon(0), \varepsilon(\delta t), \varepsilon(2 \delta t), \ldots, \varepsilon(t)\}$. At each time step, the elongation of the edge is computed and appended to the deformation history sequence. The edges have an infinite memory range which means that the entire deformation history is stored.

In determining whether a spring has broken or an edge has failed, we evaluate the total damage $q(t)$ of the spring as given by

$$
q(t)=\varepsilon(t)+f_{0} \sum_{t^{\prime}=0}^{t^{\prime}=t} \varepsilon\left(t^{\prime}\right),
$$

where $\varepsilon(t)$ is the instantaneous deformation and $f_{0} \sum_{t^{\prime}=0}^{t^{\prime}=t} \varepsilon\left(t^{\prime}\right)$ is the accumulated damage due to repeated application of force $[120,121]$. An edge fails and the connection is broken when the total damage $q(t)$ of a specific edge exceeds the elongation threshold $\varepsilon_{t h}$ of that edge. In the absence of memory, $\varepsilon(t)$ dominates and a network only fragments for force magnitudes above the limit. Accounting for memory allows subcritical loading where cyclical application of force causes progressive edge failures leading to a substantial fraction of edges to break. The contribution of the immediate deformation and the memory of accumulated damage is controlled by the parameter $f_{0}$ which we choose to be 0.05 for this study. At this value of $f_{0}$, the disorder in the fragmentation process is controlled and the effects of the interfering elastic waves are eliminated [121]. Choosing a smaller $f_{0}$ only lengthens all fragmentation processes and does not change any of our results.

\subsubsection{Breaking dynamics}

At the onset of the simulation, all nodes are at rest and located at their initial positions. The time evolution of the grid network is followed by solving the equations of motion of each of the nodes using an iterative fourth order Runge-Kutta method. As shown in the work of Esleta et al., a time step of $\delta t=2^{-10}$ produces a total 
accumulated global error of $\delta \xi \sim 9 \times 10^{-4}$ which causes no significant position and velocity errors [120]. The equations of motion is a set of second-order coupled differential equations given by

$$
m \ddot{\vec{r}}_{(i, j)}=\vec{F}_{(i, j)}
$$

where $r_{(i, j)} \equiv x_{(i, j)} \hat{e}_{x}+y_{(i, j)} \hat{e}_{y}$ and $\Delta \vec{r}_{(i, j)}(t) \equiv\left[x_{(i, j)}(t)-x_{(i, j)}(t-1)\right] \hat{e}_{x}+\left[y_{(i, j)}(t)-\right.$ $\left.y_{(i, j)}(t-1)\right] \hat{e}_{y}$ is the displacement of mass $(i, j)$ from its initial position in the grid [120]. The total force $\vec{F}_{(i, j)} \equiv F_{(i, j)}^{x} \hat{e}_{x}+F_{(i, j)}^{y} \hat{e}_{y}$ acting on mass $(i, j)$ is a combination of the force applied along network links, $\vec{F}_{\text {app }}$, and the interpaticle forces, $\vec{f}$, resulting from spatial nearest neighbor interactions

$$
\vec{F}_{(i, j)} \equiv \vec{F}_{a p p}+\vec{f}_{(i, j+1)-(i, j)}+\vec{f}_{(i-1, j)-(i, j)}+\vec{f}_{(i+1, j)-(i, j)}+\vec{f}_{(i, j-1)-(i, j)}
$$

Force is applied between connected nodes and has the same magnitude for all edges. The directions of the applied forces $\left\{F_{a p p}^{k}\right\}$ were either (A) uniform or (B) random. In method $\mathrm{A}$, the directions of $F_{a p p}$ were made to have the highest degree of uniformity (Supplementary Fig. A.2, Fig. A.4 and Fig. A.6). In method B, we scan all edges of the grid network at the start of the simulation, and for each edge, we choose a node randomly and assign this node as the target node (Supplementary Fig. A.3, Fig. A.5 and Fig. A.7). The applied force is then directed from source to target node. Doing so, allows us to explore whether the final fragmented structure of the grid network is affected by the direction of $F_{a p p}$. Each network simulation was run for at least three realizations.

The displacement of each node of mass $m$ is then computed according to the breaking dynamics of the configuration in Fig. 6.6. If the displacement of a particular node causes one of its edges to elongate beyond its threshold then the link is considered broken and removed from succeeding iterations of the simulation.

The interparticle forces acting on node $(i, j)$ is different from $\vec{F}_{a p p}$ in that it can exist even if there is no link connecting one node to another as long as the nodes are nearest neighbors in the spatial lattice. It is solved by computing for the deformation vector $\vec{\varepsilon}_{(i \pm 1, j \pm 1)-(i, j)}$ of its edges [120]. From the geometry of the system as shown 


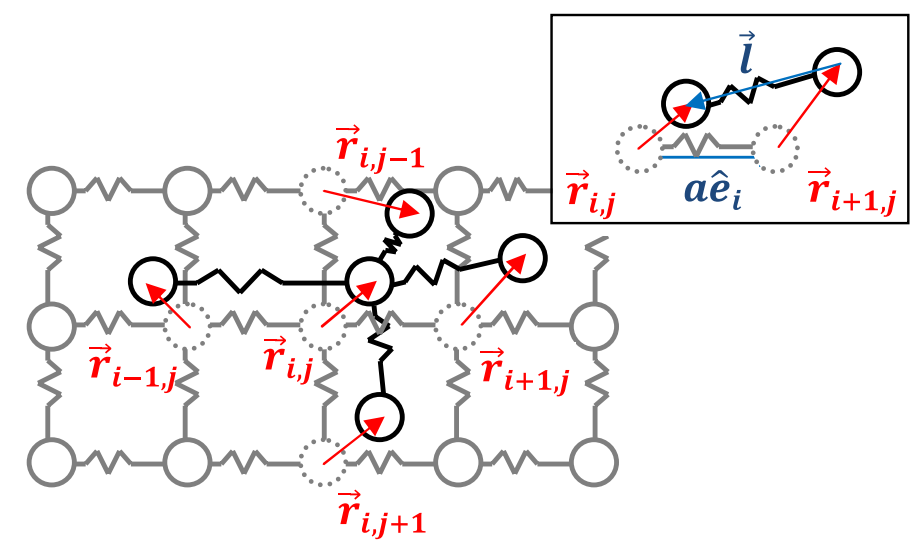

Figure 6.6: Body diagram. Displacement of the $(i, j)^{\text {th }}$ node and its four nearest neighbors.

in Fig. 6.6, the horizontal deformations are

$$
\begin{aligned}
& \varepsilon_{(i, j+1)-(i, j)}^{x}=\left[1-\frac{l_{0}}{\sqrt{\left(x_{i, j}-x_{i, j+1}\right)^{2}+\left(y_{i, j}-y_{i, j+1}-l_{0}\right)^{2}}}\right]\left(x_{i, j}-x_{i, j+1}\right) \\
& \varepsilon_{(i-1, j)-(i, j)}^{x}=\left[1-\frac{l_{0}}{\sqrt{\left(x_{i, j}-x_{i-1, j}+l_{0}\right)^{2}+\left(y_{i, j}-y_{i-1, j}\right)^{2}}}\right]\left(x_{i, j}-x_{i-1, j}+l_{0}\right) \\
& \varepsilon_{(i+1, j)-(i, j)}^{x}=\left[1-\frac{l_{0}}{\sqrt{\left(x_{i, j}-x_{i+1, j}-l_{0}\right)^{2}+\left(y_{i, j}-y_{i+1, j}\right)^{2}}}\right]\left(x_{i, j}-x_{i+1, j}-l_{0}\right) \\
& \varepsilon_{(i, j-1)-(i, j)}^{x}=\left[1-\frac{l_{0}}{\sqrt{\left(x_{i, j}-x_{i, j-1}\right)^{2}+\left(y_{i, j}-y_{i, j-1}+l_{0}\right)^{2}}}\right]\left(x_{i, j}-x_{i, j-1}\right),
\end{aligned}
$$

while the vertical deformations are

$$
\begin{aligned}
& \varepsilon_{(i, j+1)-(i, j)}^{y}=\left[1-\frac{l_{0}}{\sqrt{\left(x_{i, j}-x_{i, j+1}\right)^{2}+\left(y_{i, j}-y_{i, j+1}-l_{0}\right)^{2}}}\right]\left(y_{i, j}-y_{i, j+1}-l_{0}\right) \\
& \varepsilon_{(i-1, j)-(i, j)}^{y}=\left[1-\frac{l_{0}}{\sqrt{\left(x_{i, j}-x_{i-1, j}+l_{0}\right)^{2}+\left(y_{i, j}-y_{i-1, j}\right)^{2}}}\right]\left(y_{i, j}-y_{i-1, j}\right) \\
& \varepsilon_{(i, j-1)-(i, j)}^{y}=\left[1-\frac{l_{0}}{\sqrt{\left(x_{i, j}-x_{i, j-1}\right)^{2}+\left(y_{i, j}-y_{i, j-1}+l_{0}\right)^{2}}}\right]\left(y_{i, j}-y_{i, j-1}+l_{0}\right) \\
& \varepsilon_{(i+1, j)-(i, j)}^{y}=\left[1-\frac{l_{0}}{\sqrt{\left(x_{i, j}-x_{i+1, j}-l_{0}\right)^{2}+\left(y_{i, j}-y_{i+1, j}\right)^{2}}}\right]\left(y_{i, j}-y_{i+1, j}\right) .
\end{aligned}
$$

The edges are assumed to be Hookean springs and as such the interparticle force is $\vec{f}_{(i \pm 1, j \pm 1)-(i, j)}=-\kappa \vec{\varepsilon}_{(i \pm 1, j \pm 1)-(i, j)}$. Using the deformations given by equations 6.4 and 6.5 to derive the interparticle forces exerted by the springs and combining them 
with $\vec{F}_{a p p}$, the horizontal component of the total force $\vec{F}_{i, j}$ acting on $(i, j)$ is

$$
F_{i, j}^{x}=F_{a p p}^{x}+\kappa\left[\varepsilon_{(i, j+1)-(i, j)}^{x}-\varepsilon_{(i-1, j)-(i, j)}^{x}+\varepsilon_{(i+1, j)-(i, j)}^{x}-\varepsilon_{(i, j-1)-(i, j)}^{x}\right]
$$

while the vertical component is

$$
F_{i, j}^{y}=F_{a p p}^{y}+\kappa\left[\varepsilon_{(i, j+1)-(i, j)}^{y}-\varepsilon_{(i-1, j)-(i, j)}^{y}-\varepsilon_{(i, j-1)-(i, j)}^{y}+\varepsilon_{(i+1, j)-(i, j)}^{y}\right]
$$

After solving for the displacement of each node in the grid network, the condition for breaking is evaluated for all intact edges. Although the equation of motion considered does not include a velocity-dependent term, the networks studied still take this into account by considering the entire history of deformations which together with the instantaneous force can cause a link to break. If the elongation of the spring exceeds $\varepsilon_{t h}$, the edge is removed from further calculations. The elastic energy of the broken link is released and redistributed to the edges of its nearest connected nodes [120]. The breakage of a particular link causes cascading disconnections and may eventually lead to network collapse.

\subsection{Lattice network}

The spatial arrangement of nodes in a network affects the distribution of force in the system and dictates the degree of influence a node has as compared to other nodes. In some cases, direct interactions among components are only possible between spatial neighbors. We analyze this configuration using a 50 x 50 lattice network with 4,900 edges.

\subsubsection{Uniform force directions}

The forces in a system can be directed uniformly such that all vertical and horizontal $F_{a p p}$ have the same directions. This results to a net force directed diagonally in the network. Such forcing pattern also allows nodes to be influenced only by nodes that are to their right and below them (see Supplementary Section A.3.1.1).

As the network evolves, a cascading disconnection of edges is observed as shown in Fig. 6.7. The first to break are the edges near the top and left sides since they feel 

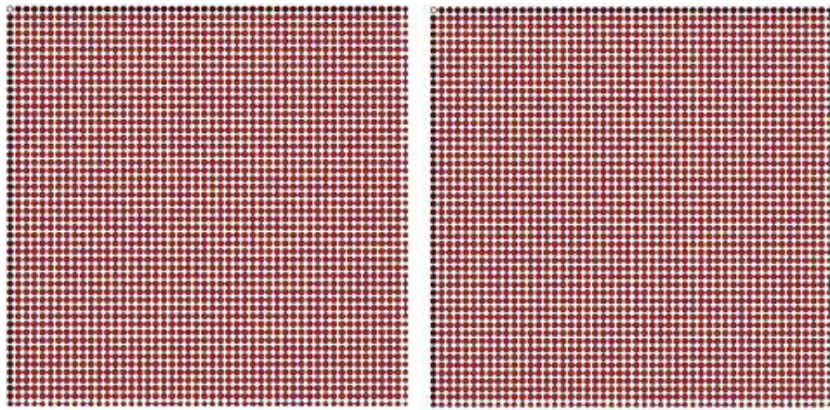

$\mathbf{t}=\mathbf{0 . 0}$

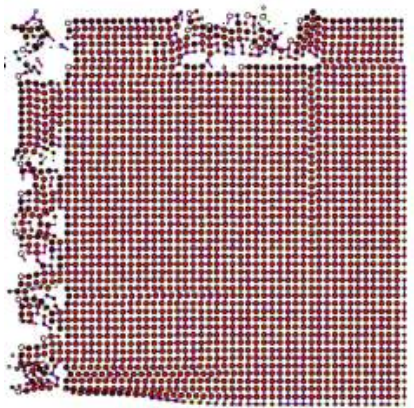

$t=6409.0$ $\mathbf{t}=\mathbf{3 0 0 0 . 0}$

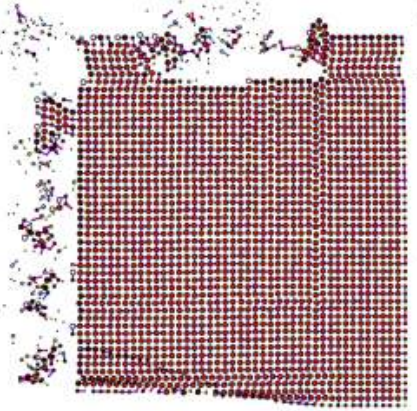

$t=6421.0$

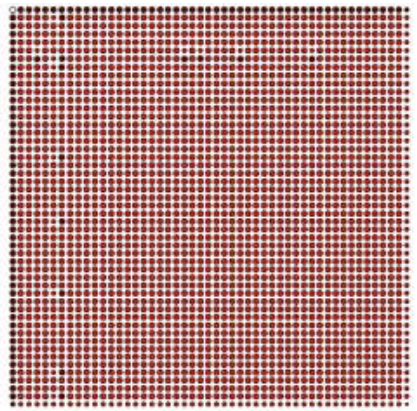

$t=6384.0$

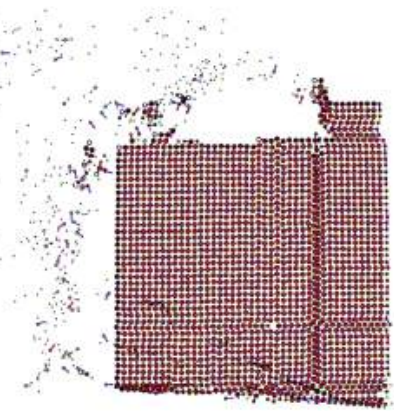

$t=6434.0$

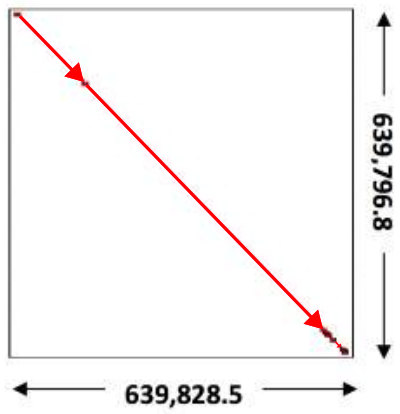

network movement

Figure 6.7: Evolution of a lattice network with uniform force directions. For $F_{a p p}=0.03$, breaking starts near the top and left sides at $t=6,384$. Failure cascades and last to be disconnected are edges near the bottom right corner where the least amount of force is felt. The network totally collapses at $t=6,602.4375$. No connections remain and the network elongates and moves diagonally following the net force as shown by the red arrows in the network movement panel. 


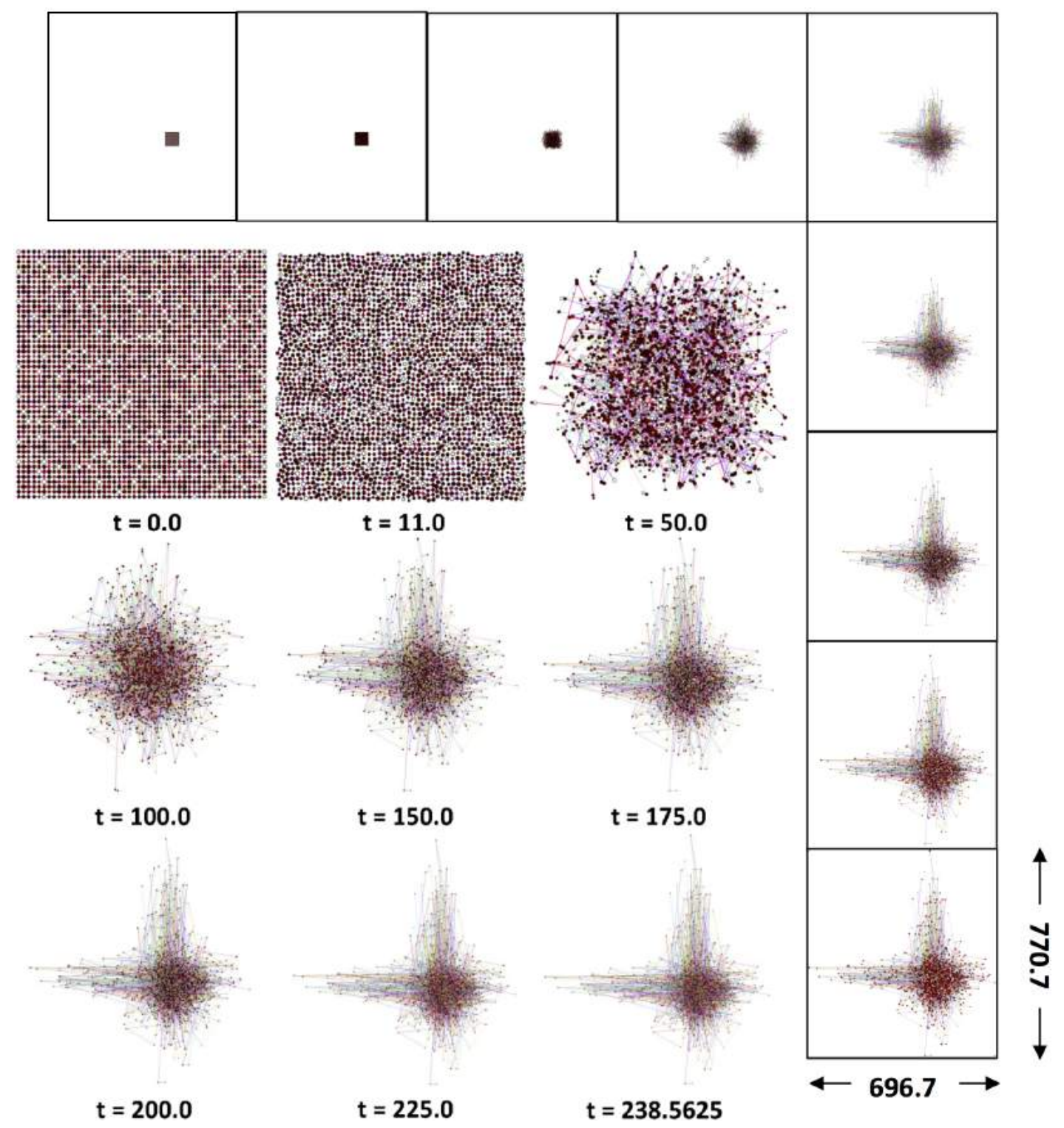

Figure 6.8: Evolution of a lattice network with random force directions. For $F_{\text {app }}=0.03$, breaking starts at $t=11$ at random positions. The network reaches its equilibrium structure at $t=238.6$ with only $0.496 \pm 0.007$ of the edges disconnected. The top and right panels show the relative expansion of the network in space. 
the greatest amount of force. The edges' disconnection pattern follows the direction of the net force which is diagonally towards the most influential node at the bottom right corner. This node-to-node forcing pattern results to a global acceleration that causes the network to elongate diagonally towards the bottom right corner as shown in the network movement of Fig. 6.7. Directing all forces towards the most influential node will globally affect the entire network to move towards the direction of this node.

Fig. 6.13(a) shows the fraction of disconnected edges $f$ as a function of time for all values of applied forces $\left(F_{a p p}\right.$ are values in $\left.[0.0009,1000]\right)$ used when the direction of $F_{a p p}$ in the network is uniform. The behavior of $f$ follows a threshold function similar to an inverse bond percolation model. We find that edges started to fail at different times depending on the $F_{a p p}$ used. The smaller the magnitude of force applied by the nodes, the longer the agents stay in the network.

An abrupt change in the network's state is observed after a small additional force is applied [116]. The lattice network failure observed here does not gradually become more likely but instead there is a transition point during which disconnections are fastest as shown by the disconnection derivative $f^{\prime}$ in the inset of Fig. 6.13(a). Around this critical point, any additonal force causes a sizeable amount of edges to fail, eventually leading to a complete network collapse. But decreasing $F_{a p p}$ can help slow down the disconnections.

\subsubsection{Random force directions}

The directions of the applied forces in a lattice network are random when the influential nodes of the network are positioned randomly in grid space. Some nodes are influenced by only a few nodes while others receive much influence (see Supplementary Section A.3.1.2). The directions of $\left\{F_{a p p}^{k}\right\}$ are assigned at the start of each simulation and are maintained constant. Initial breakings start at random positions in the lattice as shown in Fig 6.8. As a result of assigning this directionality of interactions, the nodes disperse away from the initial center of the network with more nodes moving upward and to the left. This bias towards the upward and right directions is a result of the randomization which positions 68 (2.72\%) more influential nodes to the right and $34(1.36 \%)$ more influential nodes at the top. But this net drift is only $0.11 \%$ of the drift when forcings are uniform. The random placement 
of the influential nodes in the network aids in preserving some of the connections in the network and prevents large magnitude changes in the global position of the network.

The preservation of links is reflected in Fig. 6.13(b) which shows the fraction of disconnected edges $f$ as a function of time for all values of applied forces. Similar to lattice networks with uniform force directions, the failure of the edges has a characteristic phase transition. But we find that randomizing the force directions in a lattice network results to a more resilient network. For all $F_{a p p}$, lattice networks of this type preserve more than half of its edges with only $0.496 \pm 0.007$ disconnected edges.

\subsection{Erdos-Renyi network}

Examining the evolution of a random network or an ER network remains significant even after real-world networks were found to be otherwise. Systems like the power grid or the neural network of C.elegans are best described as evolving networks [122]. The development of such evolving networks are characterized by not only a powerlaw but also by exponential degree distributions [122]. Preferential attachment, aging effects and constraints in the growth of a network can in the long run lead to a transition to an exponentially decaying degree distribution. It is in this regime of the dynamic evolution of a network that findings regarding the resilience of an ER network may be of importance. The ER networks considered here have the same number of edges (i.e. 4, 900 edges which is approximately equal to a $0.2 \%$ probability of nodes being connected) as in the lattice networks to allow for comparisons. ER networks with higher fraction of nodes will have a bigger giant cluster leading to a delay of breakdown. All other results presented in this section are robust to number of edges in the Erdos-Renyi network.

\subsubsection{Uniform force directions}

Though connections among nodes in an ER network are random, forcing directions can be made as uniform as possible by preferring specific positions in placing influential nodes (i.e. bias in vertical interactions such that nodes are influenced only by those below them) as discussed in Supplementary Section A.3.2.1. 
The first disconnections are the result of nodes breaking away from the more influential nodes at the bottom as illustrated in Fig. 6.9. Being influential allows an agent to affect more nodes but this can also mean that their connections are more likely to be short-lived. A cascade of disconnections from the more influential nodes is observed at just a short period of time. But there are some connections that persist far longer than others. As the network evolves, the individual node-to-node force interactions lead to a more dispersed network with the nodes moving outward with a slight drift downwards towards the influential nodes.

\subsubsection{Random force directions}

In randomizing the force directions in an ER network, we are distributing the influentials in grid space. For such case, nodes are influenced by other nodes regardless of their position (see Supplementary Section A.3.2.2). Since influence is distributed, there is no bias in the global movement of the network and breaking also starts in random positions as shown in Fig. 6.10. Similar to the uniform force directions case, a large amount of edges fail initially (79.6\% of edges). In a span of 100 iterations, $97.0 \%$ of the edges have already failed. The remaining $3.0 \%$ of the edges persists longer and disconnections slow down until the network totally collapses. Randomizing force directions also leads to a one order of magnitude decrease in the global dispersion of the nodes from the center of the network.

\subsection{Barabasi-Albert network}

Most real world networks from cellular and genetic networks to social, citation, infrastructure and transport networks exhibit scale-free degree distributions that is well represented by BA networks [122, 123, 124, 125]. We investigate here how the direction of forces affect the fragmentation of networks having scale-free degree distributions.

\subsubsection{Uniform force directions}

One of the key attributes of BA networks is the presence of a hub responsible for most of the connections in the network. In the uniform force directions case 


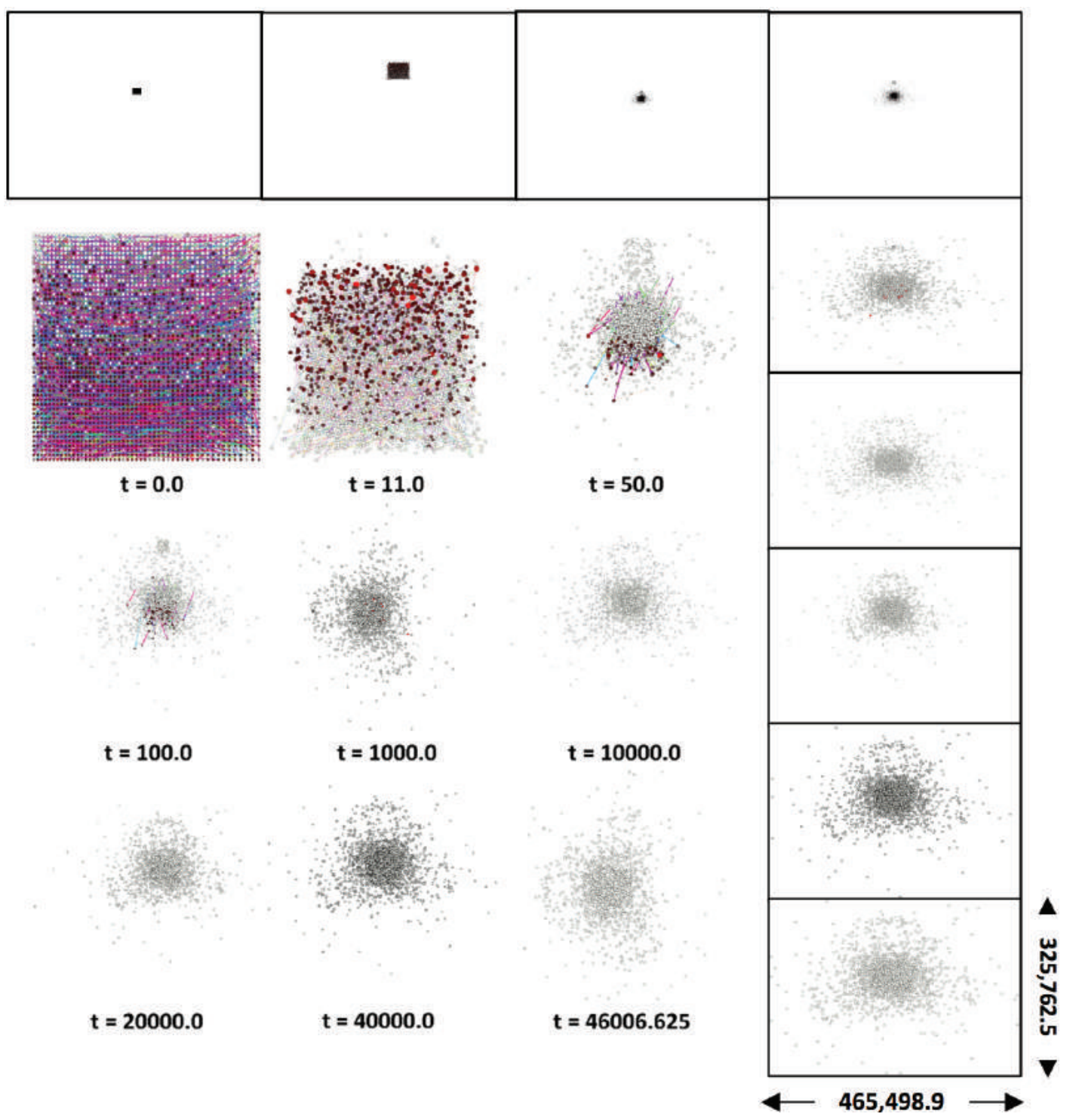

Figure 6.9: Evolution of an Erdos-Renyi network with uniform force directions. For $F_{a p p}=0.8$, breaking starts with nodes disconnecting from the influential nodes at the bottom resulting to $77.61 \%$ of the edges to break instantly. By $t=100,95.73 \%$ of the edges have disconnected. The remaining edges take far longer to disconnect. The top and right panels show the relative expansion of the network in space. 


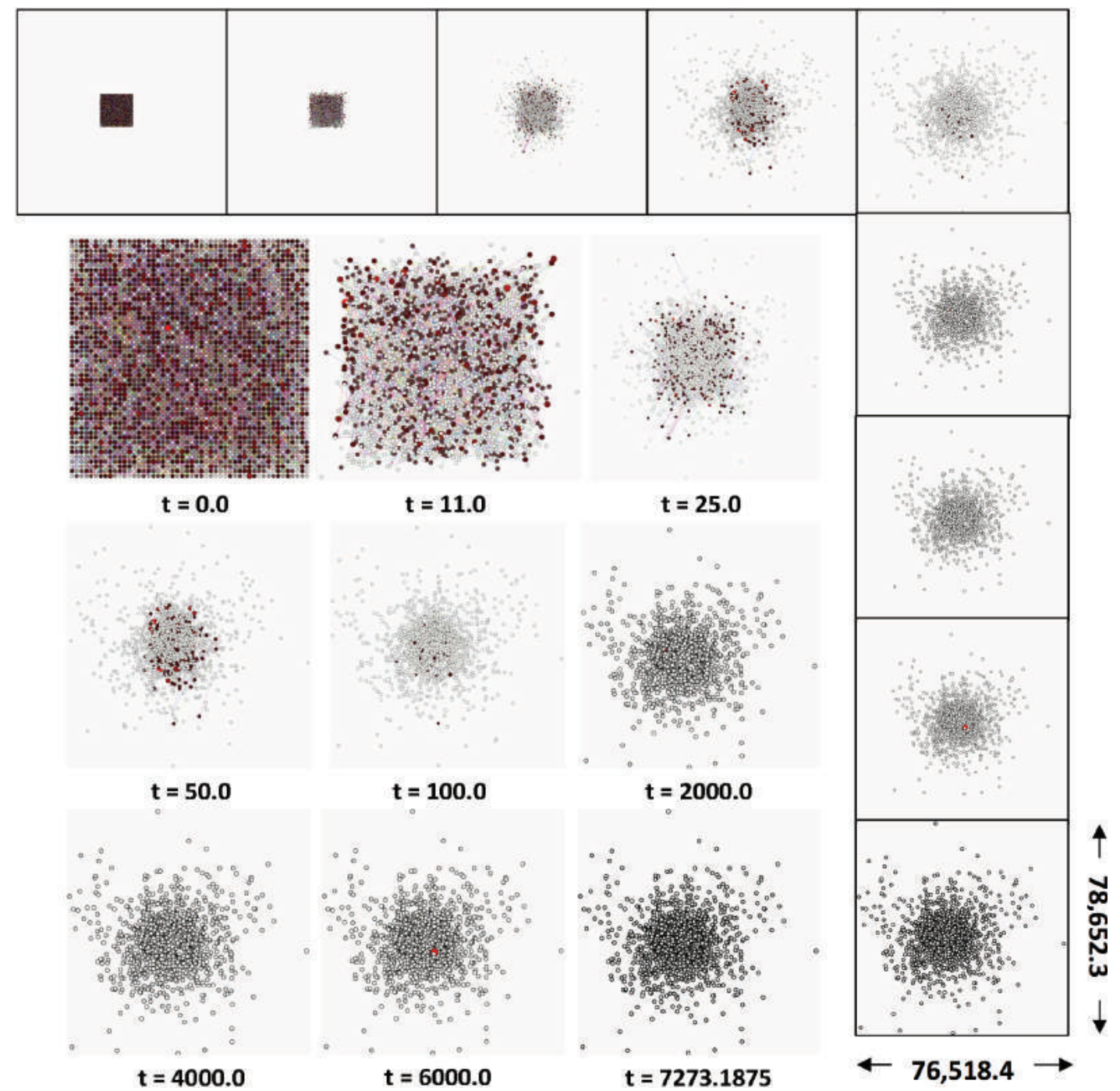

Figure 6.10: Evolution of an Erdos-Renyi network with random force directions. For $F_{a p p}=0.8$, breaking starts at random positions. Initial disconnections account for $79.6 \%$ of the edges. By $t=100,97.0 \%$ of edges have disconnected. The remaining $3.0 \%$ breaks slower until the network completely collapses at $t=7,273.1875$. The top and right panels show the relative expansion of the network in space. 
shown here, most of the edges are directed away from the hub while all others are connected by preferring a certain direction as further illustrated in Supplementary Section A.3.3.1. Another uniform directions case where edges are directed towards the hub (Supplementary Section A.4) was also considered and found to have similar results.

Since the hub contains most of the connections and controls the movement of most nodes, the network eventually becomes completely fragmented but $3.3 \%$ of the edges take far longer to disconnect as shown in Fig. 6.11. The hub, being connected to a large number of nodes scattered throughout the spatial grid network, is pulled in all directions triggering a cascade of disconnections until no connection remains in the network regardless of $F_{a p p}$ as shown in Fig. 6.13(e). Connections to nodes farthest to the hub are the first ones to break and are left far in space from the cluster of nodes as depicted in the network movement in Fig. 6.11.

\subsubsection{Random force directions}

Randomizing force directions meant keeping the number of hub edges that are directed inward and outward comparable. This allowed nodes other than the hubs to have paths to almost all nodes as discussed in Supplementary Section A.3.3.2. However, the presence of a hub still led to complete network fragmentation as shown in Fig. 6.13(f).

Redistribution of forces and addition of paths among the network nodes were not enough to overcome the influence of the hubs. This holds regardless of the position of the hubs in grid space (Supplementary Section A.4). Although preservation of connections was not achieved by randomizing force directions, nodes in this case are spatially closer. Random force directions kept the nodes closer by $34.1 \%$ in terms of area.

We discuss in detail in the succeeding sections how this framework can be applied to an actual bus transport network. 

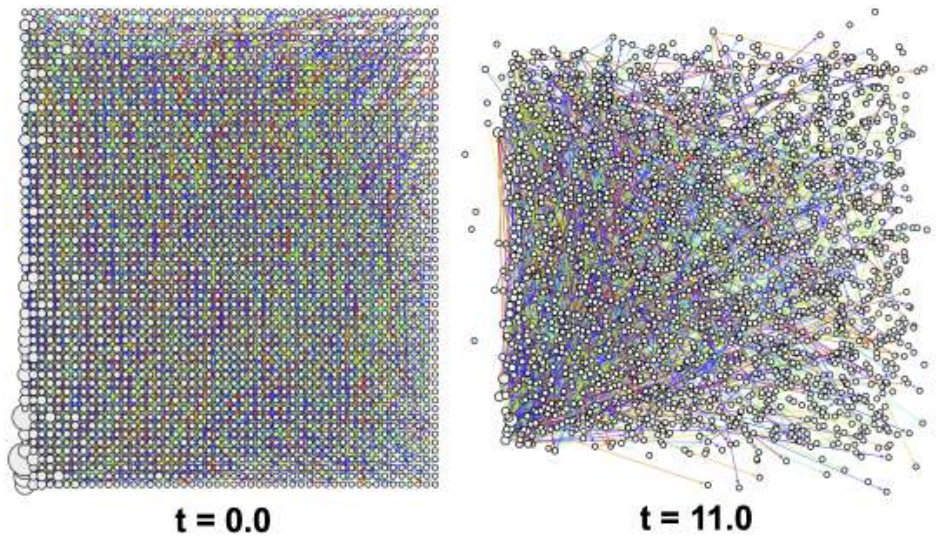

$\mathbf{t}=\mathbf{3 2 . 0}$
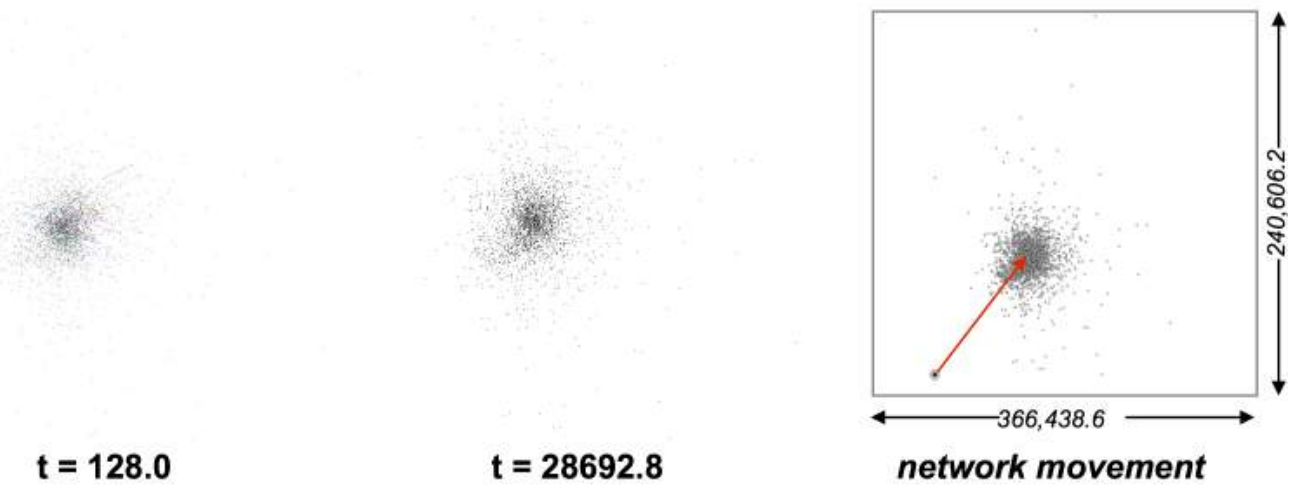

Figure 6.11: Evolution of a Barabasi-Albert network with uniform force directions. For $F_{a p p}=0.8,75.9 \%$ of the edges fail instantly and by $t=128.0,96.7 \%$ of the edges has disconnected. The remaining edges have all disconnected by $t=28,692.8$. 


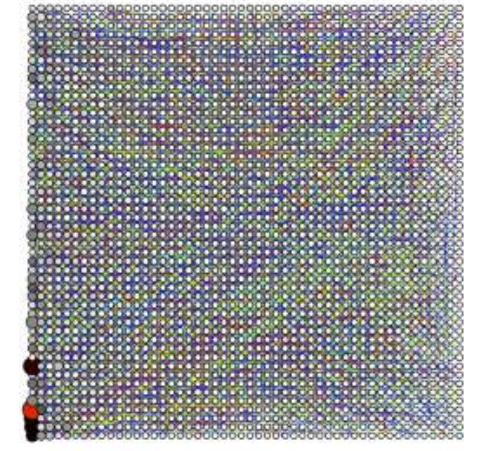

$t=0.0$

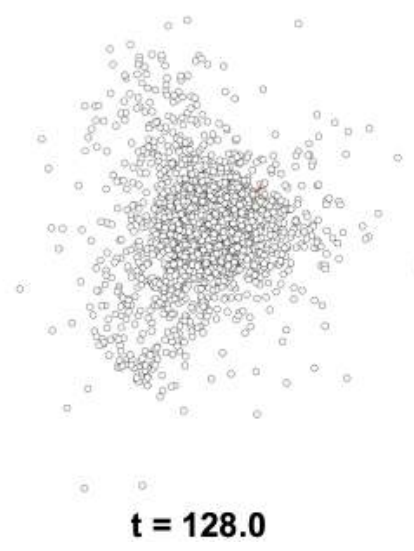

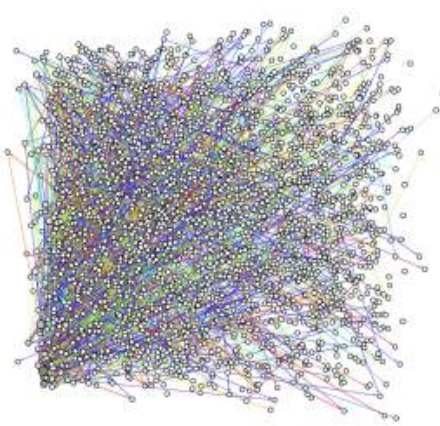

$t=11.0$

$\mathbf{t}=\mathbf{3 2 . 0}$

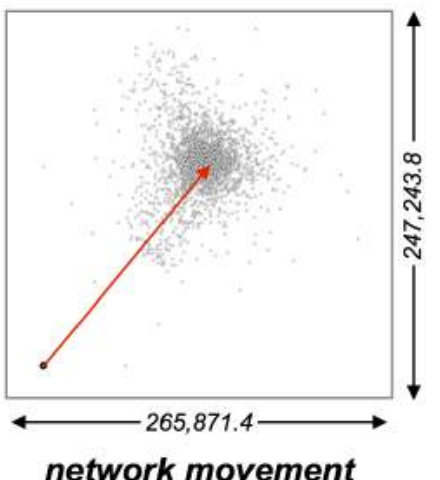

Figure 6.12: Evolution of a Barabasi-Albert network with random force directions. For $F_{a p p}=0.8,75.9 \%$ of the edges fail instantly with only $1.9 \%$ of the edges remaining by $t=128.0$. The network completely fragments at $t=22,773.6$. 


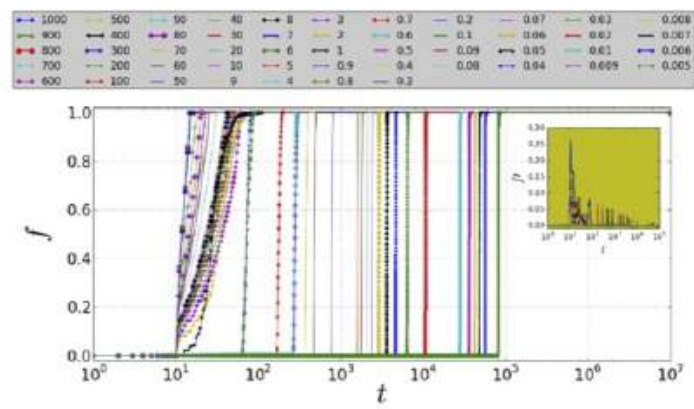

(a) Lattice network with uniform force directions

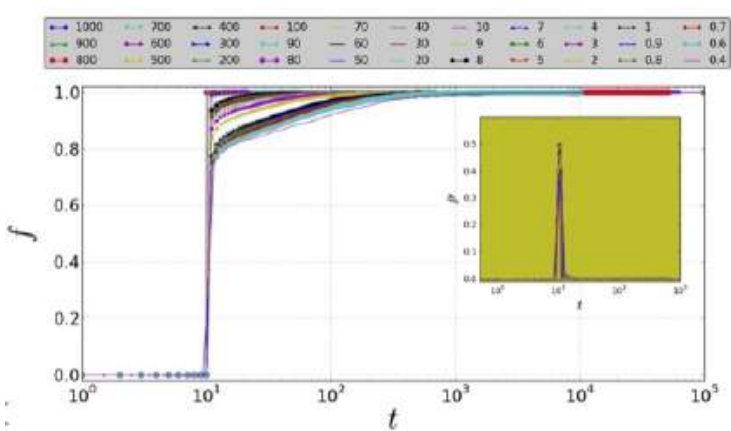

(c) ER network with uniform force directions

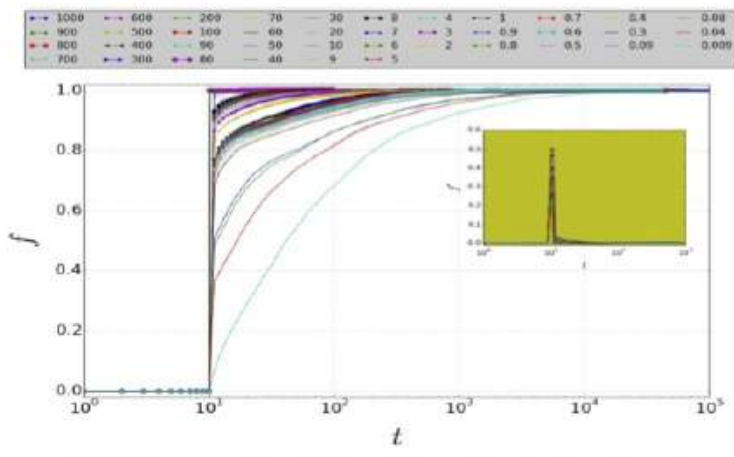

(e) BA network with uniform force directions

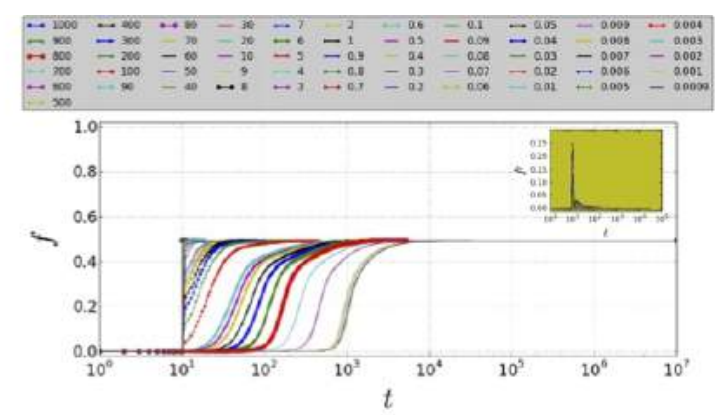

(b) Lattice network with random force directions

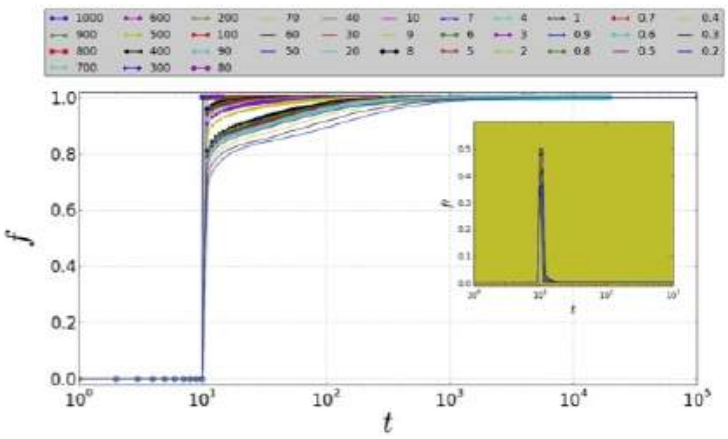

(d) ER network with random force directions

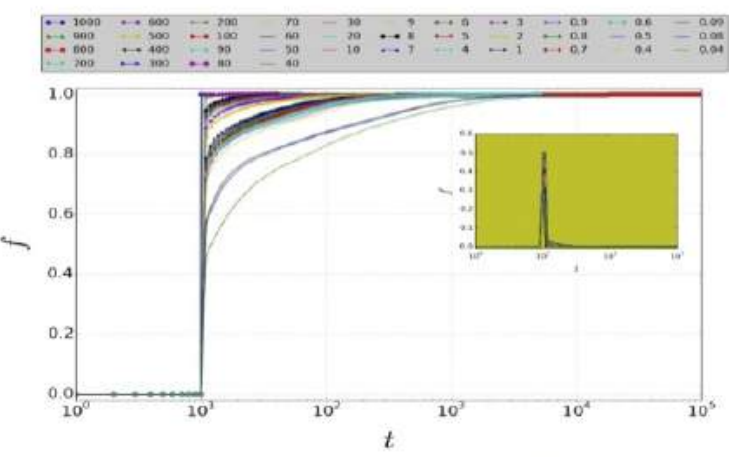

(f) BA network with random force directions

Figure 6.13: Fraction of disconnected edges $f$ in grid networks. (a) Lattice networks with uniform force directions totally collapse while for those with (b) random force directions, $0.503 \pm 0.006$ of the edges disconnects. (c-d) Regardless of the magnitude and directions of $\left\{F_{a p p}\right\}$, an ER network experiences total system collapse. (ef) BA networks completely fragment regardless of the magnitude and directions of $\left\{F_{a p p}\right\}$. The insets show the derivative of $f$ or the disconnection rate of the edges. The rate of disconnection $f^{\prime}$ at the transition point decreases with $F_{a p p}$ in all networks. For lattice networks, randomizing force directions further decreased the rate of disconnections. 


\subsection{Dependence of the preservation of links on the randomness of the force directions}

We have so far explored networks with force directions that are either completely uniform or completely random. We further examine the effect of the force pattern by combining uniform and random force directions in the lattice network. A fraction $p$ of the edges had force directions that were randomized while the remaining edges experienced forces that had the same vertical and horizontal directions.

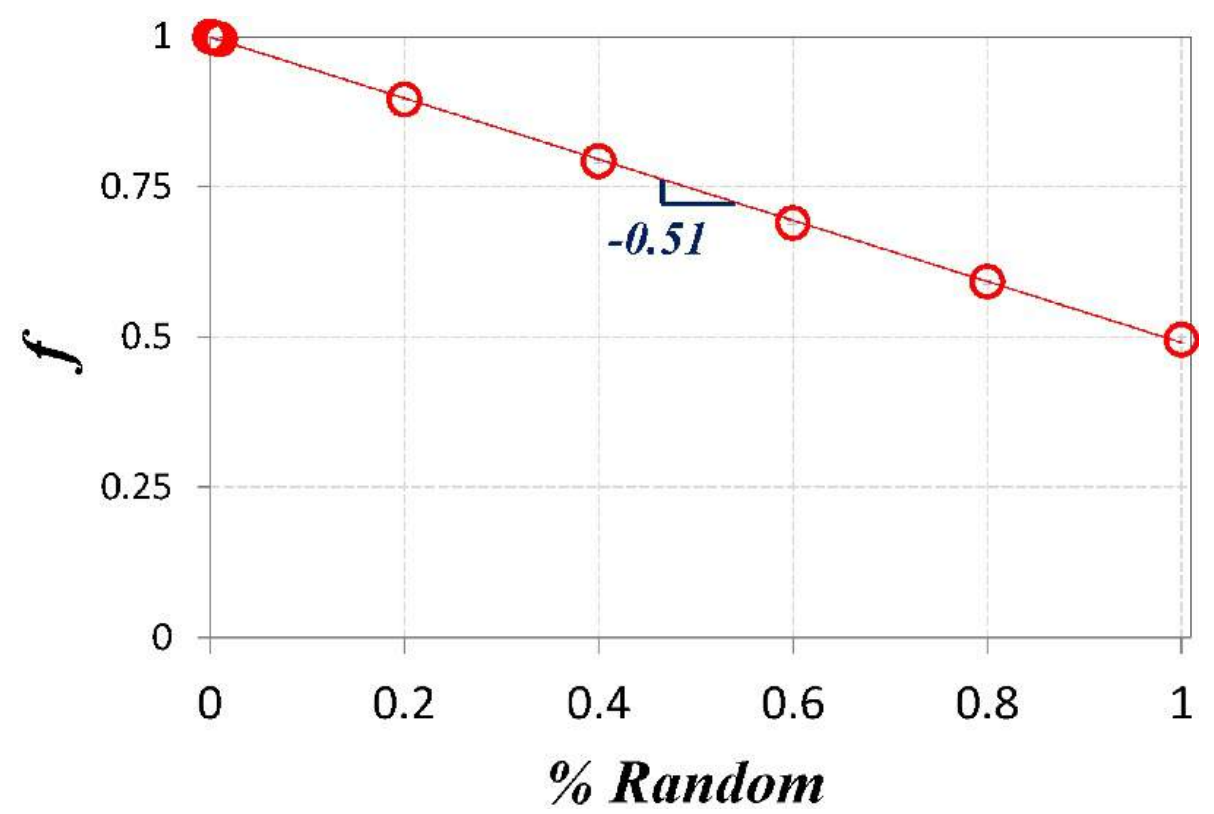

Figure 6.14: The final fraction of disconnected edges in a lattice network with varying degree of force directions randomness. The relationship is found to be linear with a slope of -0.51 .

From Fig. 6.14, the fraction of disconnected edges in the final network is seen to follow the relationship $f=1-0.51 p$. This very close linear fit warrants further investigation. An analytical study of this behavior can reveal the origin of this linear relationship beween the probability of force direction randomness and fraction of intact edges. A step to this direction has recently been taken by Zhao in his study describing an analytical framework predicting the relative sizes of residual node clusters in uncorrelated directed random graphs [126].

The lattice network contains more disconnected edges as the force directions 
become more random. With the introduction of increased randomization, forces in the network become less concentrated causing edge failures to be more spatially distributed. This suggests that a force pattern that is most dissimilar to the connectivity pattern of the network may be the most ideal in constructing a resilient network. In the lattice network, nodes are connected uniformly such that only nearest neighbor connections are allowed. Subsequently, completely random force directions prove to be the best choice in preventing significant network fragmentation.

This provides an explanation as to why the final number of edges in the ER and BA networks were not found to depend on force directions. Since both long-range and short-range links are allowed and edges can be of varying spatial angle, the uniform force directions case is not of totally uniform edge directions. This results to the presence of some degree of randomization even in the uniform force directions pattern case.

\subsection{Lifetime of grid network}

Fig. 6.15 shows the lifetime of the networks as a function of $F_{a p p}$. The evolution of all the networks considered with deformation memory follows Basquin's Law at the regime below the force limit, $F_{\text {limit }}$, with lifetimes having a power law behavior with respect to $F_{\text {app }}$. When $F_{\text {app }}>F_{\text {limit }}$, instantaneous breakings dominate causing $t_{f}$ to deviate from a power law behavior [119]. $F_{\text {limit }}$ is approximated by setting $f_{0}=0$ and looking for the force magnitude for which (1) no fragmentation occurs for 5,000,000 iterations (at least one order of magnitude above the lifetimes of the networks considered), and (2) $t_{f}$ decays rapidly with increasing $F_{a p p}$ [121]. For lattice networks with uniform force directions, $F_{\text {limit }}=1.21$ while for the case with a fraction of random force directions, $F_{\text {limit }}$ is smaller at $0.09 \pm 0.03$. The ER networks followed Basquin's Law at forces below 103 for both cases. $F_{\text {limit }}$ of the BA networks, on the other hand, is 111 for the uniform force directions case and 55 for the random force directions case.

In the work of Carmona et al. [119], they showed that it's possible to change the Basquin's exponent, $\gamma$, up to a magnitude of 0.40 by using different breaking thresholds. Using the same method they employed in calculating for Basquin's exponent, we demonstrate that $\gamma$, even for the same breaking rules, can be tuned by 


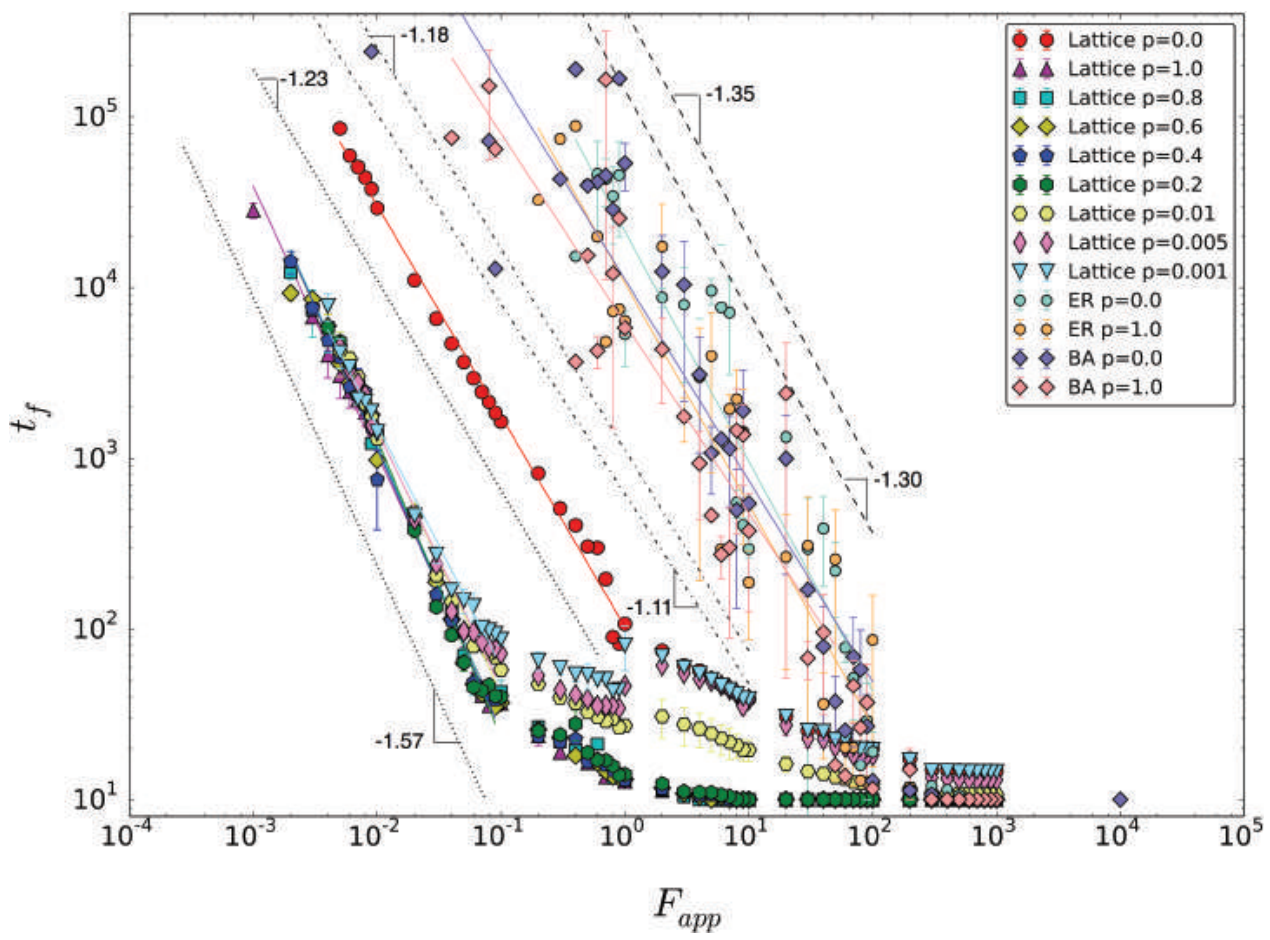

Figure 6.15: Lifetime of spatial grid networks. Dashed lines illustrate the Basquin's exponent of the networks.

either altering (1) the network structure or (2) the force directions pattern.

For the same lattice network, a change in $\gamma$ of magnitude 0.31 was achieved by randomizing the force directions of even only 0.1 of the links. For the ER networks, a minimal change of 0.05 in $\gamma$ was found, providing added evidence that the uniform force directions method was not successful in "regularizing" the structure of the random network. A small change of 0.07 in $\gamma$ was also found for BA networks. The presence of both long-range and short-range links in the ER and BA networks causes a minimal change in the characteristic exponent of their lifetimes when force directions are changed. In both networks, however, the random force directions case had a smaller $\gamma$ magnitude which implies that on average, network fragmentation can be delayed by randomizing force directions.

The fragmentation process proceeds in bursts with a universal scale-free distribution. The bursty dynamics of edge breaking in the micro-level when memory is incorporated leads to the macro-scale power law distribution of network lifetime $[127,119]$. We illustrate this in Fig. 6.16 where the waiting time $T$ between breakings has a power-law distribution $P(T)$ and $<T>\sim\left(F_{a p p}\right)^{-(1+\gamma)}$. Having networks 


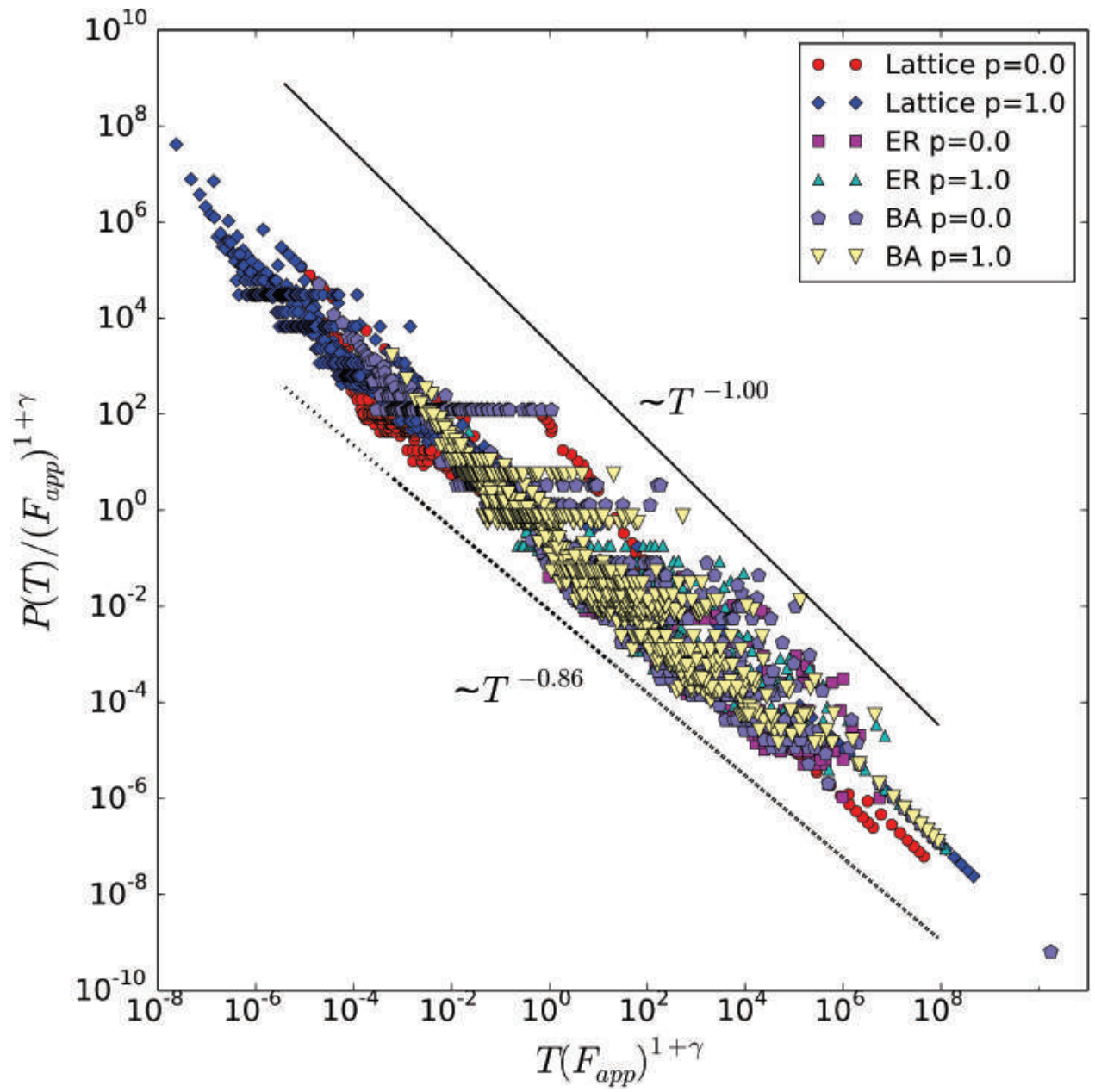

Figure 6.16: Damage sequence waiting time. The macro-scale network lifetime results from microscopic scale-free waiting times between bursts. The lines shown are the range of exponents of the power-law distribution. 


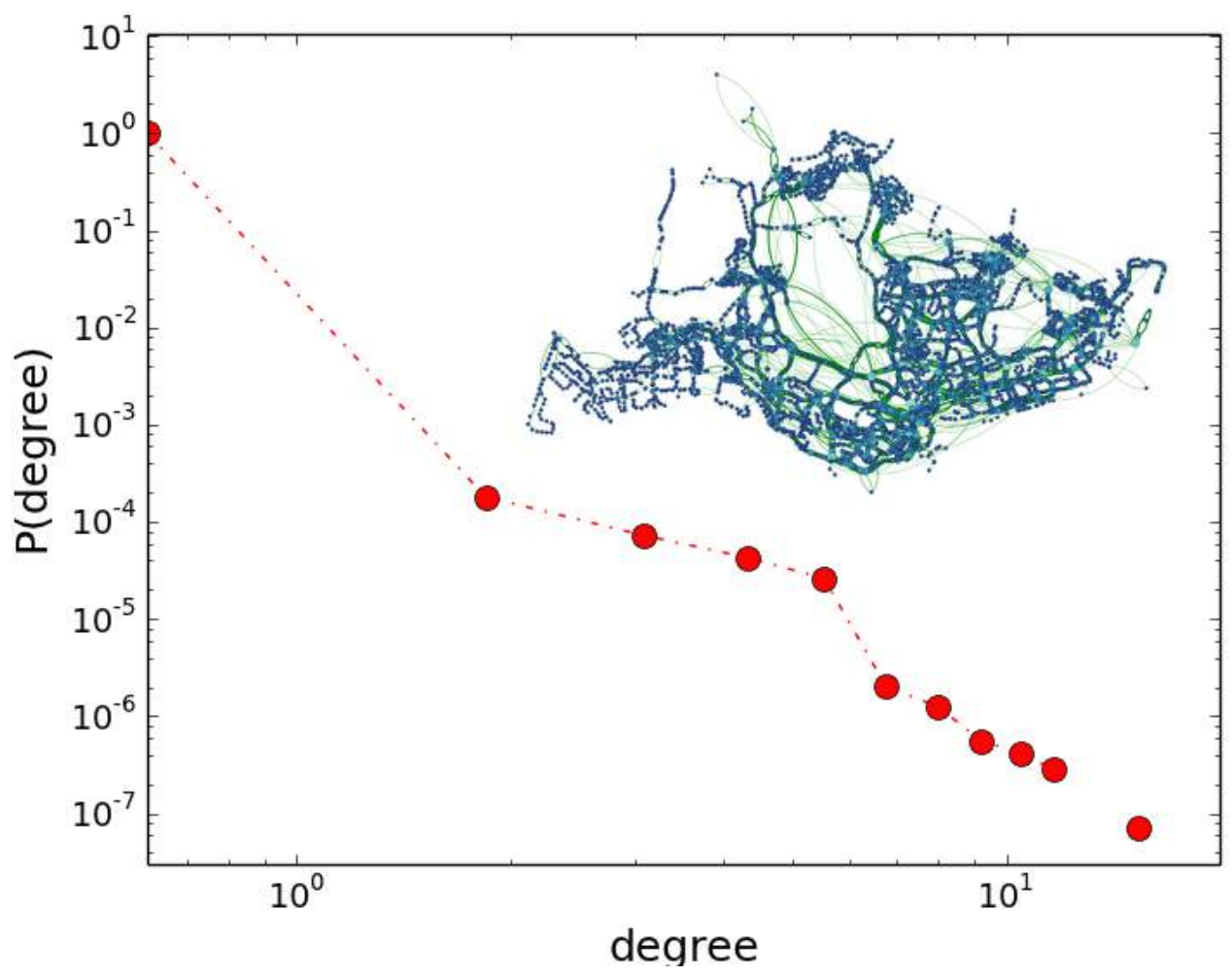

Figure 6.17: Degree distribution of Sinagpore bus network.

of very different degree distributions all demonstrating the same feature hints to the universality of this evolutionary dynamics and scale-free network lifetime.

\subsection{Application to a real-world transport network}

Continuous use of the transport network at high throughputs can lead to a sizable number of edges to break causing a network collapse. Some of the factors that influence the disconnection of links in evolving networks are force magnitudes, duration of time these forces are applied, and how nodes are connected and routes are directed in the system. Choosing the appropriate directionality of station connections (i.e. train or bus routes) can optimize the distribution of load in the system and can consequently make a network more resilient to edge failures [128].

We illustrate here how our framework can be applied to a real-world network. We show our dynamical model of network evolution with an applied force between its nodes (i.e. commuter throughput) in the context of a generic Barabasi-Albert network. Most real world networks including infrastructure and transport networks [129, 130, 131] have broad-tailed degree distributions which are characteristic of 


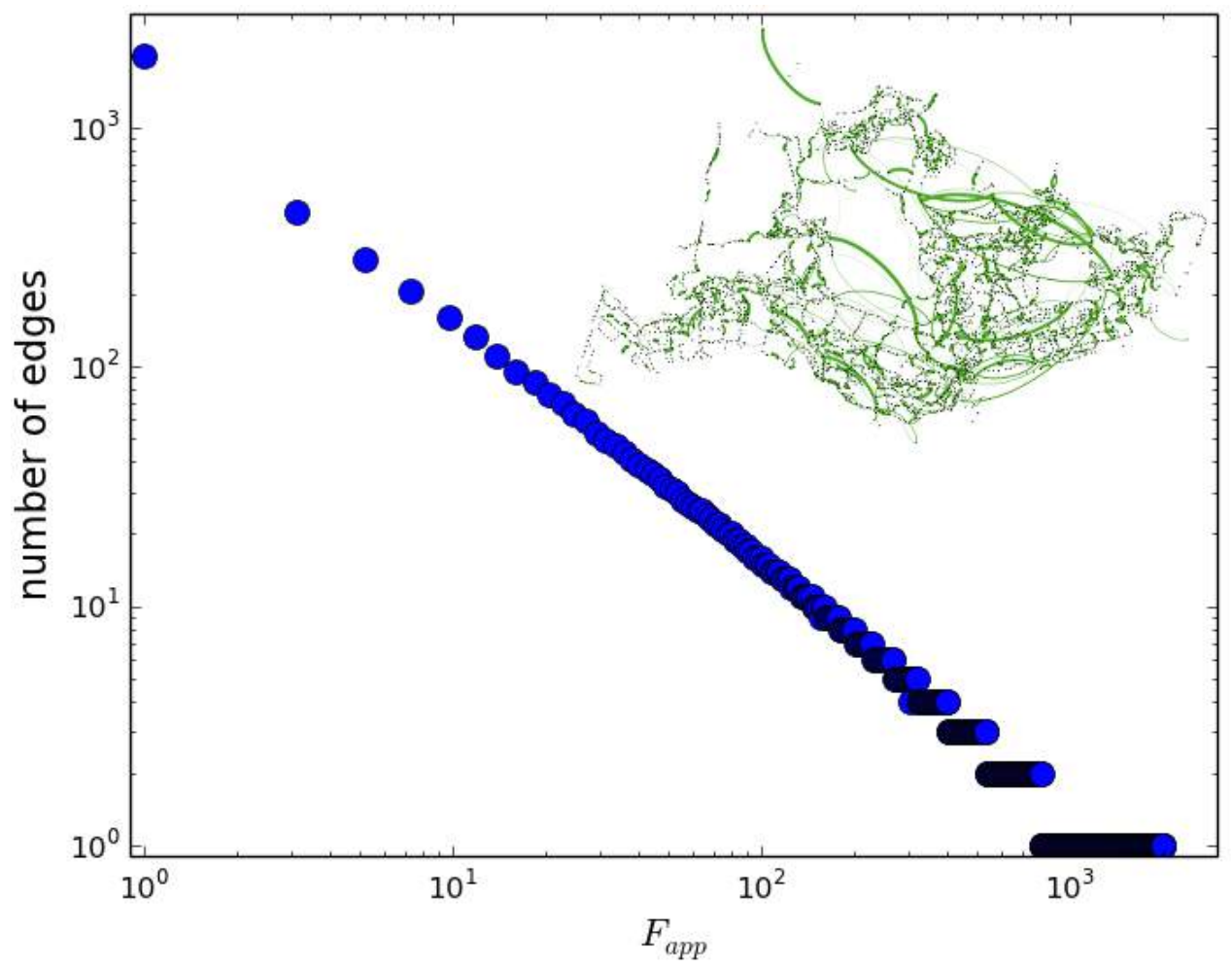

Figure 6.18: Number of edges for each Fapp. Inset shows bus network edges weighted by $F_{a p p}$.

Barabasi-Albert networks. In Fig. 6.17, we show the broad-tailed degree distribution of the Singapore bus network.

The dynamical model of network evolution which can be used to explore the resilience of a network under different force magnitudes and directions can be applied on the actual bus network of Singapore. The nodes of the network as shown in the inset of Fig. 6.17 are the bus stops and an edge is constructed between bus stop a and b if there's at least one bus service that travels from bus stop a to bus stop b. We use the actual (longitude, latitude) locations of the bus stops to position them in the grid network.

In the absence of additional data, it can be assumed that the demand capacity of the links is constant across the entire bus grid network. Hence, in simulating the evolution of the bus grid network, the elongation thresholds are the same for all edges. For the case of the Singapore bus network, it has been shown that the origin-destination (OD) travel demand is a scale-free distribution [132]. This means that there are only a few OD-pairs that are highly utilised while most of the pairs are rarely used [132]. We can think of the travel demand as the applied force 
experienced by every connected nodes. The applied forces can then made to vary according to

$$
\text { number of edges }=2000 x\left(F_{\text {app }}\right)^{-1}
$$

which is similar to the relationship between the number of OD pairs and number of journeys per OD pair found in [132]. The assignment of $F_{a p p}$ according to Eqn. 6.8 is done by randomly choosing from a biased list of edges with the probabilities of selection proportional to the number of bus routes that includes each OD pair. The OD pair edge used the most has higher probability of being assigned the largest $F_{a p p}$. The number of edges assigned for each $F_{a p p}$ is shown in the Fig. 6.18.

We vary the directions of the edges to investigate the resilience of the Singapore bus transport network under different bus route directions. The force directions along the edges considered are (a) the actual bus route directions, (b) force directions towards nodes with higher degree, (c) force directions away from nodes with higher degree, and (d) random force directions as shown in Fig. 6.19.

For the same force magnitudes applied to all edges, the Singapore bus network lasted longest when the force directions were assigned randomly (Fig. 6.23) since this method reduces the influence of the higher degree nodes. The next most effective strategy in delaying complete network fragmentation is when forces are directed away from the node (Fig. 6.22), followed by the actual bus directions (Fig. 6.20). Finally the least resilient network is when forces are directed towards the higher degree nodes (Fig. 6.21) in which case we are increasing the influence of the hubs even more, thereby hastening network collapse.

Using the network lifetime $t_{f}$ given by the model as a measure of resilience, we can probe the effect of specific changes in bus route directions on the robustness of the transport network. Coupled with travel demand models, this will aid urban planners in constructing a more robust and resilient transport network.

\subsection{Summary and Conclusions}

A considerable number of studies have been made on the the evolution of networks in space $[1,104,105,106,107,108,109]$. But most of these does not consider what happens to the lifetime of networks once we give them a memory of their 


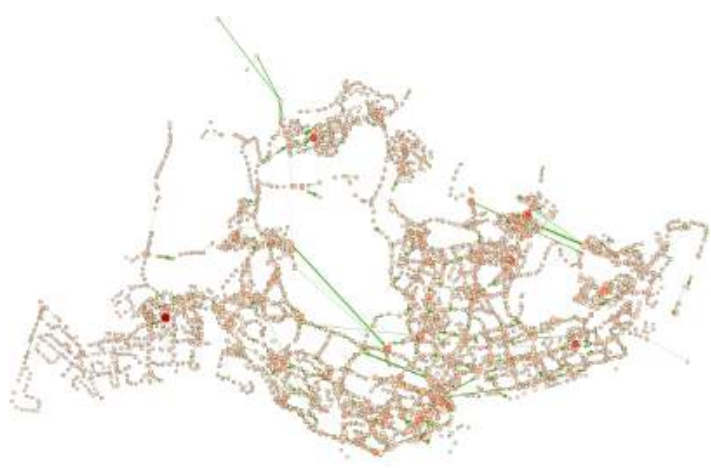

(a) actual bus directions

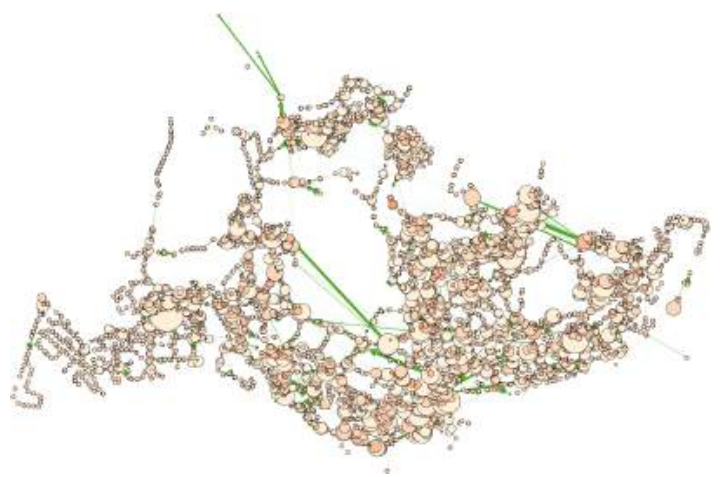

(c) away from higher $k$ node

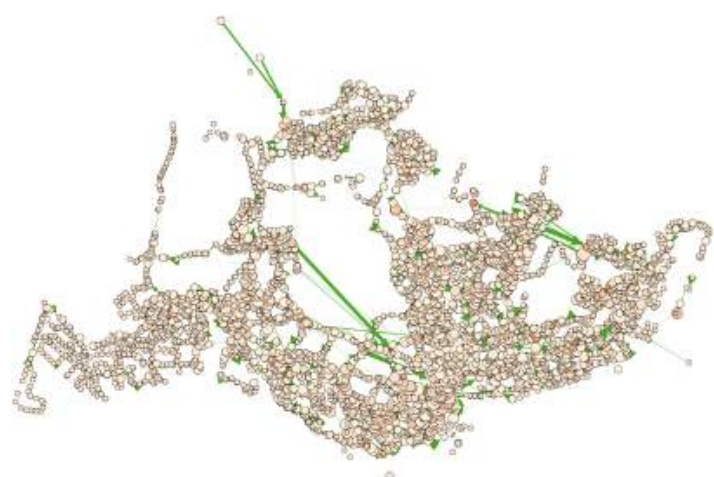

(b) towards higher $k$ node

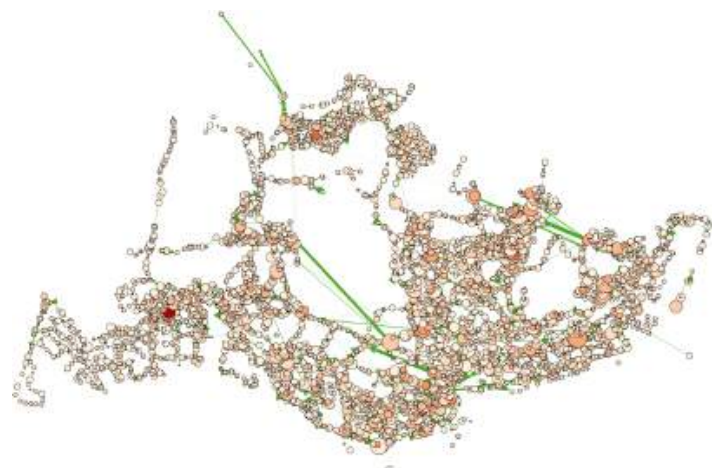

(d) random directions

Figure 6.19: Force directions in Singapore bus network. Force directions considered are (a) actual bus directions, (b) towards nodes with higher degree $k$, (c) away from nodes with higher degree $k$, and (d) random directions. Nodes are colored according to their in-degrees and their sizes are based on their out-degrees. Edges are weighted according to $F_{a p p}$.
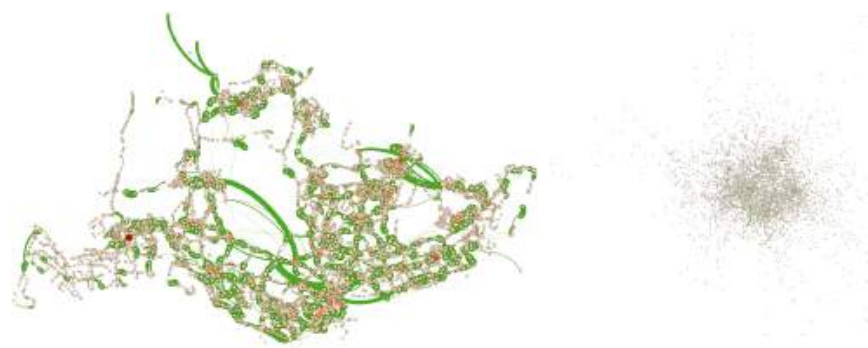
(a) $\mathrm{t}=0.0$
(b) $\mathrm{t}=11.0$
(c) $\mathrm{t}=31.125$

Figure 6.20: Singapore bus network evolution with actual bus routes as force directions. The network completely fragments at $t=31.125$. Nodes are colored according to in-degrees and their sizes are based on out-degrees. Edges are weighted according to $F_{a p p}$. 


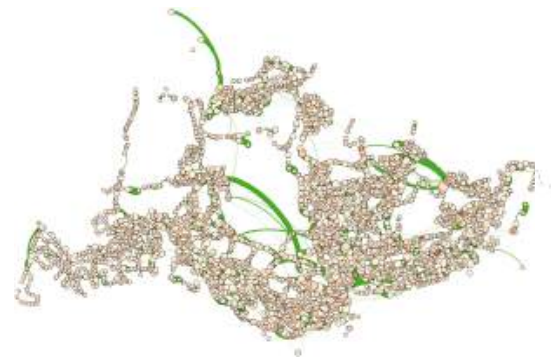
(a) $\mathrm{t}=0.0$
(b) $\mathrm{t}=11.0$
(c) $\mathrm{t}=26.0625$

Figure 6.21: Singapore bus network evolution with forces towards nodes with higher degree. The network completely fragments at $t=26.0625$. Nodes are colored according to in-degrees and their sizes are based on out-degrees. Edges are weighted according to $F_{a p p}$.

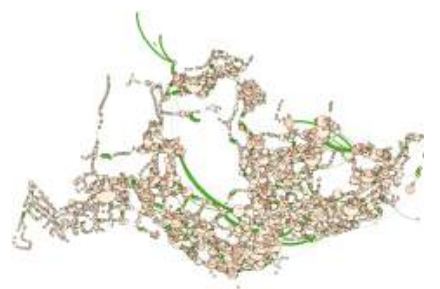
(a) $\mathrm{t}=0.0$
(b) $\mathrm{t}=11.0$
(c) $\mathrm{t}=32.0$
(d) $\mathrm{t}=37.4375$

Figure 6.22: Singapore bus network evolution with forces away from nodes with higher degree. The network completely fragments at $t=37.4375$. Nodes are colored according to in-degrees and their sizes are based on out-degrees. Edges are weighted according to $F_{a p p}$.
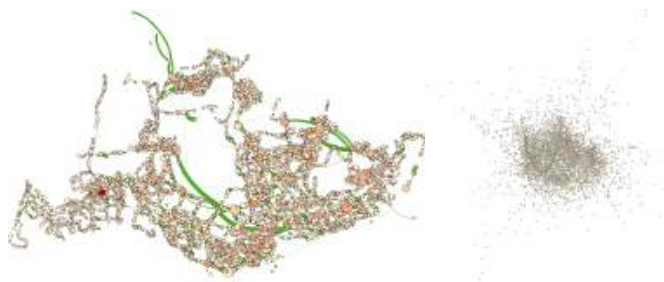
(a) $\mathrm{t}=0.0$
(b) $\mathrm{t}=11.0$
(c) $\mathrm{t}=32.0$
(d) $\mathrm{t}=52.75$

Figure 6.23: Singapore bus network evolution with random force directions. The network completely fragments at $t=52.75$. Nodes are colored according to indegrees and their sizes are based on out-degrees. Edges are weighted according to $F_{\text {app }}$. 
deformation history. Modelling the evolution of a network through time as a result of the interplay of forces within the network and embedding the network in Eucledian space can provide a more realistic view of how network topology and structure change. We can imagine a network as a set of nodes exerting a force on each other when they are connected. In this study, we investigated the evolution of such a network embedded in space. Examples of these are grids, transport networks, archaeological networks or even any kind of network embedded in relational space. By following the evolution of networks in space and time, we investigated how damage accumulation or deformation memory affects the lifetime of networks.

In developing our model, we take inspiration from studies of fracture and fatigue in physical systems. Fracture can be imagined not just in the context of materials. It also happens in other networks like road, protein, power grid and neural networks. Nodes here are not molecules or atoms like in physical networks but macroscopic entities that may possibly be observed only through their interactions. Force and loading that lead to fracture exist in these systems in the form of vehicular or energy flows or strength of interactions between nodes. In all these classes of systems, one thing is true. Memory is important. Whether a particular link will fail or not depends not only on the instantaneous deformation resulting from the force a node applies on another at the current time, but also on how it remembers past forces it has experienced. This damage accumulation term introduces memory over the loading history of the system.

Following the work of Carmona et al. [119], we investigated here the eventual network structure of a directed spatial grid network with lattice, ER, or BA connections that result from forces that the nodes apply on each other along the edges linking them. The method presented here is general and can be used to probe other kinds of spatial networks as illustrated in Supplementary Section A.5.

For a lattice network, uniform edge directions which result to force influence of nodes to systematically increase in spatial grid lead to an inevitable network collapse for all values of $F_{a p p}$ in a network having a memory factor of 0.05. Such edge directions pattern results to the maximum amount of force to be applied to nodes of lesser force influence (i.e. nodes with no outgoing edges and hence do not apply force on any node) leading to a network of completely non-interacting components. But randomly positioning the influential nodes (i.e. nodes with more 
outgoing edges than incoming edges) results to a more distributed force which helps in preserving network relationships and keeps the giant cluster intact as shown in Supplementary Fig. A.10 and Fig. A.11. A lattice network is hence more resilient when the force sources are distributed randomly in the network instead of uniformly arranging them.

However in the same lattice network, when forces are uniformly directed, the start of breaking happens at a later time (Supplementary Fig. A.10), at which point, nodes have already moved far from their initial positions. But as seen in the rate of disconnections, once breaking has begun, transitions are slower for networks with random force directions. This is true for all values of applied force which shows that the final topological and connection configuration of a lattice network is dependent on the force directions, but not on the magnitude of applied forces.

For an ER network, the transition to its fragmented state is seen to be faster than in a lattice network (Supplementary Fig. A.10). The magnitude of $F_{a p p}$ also fails to extend the start of network collapse unlike in lattice networks wherein lowering the magnitude of the applied force makes the network intact for longer periods of time. Here, a significant amount of the edges $(0.79 \pm 0.12)$ disconnects and the size of the largest cluster suddenly drops at the early part of the simulation (Supplementary Fig. A.12) for all the force magnitudes considered. On the other hand, for edges with higher thresholds, decreasing $F_{a p p}$ can help extend their lifetime.

The final composition of an ER network is also found to be independent of the force directions. For both the uniform and random forcing direction cases, no edge or link between any node is preserved. If we base the resilience of an ER network on its final topological state, no improvement in the network's resilience can be achieved by changing the interconnection pattern of the network unlike that observed in the lattice network.

Similar to the ER network, a BA network also completely fragments regardless of force directions. This results from the presence of hubs responsible for most of the connections in the network and thereby causing forces to be more concentrated. When looking at the fraction of disconnected edges (Fig. 6.13) and the number of clusters (Supplementary Fig. A.10), the BA network exhibits the same behavior as an ER network primarily because both contain a combination of long-range and short-range links. However, examining the size of the largest component reveals 
that the presence of hubs as in a BA network (Supplementary Fig. A.13) slows down the fragmentation of the largest cluster in the early stages of fast breakings as compared to an ER network (Supplementary Fig. A.12).

Decreasing the magnitude of forces slows down fragmentation regardless of network structure, but is only successful in delaying the onset of fragmentation in lattice networks. For lattice networks, an increase in the randomization of force directions can further lead to a decrease in the rate of fragmentation. Changing the direction of forces has no effect in the rate of fragmentation for both ER and BA networks providing an indication as to why their final state were the same regardless of force directions.

We find that aside from the magnitude of the forces applied by the nodes on each other, the force directions pattern applied along the edges of the network determines the time it takes for the system to reach the critical transition point. The resilience of the network as can be observed from how fragmented the eventual network becomes is improved when the force directions in the network is dissimilar to the connection structure of the network's nodes as observed in the case of a lattice network with random forcing directions. However, networks whose force direction pattern is similar to the network connection type was found to exhibit higher tolerance to failure at small time scales with the Erdos-Renyi network being the most efficient when it comes to load distribution.

Under the presence of memory, the lifetimes of the networks defined as the amount of time it takes for the network to reach its equilibrium $f$ are also found to have a power law behavior following Basquin's Law. We further extend the work of Carmona et al. [119] by demonstrating that Basquin's exponent, $\gamma$, can be altered by changing only the force directions in the network without altering the network breaking thresholds. By looking at the waiting time between successive edge failures, we found that in the presence of deformation memory, the failure process proceeds in bursts with a universal scale-free distribution. This microscopic process leads to two kind of macroscopic evolution. Systems undergo a continuous phase transition where partial failure only occurs (i.e. the network does not break up into individual nodes) when the applied force is less than the fatigue limit. However, when the fatigue limit is exceeded, a collapse visible macroscopically occurs. This micro-scale bursty breaking of edges leads to the macro-scale power law distribution of network 
lifetime. This holds true for networks of very different degree distributions hinting to the universality of this mechanism.

\subsection{Future Work}

We have shown in this work the universal analytical relationship of network lifetime and damage sequence waiting time on the magnitude of applied force. The challenge of formulating a rigorous analytical derivation of network lifetime based on the direction of applied forces that we introduced here, however, remains. There is also a need to further understand analytically based on first principles, the effect of both network structure and force directions on the fraction of disconnected edges.

A natural first extension to this work is exploring the mathematical underpinnings behind the close linear relationship of the randomness probability of force directions, $p$, and fraction of intact edges, $f$, of lattice networks found in Section 6.6. Together with this, one can also explore the $p$ vs $f$ relationship in other types of network structure (e.g. Erdos-Renyi and Barabasi-Albert networks). One way of doing this is by introducing a parameter $\theta$ in the model. In this alternative model, $\overrightarrow{F_{a p p}}$ is allowed to have directions along $\pm \theta$ from the diagonal. For each edge, we choose an $\overrightarrow{F_{a p p}}$ direction within $[-\theta,-\theta+\Delta \theta,-\theta+2 \Delta \theta, \ldots, \theta]$. From here, one can then study both analytically and numerically the dependence of $f$ to $\theta$ which is a way of quantifying the degree of force direction randomness.

The application of our model to a real-world system was presented in this chapter. Future studies can investigate and verify the applicability of this framework to other kinds of real-world systems. Having a snapshot of a real-world network at different stages of its evolution can help in this endeavor of testing the dynamical model of directed network resilience under the presence of memory presented here. Additionally, most real-world systems are not only a singular network but a network of networks. Transportation networks, for example, are composed of interconnected networks representing the interaction of the different modes of transport. With the recent advancements in mathematically describing interconnected networks, a future direction of this work can be extending the current model to a system of network of networks. 


\section{Chapter 7}

\section{Conclusions and Future Work}

Human mobility shows complex patterns. It takes different scales and can be motivated by diverse reasons. In most indigenous human societies, it is the post-marital residence rule or kinship practice that governs movements of the populations. Such marriage motivated migrations have given rise to genetic variation pattern which cannot be explained using simple diffusion models. We present in Chapters 2-4, how kinship practices can be modelled to account for the existing genetic and linguistic diversities in the Indonesian islands of Sumba and Timor.

Languages have been found to be associated with genes even in the presence of sustained migration among communities. We show here that the extent of this association is a result of how structured dispersals are. Persistent adherence to kinship practices structures the movement of individuals among communities. This creates migration channels with sizes that are gender-dependent. Such system of dispersal in deep time shapes the genetics of the population. Through the same channels, language currents flow. Differences in language sharing between males and females can arise since transmission is restricted by kinship rules. Kinship is one of the many components of a culture's history that can explain the degree and persistence of both language sharing and genetic structure.

Furthermore, language trees have been observed to mirror gene trees from local to global scales. This suggests that languages and genes evolve in tandem as the communities that carry them split and diverge, but this model is inconsistent with the widespread mobility observed between communities. Instead we require a model that explains correlations between genes and languages, while accounting for high rates of migration and language adoption. Here, we show that the critical variable 
is that human movements are not random, but highly structured.

Languages are transmitted through channels created by kinship systems. Given sufficient time, these kinship channels can change the genetic and linguistic structure of populations. In traditional societies of eastern Indonesia, finely resolved co-phylogenies of languages and genes reveal persistent movements between stable speech communities facilitated by kinship rules. When multiple languages are present in a region and postmarital residence rules encourage sustained directional movement between speech communities, then languages should be channeled along uniparental lines. We find strong evidence for this pattern in 982 individuals from 25 villages on two adjacent islands, where different kinship rules have been followed. Core groups of close relatives stayed together for generations, while remaining in contact with, and marrying into, surrounding groups. Over time, kinship systems shaped their gene and language phylogenies: consistently following a postmarital residence rule turned social communities into speech communities.

In urban areas, human movements are studied in Chapter 5 as attractions between populations which depend both on the size of and distance between two populations. The propensity of an individual to choose to travel to one place over the other depends on the density and type of amenities available in the region and other regions competing for attraction.

Interactions and movements in urban systems are controlled by their transport networks. For these systems to function efficiently, there is a need to build a robust network. We present here how we can couple land use and network structure to model the travel demand of stations in a transport network. This gives us insights as to how transport networks should evolve with changing land use patterns. We apply the model to the Singapore Rapid Transit System (RTS). We find that considering the degree centrality of the network and using the gross plot ratio as the potential measure of how likely a commuter will be attracted to travel to specific areas, the predicted values of the model has a correlation of 0.70 with data. Considering morning and afternoon trips gives a slightly better correlation of 0.74 and 0.71 respectively. All predictions made by the proposed modified radiation model give the best correlation among all purely analytical models used to describe flow of commuters in a mass rapid transit system like the MRT.

Urban systems have the signature characteristic of intense interactions resulting 
from high-frequency movements of its inhabitants as enabled by an efficient and robust Rapid Transit System (RTS). While predictive models on human mobility are available at the scale of inter- and intra-country level, there is as yet few successful models that predict human movements at the urban scale. In this paper, we proposed a modified radiation model that is able to estimate with good accuracy the average mobility flux between the origin-destination stations of a real-world RTS. We achieved this by enhancing the original radiation model to incorporate information on land-use from the urban plan and coupling it with the RTS network structure. The inclusion of these details into the radiation model provides finer-scale information which accounts for the effect of spatial heterogeneity not possible in the original model. We apply the model to the Singapore Mass Rapid Transit (MRT) system by considering its degree centrality and using the gross plot ratio weighted by spatial entropy to determine how likely a commuter is attracted to travel to specific areas. Our results demonstrate improved prediction on urban travel demands compared to the gravity model and the original radiation model on real-world data.

In chapter 6 , we present a general framework for probing the dynamic evolution of spatial networks comprised of nodes applying force amongst each other. Aside from the already reported magnitude of forces and elongation thresholds, we show that preservation of links in a network is also crucially dependent on how nodes are connected and how edges are directed. We demonstrate that the time it takes for the networks to reach its equilibrium network structure follows a robust power law relationship consistent with Basquin's law with an exponent that can be tuned by changing only the force directions. Further, we illustrate that networks with different connection structures, node positions and edge directions have different Basquin's exponent which can be used to distinguish spatial directed networks from each other. Using an extensive waiting time simulation that spans up to over 16 orders of magnitude, we establish that the presence of memory combined with the scale-free bursty dynamics of edge breaking at the micro level leads to the evident macroscopic power law distribution of network lifetime.

Decreasing the magnitude of forces slows down fragmentation regardless of network structure, but is only successful in delaying the onset of fragmentation in lattice networks. For lattice networks, an increase in the randomization of force directions can further lead to a decrease in the rate of fragmentation. Changing the direction 
of forces has no effect in the rate of fragmentation for both ER and BA networks providing an indication as to why their final state were the same regardless of force directions.

The lifetimes of the networks defined as the amount of time it takes for the network to reach its equilibrium $\mathrm{f}$ are also found to have a power law behavior following Basquins Law. We further extend the work of Carmona et al. by demonstrating that Basquins exponent, $\gamma$, can be altered by changing only the force directions in the network without altering the network breaking thresholds. By looking at the waiting time between successive edge failures, we found that in the presence of deformation memory, the failure process proceeds in bursts with a universal scale-free distribution. This micro-scale bursty breaking of edges leads to the macro-scale power law distribution of network lifetime. This holds true for networks of very different degree distributions hinting to the universality of this mechanism.

There is no one way of modelling mobility. We find that one method that works for one type and scale of system does not work in other types and scales. There is a need to look at each system separately and construct a model that reflects the dynamics observed from the system. We see here that the ways of modelling mobility in rural and urban areas are in detail different. This results from differences in their histories and nature which give rise to different mechanisms. Furthermore, such systems are characterized by different spatial and temporal scales which makes the critical parameters controlling them different. In modelling population mobility in rural or traditional communities, the spatial and temporal scales are far higher. In such a system a more macroscopic model is necessary. Hence, the agent-based models, coalescent models, and co-phylogeny methods we employed looked at time in terms of generations and space in terms of villages. However, in the modelling of urban mobility, finer granularity of details was incorporated. Although predictions were still aggregated, the temporal scale we now considered are in the range of days to weeks. Such a temporal scale warrants looking at routines and activity-based movements. This then means that we also need to zoom into the details of space. Hence, we looked at amenities, land-use, and actual interparticle forces.

A possible marriage between these two different modelling paradigm is looking at temporal and spatial scales that are between them, that is a temporal scale of years and an inter-city spatial scale. Is it possible then to build a unifying mobility 
framework that works in this scale as well as in the urban scale. Similar to what the gravity and original radiation models first envisioned but failed to achieve, can we develop a mobility model based on first principles that performs well in inter-country, inter-city and intra-city travels? 


\section{Bibliography}

[1] R. Albert, H. Jeong, and A. L. Barabasi. Error and attack tolerance of complex networks. Nature, 406(378-382), 2000.

[2] Kyle F Davis, Paolo D Odorico, Francesco Laio, and Luca Ridolfi. Global Spatio-Temporal Patterns in Human Migration : A Complex Network Perspective. Plos One, 8(1), 2013.

[3] J.F.C. Kingman and Conference Board of the Mathematical Sciences. Mathematics of Genetic Diversity. CBMS-NSF Regional Conference Series in Applied Mathematics. Society for Industrial and Applied Mathematics, 1980.

[4] J Stephen Lansing, Murray P Cox, Sean S Downey, Brandon M Gabler, Brian Hallmark, Tatiana M Karafet, Peter Norquest, John W Schoenfelder, Herawati Sudoyo, Joseph C Watkins, et al. Coevolution of languages and genes on the island of sumba, eastern indonesia. Proceedings of the National Academy of Sciences, 104(41):16022-16026, 2007.

[5] Meryanne K Tumonggor, Tatiana M Karafet, Brian Hallmark, J Stephen Lansing, Herawati Sudoyo, Michael F Hammer, and Murray P Cox. The indonesian archipelago: an ancient genetic highway linking asia and the pacific. Journal of human genetics, 58(3):165-173, 2013.

[6] Erika Fille Legara, Christopher Monterola, Kee Khoon Lee, and Gih Guang Hung. Critical capacity, travel time delays and travel time distribution of rapid mass transit systems. Physica A: Statistical Mechanics and its Applications, 406:100-106, 2014. 
[7] Filippo Simini, Marta C González, Amos Maritan, and Albert-László Barabási. A universal model for mobility and migration patterns. Nature, 484(7392):96-100, 2012.

[8] Marta C Gonzalez, Cesar A Hidalgo, and Albert-Laszlo Barabasi. Understanding individual human mobility patterns. Nature, 453(7196):779-782, 2008.

[9] I Farkas, I Derényi, H Jeong, Z Neda, ZN Oltvai, E Ravasz, A Schubert, A-L Barabási, and T Vicsek. Networks in life: Scaling properties and eigenvalue spectra. Physica A: Statistical Mechanics and its Applications, 314(1):25-34, 2002.

[10] Petter Holme, Christofer R Edling, and Fredrik Liljeros. Structure and time evolution of an internet dating community. Social Networks, 26(2):155-174, 2004 .

[11] Cesar A Hidalgo and C Rodriguez-Sickert. The dynamics of a mobile phone network. Physica A: Statistical Mechanics and its Applications, 387(12):30173024, 2008.

[12] Ronald S Burt. Decay functions. Social networks, 22(1):1-28, 2000.

[13] Gergely Palla, Albert-László Barabási, and Tamás Vicsek. Quantifying social group evolution. Nature, 446(7136):664-667, 2007.

[14] Gueorgi Kossinets and Duncan J Watts. Empirical analysis of an evolving social network. Science, 311(5757):88-90, 2006.

[15] Cheryl Abundo, Christopher Monterola, and Erika Fille Legara. Criticality of forcing directions on the fragmentation and resilience of grid networks. Nature Scientific Reports, pages 1-9, 2014.

[16] Tatiana M Karafet, Brian Hallmark, Murray P Cox, Herawati Sudoyo, Sean Downey, J Stephen Lansing, and Michael F Hammer. Major east-west division underlies y chromosome stratification across indonesia. Molecular biology and evolution, 27(8):1833-1844, 2010.

[17] Meryanne K Tumonggor, Tatiana M Karafet, Sean Downey, J Stephen Lansing, Peter Norquest, Herawati Sudoyo, Michael F Hammer, and Murray P 
Cox. Isolation, contact and social behavior shaped genetic diversity in West Timor. Journal of human genetics, 59(9):494-503, 2014.

[18] Elsa G Guillot, Martin L Hazelton, Tatiana M Karafet, J Stephen Lansing, Herawati Sudoyo, and Murray P Cox. Relaxed observance of traditional marriage rules allows social connectivity without loss of genetic diversity. Molecular biology and evolution, page msv102, 2015.

[19] Petter Holme and Jari Saramäki. Temporal networks. Physics reports, 519(3):97-125, 2012.

[20] G V Dziebel. The Genius of Kinship: The Phenomenon of Human Kinship and the Global Diversity of Kinship Terminologies. Cambria Press, 2007.

[21] Isabel Alves, Anna Šrámková Hanulová, Matthieu Foll, and Laurent Excoffier. Genomic data reveal a complex making of humans. PLoS Genetics, 8(7), 2012.

[22] George Peter Murdock. Social structure. Macmillan, 1949.

[23] Fiona M Jordan, Russell D Gray, Simon J Greenhill, and Ruth Mace. Matrilocal residence is ancestral in austronesian societies. Proceedings of the Royal Society of London B: Biological Sciences, pages rspb-2009, 2009.

[24] Mark R Rosenzweig and Oded Stark. Consumption smoothing, migration, and marriage: Evidence from rural india. The Journal of Political Economy, pages 905-926, 1989.

[25] Jack Goody. Production and reproduction: a comparative study of the domestic domains. Cambridge University Press, 1976.

[26] Michael Krawczak and Robert H Barnes. How obedience of marriage rules may counteract genetic drift. Journal of community genetics, 1(1):23-28, 2010.

[27] M van Oven and M Kayser. Updated comprehensive phylogenetic tree of global human mitochondrial dna variation. Human Mutation, 30(2):E386E394, 2009.

[28] International Society of Genetic Genealogy. International society of genetic genealogy, 2017. 
[29] Tatiana M Karafet, Fernando L Mendez, Monica B Meilerman, Peter A Underhill, Stephen L Zegura, and Michael F Hammer. New binary polymorphisms reshape and increase resolution of the human Y chromosomal haplogroup tree. Genome Research, pages 830-838, 2008.

[30] Ruzica Bruvo, Nicolaas K. Michiels, Thomas G. D'souza, and Hinrich Schulenburg. A simple method for the calculation of microsatellite genotype distances irrespective of ploidy level. Molecular Ecology, 13(7):2101-2106, 2004.

[31] Qiaomei Fu, Alissa Mittnik, Philip L. F. Johnson, Kirsten Bos, Martina Lari, Ruth Bollongino, Chengkai Sun, Liane Giemsch, Ralf Schmitz, Joachim Burger, Anna Maria Ronchitelli, Fabio Martini, Renata G. Cremonesi, Jiř́ Svoboda, Peter Bauer, David Caramelli, Sergi Castellano, David Reich, Svante Pääbo, and Johannes Krause. A revised timescale for human evolution based on ancient mitochondrial genomes. Current Biology, 23(7):553-559, 2017/07/11 2013.

[32] E. Paradis. Analysis of Phylogenetics and Evolution with R. Use R! Springer New York, 2006.

[33] Emmanuel Paradis, Julien Claude, and Korbinian Strimmer. Ape: Analyses of phylogenetics and evolution in r language. Bioinformatics, 20(2):289, 2004.

[34] J F C Kingman. On the Genealogy of Large Populations. Journal of Applied Probability, 19:27-43, 1982.

[35] R. a. Blythe and a. J. McKane. Stochastic models of evolution in genetics, ecology and linguistics. Journal of Statistical Mechanics: Theory and Experiment, 2007(07):P07018-P07018, 2007.

[36] J. Stephen Lansing, Murray P. Cox, Therese A. de Vet, Sean S. Downey, Brian Hallmark, and Herawati Sudoyo. An ongoing austronesian expansion in island southeast asia. Journal of Anthropological Archaeology, 30(3):262-272, 92011.

[37] Edward Sapir. Language: An Introduction to the Study of Speech. Harcourt, Brace \& Co.: New York, 1949. 
[38] W Tecumseh Fitch. Kin selection and 'mother tongues': a neglected component in language evolution. Evolution of communication systems: A comparative approach, pages 275-296, 2004.

[39] Arturo Tosi. The notion of "community" in language maintenance. Bilingualism and migration, pages 325-343, 1999.

[40] Alan Barnard. The co-evolution of language and kinship. Early human kinship: from sex to social reproduction, pages 232-243, 2008.

[41] JS Lansing, MP Cox, SS Downey, BM Gabler, B Hallmark, TM Karafet, P Norquest, JW Schoenfelder, H Sudoyo, JC Watkins, and MF Hammer. Coevolution of languages and genes on the island of Sumba, eastern Indonesia. Proceedings of the National Academy of Sciences USA, 104:16022-16026, 2007.

[42] JS Lansing, MP Cox, TA de Vet, SS Downey, B Hallmark, and H Sudoyo. An ongoing Austronesian expansion in Island Southeast Asia. Journal of Anthropological Archaeology, 30:262-272, 2011.

[43] EG Guillot, ML Hazelton, TM Karafet, JS Lansing, H Sudoyo, and MP Cox. Relaxed observance of traditional marriage rules allows social connectivity without loss of genetic diversity. Molecular Biology and Evolution, 32:22542262, 2015.

[44] Patricia Balaresque and Mark A Jobling. Human populations: houses for spouses. Current Biology, 17(1):R14-R16, 2007.

[45] George Peter Murdock. Social Structure. Macmillan: London, 1949.

[46] Alan Barnard. Kinship, language and production: a conjectural history of khoisan social structure. Africa, pages 29-50, 1988.

[47] L Luca Cavalli-Sforza. Genes, peoples, and languages. Proceedings of the National Academy of Sciences, 94(15):7719-7724, 1997.

[48] Oleg Balanovsky, Khadizhat Dibirova, Anna Dybo, Oleg Mudrak, Svetlana Frolova, Elvira Pocheshkhova, Marc Haber, Daniel Platt, Theodore Schurr, Wolfgang Haak, et al. Parallel evolution of genes and languages in the caucasus region. Molecular biology and evolution, 28(10):2905-2920, 2011. 
[49] Evelyne Heyer, Patricia Balaresque, Mark A Jobling, Lluis Quintana-Murci, Raphaelle Chaix, Laure Segurel, Almaz Aldashev, and Tanya Hegay. Genetic diversity and the emergence of ethnic groups in central asia. Bmc Genetics, 10(1):49, 2009.

[50] Sibylle M Gomes, Mannis van Oven, Luis Souto, Helena Morreira, Silke Brauer, Martin Bodner, Bettina Zimmermann, Gabriela Huber, Christina Strobl, Alexander W Röck, et al. Lack of gene-language correlation due to reciprocal female but directional male admixture in Austronesians and nonAustronesians of East Timor. European Journal of Human Genetics, 25:246$252,2017$.

[51] Jill Forshee. Review: Wehali - The Female Land: Traditions of a Timorese Ritual Centre, by Tom Therik. Indonesia, 82:133-137, 2006.

[52] Hilde M Wilkinson-Herbots. The distribution of the coalescence time and the number of pairwise nucleotide differences in the "Isolation with Migration" model. Theoretical Population Biology, 73:277-288, 2008.

[53] Hilde M Wilkinson-Herbots. A fast method to estimate speciation parameters in a model of isolation with an initial period of gene flow and to test alternative evolutionary scenarios. arXiv, pages 1-16, 2015.

[54] Hilde M. Wilkinson-Herbots. The distribution of the coalescence time and the number of pairwise nucleotide differences in a model of population divergence or speciation with an initial period of gene flow. Theoretical Population Biology, 82(2):92-108, 2012.

[55] Rui J. Costa and Hilde Wilkinson-Herbots. The generalised isolation-withmigration model: a maximum-likelihood implementation for multilocus data sets. arXiv, pages 1-14, 2016.

[56] Rui J. Costa and Hilde Wilkinson-Herbots. Efficient maximum-likelihood inference for the isolation-with-initial-migration model with potentially asymmetric gene flow. arXiv, pages 1-33, 2016. 
[57] Pierre Legendre, Yves Desdevises, Eric Bazin, and Roderic D. M. Page. A statistical test for host-parasite coevolution. Systematic Biology, 51(2):217, 2002.

[58] Stéphane Dray and Pierre Legendre. Testing the species traits-environment relationships: The fourth-corner problem revisited. Ecology, 89(12):3400-3412, 2008.

[59] Jamshid J Tehrani, Mark Collard, and Stephen J Shennan. The cophylogeny of populations and cultures: reconstructing the evolution of iranian tribal craft traditions using trees and jungles. Philosophical Transactions of the Royal Society B: Biological Sciences, 365(1559):3865-3874, 2010.

[60] Keith Hunley. Reassessment of global gene-language coevolution. Proceedings of the National Academy of Sciences of the United States of America, 112(7):1919-1920, 022015.

[61] Nicole Creanza, Merritt Ruhlen, Trevor J Pemberton, Noah A Rosenberg, Marcus W Feldman, and Sohini Ramachandran. A comparison of worldwide phonemic and genetic variation in human populations. Proceedings of the National Academy of Sciences of the United States of America, 112(5):12651272, 022015.

[62] T Therik. Wehali - The Female Land: Traditions of a Timorese Ritual Centre. Pandanus Books: ANU Research School of Pacific and Asian Studies, Canberra, Australia, 2004.

[63] F Vallée, A Luciani, and MP Cox. Reconstructing demography and social behavior during the Neolithic expansion from genomic diversity across Island Southeast Asia. Genetics, 204:1495-1506, 2016.

[64] Nicole Creanza, Merritt Ruhlen, Trevor J Pemberton, Noah A Rosenberg, Marcus W Feldman, and Sohini Ramachandran. A comparison of worldwide phonemic and genetic variation in human populations. Proceedings of the National Academy of Sciences USA, 112:1265-1272, 2015.

[65] Ernst Georg Ravenstein. The laws of migration. Journal of the Statistical Society of London, 48(2), 1885. 
[66] Samuel A Stouffer. Intervening opportunities: A theory relating mobility and distance. American Sociological Review, 5(6):845-867, 1940.

[67] George Kingsley Zipf. The p1 p2 / d hypothesis : On the intercity movement of persons. American Sociological Review, 11(6):677-686, 1946.

[68] Xiao Liang, Jichang Zhao, Li Dong, and Ke Xu. Unraveling the origin of exponential law in intra-urban human mobility. 2013.

[69] Xiao-yong Yan, Chen Zhao, Ying Fan, Zengru Di, and Wen-xu Wang. Universal predictability of mobility patterns in cities. pages $1-18$.

[70] Gallotti Riccardo, Bazzani Armando, and Rambaldi Sandro. Towards a statistical physics of human mobility. International Journal of Modern Physics C, 23(9), 2012.

[71] Minjin Lee and Petter Holme. Relating land use and human intra-city mobility. Plos One, pages 1-14, 2015.

[72] Alexey Tarasov, Felix Kling, and Alexei Pozdnoukhov. Prediction of user location using the radiation model and categories and subject descriptors. UrbComp'13, 2013.

[73] Segun Goh, Keumsook Lee, Jong Soo Park, and M Y Choi. Modification of the gravity model and application to the metropolitan Seoul subway system. Physical Review E, 86(026102):1-6, 2012.

[74] Inho Hong and Woo-sung Jung. Application of gravity model on the Korean urban bus network. Physica A, 462:48-55, 2016.

[75] T Alex Perkins, Andres J Garcia, Valerie A Paz-solda, Steven T Stoddard, Robert C Reiner Jr, Gonzalo Vazquez-prokopec, Donal Bisanzio, Amy C Morrison, Eric S Halsey, Tadeusz J Kochel, David L Smith, Uriel Kitron, Thomas W Scott, and Andrew J Tatem. Theory and data for simulating fine-scale human movement in an urban environment. Journal of The Royal Society Interface, 2014. 
[76] A Paolo Masucci, Joan Serras, Anders Johansson, and Michael Batty. Gravity versus radiation models : On the importance of scale and heterogeneity in commuting flows. Physical Review E, 88(022812):1-8, 2013.

[77] Filippo Simini and Amos Maritan. Human Mobility in a Continuum Approach. 8(3), 2013.

[78] Yingxiang Yang, Carlos Herrera, Nathan Eagle, and Marta C Gonza. Limits of Predictability in Commuting Flows in the Absence of Data for. Nature Scientific Reports, 4(5662):1-9, 2014.

[79] Sebastian Grauwin, Michael Szell, Stanislav Sobolevsky, Philipp Hövel, Filippo Simini, Maarten Vanhoof, Zbigniew Smoreda, and Albert-lászló Barabási. Identifying and modeling the structural discontinuities of human interactions. Nature Scientific Reports, 7(46677):1-11, 2017.

[80] Yihui Ren, Maria Ercsey-Ravasz, Pu Wang, Marta C Gonzalez, and Zoltan Toroczkai. Predicting commuter flows in spatial networks using a radiation model based on temporal ranges. Nature Communications, 5(5347), 2014.

[81] Xiao-yong Yan, Chen Zhao, Ying Fan, Zengru Di, Wen-xu Wang, and Wen-xu Wang. Universal predictability of mobility patterns in cities. Journal of The Royal Society Interface, 11(20140834), 2014.

[82] Liang Xiao, Zhao Jichang, and X U Ke. A general law of human mobility. Science China: Information Sciences, 58(100103):1-14, 2015.

[83] Chaogui Kang, Yu Liu, Diansheng Guo, and Kun Qin. A Generalized Radiation Model for Human Mobility: Spatial Scale, Searching Direction and Trip Constraint. Plos One, 10(11):1-11, 2015.

[84] Maxime Lenormand, Aleix Bassolas, and José J Ramasco. Systematic comparison of trip distribution laws and models. JTRG, 51:158-169, 2016.

[85] H. C. Carey. Principles of Social Science. The University of Michigan, 1859.

[86] Morton Schneider. Gravity models and trip distribution theory. Papers in Regional Science, 5(1):51-56, 1959. 
[87] Urban Redevelopment Authority. URA Master Plan 2008 retrieved March 2013 from www.ura.gov.sg/uramaps/.

[88] James Decraene, Christopher Monterola, Gary Kee, Khoon Lee, Terence Gih, and Guang Hung. A Quantitative Procedure for the Spatial Characterization of Urban Land Use. International Journal of Modern Physics C, 24(1):1-15, 2013.

[89] James Decraene, Christopher Monterola, Gary Kee, Khoon Lee, Terence Gih, Guang Hung, and Michael Batty. The Emergence of Urban Land Use Patterns Driven by Dispersion and Aggregation Mechanisms. Plos One, 8(12), 2013.

[90] David Levinson and Ahmed El-geneidy. Regional Science and Urban Economics The minimum circuity frontier and the journey to work. Regional Science and Urban Economics, 39(6):732-738, 2009.

[91] David Levinson. Network Structure and City Size. Plos One, 7(1), 2012.

[92] Nasri Bin Othman, Erika Fille Legara, Vicknesh Selvam, and Christopher Monterola. Simulating Congestion Dynamics of Train Rapid Transit using Smart Card Data. Procedia - Procedia Computer Science, 29:1610-1620, 2014.

[93] Lijun Sun, Der-horng Lee, Alex Erath, and Xianfeng Huang. Using Smart Card Data to Extract Passenger's Spatio-temporal Density and Train's Trajectory of MRT System. ACM UrbComp, 2012.

[94] Chen Zhong, Stefan Müller Arisona, Xianfeng Huang, Michael Batty, Gerhard Schmitt, Chen Zhong, Stefan Müller Arisona, Xianfeng Huang, and Michael Batty. Detecting the dynamics of urban structure through spatial network analysis. International Journal of Geographical Information Science, 28(11):2178-2199, 2014.

[95] Chen Zhong, Ed Manley, Stefan Müller, Michael Batty, and Gerhard Schmitt. Measuring variability of mobility patterns from multiday smart-card data. Journal of Computational Science, 9:125-130, 2015.

[96] Shan Jiang, Joseph Ferreira, and Marta C Gonzalez. Activity-Based Human Mobility Patterns Inferred from Mobile Phone Data : A Case Study of Singapore. IEEE Transactions on Big Data, 3(2):208-219, 2017. 
[97] Anastasios Noulas, Salvatore Scellato, Renaud Lambiotte, Massimiliano Pontil, and Cecilia Mascolo. A Tale of Many Cities: Universal Patterns in Human Urban Mobility. Plos One, 7(5), 2012.

[98] Markus Schläpfer, Luís M A Bettencourt, Sébastian Grauwin, Mathias Raschke, Rob Claxton, Zbigniew Smoreda, Geoffrey B West, Carlo Ratti, M A Bettencourt, Markus Schla, Mathias Raschke, Rob Claxton, Zbigniew Smoreda, Geoffrey B West, and Carlo Ratti. The scaling of human interactions with city size The scaling of human interactions with city size. Journal of The Royal Society Interface, 11(20130789), 2014.

[99] Jameson L Toole, Carlos Herrera-yaqu, Christian M Schneider, and Marta C Gonzalez, Marta C. Coupling human mobility and social ties. Journal of The Royal Society Interface, 12(20141128), 2015.

[100] M. Gastner and M. Newman. The spatial structure of networks. Eur. Phys. J. B, 49:247-252, 2006.

[101] D. Yamins and S. Rasmussen. Growing urban roads. Networks and Spatial Economics, 2003.

[102] P. Ringler and D. Schulz. Self-assembly of proteins into designed networks. Science, 302:106-109, 2003.

[103] G. Barrenetxea, B. Beferull-Lozano, and M. Vetterli. Lattice networks: capacity limits, optimal routing, and queueing behavior. IEEE/ACM Trans. Netw., 14:492-505, 2006.

[104] Petter Holme, Beom Jun Kim, Chang No Yoon, and Seung Kee Han. Attack vulnerability of complex networks. 65:1-14, 2002.

[105] Jianxi Gao, Xueming Liu, Daqing Li, and Shlomo Havlin. Recent Progress on the Resilience of Complex Networks. Energies, 8:12187-12210, 2015.

[106] Albert-László Barabási. Network science. Cambridge university press, 2016.

[107] Réka Albert and Albert-László Barabási. Statistical mechanics of complex networks. Rev. Mod. Phys., 74:47-97, Jan 2002. 
[108] R. Cohen, K. Erez, D. ben Avraham, and S. Havlin. Breakdown of the internet under intentional attacks. Physical Review Letters, 86:3682-3685, 2001.

[109] D. Callaway, M. Newman, S. Strogatz, and D. Watts. Network robustness and fragility: percolation on random graphs. Physical Review Letters, 85:5468$5471,2000$.

[110] Daqing Li, Bowen Fu, Yunpeng Wang, Guangquan Lu, Yehiel Berezin, H. Eugene Stanley, and Shlomo Havlin. Percolation transition in dynamical traffic network with evolving critical bottlenecks. Proceedings of the National Academy of Sciences, 112(3):669-672, 2015.

[111] Eran Segal, Michael Shapira, Aviv Regev, Dana Pe'er, David Botstein, Daphne Koller, and Nir Friedman. Module networks: identifying regulatory modules and their condition-specific regulators from gene expression data. Nat Genet, 34(2):166-176, 062003.

[112] Gouhei Tanaka, Kai Morino, and Kazuyuki Aihara. Dynamical robustness in complex networks: the crucial role of low-degree nodes. Scientific Reports, $2: 232,2012$.

[113] Duncan J. Watts. A simple model of global cascades on random networks. Proceedings of the National Academy of Sciences, 99(9):5766-5771, 2002.

[114] Francesca Benuzzi, Giovanna Zamboni, Stefano Meletti, Marco Serafini, Fausta Lui, Patrizia Baraldi, Davide Duzzi, Guido Rubboli, Carlo Alberto Tassinari, and Paolo Frigio Nichelli. Recovery from emotion recognition impairment after temporal lobectomy. Frontiers in Neurology, 5:92, 2014.

[115] Antonio Majdandzic, Boris Podobnik, Sergey V. Buldyrev, Dror Y. Kenett, Shlomo Havlin, and H. Eugene Stanley. Spontaneous recovery in dynamical networks. Nat Phys, 10(1):34-38, 012014.

[116] C. De Marco. A phase transition model for cascading network failure. Control Sys. Mag., 21:40-51, 2001.

[117] J. Wang and L. Rong. Edge-based-attack induced cascading failures on scalefree networks. Physica A: Statistical Mechanics and its Applications, 388:17311737,2009 
[118] D. Nedic, I. Dobson, D. Kirschen, B. Carreras, and V. Lynch. Criticality in a cascading failure blackout model. Int. J. of Electrical Power and Energy Sys, 28:627-633, 2006.

[119] H. A. Carmona, F. Kun, J. S. Andrade Jr., and H. J. Herrmann. Computer simulation of fatigue under diametrical compression. Physical Review E, 75:046115, 2007.

[120] G. A. Esleta and C. Monterola. Structural reinforcement in a spring-block model of stress-induced fracture propagation. Computer Physics Communications, 178:625-646, 2008.

[121] G. A. Esleta and C. Monterola. Effect of memory and reinforcement on the propagation and morphology of fracture in a two-dimensional mass-spring system. International Journal of Modern Physics C, 20:1049-1062, 2009.

[122] R. Albert and A. L. Barabasi. Statistical mechanics of complex networks. Rev. Mod. Phys., 74:47-97, 2002.

[123] A. L. Barabasi. Scale-free networks: a decade and beyond. Science, 325:412413, 2009.

[124] A. L. Barabasi, H. Jeong, Z. Neda, E. Ravasz, A. Schubert, and T. Vicsek. Evolution of the social network of scientific collaborations. Physica A: Statistical Mechanics and its Applications, 311:590-614, 2002.

[125] A. L. Barabasi, R. Albert, and H. Jeong. Scale-free characteristics of random networks: the topology of the world-wide web. Physica A: Statistical Mechanics and its Applications, 281:69-77, 2000.

[126] Jin-hua Zhao. Generalized k-core pruning process on directed networks. arXiv, 2017.

[127] F. Kun, H. A. Carmona, and H. J. Herrmann. Universality behind basquin's law of fatigue. Physical Review Letters, 100:094301, 2008.

[128] Duncan S Callaway, Mark EJ Newman, Steven H Strogatz, and Duncan J Watts. Network robustness and fragility: Percolation on random graphs. Physical review letters, 85(25):5468, 2000. 
[129] Michael T Gastner and Mark EJ Newman. The spatial structure of networks. The European Physical Journal B-Condensed Matter and Complex Systems, 49(2):247-252, 2006.

[130] Daniel Yamins, Steen Rasmussen, and David Fogel. Growing urban roads. Networks and Spatial Economics, 3(1):69-85, 2003.

[131] Jian-Wei Wang and Li-Li Rong. Edge-based-attack induced cascading failures on scale-free networks. Physica A: Statistical Mechanics and its Applications, 388(8):1731-1737, 2009.

[132] C. Monterola. Cascade of travel duration delays in a rapid transport system. In European Conference on Complex Systems 2013, 2013. 


\section{Appendix A}

\section{Appendix}

\section{A.1 Network Elongation}

Initially, each edge is assigned an elongation threshold $\varepsilon_{t h}$. Before the crack propagation begins, the edges are structurally reinforced randomly such that the elongation thresholds of the edges are between 0.0500 and 0.0550 , similar to the method employed in fracture propagation [120]. Every two connected nodes differ in the amount of force loading they can manage to have between them. Randomly assigning elongation threshold values takes into account this disparity.
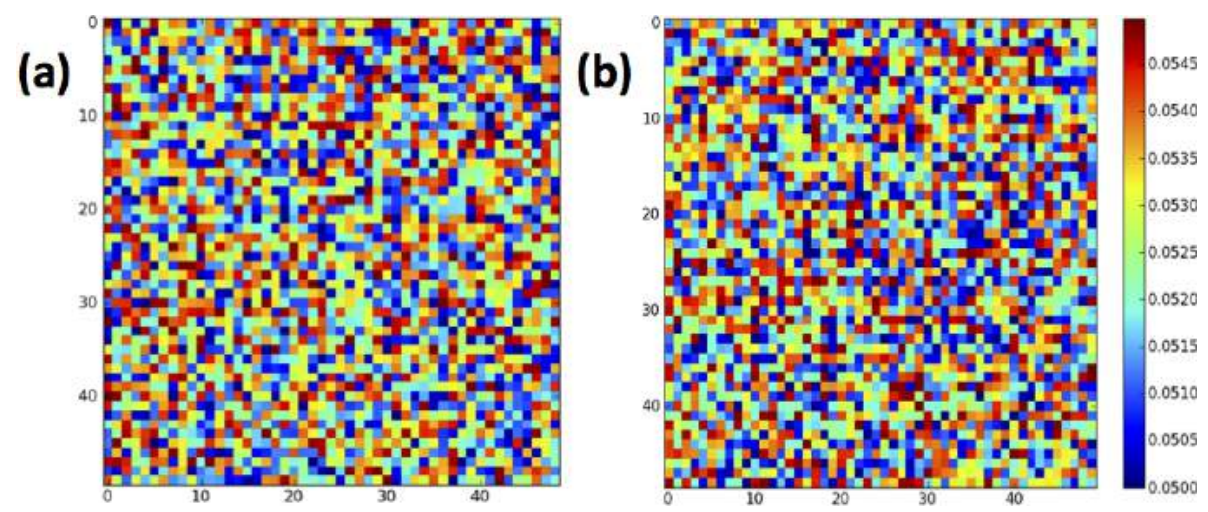

Figure A.1: Random reinforcement of network edges. (a) Elongation thresholds of the horizontal edges and (b) elongation thresholds of the vertical edges in the lattice network. 


\section{A.2 Numerical Stability}

The $2 N^{2}$ equations for node positions and $2 N^{2}$ equations for velocities were solved using the fourth order Runge-Kutta (RK4) method with known local discretisation error $O\left(\delta t^{5}\right)$ per time step iteration [120]. For $\delta t=2^{-10}$, the local error $\delta \xi_{L}$ is negligible at $9 x 10^{-16}$ per RK4 application. The global error $\delta \xi$, on the other hand, is $O\left(\delta t^{4}\right)$ and for our simulations involving an $N x N$ grid network described by $4 N^{2}$ differential equations, the total accumulated error for the entire network lifetime $t_{f}$ is $\delta \xi \sim 4 N^{2} t_{f}(\delta t)^{4}[120]$. With $\delta t=2^{-10}, N=50$ and $t_{f} \sim O\left(10^{5}\right), \delta \xi \sim 9 x 10^{-4}$ giving an error that is less than $0.1 \%$ for both node position and velocity. These values give no significant position error and time delay on the onset of fragmentation [120].

By subjecting a two-dimensional lattice to biaxial stretching and looking at the conservation of energy injected in the system, Esleta et al. also found numerical stability of the method with respect to lattice size . From their study, the waiting time $t_{c}$ which indicates the onset of breaking was found to be linear with respect to lattice size $N$ [120]. $t_{c} \sim N$ and the average force in each node $\left\langle F_{\text {node }}\right\rangle \sim$ $4 N F_{a p p}$ which means that the Hamiltonian will have constant increase with larger grid network sizes. This justifies the choice of our parameters as they are shown to still capture the dynamics of larger grid networks while reducing computational complexity (for RK4, computational complexity is proportional to $N^{2}$ ) [120, 121].

\section{A.3 Distribution of Influence}

\section{A.3.1 Lattice Network}

\section{A.3.1.1 Uniform Force Directions}

Forces in a system can be directed uniformly such that the vertical and horizontal applied forces exerted by connected nodes have the same directions. This results to a net force directed diagonally in the network as illustrated in Fig. A.2. These initial directions of $F_{a p p}^{k}$ between neighboring nodes are maintained throughout the simulation.

Here, the node in the topmost left corner labelled as the source node in Fig. A.2 (a) is the least influential node and is influenced by all nodes in the network. Such 


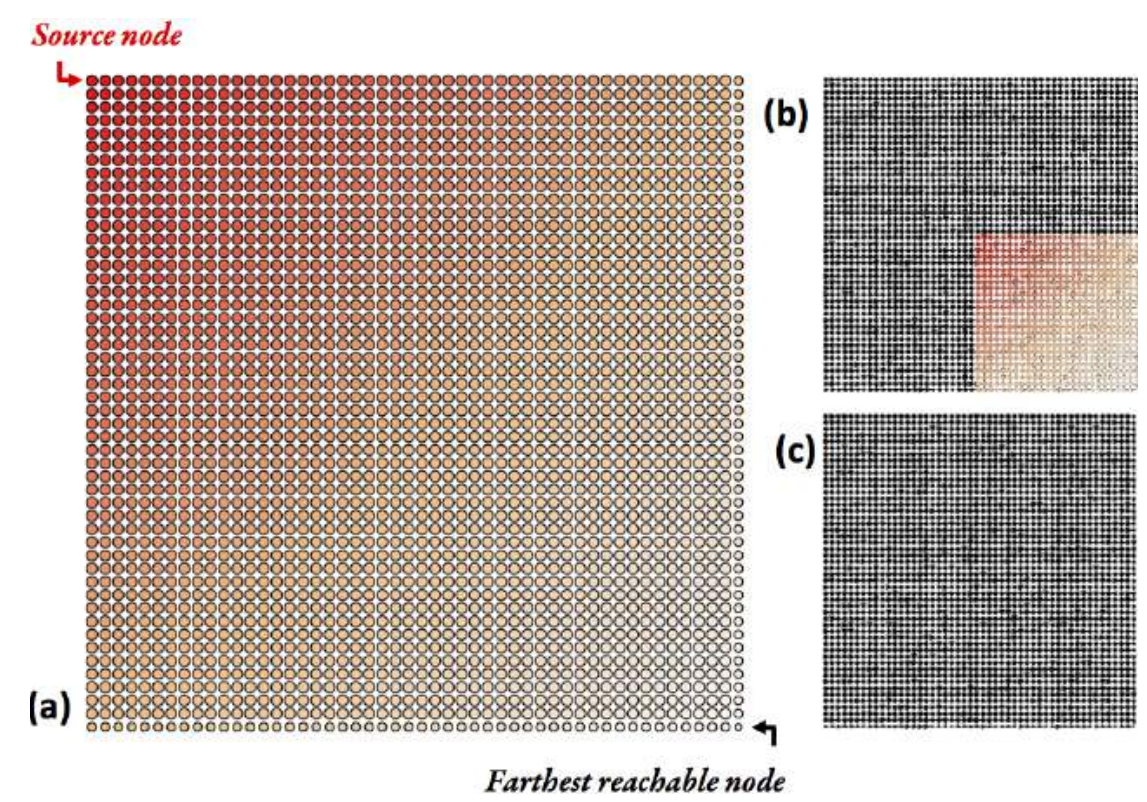

Figure A.2: Heatmap of a lattice network with uniform force directions. (a) The node in the topmost left corner is influenced by all other nodes and the degree of influence decreases diagonally towards the farthest reachable node at the bottom right corner. (b) Nodes are influenced only by nodes that are to their right and below them. (c) All forces are directed towards the most influential node in the bottom right corner.

force directions pattern also allows nodes to be influenced only by nodes that are to their right and below them as illustrated in Fig. A.2 (b). All forces in the system flow towards the node in the bottom right corner which is the most influential node in the network. This is also the only node which is not influenced by any node in the network.

\section{A.3.1.2 Random Force Directions}

The directions of the applied forces in a lattice network are random when the interactions of its components result to random distribution of loading and when the influential nodes of the network are positioned randomly in grid space. We show in Fig. A.3 that some nodes are influenced by only a few nodes while others receive much influence. When the force directions are random and not uniform, there are also more agents $(6.24 \%)$ which are not influenced by any node in the network. 


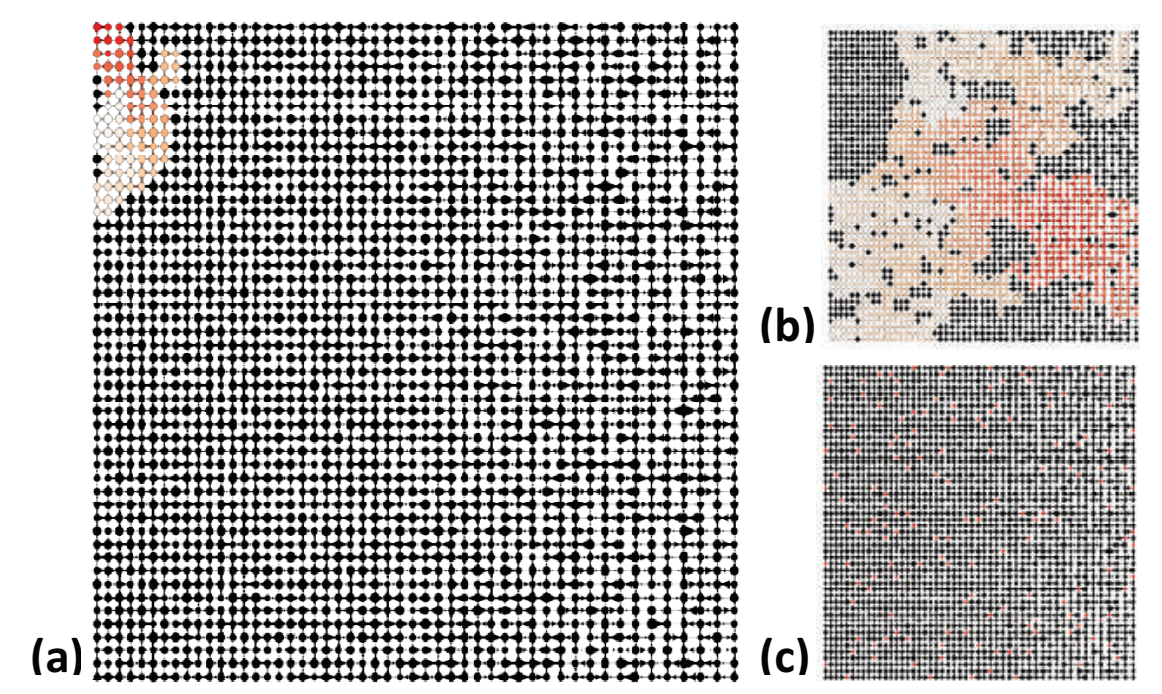

Figure A.3: Heatmap of a lattice network with random force directions. Influence is spatially distributed randomly. In (a) only $3.92 \%$ of the nodes has influence on the node in the topmost left corner of the network but in (b) we show a node having a greater influence range. (c) There are also more nodes $(6.24 \%)$ which are not influenced by any node.

\section{A.3.2 Erdos-Renyi Network}

\section{A.3.2.1 Uniform Force Directions}

Though connections among nodes in an Erdos-Renyi network are random, force directions can be made as uniform as possible by preferring specific positions in placing influential nodes. In Fig. A.4, we see that nodes are influenced only by nodes that are below them. The number of nodes that are not influence by any node accounts for $6.24 \%$ of nodes in the network which is similar to the case of lattice networks with random force directions.

\section{A.3.2.2 Random Force Directions}

In randomizing force directions in an Erdos-Renyi network, we are also distributing the influentials in grid space. For such case, nodes are influenced by other nodes regardless of their position as shown in Fig. A.5. There are also more nodes which aren't influenced by any node and this accounts for $14.12 \%$ of nodes in the network. 


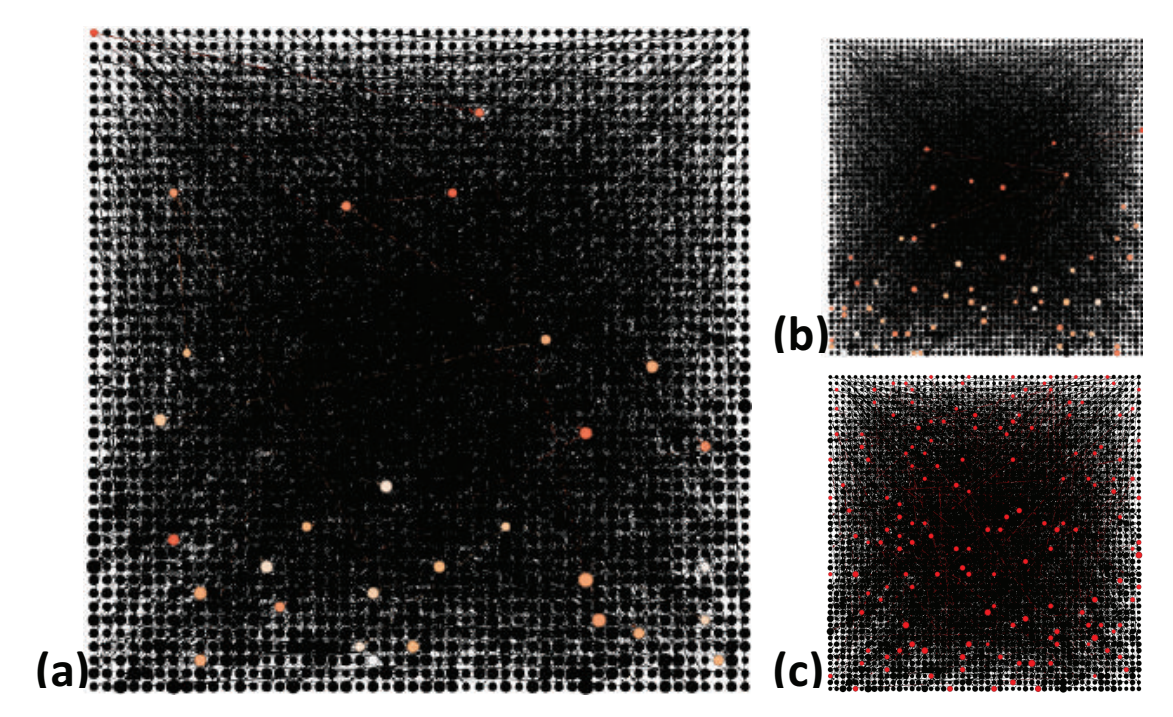

Figure A.4: Heatmap of an Erdos-Renyi network with uniform force directions. (a) Nodes can influence nodes that are not their spatial neighbors. The uniformity of force directions is illustrated in (b) where nodes can only be influenced by nodes below them. (c) The number of nodes that are not influenced by any node accounts for $6.24 \%$ of nodes in the network.

\section{A.3.3 Barabasi-Albert Network}

\section{A.3.3.1 Uniform Force Directions}

Almost all of the edges of the Barabasi-Albert network considered were directed outward and all non-zero node in-degrees are only at most $k_{i n}=2$. Owing to the scale-free degree distribution of the Barabasi-Albert network, the hubs account for almost all of the edges. By restricting all of the edges of the hubs to be directed outward, and all others to prefer a certain direction, uniformity in force directions can be attained as shown in Fig. A.6. This resulted to the hubs having a path to almost all nodes while other nodes have very few connections. $49.28 \%$ of the nodes has no outgoing edge and hence resulted to most of the nodes having only a few path connections.

\section{A.3.3.2 Random Force Directions}

For the Barabasi-Albert network with random force directions, the number of edges directed outward is about the same as those directed inward. Randomizing force directions created more paths among the nodes. All nodes regardless of their degree 

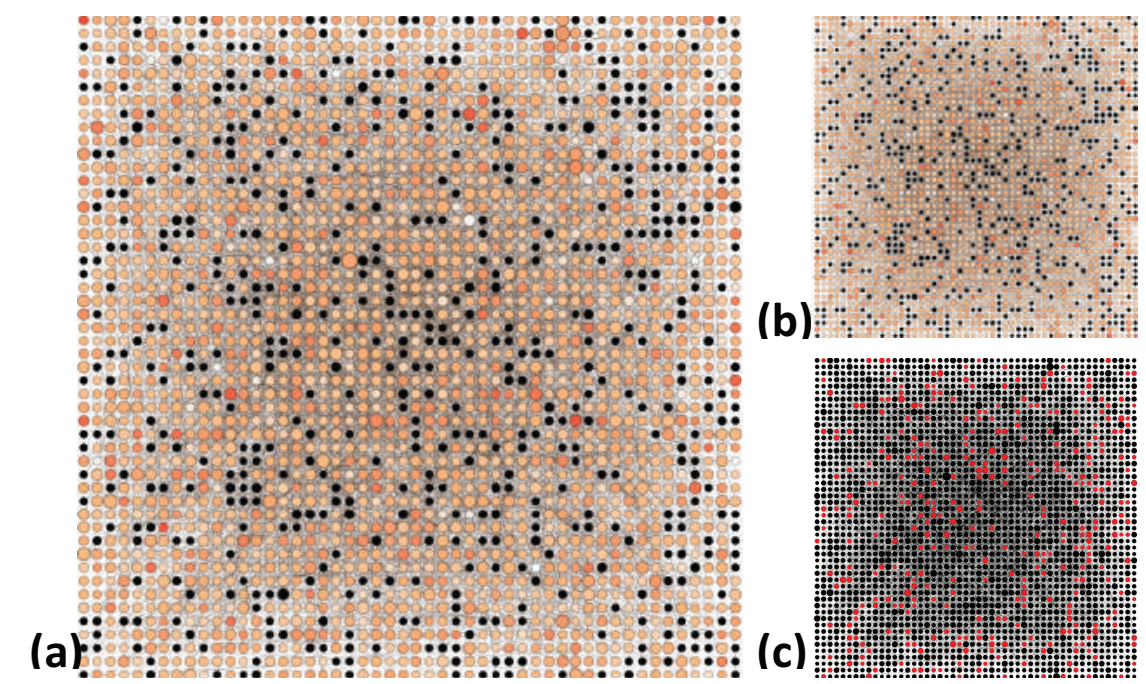

Figure A.5: Heatmap of an Erdos-Renyi network with random force directions. (a) Nodes are influenced by more nodes when both connections and force directions pattern are random. (b) This is true regardless of the position of the node in grid space. (c) There are also more nodes that are not influenced by any node which accounts for $14.12 \%$ of network nodes.

or number of connections have paths to a significant number of nodes in the network as shown in Fig. A.7. This lead to less nodes having no outgoing edge $(68.26 \%$ reduction in nodes with no outgoing edge) which in effect distributes influence in the network.

\section{A.4 Barabasi-Albert Network Configurations}

The fraction of disconnected edges shown in Fig. 6.13(e-f) and the lifetime of grid networks of Barabasi-Albert networks in Fig. 6.15 are averages of two configurations of network hub placement, (1) when the hubs are placed next to each other at the corner of the grid as in Fig. A.8(a, c and e) and (2) when the hub placements are shuffled as in Fig. A.8(b, d and f). Additionally, for the uniform force directions case, both (a) edges directed away from the node with the higher degree as depicted in Fig. A.8( $\mathrm{a}$ and b) and (b) edges directed towards the node with the higher degree as shown in Fig. A.8(c and d) are included in the results presented. 

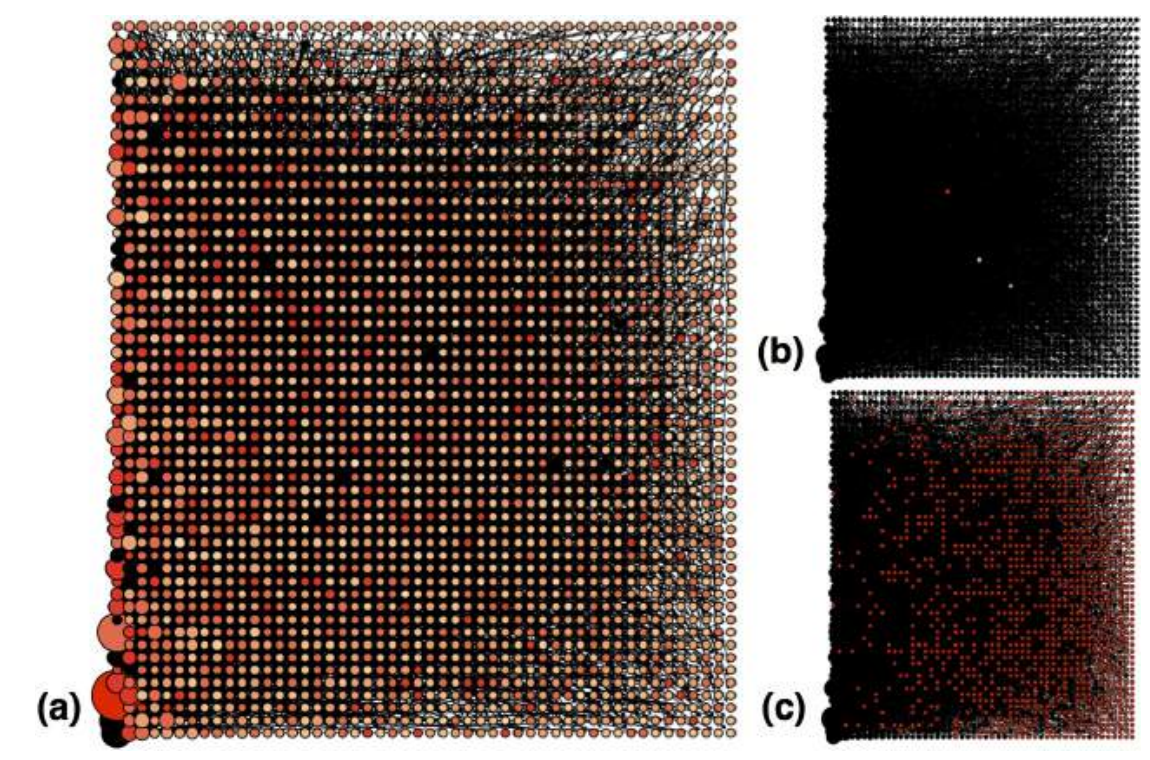

Figure A.6: Heatmap of a Barabasi-Albert network with uniform force directions. (a) The node with the highest degree is connected to almost all nodes. As illustrated in (b), due to the uniformity of force directions and the scale-free degree distribution, other nodes are connected to very few nodes. (c) $49.28 \%$ of nodes in the network has no outgoing edge.

\section{A.5 Generalization to Other Kinds of Spatial Net- works}

Starting with an initial spatial grid network with $N \times M$ nodes located in all intersections made by the $(N-1) \times M+(M-1) \times N$ edges, we can obtain other spatial arrangements by finding a spatial grid network with the least number of intersections such that the actual spatial network can be overlapped unto it in such a way that every node corresponds to one and only one intersection in the initial spatial grid network. That is, we look for $d$ given below which represents the $d \mathrm{x} d$ size of each cell in our initial spatial grid network.

$$
d=\min \left(G C D\left(\left\{\Delta x_{i, i+1}\right\}\right), G C D\left(\left\{\Delta y_{i, i+1}\right\}\right)\right)
$$

where $i=0, \ldots, n-1, n$ is the number of nodes, $\left\{\Delta x_{i, i+1}\right\}$ is the set of horizontal distances between the $i^{\text {th }}$ and $(i+1)^{t h}$ node, and $\left\{\Delta y_{i, i+1}\right\}$ is the set of vertical 


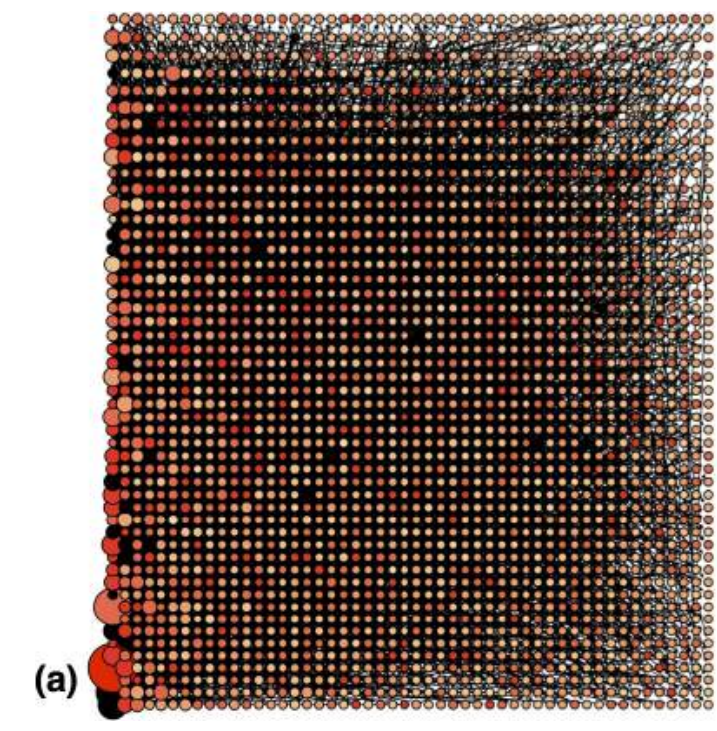

(b)

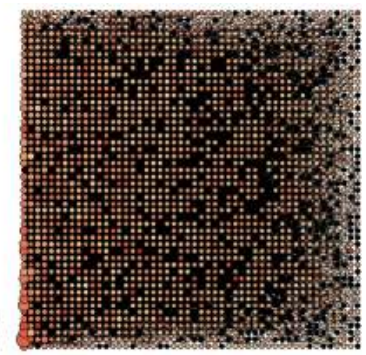

(c)

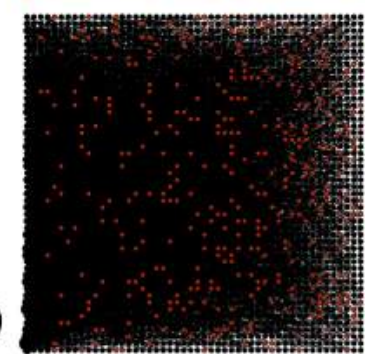

Figure A.7: Heatmap of a Barabasi-Albert network with random force directions. (a) The node with the highest degree is connected to almost all nodes. (b) Randomizing force directions allowed other nodes to have paths to more nodes. (c) $15.64 \%$ of nodes in the network has no outgoing edge.

distances between the $i^{\text {th }}$ and $(i+1)^{t h}$ node such that

$$
\begin{aligned}
& \Delta x_{i, i+1} \% d=0 \\
& \Delta y_{i, i+1} \% d=0 .
\end{aligned}
$$

We retain the nodes of the initial spatial grid network that corresponds to a node in the actual spatial network and remove those that does not correspond to any node in the actual spatial network. Hence, although most real world networks are not arranged spatially in a grid, we can extend the model developed for spatial grid networks to other networks with a different kind of spatial arrangement by systematically removing nodes to mimic real world networks we want to apply the model to.

\section{A.6 Cluster Sizes}

To further explore which among the networks considered is the most resilient, we show the fragmentation and evolution of cluster sizes in lattice, Erdos-Renyi, and Barabasi-Albert networks for $F_{a p p}=0.6$. From Fig. A.10, we find that although the 

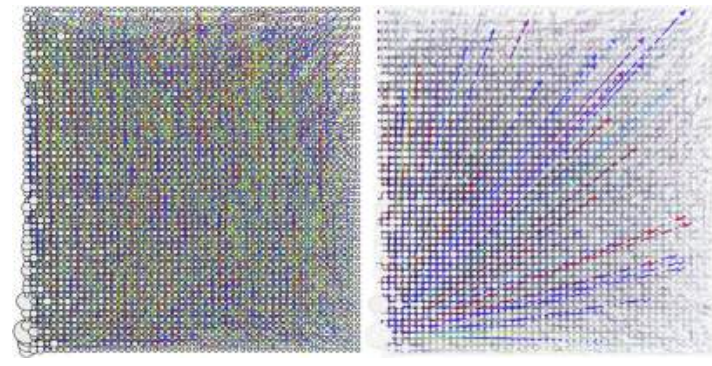

(a) hubs in corner, edges away from higher $k$ node

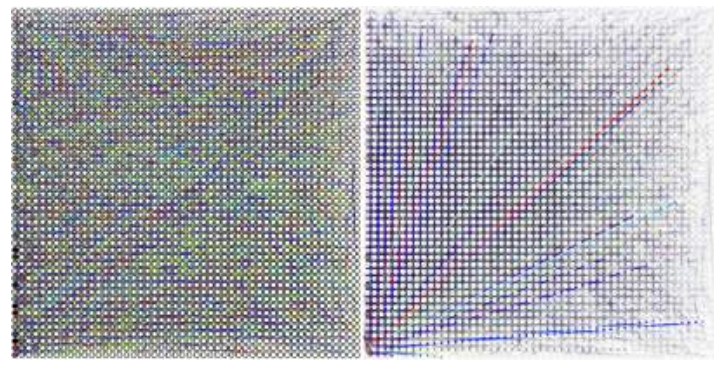

(c) hubs in corner, edges towards higher $k$ node

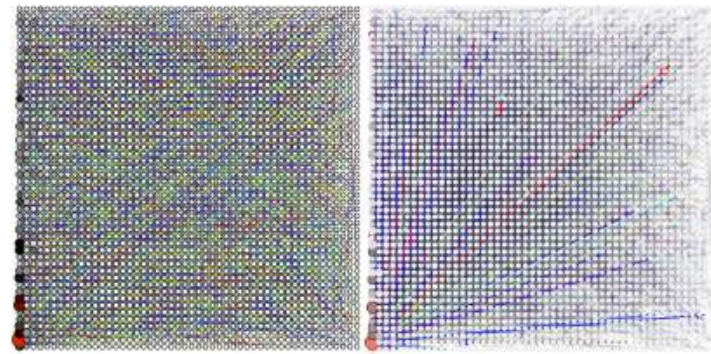

(e) hubs in corner, edges in random directions

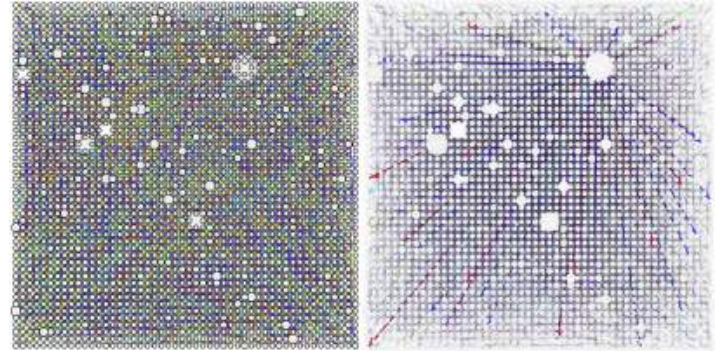

(b) hubs shuffled, edges away from higher $k$ node

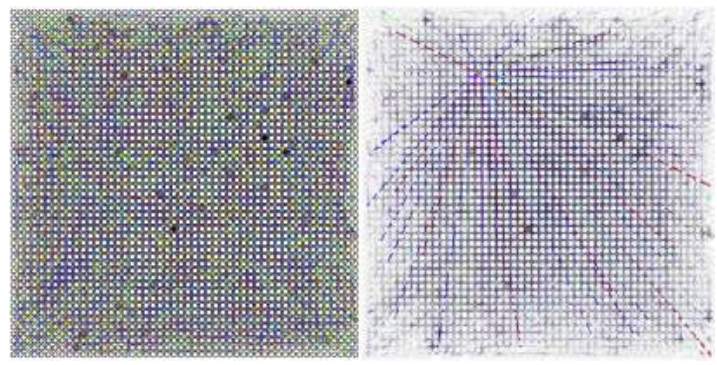

(d) hubs shuffled, edges towards higher $k$ node

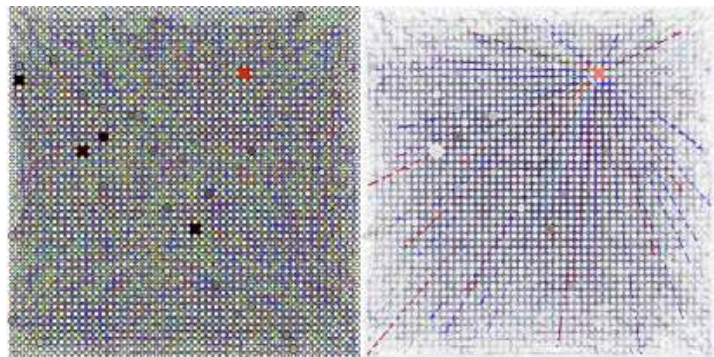

(f) hubs shuffled, edges in random directions

Figure A.8: Barabasi-Albert network configurations. Hubs are either placed next to each other at the corner $(\mathrm{a}, \mathrm{c}, \mathrm{e})$ or in random positions $(\mathrm{b}, \mathrm{d}, \mathrm{f})$. Edges in both placements are directed away from the node with the higher degree $k(\mathrm{a}, \mathrm{b})$, towards the node with the higher degree $k(\mathrm{c}, \mathrm{d})$ or randomly $(\mathrm{e}, \mathrm{f})$. 


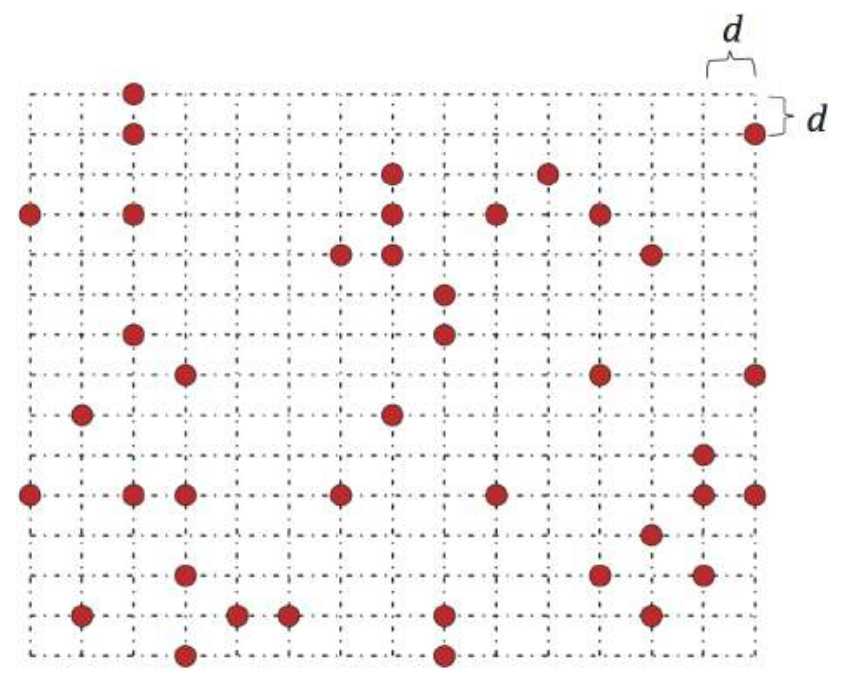

Figure A.9: Spatial Grid. For any spatial arrangement of nodes, a smallest spatial grid can be found such that all nodes lie in one and only one intersection in the grid.

lattice network with random force directions starts to break early in the simulation, it eventually becomes the least fragmented.

Fig. A.11 also shows that when the force sources are positioned randomly in the lattice network, a giant cluster remains intact in the network. However, for the case of positioning the force sources uniformly on the lattice network, the final configuration of the network consists of small clusters with no giant cluster. For small time scales, a lattice network with uniform force directions is more robust. However, longer time scales entail the need to randomize the position of the influential nodes in the lattice network to prevent total network collapse.

The Erdos-Renyi network (Fig. A.12), on the other hand, fragments significantly early on in the evolution of the network. But the subsequent fragmentation of its clusters is much slower than in the lattice network. This initial fast rate of fragmentation of the Erdos-Renyi network can be attributed to its initial structure having several clusters as opposed to the lattice network which starts as one giant cluster.

From Fig. A.10, the fragmentation of the Barabasi-Albert network resembles that of the Erdos-Renyi network. However, examining the size of the largest component (Fig. A.12 and A.13) reveals that the presence of hubs in a Barabasi-Albert network slows down further the fragmentation of the largest cluster in the early stages of fast breakings as compared to an Erdos-Renyi network. 


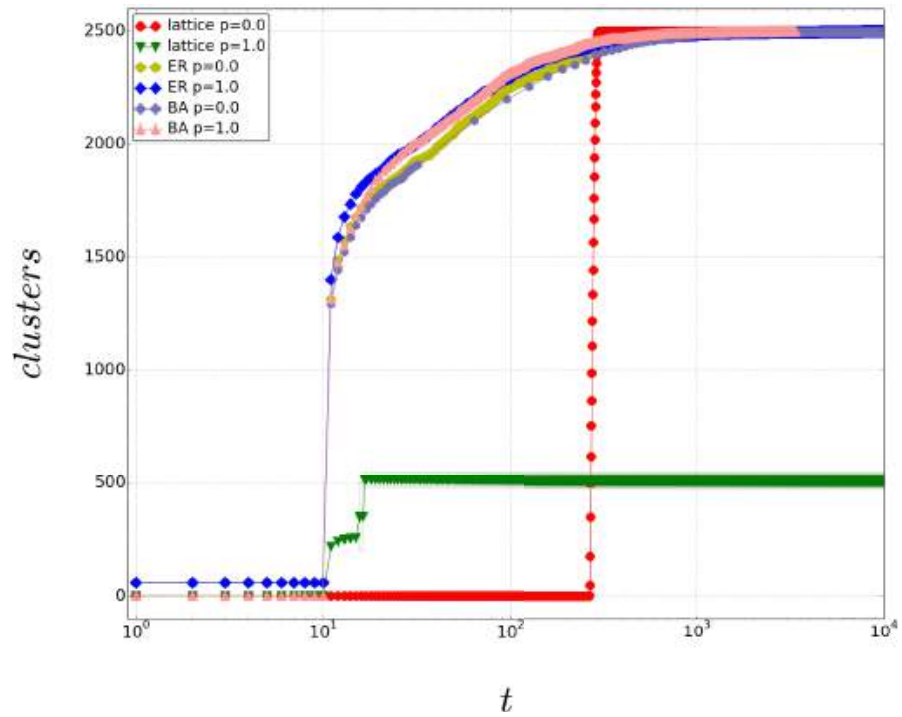

Figure A.10: Number of clusters in the evolving lattice, Erdos-Renyi (ER) and Barabasi-Albert (BA) networks for $F_{a p p}=0.6$. A lattice with random forcing directions experiences the least amount of fragmentation.

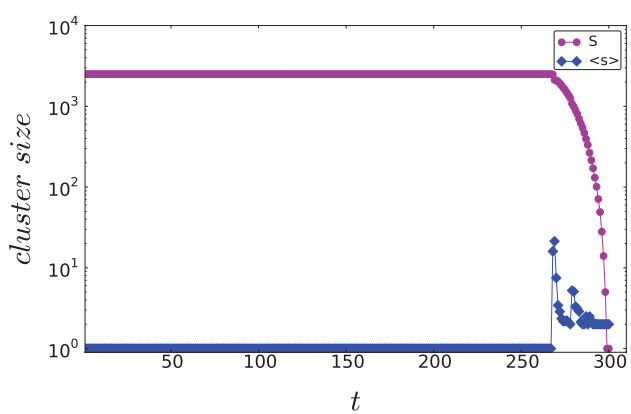

(a)

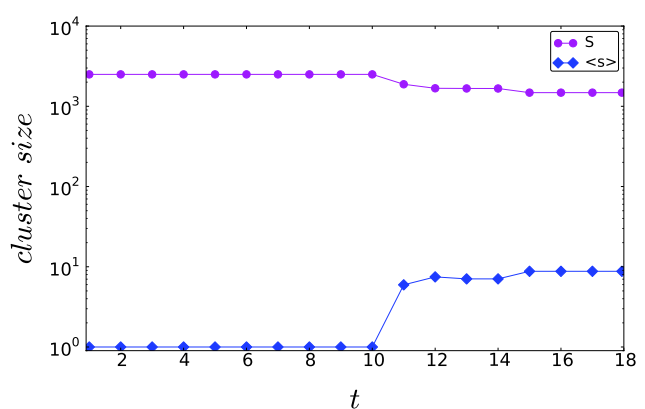

(b)

Figure A.11: Cluster sizes of lattice networks. Size of the largest cluster $S$ and the average size of the smaller clusters $\langle s\rangle$ as the lattice network is subjected to (a) uniform and (b) random force directions with $F_{a p p}=0.6$. Random force directions leads to a percolating cluster while uniformity in force directions causes nodes to detach from each other. 


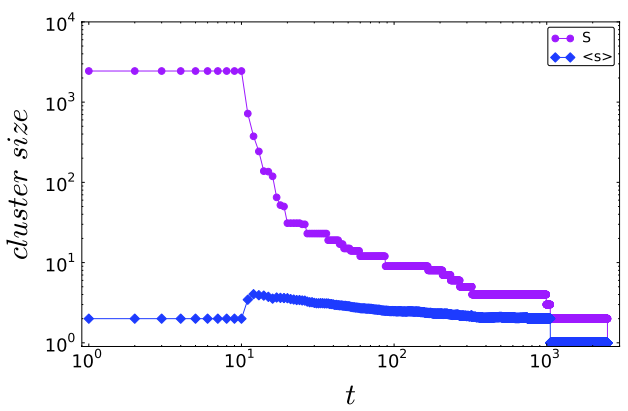

(a)

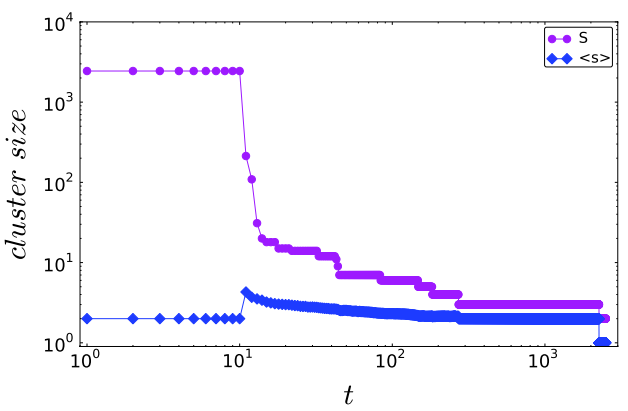

(b)

Figure A.12: Cluster sizes of Erdos-Renyi networks. Size of the largest cluster $S$ and the average size of the smaller clusters $\langle s\rangle$ as the Erdos-Renyi network is subjected to (a) uniform and (b) random force directions with $F_{a p p}=0.6$. The network fragments early on in its evolution leading eventually to isolated nodes.

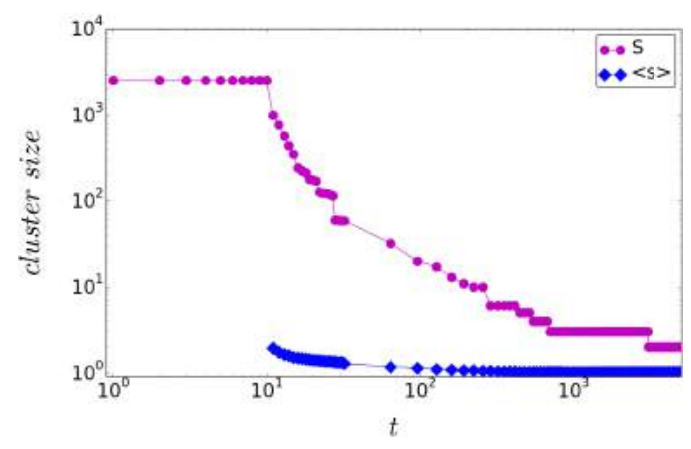

(a)

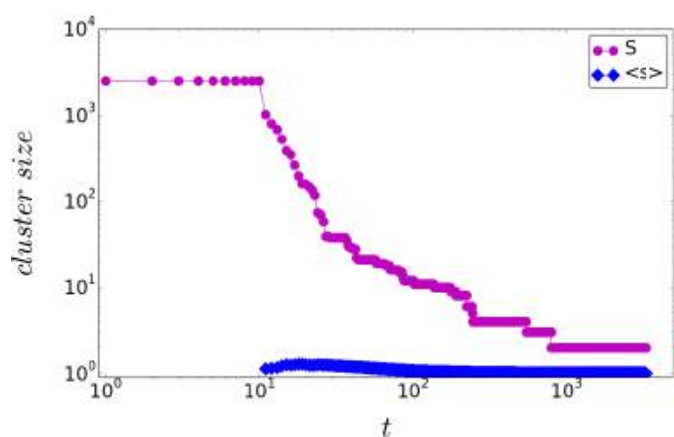

(b)

Figure A.13: Cluster sizes of Barabasi-Albert networks. Size of the largest cluster $S$ and the average size of the smaller clusters $\langle s\rangle$ as the Barabasi-Albert network is subjected to (a) uniform and (b) random force directions with $F_{a p p}=0.6$. 